\title{
THE CALDERÓN PROBLEM FOR CONNECTIONS
}

\author{
Mihajlo Cekić
}

Trinity College

and

Department of Pure Mathematics and Mathematical Statistics

University of Cambridge

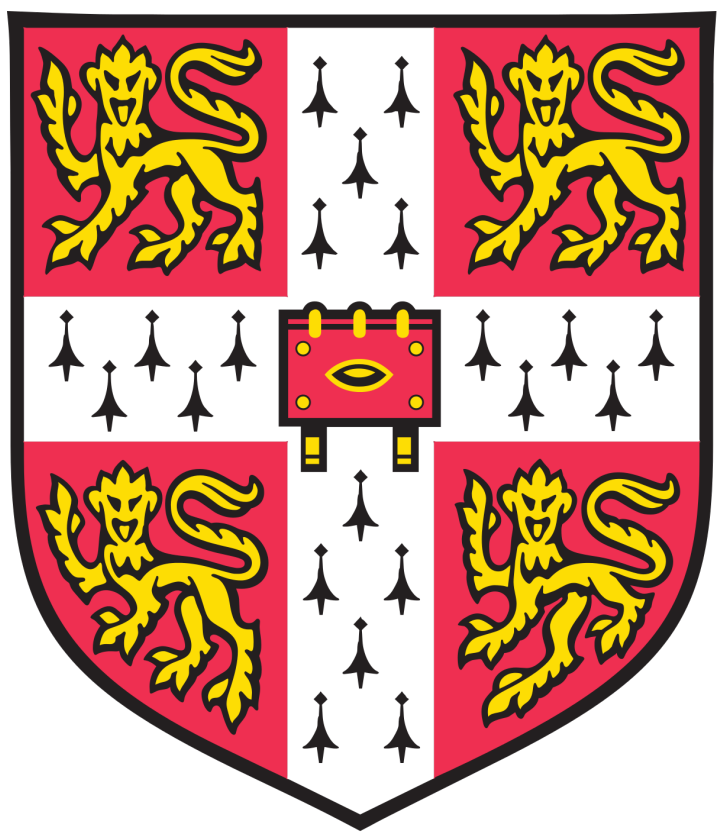

The dissertation is submitted for the degree of Doctor of Philosophy 



\title{
The Calderón problem for connections
}

\author{
Mihajlo Cekić
}

\section{SUMMARY}

This thesis is concerned with the inverse problem of determining a unitary connection $A$ on a Hermitian vector bundle $E$ of rank $m$ over a compact Riemannian manifold $(M, g)$ from the Dirichlet-to-Neumann (DN) map $\Lambda_{A}$ of the associated connection Laplacian $d_{A}^{*} d_{A}$. The connection is to be determined up to a unitary gauge equivalence equal to the identity at the boundary.

In our first approach to the problem, we restrict our attention to conformally transversally anisotropic (cylindrical) manifolds $M \Subset \mathbb{R} \times M_{0}$. Our strategy can be described as follows: we construct the special Complex Geometric Optics solutions oscillating in the vertical direction, that concentrate near geodesics and use their density in an integral identity to reduce the problem to a suitable $X$-ray transform on $M_{0}$. The construction is based on our proof of existence of Gaussian Beams on $M_{0}$, which are a family of smooth approximate solutions to $d_{A}^{*} d_{A} u=0$ depending on a parameter $\tau \in \mathbb{R}$, bounded in $L^{2}$ norm and concentrating in measure along geodesics when $\tau \rightarrow \infty$, whereas the small remainder (that makes the solution exact) can be shown to exist by using suitable Carleman estimates.

In the case $m=1$, we prove the recovery of the connection given the injectivity of the $X$-ray transform on 0 and 1-forms on $M_{0}$. For $m>1$ and $M_{0}$ simple we reduce the problem to a certain two dimensional new non-abelian ray transform.

In our second approach, we assume that the connection $A$ is a Yang-Mills connection and no additional assumption on $M$. We construct a global gauge for $A$ (possibly singular at some points) that ties well with the DN map and in which the Yang-Mills equations become elliptic. By using the unique continuation property for elliptic systems and the fact that the singular set is suitably small, we are able to propagate the gauges globally. For the case $m=1$ we are able to reconstruct the connection, whereas for $m>1$ we are forced to make the technical assumption that $(M, g)$ is analytic in order to prove the recovery.

Finally, in both approaches we are using the vital fact that is proved in this work: $\Lambda_{A}$ is a pseudodifferential operator of order 1 acting on sections of $\left.E\right|_{\partial M}$, whose full symbol determines the full Taylor expansion of $A$ at the boundary. 



\section{Declaration}

This dissertation is the result of my own work and includes nothing which is the outcome of work done in collaboration except as declared in the Preface and specified in the text.

It is not substantially the same as any that I have submitted, or, is being concurrently submitted for a degree or diploma or other qualification at the University of Cambridge or any other University or similar institution except as declared in the Preface and specified in the text. I further state that no substantial part of my dissertation has already been submitted, or, is being concurrently submitted for any such degree, diploma or other qualification at the University of Cambridge or any other University or similar institution except as declared in the Preface and specified in the text.

Mihajlo Cekić 31 July, 2017. 



\section{Acknowledgements}

All this work would not be possible without the support and guidance of my mentor, Professor Gabriel Paternain. Furthermore, I am indebted for the love and care of my parents and my girlfriend Danica Kosanović, throughout the years of my doctoral studies. I am also grateful to Trinity College for financial support.

I would also like to acknowledge the help of Colin Guillarmou, Rafe Mazzeo, Mikko Salo and Gunther Uhlmann, for useful conversations and answering long emails. 



\section{Contents}

Chapter 1. Introduction 1

1.1. Notation 1

1.2. Overview of the main problem 2

$\begin{array}{lll}1.3 . & \text { The CGO approach } & 6\end{array}$

\begin{tabular}{lll}
\hline 1.4. & The Yang-Mills case & 9
\end{tabular}

Chapter 2. Preliminaries 13

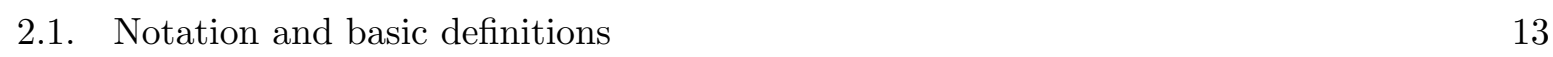

$\begin{array}{lll}2.2 . & \text { The integral identity } & 18\end{array}$

2.3. Semiclassical pseudodifferential operators on manifolds 20

\begin{tabular}{lll}
\hline Chapter 3. & Boundary determination for a connection and a matrix potential & 27
\end{tabular}

\begin{tabular}{ll}
3.1. & PDOs on vector bundles \\
\hline
\end{tabular}

$\begin{array}{lll}3.2 . & \text { Boundary determination } & 28\end{array}$

Chapter 4. Gaussian Beams 33

4.1. Main constructions of Gaussian Beams 33

4.2. Application of Gaussian Beams 44

\begin{tabular}{lll}
\hline Chapter 5. & Carleman estimates & 47
\end{tabular}

Chapter 6. The non-abelian ray transform reduction and simple manifolds 55

6.1 . The CGO construction for simple transversal manifolds 55

6.2. Consequences of the CGO construction and recovering the connection 58

\begin{tabular}{lll}
\hline Chapter 7. & Proof of the Main Theorem I & 65
\end{tabular}

\begin{tabular}{lll}
\hline 7.1. & The main recovery & 65
\end{tabular}

\begin{tabular}{lll}
\hline 7.2 . The holonomy argument & 74
\end{tabular}

\begin{tabular}{lll}
\hline Chapter 8. & Proof of the Main Theorem II & 79
\end{tabular}

$\begin{array}{lll}8.1 . & \text { Recovering a Yang-Mills connection } & 79\end{array}$

8.2. Recovering a Yang-Mills connection for $m>1$

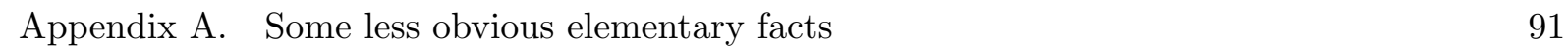

\begin{tabular}{ll}
\hline A.1. The DN map & 91
\end{tabular}

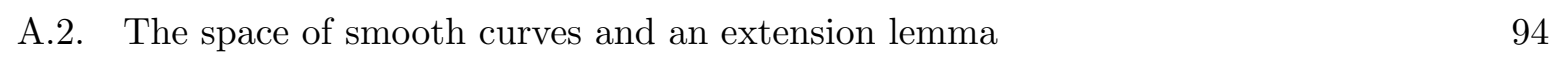

\begin{tabular}{lr}
\hline Bibliography & 97
\end{tabular} 



\section{CHAPTER 1}

\section{Introduction}

In a physical situation with a geometric context, that is modelled by a system of partial differential equations, geometric inverse problems are concerned with the reconstruction of parameters governing this system from the measured data. Many of such physical problems are very conveniently formulated on manifolds, using geometric concepts of metrics, connections, geodesics etc. For the sake of completeness, let us list a few such notable problems:

- The Calderón problem: reconstruct the metric from the Dirichlet-to-Neumann (DN) map (see 84 for a survey)

- $X$-ray transform problems: recover a function or a tensor from integrals along geodesic: 11 (see 65] for a recent survey)

- The boundary rigidity problem: determine the metric from the boundary distances (see the introductions to $\mathbf{6 7}$ and to the recent $[\mathbf{7 5}$ )

- Inverse problems in spectral geometry (see $\mathbf{1 5}$ for a survey)

Each of these problems is a theoretical generalisation of a real-world problem in physics or engineering; the corresponding applications are, respectively, in the Electric Impedance Tomography (EIT), computerised tomography and medical imaging, seismic imaging, identifying distant objects in the universe.

The full Calderón problem consists in determining a metric $g$ on a manifold up to an isometry that fixes every point of the boundary from the DN map. It has been one of the main drives in the area of geometric inverse problems. In this generality the problem is still open, but considerable partial results exist under suitable assumptions on the manifold. Moreover, a very interesting variation is the "twisted" version (with a connection), where we consider the connection Laplacian $\mathcal{L}=\nabla^{*} \nabla$, with $\nabla$ a covariant derivative and $\nabla^{*}$ its formal adjoint. By identifying the components of a connection over the trivial line bundle on the space $\mathbb{R}^{3}$ with a magnetic potential, one gets this operator to be the magnetic Schrödinger operator. In this case, the obstruction to injectivity is the group of gauge transformations that fix the boundary, but the quantity that is preserved is the magnetic field, that is given by the curvature (first Chern class).

In what follows we will briefly survey the results and provide some motivation for the problems in the thesis, which is concerned with the Calderón problem for connections (or the inverse problem for the magnetic Schrödinger equation) and give an overview of the existing results.

\subsection{Notation}

We write $f \lesssim g$, if there exists a constant $C>0$ such that $\|f\| \leq C\|g\|$ for an appropriate norm $\|\cdot\|$.

We write $d V_{g}, d \omega_{g}$ or just $d V$ for the volume form on the Riemannian manifold $(M, g)$.

\footnotetext{
$\overline{1_{\text {This problem }}}$ was first considered in the well-known paper by Radon $\sqrt[68]{ }$ in 1917, where he proved a reconstruction formula - it is interesting to note that it took more than fifty years to find an application for this problem in computerised tomography.
} 
For a domain $\Omega \subset \mathbb{R}^{n}$, we will write $C_{0}^{\infty}(\Omega)=C_{c}^{\infty}(\Omega)$ for the space of compactly supported smooth functions in $\Omega$. For a manifold with boundary $M$, we will write $C_{0}^{\infty}(M)=C_{c}^{\infty}(M)$ to denote the space of smooth functions, compactly supported in the interior $M^{i n t}=M^{\circ}$ of $M$.

By $W^{k, p}(X)$ we will denote the $L^{p}$ based Sobolev space with $k$ weak derivatives; the $L^{2}$ based space will be denote by $H^{k}(X)=W^{k, 2}(X)$. Here $X$ is either a manifold or a subdomain of $\mathbb{R}^{n}$.

For clarity, let us list some of the abbreviations frequently used in the text: CGO for the Complex Geometrical Optics, CTA for the Conformally Transversally Anisotropic, DN for the Dirichlet-to-Neumann map, LCW for Limiting Carleman Weights, UCP for the Unique Continuation Principle and SUCP for the Strong Unique Continuation Principle, YM for YangMills, DCT for the Dominated Convergence Theorem.

By $C^{\infty}(M ; E)$ we denote the space of smooth sections of a vector bundle $E$ over a manifold $M$.

For topological spaces $X$ and $Y$ with $X \subset Y$, we write $X \Subset Y$ to say that the closure of $X$ in $Y$ is compact.

\subsection{Overview of the main problem}

We will now set out a bare minimum of notation to state the main questions in this thesis. Let $(M, g)$ be a compact Riemannian manifold of dimension $n$ with non-empty boundary, $E$ a Hermitian vector bundle of rank $m$ over $M$ equipped with a unitary connection $A$. We will identify the connection $A$ with the associated covariant derivative that we will denote by $d_{A}=d+A$ where $A$ is now a matrix of one-forms, by slightly abusing the notation. Given this, we may define the associated DN map $\Lambda_{A}: C^{\infty}\left(\partial M ;\left.E\right|_{\partial M}\right) \rightarrow C^{\infty}\left(\partial M ;\left.E\right|_{\partial M}\right)$ by uniquely solving the Dirichlet problem:

$$
d_{A}^{*} d_{A}(u)=0,\left.\quad u\right|_{\partial M}=f
$$

and setting $\Lambda_{A}(f)=d_{A}(u)(\nu)$, where $\nu$ is the outwards pointing normal at the boundary. Furthermore, if given a section $Q$ of the endomorphism bundle of $E$, also called an electric potential, then one can solve the associated problem for the operator $\mathcal{L}_{A, Q}:=d_{A}^{*} d_{A}+Q$ and denote the corresponding DN map by $\Lambda_{A, Q}$.

A gauge equivalence $\psi$ is a section of the automorphism bundle Aut $E$, that is a bundle isomorphism that preserves the Hermitian structure. One then has a natural gauge invariance of $\Lambda_{A}$ (coordinate change invariance) - if we denote the pullback connection by $d_{B}=\psi^{*} d_{A}=$ $\psi^{-1} d_{A} \psi$ and in addition we assume $\left.\psi\right|_{\partial M}=I d$, then $\Lambda_{A}=\Lambda_{B}$. As with many similar inverse problems, the question is: is this the only obstruction to injectivity of the map $A \mapsto \Lambda_{A}$ ? One can then pose the following question, which is the main protagonist of the thesis $\mathbf{2}, \mathbf{1 1}, \mathbf{2 1}, \mathbf{2 2}$, $26,45,60,71,77$ :

Conjecture A (Uniqueness). Given two unitary connections $A$ and $B$ on $E$, we have the equivalence: $\Lambda_{A}=\Lambda_{B}$ if and only if there exists a gauge equivalence that is the identity at the boundary that pulls back $B$ to $A$.

If we add a potential $Q$, then $\Lambda_{A, Q}$ is invariant under the change $(A, Q) \mapsto\left(\psi^{*} A, \psi^{*} Q\right)$, where $\psi^{*} Q=\psi^{-1} Q \psi$ and we have the corresponding conjecture. Let us note here that the invariance present in this problem is analogous to the Calderón problem for metrics, where the DN map $\Lambda_{g}$ is defined by solving the Dirichlet problem for the metric Laplacian $\Delta_{g}$. As before, we have an invariance under coordinate change, i.e. $\Lambda_{g_{1}}=\Lambda_{g_{2}}$ if $g_{2}=\varphi^{*} g_{1}$, where $\varphi: M \rightarrow M$ is a diffeomorphism and $\left.\varphi\right|_{\partial M}=I d$ - the question is whether this is the only obstruction to 
injectivity of $g \mapsto \Lambda_{g}$. The geometric approach to these problems has the advantage that the presence of a coordinate change symmetry is most naturally understood.

Besides uniqueness, there are a few other topics that have been studied and that belong to the class of common questions one can ask about inverse problems - they are usually ill-posed, failing uniqueness and have bad stability properties. Here is such a list of possible problems [71]:

- Uniqueness: as posed above in Conjecture A

- Boundary determination: given $\Lambda_{A, Q}$, determine the values of $A$ and $Q$ at the boundary $\partial M$ (and the normal derivatives).

- Partial data: if the measurements are available only on a part of the boundary, i.e. $\left.\Lambda_{A, Q}\right|_{\Gamma}=\left.\Lambda_{B, Q^{\prime}}\right|_{\Gamma}$ for $\Gamma \subset \partial M$ open, prove $F^{*}(B)=A$ and $F^{*}\left(Q^{\prime}\right)=Q$ for some $F$ that fixes $\left.E\right|_{\Gamma}$.

- Reconstruction: given $\Lambda_{A, Q}$ and $E$ trivial line bundle, find an algorithm to reconstruct $d A$ and $Q$.

- Stability: assuming $\Lambda_{A, Q}$ and $\Lambda_{B, Q^{\prime}}$ are close, prove $Q$ and $Q^{\prime}$, and $A$ and $B$ are close in some sense.

Let us now survey some partial answers to the Conjecture $\mathrm{A}$ and revise the main ideas present in the proofs. Firstly, there is a big distinction between the $n=2$ and $n \geq 3$ case. In the $n=2$ case, the conjecture has been fully solved (including a potential and low regularity) by Albin, Guillarmou, Tzou and Uhlmann 2 (see also [36]). Their method is based on a reduction argument to a first order Dirac system on an auxiliary bundle, on which they construct the Complex Geometrical Optics (CGO) solutions using a "Morse holomorphic phase"; this is all special, since such a reduction is only valid for surfaces, where $\Delta_{g}$ factors in a nice way. The partial data case for trivial line bundles was covered relatively recently by Tzou [82, by using a geometric reflection method; the same author and Andersson consider the stability problem in the same setting $[\mathbf{3}]$.

In the latter $n \geq 3$ case, the full question is still open. In general, the metric and connection Calderón problems have a lot of things in common (as we will see) and historically, progress in the metric problem was followed by the analogous progress in the connection problem $\mathbf{1 0}$. One of the essential methods is due to Sylvester and Uhlmann $[\mathbf{7 8}$, who prove the identifiability in the metric problem for conformally Euclidean metric in domains and can be summarised in the following few steps:

(1) Prove a suitable integral identity based on integration by parts.

(2) Prove the necessary Carleman estimates and obtain the existence of the Complex Geometrical Optics (CGO) solutions.

(3) Insert these solutions in the identity and use their density to make a global conclusion about the involved quantities.

(4) Reduce the problem to a question of injectivity of an X-ray transform (or some other transform).

Complex Geometric Optics (CGO) solutions are the solutions of $\mathcal{L}_{A, Q} u=0$, of the form $u=e^{-\frac{\varphi}{h}}\left(a+r_{h}\right)$, where we think of $a$ as an approximate solution

$$
e^{\frac{\varphi}{h}} \mathcal{L}_{A, Q} e^{-\frac{\varphi}{h}} a \approx 0
$$

and $r_{h}$ is a small residue that makes this solution precise, solving an inhomogeneous equation. Furthermore, $h>0$ is a small parameter which we will take in the limit to zero and $\varphi$ is a suitable Carleman weight. The CGO solutions were first constructed for the conductivity equation in 
$\mathbb{R}^{n}$ in $\left[\mathbf{7 8}\right.$ (equivalently solving $\Delta_{c e} u=0$, where $e$ Euclidean metric and $c$ a scalar function), motivated by Calderón's original exponential solutions.

1.2.1. Euclidean case. Based on the steps (1)-(4), there has been a number of partial results for the conjecture for full data, Euclidean subdomains and trivial line bundles, while gradually reducing the regularity of $A$ and $Q$ : possibly the first one is by Sun [77, where the author considers the $n \geq 3$ case and proves identification of a generic electric $W^{1, \infty}$ potential and a small $W^{2, \infty}$ magnetic field, by adapting the CGO construction. Under a smoothness assumption, the CGOs were constructed for any $A$ by Nakamura and Uhlmann $[\mathbf{5 8},[59]$, who used a pseudodifferential conjugation method (i.e. they perform a pseudodifferential gauge transformation) to reduce to the case of a small $A$, which can then be solved by a Neumann series argument. Moreover, the same authors and Sun $6 \mathbf{6 0}$ prove identification in the smooth case for any $A$ and $q$. Tolmasky, Panchenko and Salo reduce the regularity assumption to $C^{1}$, some less regular, but small potentials and Dini continuous, respectively, in the upcoming years in $[\mathbf{7 9},[\mathbf{6 3}$ and $\mathbf{7 2}$. Arguably the best results were obtained by Krupchyk and Uhlmann [45] who reduce the regularity assumption to only $L^{\infty}$ electric and magnetic potentials, while Haberman reduces the regularity to small $A \in W^{s, 3}$ and $q \in W^{-1,3}$ in $\mathbb{R}^{3}$ for $s>0$. In [81], Tzou quantifies the uniqueness results and proves a "log log" stability estimate for partial data, containing measurements on slightly more than half of the boundary. Finally, a very important result for us, the first one to consider systems ( $m>1$ case) that we are aware of is by Eskin [26], where he proves the identifiability of a $C^{\infty}$ magnetic and electric potential.

1.2.2. CTA manifolds. On manifolds, we will mostly limit ourselves to the geometry of special type of cylindrical manifolds, on which we may construct the CGO solutions:

Definition B. Let $(M, g)$ be a smooth, compact, $n$-dimensional Riemannian manifold with boundary, such that $n \geq 3$ and let $T=\left(\mathbb{R} \times M_{0}, e \oplus g_{0}\right)$, where $e$ is the Euclidean metric and $\left(M_{0}, g_{0}\right)$ a compact $(n-1)$-dimensional Riemannian manifold with boundary. We say that $(M, g)$ is conformally transversally anisotropic $(C T A)$ if $(M, c g)$ is isometrically embedded into $T$ for some positive function $c$ on $M$.

If $M$ is CTA with $M_{0}$ simpl ${ }^{2}$ in $2 \mathbf{2 1}$ the conjecture was reduced to an injectivity problem of the $X$-ray transform on $M_{0}$, which is well known [65]. For the metric problem, in [23] CGOs are constructed for any compact $M_{0}$ and an electric potential is identified if $M_{0}$ has just an injective $X$-ray transform, by using the approximate solutions concentrating on geodesics called Gaussian Beams, as in (1.2).

In this thesis and in [1], by generalising the Gaussian Beam construction to include the case of connections on arbitrary bundles and considering specific partial data, we were able to gauge identify two connections for $m=1$; for the higher rank $m>1$ case, in the same paper we reduced the conjecture to a new non-abelian ray transform. Finally, in the recent works [46] for the CTA case, generalising [11] when $m=1$, the authors gauge identify two continuous connections (and thus reduce the regularity); for $M_{0}$ simple they identify magnetic fields and electric potentials in the $L^{\infty}$ case.

The partial data problem (measurements available only on a subset of the boundary) in the Euclidean subdomains and trivial line bundles was studied by Dos Santos Ferreira, Kenig, Sjöstrand and Uhlmann $\left[\mathbf{2 2}\right.$, who prove the recovery of a $C^{2}$ magnetic field and $L^{\infty}$ electric

\footnotetext{
${ }^{2} \mathrm{~A}$ Riemannian manifold $(M, g)$ is called simple if the exponential map $\exp _{p}$ is a diffeomorphism onto $M$ from its domain of definition for all points $p$; in addition, one also asks that the boundary is strictly convex (second fundamental form positive definite).
} 
fields, given data on specific subsets of the boundary determined by the acute (or obtuse) angle of rays hitting the boundary from a fixed point in the exterior of the subdomain (front and back faces). Their work is based on the construction of the CGOs for the $A=0$ case that was covered by Kenig, Sjöstrand and Uhlmann [40], who identify two potentials - this was the first instance that the technique of the Carleman estimates was used to construct CGOs. More precisely, the authors introduce the notion of limiting Carleman weights (LCW) and use a convexification idea to prove the estimates. This was extended to Hölder continuous coefficients by Knudsen and Salo [41].

In general, Carleman estimates can be used to prove uniqueness results by using an "algebraic" invariance of the form: $P u=0$ if and only if $P_{\varphi} \omega=0$, where $P_{\varphi}=e^{-\varphi} P e^{\varphi}$ and $\omega=e^{-\varphi} u$, with the aim to obtain positivity in the operator. Here, $\varphi$ is called a Carleman weight and needs to be specified.

Following on the work of [40, Dos Santos Ferreira, Kenig, Salo and Uhlmann 21] have classified all possible LCWs in the Euclidean case and found a nice geometric condition on $(M, g)$ equivalent to having an LCW: the conformal class of $g$ needs to have a unit parallel vector field (see Definition B]; this condition is of course non-generic (see [51]) and it remains an open problem to construct such solutions in full generality. In [13, Chung constructs CGOs for the magnetic equation that have compact support at the boundary and generalises the results of [40] to non-zero $A$. A slightly related to the systems case is the result in [14], where the authors consider the case of differential forms.

1.2.3. Boundary determination. Finally, let us review a few results about boundary determination (see $\mathbf{7 1}$ for more details). The first result that considered the anisotropic case, or equivalently any Riemannian metric, was by Lee and Uhlmann [50, who proved that the full symbol of $\Lambda_{g}$ as a pseudodifferential operator of first order determines the Taylor expansion of $g$ at the boundary - the same method can be applied to other inverse boundary problems [58]. In the Euclidean setting and the case of a trivial line bundle, Nakamura, Sun and Uhlmann prove the boundary determination for any $A$ in $[\mathbf{6 0}]$. However, pseudodifferential methods are limited to the $C^{\infty}$ setting and they assume the well-posedness of the Dirichlet problem, i.e. that zero is not an eigenvalue of $\mathcal{L}_{A, Q}$. There are other methods one can use to prove the identification at the boundary - by testing against oscillatory functions, coefficients at the boundary were retrieved in 9,72 for continuous connections and $L^{\infty}$ potentials; the manifolds case is considered in the recent work of 46 .

Finally, in $\mathbf{1 2}$ we considered the former approach and proved boundary determination in the higher rank $m>1$ case.

1.2.4. Alternative approaches. Another related method for the metric Calderón problem that does not use CGO solutions, in the analytic category, is the one of Lassas and Uhlmann [49], who prove the uniqueness with partial data in their work. Together with Taylor [48], the authors extend their results to non-compact complete Riemannian manifolds with compact boundary. They prove the required result by embedding the manifold in a suitable Sobolev space, by using Green's functions with respect to the metrics and prove the obtained composition is an isometry, by using boundary determination and analytic continuation. A very close approach is by Guillarmou and Sá Barretto who prove the uniqueness for Einstein metrics, by using the unique continuation property with boundary determination for reconstruction near the boundary in harmonic coordinates and then use the Lassas-Uhlmann method. 
Inspired by these results, in [12 we proved uniqueness of the main conjecture for two YangMills connections using an entirely new method.

Finally, we should mention there is a relationship between our inverse boundary value problem and the inverse scattering problem at fixed energy - for compactly supported potentials these two problems are equivalent $[\mathbf{2 8}$ and the scattering problem was studied by different authors in the past 27, 62. However, we will not discuss this approach any further.

1.2.5. The $\mathrm{X}$-ray transform. It is now clear that the injectivity questions of the $X$-ray transforms are one of the central problems in the area of geometric inverse problems. Moreover, it is a fact that many of the mentioned problems and inverse problems in general can be reduced to instances of the $X$-ray transform. This has come up in a few places: in the elliptic inverse boundary value problems in which the CGO solutions are constructed by using the Gaussian Beams - they concentrate along geodesics in an asymptotic limit, yielding integrals of the quantities as in Step (4) 11, 21, 23. Other applications include [66, where the authors determine a matrix $X$-ray transform from the scattering relation and thus reduce the problem. There are many other occasions where a geometric inverse problem is reduced to another one - one such important example is the proof of boundary rigidity for surfaces by Pestov and Uhlmann [67, the main part of which determines the DN map data for the metric from the boundary distances.

Let us now state the main results of this thesis, propose motivation and outline ideas of proof for them: one by constructing the CGO solutions on CTA manifolds for non-zero $A$ and reducing the problem to the injectivity of a ray transform and the other, valid for general manifolds, but special Yang-Mills connections.

\subsection{The CGO approach}

In this section, we describe our approach to Conjecture A in more detail, based on the CGO solutions. Our work completely covers and proves the conjecture for admissible line bundles, in the case of CTA manifolds and with a suitable hypothesis of injectivity of the ray transform on the transversal manifold $M_{0}$ (see Theorem E) - this result is new in the sense that we have significantly weakened the simplicity hypothesis on $M_{0}$, generalising results in [21].

In order to state the Main Theorem, we need to set up some notation, still assuming the background from the previous section: let $F(-\infty)=F=\left\{x \in \partial M \mid\left\langle\frac{\partial}{\partial x_{1}}, \nu(x)\right\rangle=c(x) \nu_{1}(x) \leq\right.$ $0\}$, which we call the front side and the analogous set $B$ with $\leq$ replaced with $\geq$ we call the back side; here $\nu(x)$ is the outer normal. We also use the notation $\partial M_{-}=F$ and $\partial M_{+}=B$ (see Figure 11. Moreover, we remark that this setting was used in 22 in order to prove a suitable partial data result in Euclidean domains; the analogy with our case is that we are considering rays from the "point at infinity", rather than from the points near the boundary.

Furthermore, lets us spell out some basic definitions about the $X$-ray transform. Let $S M_{0}=$ $\left\{(x, \xi) \in \mid x \in M_{0}\right.$ and $\left.|\xi|=1\right\}$ denote the sphere bundle of $M_{0}$ and consider the set of all inward and outward pointing vectors:

$$
\partial_{ \pm} S M_{0}=\left\{(x, \xi) \in S M_{0} \mid x \in \partial M_{0} \text { and } \pm\langle\xi, \nu(x)\rangle \leq 0\right\}
$$

Then, let us denote by $\gamma_{x, \xi}$ the unique geodesic in $M_{0}$ with $\gamma_{x, \xi}(0)=x$ and $\dot{\gamma}_{x, \xi}(0)=\xi$ for any $(x, \xi) \in T M$; we define the exit time $\tau(x, \xi)$ as the first time when $\gamma_{x, \xi}$ hits the boundary $\partial M_{0}$ (possibly infinite). Then we denote the set of trapped geodesics by:

$$
\Gamma_{+}=\left\{(x, \xi) \in \partial_{+} S M_{0} \mid \tau(x, \xi)=\infty\right\}
$$


With this in mind, we may define the geodesic $X$-ray transform of a smooth 1-form $\alpha$ and a function $f$ on $M_{0}$, for all $(x, \xi) \in \partial_{+} S M_{0} \backslash \Gamma_{+}$:

$$
I(f, \alpha)(x, \xi)=\int_{0}^{\tau(x, \xi)}\left(f\left(\gamma_{x, \xi}(t)\right)+\alpha\left(\gamma_{x, \xi}(t), \dot{\gamma}_{x, \xi}(t)\right)\right) d t
$$

There is an obstruction to injectivity of this transform:

Definition C. We say that the $X$-ray transform is injective on functions and 1-forms if $I(f, \alpha)=0$ implies that $f=0$ and the existence of a smooth function $p$ on $M_{0}$ with $\left.p\right|_{\partial M_{0}}=0$ and $\alpha=d p$.

We will need another definition - this time it is about the "admissible" vector bundles over $M$, which is a necessary topological condition to construct the CGO solutions.

Definition D. Let $M \Subset \mathbb{R} \times M_{0}$ be a CTA manifold. A vector bundle $E$ over $M$ is called admissible if it is isomorphic to a pullback bundle $\pi^{*} E_{0}$, where $E_{0}$ is a vector bundle over $M_{0}$ and $\pi: M \rightarrow M_{0}$ is the projection along the $x_{1}$-direction.

Notice the condition of admissibility of the vector bundle $E$ is a necessary and sufficient condition for the bundle $E$ to have an extension $E^{\prime}$ to $\mathbb{R} \times M_{0}$ such that $\left.E^{\prime}\right|_{M}=E$ (easy exercise). We prove the following result:

Theorem E (Main Theorem). Let $(M, g)$ be a CTA manifold. Let $E$ be an admissible Hermitian line bundle over $M$, equipped with unitary connections $A_{1}$ and $A_{2}$. Assume furthermore the injectivity of the ray transform on functions and 1-forms on $M_{0}$. If $\Gamma$ is a neighbourhood of the front face of $M$, then $\left.\Lambda_{A_{1}}(f)\right|_{\Gamma}=\left.\Lambda_{A_{2}}(f)\right|_{\Gamma} \bigsqcup^{3}$ for all $f \in C^{\infty}\left(\partial M ;\left.E\right|_{\partial M}\right)$ implies the existence of a gauge equivalence that is the identity on $\Gamma$ and which pulls back $A_{2}$ to $A_{1}$.

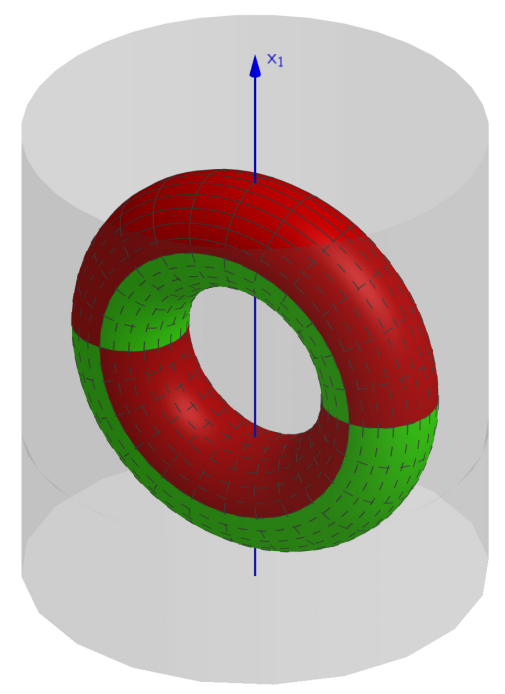

Figure 1. Solid torus as a CTA manifold, showing front (green) and back (red) faces.

Firstly, as mentioned in the overiew, we remark that the CGO solutions supported in a front or a back face were constructed by Chung in $\mathbf{1 3}$ for Euclidean domains - this probably implies such solutions could be constructed in our setting. The existence of such CGOs would reduce

\footnotetext{
${ }^{3}$ Alternatively, given a connection $A$ and a subset $\Gamma \subset \partial M$ of the boundary, the partial Cauchy data space are defined as $C_{A}^{\Gamma}=\left\{\left(\left.u\right|_{\partial M},\left.d_{A} u(\nu)\right|_{\Gamma}\right) \mid d_{A}^{*} d_{A} u=0\right.$ and $\left.u \in H^{1}(M)\right\}$, where $\nu$ is the outward normal; then by definition $C_{A_{1}}^{\Gamma}=C_{A_{2}}^{\Gamma}$ if and only if $\left.\Lambda_{A_{1}}(f)\right|_{\Gamma}=\left.\Lambda_{A_{2}}(f)\right|_{\Gamma}$ for all $f$.
} 
the assumption of the theorem to $\left.\Lambda_{A_{1}}(f)\right|_{\Gamma}=\left.\Lambda_{A_{2}}(f)\right|_{\Gamma}$ for all $f \in C_{0}^{\infty}(\Gamma)$; however, due to technical reasons and simplicity we will deal only with the full Dirichlet data.

This particular CTA setting is interesting because of the existence of the "Euclidean direction" in our manifold, i.e. the direction set out by $\mathbb{R}$; this enables us to define a Carleman weight $\varphi(x)=x_{1}$, which in turn allows for the CGO solutions to be constructed (see [21]; for an alternative construction of the CGOs using the Fourier transform in the $x_{1}$ variable, see [70] $)$. Our construction is based on the solutions known as Gaussian beams, which have already shown to be fertile in the less complicated case of the operator $\Delta+q$ in $[\mathbf{2 3}$. We have also adapted the construction to the case of the connection Laplacian, valid for functions with values in a vector bundle; the idea is to show existence of approximate solutions which concentrate in a suitable way around geodesics. This is done locally in charts covering the geodesic and then glued together to form a global solution. Moreover, it is worth emphasising that our main result Theorem E generalises the one present in [21], in that it does not ask for $M_{0}$ to be simple, which complicates the construction significantly - more concretely, it allows for the geodesics on $M_{0}$ to self-intersect and allows for the existence of conjugate points (which prevent the exponential map from being a diffeomorphism).

Furthermore, in Section 6 another approach based on the interplay between the parallel transport and the unique continuation principle (UCP) for elliptic equations is pursued. Theorem 7.6 proves Conjecture A in the setting of partial data, in the case of two flat connections. The latter assumption simplifies the problem significantly, because the parallel transport along homotopic curves is then the same, which enables us to define a suitable gauge. A similar idea was already used in 36 in the case of line bundles over surfaces. Moreover, there is a natural way of pushing these results further to the case of Yang-Mills connections, which will be considered in the next section.

In addition to the above, we also provide a general framework and base for the future work in the direction of the Calderón problem for connections on vector bundles, by constructing the CGOs in general (see Theorem 4.4 and Remark 4.9). For simple transversal manifolds and the trivial vector bundle of any rank, we also get to the fourth step in our previous analysis - see Section 4. Moreover, in this case, one can reduce the main DN inverse problem to a new non-abelian X-ray transform - see Question F, which we have not found in the literature. The reduction process is fully explained and outlined in Section 6.2. One distinct feature of this transform is that it involves the complex derivative $\mathbb{X}=\frac{\partial}{\partial x_{1}}+i X$, rather than just the usual geodesic vector field derivative $X$ - hence, one could expect that methods from complex analysis and geometry might be useful to deduce certain properties of this transform (as in [26]). Another characteristic property of this transform is that it is not abelian in general, making it harder to reduce to an $X$-ray transform on just $M_{0}$, which is usually done in such situations (see [21]). The question is posed here in the form of a transport equation.

QUESTION F (The non-abelian Radon transform). Let $\left(M_{0}, g_{0}\right)$ be a compact simple manifold with boundary, with $\operatorname{dim} M_{0} \geq 2$ and let $M$ be an isometrically embedded, compact submanifold of $T=\left(\mathbb{R} \times M_{0}, e \oplus g_{0}\right)$ with non-empty boundary and $\operatorname{dim} M=\operatorname{dim} T$. Let $E=\mathbb{R} \times M_{0} \times \mathbb{C}^{m}$ be a Hermitian vector bundle equipped with two unitary connections $A_{1}$ and $A_{2}$, which are compactly supported and satisfy $A_{1}=A_{2}$ on $\mathbb{R} \times M_{0} \backslash M$. Let $R^{\prime}=\left\{\left(x_{1}, x, v\right) \in \mathbb{R} \times S M_{0}:\left(x_{1}, x\right) \notin M\right\}$. Assume we are given a smooth matrix function $G: \mathbb{R} \times S M_{0} \rightarrow G L(m, \mathbb{C})$ such that, if $\mathbb{X}=$ $\frac{\partial}{\partial x_{1}}+i X$, where $X$ is the geodesic vector field:

$$
\mathbb{X} G\left(x_{1}, x, v\right)=-A_{1}\left(x_{1}, x\right)\left(\frac{\partial}{\partial x_{1}}+i v\right) G\left(x_{1}, x, v\right)+G\left(x_{1}, x, v\right) A_{2}\left(x_{1}, x\right)\left(\frac{\partial}{\partial x_{1}}+i v\right)
$$


for all $\left(x_{1}, x, v\right)$, with the additional condition $\left.G\right|_{R^{\prime}}=I d$. Prove that $G$ is independent of the velocity variable $v$.

In order to support our Theorem E, let us list a number of results that generate a large class of non-trivial examples for which our theorem is new. Firstly, the results of Stefanov, Uhlmann and Vasy $\mathbf{7 4}, 85$ give the injectivity of the ray transform if the manifold is foliated by convex hypersurfaces up to a small set; secondly, the result of Guillarmou in $\mathbf{3 3}$ proves the injectivity in the case of manifolds with negative curvature and strictly convex boundary (second fundamental form positive). Finally, the very recent results of Paternain, Salo, Uhlmann and Zhou [66] show that the geodesic transform is injective in the case of strictly convex manifolds with non-negative sectional curvature. The second one of these results allows existence of trapping (geodesics of infinite length), while the third one allows for the existence of conjugate points. As a concrete example of where our Main Theorem is a new result, we can let the transversal manifold $M_{0}$ be a catenoid - a surface with negative curvature and for which the boundary is strictly convex; it has geodesics that are trapped (e.g. the middle circle) and hence is not simple, but the ray transform is injective by the results in $\mathbf{3 3}$.

These results are proved in the thesis as follows: in the next chapter we provide some elementary background and also prove an integral identity based on integration by parts, while Chapters 4 and 5 are the most technical ones - the former one we divide into two parts: in Section 4.1, we present the lengthy construction of the version of Gaussian beam solutions that is relevant for us, for general vector bundles. Furthermore, in Section 4.2 we apply this construction to deduce the existence of CGO solutions and moreover, we prove that we may recover the differential of the connection $d A$ from the DN map in the case of line bundles. In the latter one we prove the necessary Carleman estimates for sections of vector bundles using semiclassical calculus. However, in Chapter 6 we consider the case where the transversal manifold is simple and for which we may construct the ansatz in a much easier way - in this setting, we reduce the conjecture to the new ray transform (here $m>1$ ). Finally, in Chapter 7 we finish the proof of Theorem E: Section 7.1 recovers the magnetic field (curvature) and Section 7.2 concludes the proof by employing an argument involving holonomy and the unique continuation principle.

\subsection{The Yang-Mills case}

In this section, we consider Conjecture $\mathrm{A}$ for a special type of connections, called the YangMills connections. As far as we know, the results in our work $\mathbf{1 2}$ are the first ones that consider the connection problem and do not rely on the CGO solutions (see any of [11,21, 22, 26]), but on unique continuation principles; in this sense, we generalise the result for flat connections from the previous section.

The Yang-Mills connections generalise flat connections and are important in physics and geometry. They are defined by the following equation:

$$
D_{A}^{*} F_{A}=0
$$

where $D_{A}=d_{A}^{\text {End }}$ is the induced connection on the endomorphism bundle End $E$ and $F_{A}$ is the curvature of $A$ (see the overview section for notation). With this in mind, we may formulate the main theorems of this section:

Theorem G. Assume $\operatorname{dim} M \geq 2$, let $E=M \times \mathbb{C}$ be a Hermitian line bundle with standard metric and $\emptyset \neq \Gamma \subset \partial M$ an open, non-empty subset of the boundary. Let $A$ and $B$ be two unitary Yang-Mills connections on $E$. If $\left.\Lambda_{A}(f)\right|_{\Gamma}=\left.\Lambda_{B}(f)\right|_{\Gamma}$ for all $f \in C_{0}^{\infty}\left(\Gamma ;\left.E\right|_{\Gamma}\right)$, then there 
exists a gauge automorphism (unitary) $h$ with $\left.h\right|_{\Gamma}=I d$ such that $h^{*}(A)=B$ on the whole of $M$.

Before further proceeding to the organisation of the proofs, let us explain the source of motivation for considering this problem. The idea came from the analogy between Einstein metrics in Riemannian geometry and Yang-Mills connections on Hermitian vector bundles and also the paper by Guillarmou and Sá Barreto [35]. They prove the recovery of two Einstein manifolds from the DN map for metrics; the method of their proof relies on a reconstruction near the boundary, where in special harmonic coordinates Einstein equations become quasi-linear elliptic (the metric is thus also analytic in the interior in such coordiantes, but not all the way up to the boundary) and hence, by combining the boundary determination result $\mathbf{5 0}$ and a unique continuation result for elliptic systems they prove one can identify the two metrics in a neighbourhood of the boundary. Moreover, by exploiting this analytic structure they observe that the method of Lassas and Uhlmann [48], who prove the analytic Calderón problem for metrics, may be used to extend this local isometry to the whole manifold (this works by embedding the two manifolds in a suitable Sobolev space using the Green functions of the metric Laplacians and the local isometry and showing the appropriate composition is an isometry).

In our case, the analogous concept to harmonic coordinates is to consider the Coulomb gauge 83 which transforms the connection to the one for which $d^{*}(A)=0$, so that the YangMills equations become an elliptic system with principal diagonal part. However, this gauge does not tie well with the DN map and so we must look for something else - Lemma 8.1 gives an answer as to which gauge to consider. In this gauge, we may use a similar unique continuation principle result to yield the equivalence of connections close to the boundary. However, for going further into the interior we designed a new method.

More concretely, our gauge from Lemma 8.1 satisfies the equation $d_{A}^{*} d_{A} F=0$ and so we cannot guarantee that it is non-singular globally. We show that the zero set of the determinant of $F$ is small in the smooth case when $m=1$ and in the analytic case for arbitrary $m$ - it is covered by countably many submanifolds of codimension one, or in the language of geometric analysis it is $(n-1)-C^{\infty}$-rectifiable. Since (the complement of) this singular set can be topologically non-trivial (see Figure 1), we end up with such barriers consisting of singular points of $F$ that prevent us to use the UCP and go inside the manifold. This is addressed by looking at the sufficiently nice points of the barriers and locally near these points, using a degenerate form of UCP (in the smooth case) or a suitable form of analytic continuation (in the analytic case) to extend an appropriate gauge equivalence between the two given connections beyond the barriers; we name this procedure as "drilling". Since we show there is a dense set of such nice points, we may perform the drilling to extend our gauges globally.

Here is what we prove in the analytic case:

Theorem H. Let $(M, g)$ be an analytic Riemannian manifold of dimension $\operatorname{dim} M \geq 2$ and let $\Gamma$ be as in Theorem $G$. If $E=M \times \mathbb{C}^{m}$ is a Hermitian vector bundle with the standard structure and if $A$ and $B$ are two unitary Yang-Mills connections on $E$, then $\left.\Lambda_{A}(f)\right|_{\Gamma}=\Lambda_{B}(f)_{\Gamma}$ for all $f \in C_{0}^{\infty}\left(\Gamma ;\left.E\right|_{\Gamma}\right)$ if and only if there exists a gauge automorphism $H$ of $E$, with $\left.H\right|_{\Gamma}=I d$, such that $H^{*}(A)=B$.

The proof of this theorem also relies on using the Coulomb gauge locally, since the gauge from Lemma 8.1 does not work near singular points; in this gauge we may get that $A$ is analytic and hence $F$ also, since they satisfy elliptic equations with analytic coefficients.

\footnotetext{
${ }^{4}$ The metric $g$ is only assumed to be analytic in the interior of $M$.
} 
Furthermore, the main difficulty for the smooth, higher rank $(m>1)$ case is to prove the strong unique continuation property for the determinant $\operatorname{det} F$ of a solution to $d_{A}^{*} d_{A} F=0$; for $m=1$, this is obvious by standard results. Another issue is that one needs to prove the UCP for elliptic systems with diagonal principal part and higher orders of degeneracy at a hyperplane. More precisely, operators with leading term $x_{n}^{2 k} \Delta \times I d$ and with first order terms containing multiples of $x_{n}^{k}$; in other words, the algebra of operators generated by derivatives of the form $x_{n}^{k} \frac{\partial}{\partial x}$ where $x_{n}$ is the boundary defining coordinate.

In this thesis, an important role is played by the unique continuation results. As a source for the UCP results we will use Bär $[\mathbf{7}$, who proves the rectifiability statements for the zero sets of first order semilinear elliptic systems; for convenience, we prove an easy consequence of his results for second order systems in Lemma 8.4. Furthermore, we apply the degenerate UCP result of Mazzeo [53], for which we have not found an alternative source in the literature.

We also prove that the DN map $\Lambda_{A}$ is an elliptic pseudodifferential operator of order 1 on the restriction of the vector bundle to the boundary and deduce that its full symbol determines the full Taylor series of the connection, metric and a potential at the boundary. This was first proved in the case of a Riemannian metric by Lee and Uhlmann [50 and later considered in the $m=1$ case with a connection in $2 \mathbf{2 1}$; see also the overview section. In Chapter 3 , we generalise this approach to the case of systems and prove the analogous result.

The proofs are organised as follows: in the next chapter, we recall some formulas from differential geometry and make a few observations about choosing appropriate gauges. In Chapter 8 we prove Theorems $\mathrm{G}$ and $\mathrm{H}$; in Section 8.1 we consider the smooth case and prove the global result for $m=1$. Along the way, we construct the new gauge and deduce the UCP result we need. In Section 8.2 we consider the $m>1$ case for analytic metrics, by adapting the proof of the line bundle case and exploiting real-analyticity. As mentioned previously, in Chapter 3 we prove the boundary determination result by using the pseudodifferential calculus. Finally, in Appendix A we prove some elementary results about extending a certain kind of functions and prove the well-posedness of a pseudodifferential heat equation, relevant for the boundary determination. 



\section{CHAPTER 2}

\section{Preliminaries}

\subsection{Notation and basic definitions}

Throughout this section, $(M, g)$ is a compact connected Riemannian manifold of dimension $n$ with boundary, $E$ is a Hermitian vector bundle of rank $m$ over $M$, equipped with a unitary connection $\nabla$. Let $\nu$ be the outward normal to $\partial M$. We also fix a matrix valued potential $Q$, that is a section of the endomorphism bundle of $E$. Moreover, we will denote the sections of $E$ by $C^{\infty}(M ; E)$ or by $\Gamma(E)$ (both notations are standard). Recall that the connection gives rise to a covariant derivative $\nabla: \Gamma(E) \rightarrow \Gamma\left(E \otimes T^{*} M\right)$; moreover, in a trivial vector bundle $E=M \times \mathbb{C}^{m}$ with the standard Hermitian inner product in the fibers, a connection is given by a $m \times m$ matrix of one-forms $A$ and the covariant derivative by $d_{A}=d+A$. We will interchangeably use the following symbols for the covariant derivative: $d_{A}, \nabla_{A}$ and $\nabla$; subscript $A$ here denotes the connection as a formal object, but can also mean the connection 1-form, depending on the context. Furthermore, we will assume the summation convention, where repeated indices mean that we sum over the corresponding index. One can extend the action of the covariant derivative to all $E$-valued differental forms, i.e. sections of $\bigwedge^{p} T^{*} M \otimes E=\Omega^{p}(E)$, by the Lebnitz rule we will still denote this operator by $d_{A}$.

The connection being unitary, means the following compatibility condition:

$$
d\langle u, v\rangle_{E}=\langle\nabla u, v\rangle_{E}+\langle u, \nabla v\rangle_{E}
$$

We can use the Hermitian inner product to define inner product on sections of $E$ :

$$
(u, v)_{L^{2}(M ; E)}=\int_{M}\langle u, v\rangle_{E} d V
$$

where $d V$ is the volume form on $M$ (sometimes omitted from the integrals for simplicity) and more generally on $E$-valued one forms (that is, sections of $C^{\infty}\left(M ; E \otimes T^{*} M\right)$ ), where in local coordinates $\alpha=\alpha_{i} d x^{i}$ and $\beta=\beta_{i} d x^{i}$ :

$$
(\alpha, \beta)_{L^{2}\left(M ; E \otimes T^{*} M\right)}=\int_{M} g^{i j}\left\langle\alpha_{i}, \beta_{j}\right\rangle_{E} d V
$$

In general, we use the notation $d_{A}^{*}$ or $\nabla^{*}$ to denote the formal adjoint of $d_{A}$ acting on vector valued $p$-forms; if $A$ is unitary, then $d_{A}^{*}=(-1)^{(p-1) n+1} \star d_{A} \star$, where $\star$ is the Hodge star acting $\mathbb{C}$-linearly on differential forms with values in $E$ as $\star(\omega \otimes s)=(\star \omega) \otimes s, \omega$ is a differential form and $s$ is a section of $E$. Now using Stokes' theorem one can prove that the following identity holds (see 44 ):

$$
\left(\nabla^{*} u, v\right)_{L^{2}}-(u, \nabla v)_{L^{2}}=-\left(\iota_{\nu} u, v\right)_{L^{2}\left(\partial M ;\left.E\right|_{\partial M}\right)}
$$

where $u$ is an $E$-valued one form and $v$ is a section of $E$.

Now we can define the twisted or the connection Laplacian as

$$
\mathcal{L}_{\nabla}=\nabla^{*} \nabla
$$

We also denote by $\mathcal{L}_{\nabla, Q}=\nabla^{*} \nabla+Q$ (or $\mathcal{L}_{A, Q}$ ) the corresponding Schrödinger operator and $\mathcal{L}_{g, \nabla, Q}$ when we want to emphasise the dependence on the metric. With the assumption that 0 is not a Dirichlet eigenvalue of $\mathcal{L}_{\nabla, Q}$ in $M$, we have the unique solution $u \in H^{1}(M ; E)$ of the 
following Dirichlet problem, in the weak sense:

$$
\left\{\begin{aligned}
\mathcal{L}_{A, Q} u=0, & & \text { in } M \\
u=f, & & \text { on } \partial M
\end{aligned}\right.
$$

for $f \in H^{1}(M ; E)$, by standard elliptic PDE theory. Here, the Sobolev spaces $H^{1}(M ; E)$ and $H_{0}^{1}(M ; E)$ are defined as the completions of $C^{\infty}(M ; E)$ and $C_{0}^{\infty}(M ; E)$ spaces, respectively in the $H^{1}$ norms:

$$
\|u\|_{H^{1}(M ; E)}^{2}=\|u\|_{L^{2}(M ; E)}^{2}+\|\nabla u\|_{L^{2}\left(M ; E \otimes T^{*} M\right)}^{2}
$$

If we denote the unique solution of equation 2.2 by $u_{f}$, we see that we may change $f$ by a $\varphi \in H_{0}^{1}(M)$ and have the same solution. So we define the half Sobolev space as the quotient:

$$
H^{\frac{1}{2}}\left(\partial M ;\left.E\right|_{\partial M}\right):=H^{1}(M ; E) / H_{0}^{1}(M ; E)
$$

which essentially comes from the trace theorems in Sobolev spaces (we lose "half derivative" when restricting to the boundary - see Proposition A.4 for the details). The spaces $H^{-\frac{1}{2}}$ and $H^{-1}$ on $\partial M$ are defined as the duals of $H^{\frac{1}{2}}$ and $H^{1}$ spaces, respectively.

We are aiming to define the DN map as $\Lambda_{A, Q} f=\iota_{\nu} d_{A} u_{f}$ for smooth $f$ and more generally $\Lambda_{A, Q}: H^{\frac{1}{2}} \rightarrow H^{-\frac{1}{2}}$. For any $f, h \in C^{\infty}(M ; E)$, by using $(2.1)$, we can easily see that:

$$
\left\langle\left\langle\Lambda_{A, Q} f, h\right\rangle\right\rangle=\int_{\partial M}\left\langle\iota_{\nu} d_{A} u_{f}, h\right\rangle d S=\int_{M}\left[\left\langle d_{A} u_{f}, d_{A} h\right\rangle+\left\langle Q u_{f}, h\right\rangle\right] d V
$$

where by $\langle\langle\cdot, \cdot\rangle\rangle$ we have denoted the pairing between $H^{-\frac{1}{2}}$ and $H^{\frac{1}{2}}$ and $d S$ is the surface volume form on $\partial M$. Finally, this motivates us to define the DN map via the formula $[2.4$ (see $[\mathbf{7 0}]$ for the case of $E=M \times \mathbb{C}$ and $A=0$ ):

Definition 2.1. The Dirichlet-to-Neumann map or the DN map is defined as the unique bounded $\operatorname{map} \Lambda_{A, Q}: H^{\frac{1}{2}}\left(\partial M ;\left.E\right|_{\partial M}\right) \rightarrow H^{-\frac{1}{2}}\left(\partial M ; E_{\partial M}\right)$, obtained by the following bilinear form:

$$
\left\langle\left\langle\Lambda_{A, Q} f, h\right\rangle\right\rangle=\int_{M}\left[\left\langle d_{A} u_{f}, d_{A} e_{h}\right\rangle+\left\langle Q u_{f}, e_{h}\right\rangle\right] d V
$$

where $f, h \in H^{\frac{1}{2}}\left(\partial M ;\left.E\right|_{\partial M}\right)$ and $e_{h} \in H^{1}(M ; E)$ is any representative of the class of $h$.

Of course, one is left to check all the details of the previous definition check out: we do this in Proposition A.1 in the appendix. Moreover, the above definition works for $Q$ and $A$ in $L^{\infty}$, however we are mostly interested in smooth $A$ and $Q$.

An alternative (not always equivalent) and a more general way (without assuming the wellposedness of (2.2) ) of interpreting the equality of the DN maps is through the equality of Cauchy data spaces. The full Cauchy data space is given by:

$$
\left.C_{A, Q}=\left\{\left(f, \iota_{\nu} d_{A} u\right) \mid \text { there is a } u \text { that solves } 2.2\right)\right\} \subset H^{\frac{1}{2}} \times H^{-\frac{1}{2}}
$$

Here $\iota_{\nu} d_{A} u$ is interpreted in the weak sense, as explained above. Let us point out that in one of the cases that are important for us, that is when $Q=0$, we automatically have that zero is not a Dirichlet eigenvalue of the operator $\mathcal{L}_{A}$, so the DN map is well-defined by Definition 2.1 and the Cauchy data space is given by a graph.

2.1.1. Local expressions for $d_{A}^{*}$ and inner products. For the record, we will write down the explicit formula in local coordinates for the inner product on the differential forms with values in $E$. If two $p$-differential forms with values in $E$ are given locally by $\alpha=\sum \alpha_{I} d x^{I}$ 
and $\beta=\sum \beta_{J} d x^{J}$ then: ${ }^{1}$

$$
\langle\alpha, \beta\rangle_{\Omega^{p}(E)}=\frac{1}{p !} g^{i_{1} j_{1}} \cdots g^{i_{p} j_{p}}\left\langle\alpha_{i_{1} \ldots i_{p}}, \beta_{j_{1} \ldots j_{p}}\right\rangle_{E}
$$

Here $\langle\cdot, \cdot\rangle_{E}$ is the inner product in $E$ and $g^{i j}$ denotes the inverse matrix of the metric in local coordinates $g_{i j}$. Moreover, we state the following formula for the adjoint $d^{*}=(-1)^{p} \star^{-1} d \star=$ $(-1)^{(p-1) n+1} \star d \star$, acting on $p$-forms $:^{2}$

$$
\left(d^{*} \alpha\right)_{\mu_{1} \ldots \mu_{p-1}}=-g_{\mu_{1} \nu_{1}} \cdots g_{\mu_{p-1} \nu_{p-1}} \frac{1}{\sqrt{|\operatorname{det} g|}} \partial_{\nu}\left(\sqrt{|\operatorname{det} g|} g^{\nu \lambda} g^{\nu_{1} \lambda_{1}} \cdots g^{\nu_{p-1} \lambda_{p-1}} \alpha_{\lambda \lambda_{1} \ldots \lambda_{p-1}}\right)
$$

We can combine this information along with the condition that $\int\left\langle d_{A}^{*} \alpha, \beta\right\rangle_{E}=\int\left\langle\alpha, d_{A} \beta\right\rangle_{E}$ for all $p$-forms $\beta$ and $(p+1)$-forms $\alpha$, compactly supported in the interior. Then we get:

$$
d_{A}^{*} \alpha=d^{*} \alpha-\sum_{i_{1}<\ldots<i_{p}} g^{\nu \lambda} A_{\nu} \alpha_{\lambda i_{1} \ldots i_{p}} d x_{i_{1}} \wedge \ldots \wedge d x_{i_{p}}
$$

and as a shorthand we may use $(A, \alpha)=\iota_{A^{\sharp}} \alpha$ for the sum in the above expression. Here $\sharp$ denotes the isomorphism between $T M$ and $T^{*} M$ given by contracting the metric $g$ with a vector. The following identity is also very useful:

$$
d^{*}(f \omega)=f d^{*}(\omega)-\iota_{\nabla f}(\omega)
$$

If the connection is not unitary, then the expression $(-1)^{(p-1) n+1} \star d_{\left(-A^{*}\right)^{\star}}$ gives the formal adjoint in a local trivialisation on $p$-forms, where $A^{*}$ denotes the Hermitian conjugate. We need to emphasise here that, slightly illogically, even if $A$ is non-unitary in this thesis we will use the notation $d_{A}^{*}=(-1)^{(p-1) n+1} \star d_{A} \star$, unless otherwise stated $I^{3}$ We will apply the same principle to all covariant derivatives that appear in the text. Moreover, the above local formula (2.5) still holds for this $d_{A}^{*}$. Then for all $E$-valued 1-forms $u$ and any $A$ :

$$
d_{A}^{*} d_{A} u=d^{*} d u+d^{*}(A u)-(A, d u)-(A, A u)
$$

2.1.2. Yang-Mills connections. As mentioned previously, Yang-Mills (YM) connections are very important in physics and geometry. They satisfy the so called Yang-Mills equations, which are considered as a generalisation of Maxwell's equations in electromagnetism and which provide a framework to write the latter equations in a coordinate-free way (see e.g. [4] or $[\mathbf{1 8}$ for a geometric overview and definitions). The Yang-Mills connections are critical points of the functional:

$$
F_{Y M}(A)=\int_{M}\left|F_{A}\right|^{2} d \omega_{g}
$$

Here $F_{A}=d A+A \wedge A$ is the curvature 2-form with values in the endomorphism bundle of $E$ determined by the map $d_{A}^{2} s=F_{A} \wedge s$ on sections $s \in C^{\infty}(M ; E)$ and $\omega_{g}$ is the volume form. It can then be shown by considering variations of this functional, that the equivalent conditions for $A$ being its critical point are (the Euler-Lagrange equations):

$$
\left(D_{A}\right)^{*} F_{A}=0 \text { and } \quad D_{A} F_{A}=0
$$

\footnotetext{
$\overline{1_{\text {The factor of }}} \frac{1}{p !}$ comes from the fact that we want to have $\left\langle d x^{i_{1}} \wedge \ldots \wedge d x^{i_{p}}, d x^{j_{1}} \wedge \ldots \wedge d x^{j_{p}}\right\rangle=\operatorname{det}\left(g^{i_{k} j_{k}}\right)$. ${ }^{2}$ We are assuming that the tensor representing the form is alternating, i.e. we get a minus sign after swapping any two indices.

${ }^{3}$ The point is that we would like to have $d_{F^{*}(A)}^{*}=F^{-1} d_{A}^{*} F$, for all isomorphisms $F$ of the vector bundle $E$. On the other hand, $F^{-1} d_{A}^{*} F$ will be the formal adjoint if we consider the pulled back inner product structure on $E$ by $F$; in general, it will not be a formal adjoint with respect to the standard inner product structure on $E$.
} 
where $D_{A}=d_{A}^{\text {End }}$ is the induced connection on the endomorphism bundle, given locally by $D_{A} S=d S+[A, S]$ or equivalently by $D_{A} S=\left[d_{A}, S\right]$, where $[\cdot, \cdot]$ denotes the commutator. The second equation in (2.7) is actually redundant, since it is the Bianchi identity.

Yang-Mills connections clearly generalise flat connections, for which the curvature vanishes, i.e. $F_{A}=0$.

Let us motivate the fact we consider Yang-Mills connections in this thesis, by illustrating their significance in other branches of mathematics. They have been a point of unification between pure mathematics and theoretical physics, but moreover have brought a few areas of pure mathematics together, such as e.g. PDE theory and vector bundles over complex projective spaces, or algebraic geometry.

One such example is the proof of bijective correspondence of instantons (self-dual or anti self-dual connections, i.e. the ones for which $\star F_{A}= \pm F_{A}$, where $\star$ is the Hodge star) over $S^{4}$ with certain holomorphic vector bundles over $\mathbb{C P}^{3}$, using the ADHM construction. More generally, one can establish a bijective correspondence of the Hitchin-Kobayashi type (there are a few similar results under the same name) and Uhlenbeck and Yau prove that if $E$ is a stable (in some sense we do not specify) holomorphic vector bundle over a compact Kähler manifold, then $E$ admits a Hermitian-Yang-Mills connection.

Within topology, YM functional $F_{Y M}$ can be regarded as a function on a space with rich topology, the space of connections modulo gauges, in view of Morse theory (much like the energy functional on the space of closed loops) - one can then pose the questions whether one can determine the homotopy group of instantons and other critical points and relate them to the topology of the ambient space. Finally, moduli spaces of instantons were applied by Donaldson to solve some long standing conjectures in the four manifold topology.

Let us also emphasise the nature of the topology of the space of connections modulo gauges: it is infinite dimensional and locally has a unique representative chosen by going to the Coulomb gauge, but globally it is impossible to find such a gauge; Donaldson argues that one of the most important influences of gauge theory is to accustom mathematicians to working with infinite dimensional gauge groups in a comparatively simpler setting, where for example diffeomorphism groups in Riemannian geometry are considered more difficult $\mathbf{1 9}$.

EXAMPLE 2.2 (Yang-Mills connections over Riemann surfaces). We give an idea of the size of the set of YM connections in the simplest non-trivial example of Riemann surfaces. First recall that connections on bundles modulo gauges are classified by their holonomy representation on the so called loop group modulo conjugation (see Kobayashi and Nomizu [43]). In the setting of flat connections, this correspondence simplifies significantly for a Riemann surface $\Sigma$ :

$$
\left.\left\{\rho: \pi_{1}(\Sigma) \rightarrow U(m)\right\} / \text { conj. } \longleftrightarrow \quad \text { unitary flat bundles of rank } m\right\}
$$

since homotopic loops have the same holonomy. The direct map (going left to right) here is the one taking a representation $\rho$ and defining an associated flat bundle via $\widetilde{\Sigma} \times_{\rho} \mathbb{C}^{m}$, where $\widetilde{\Sigma}$ is the universal cover of $\Sigma$ and $\times_{\rho}$ means we identified the two by the diagonal action. Somewhat surprisingly, we may still obtain a correspondence in the case of YM connections, where $\pi_{1}(\Sigma)$ is replaced by a certain central extension $\widehat{\pi}_{1}(\Sigma)$ (see 4 for more details). This has an analogous geometric interpretation: the difference to the flat case is that we now identify homotopic only if they enclose the same area. In particular, for the sphere $S^{2}$ this simplifies, so that we have $\widehat{\pi}_{1}\left(S^{2}\right)=S^{1}$. 
2.1.3. Fixing gauges. In many mathematical problems and physical situations there exist certain degrees of freedom called gauges. More specifically, in our case a gauge is an automorphism of a vector bundle (preserves its structure); then the gauges act on the affine space of connections on this vector bundle by pullback. Here, we make a few remarks about the possible gauges one could use.

EXAMPLE 2.3 (An electromagnetic correspondence). In physics we use the electromagnetic four-potential to describe the electromagnetic field. This potential can be naturally identified (via musical isomorphism, the inverse of $\sharp$ ) with a connection 1-form $A$ on the unitary trivial line bundle over the space-time $\mathbb{R}^{4}$ in the Minkowski metric, so that the actual electromagnetic field is given by the curvature $F=d A$, which is a tensor consisting of six components; the Maxwell's equations then reduce to $d^{*} d A=0$ (see (2.7)).

Recall from classical electromagnetism, if we consider the magnetic potential $\vec{A}$ separately, we would have the field strength $\vec{B}=\nabla \times \vec{A}$. Then we could transform the potential $\vec{A} \mapsto \vec{A}+\nabla f$ and still get the same answer for $\vec{B}$; similarly, the electric field is invariant under addition of $-\frac{\partial f}{\partial t}$ to the electric potential $\phi$ and the correspondence is $A=A_{1} d x^{1}+A_{2} d x^{2}+A_{3} d x^{3}-\phi d t$. In the connection setting above, we have the analogous invariant transform $A \mapsto A+i d f$ for a real function $f$ on $\mathbb{R}^{4}$, which corresponds to the gauge given by $e^{i f}$. This leads to the old physical observation that we do not have a physical meaning of the potential and is a starting point to the Yang-Mills theory which generalises the Maxwell's equations (see [18]).

There are several gauges that have proved to work well in practise, i.e. that fit well into other mathematical formalism in applications. One of them is the Coulomb gauge, which for a connection matrix on a vector bundle, locally asks that $d^{*} A=d^{4}$ The existence of such gauges is proved by Uhlenbeck 83 for vector bundles over unit balls (see also [18]) under a smallness condition on the $L^{p}$ norm of the curvature (for specific values of $p$ ), which locally on a manifold we can always assume if we shrink the neighbourhood sufficiently and then dilate to the unit ball. Most importantly, in such a gauge the Yang-Mills connections satisfy an elliptic partial differential equation with the principal, second order term equal to $\left(d d^{*}+d^{*} d\right) \times I d$, which is clearly elliptic (check in local coordinates).

Another slightly related gauge is the temporal gauge, which we will also make use of - in this gauge, one of the components of the connection vanishes locally (we usually distinguish this variable as "time"). That is, given a local coordinate system $\left(x_{1}, \ldots, x_{n-1}, t\right)=(x, t)$ defined for $t=0$ and a connection matrix $A=A_{i} d x^{i}+A_{t} d t$, we may solve:

$$
\frac{\partial F}{\partial t}(x, t)+A_{t}(x, t) F(x, t)=0 \quad \text { and } \quad F(x, 0)=I d
$$

parametrically smoothly depending on $x$ (the parallel transport equation). Then by definition near $t=0$, we have $A^{\prime}=F^{*}(A)=F^{-1} d F+F^{-1} A F$ satisfying $A_{t}^{\prime}=0$. In this way we prove the following lemma, which will be used frequently throughout the thesis:

Lemma 2.4. Let $A$ and $B$ be two unitary connections on a Hermitian vector bundle $E$ over $M$. Consider the tubular neighbourhood $\partial M \times[0, \epsilon)$ of the boundary for some $\epsilon>0$ and denote the normal distance coordinate (from $\partial M$ ) by $t$. Then $B$ is gauge equivalent to a unitary connection $B^{\prime}$ via an automorphism $F$ of $E$ such that $\left.F\right|_{\partial M}=I d$ and $\left(B^{\prime}-A\right)\left(\frac{\partial}{\partial t}\right)=0$ in the neighbourhood $\partial M \times[0, \delta)$ of the boundary, for some $\delta>0$.

\footnotetext{
${ }^{4}$ This is equivalent to $\nabla \circ \vec{A}=0$ in the case of $\mathbb{R}^{3}$ considered in the previous paragraph.
} 
In particular, if $E=M \times \mathbb{C}^{m}$ we have gauges $F$ and $G$ for $A$ and $B$ respectively with $\left.F\right|_{\partial M}=\left.G\right|_{\partial M}=I d$, such that $A^{\prime}=F^{*} A$ and $B^{\prime}=G^{*} B$ satisfy $A^{\prime}\left(\frac{\partial}{\partial t}\right)=B^{\prime}\left(\frac{\partial}{\partial t}\right)=0$ near the boundary.

Proof. Let us denote $B\left(\frac{\partial}{\partial t}\right)$ by $B_{t}$. Then consider the following first order systems of differential equations, solving the parallel transport equations:

$$
\begin{aligned}
& \frac{\partial F}{\partial t}\left(x^{\prime}, t\right)+A_{t}\left(x^{\prime}, t\right) F\left(x^{\prime}, t\right)=0 \quad \text { with }\left.\quad F\right|_{\partial M}=I d \\
& \frac{\partial G}{\partial t}\left(x^{\prime}, t\right)+B_{t}\left(x^{\prime}, t\right) G\left(x^{\prime}, t\right)=0 \quad \text { with }\left.\quad G\right|_{\partial M}=I d
\end{aligned}
$$

where $F$ and $G$ are $m \times m$ matrices, for $\left(x^{\prime}, t\right) \in U \times[0, \epsilon)$ for some coordinate chart $U \subset \partial M$. This has a unique smooth solution in $U \times\left[0, \delta^{\prime}\right)$, for some positive $\delta^{\prime}$ with $\epsilon>\delta^{\prime}$. Moreover, $F$ and $G$ are unitary, since $B_{t}$ is skew-Hermitian and if we define $H=G F^{-1}$ we have $B^{\prime}:=H^{*} B$ with $B_{t}^{\prime}=A_{t}$ by the equations above:

$$
\frac{\partial H}{\partial t}=\frac{\partial G}{\partial t} F^{-1}+G \frac{\partial F^{-1}}{\partial t}=-B_{t} G F^{-1}+G F^{-1} A_{t}=H A_{t}-B H_{t}
$$

Moreover we see that $H: E_{x} \rightarrow E_{x}$ is defined independently of the chart for $x$ with distance less than $\delta^{\prime}$ to the boundary and $\left(B^{\prime}-A\right)_{t}=0$.

Furthermore, there exists a $\delta>0$ such that $H$ is close to identity in $\partial M \times[0, \delta)$, with $\delta<\delta^{\prime}$. Then we may take a compactly supported function $\varphi$ on $\left[0, \delta^{\prime}\right)$, with $\varphi=1$ on $[0, \delta)$, and define $\rho$ on $M$ by setting $\rho(x, t)=\varphi(t)$ in $\partial M \times\left[0, \delta^{\prime}\right)$ and zero elsewhere. Then we may define the unitary extension $\tilde{H}=e^{\rho \log F}$; clearly $\left.\tilde{H}\right|_{\partial M \times[0, \delta)}=H$ and the globally defined $B^{\prime}:=\tilde{H}^{*} B$ satisfies the requirements.

Moreover, if we perform the above procedure in geodesic polars near a point, with $t$ corresponding to the radial variable $r$ now, we obtain what is called the radial gauge. More generally, given a local vector field $X$, we can always construct a gauge as in Lemma 2.4 by defining $F$ to be parallel transport along the flowlines $\varphi_{t}$ of $X$, by setting $F$ to be identity (initial condition) on a codimension one local submanifold transversal to the flow - we can then always extend $F$ to a local gauge.

In the situation of this Yang-Mills problem, we would like to use the gauge given by Lemma 2.4 in combination with Lemma 8.1 , because the latter one is intimately tied with the DN map (1.1) and allows us to make use of the information packed in the equality $\Lambda_{A}=\Lambda_{B}$ for two connections $A$ and $B$.

\subsection{The integral identity}

Recall the identity 2.5 with $A$ unitary on $E=U \times \mathbb{C}^{m}$ with $U \subset \mathbb{R}^{n}$; we have $\nabla^{*}=d_{A}^{*}=$ $d^{*}-(A, \cdot)$ on one forms, with $(A, \beta)=g^{i j} A_{i} \beta_{j}$ for $\beta$ an $E$-valued one-form. For clarity, we remark that we take the Laplacian with a negative sign, i.e. $\Delta u=d^{*} d u=-|g|^{-1 / 2} \frac{\partial}{\partial x^{j}}\left(|g|^{1 / 2} g_{j k} \frac{\partial u}{\partial x^{k}}\right)$, so our operator is positive definite. Therefore, we can clearly identify the second, the first and the zero order terms in the connection Laplacian. If we let $(A, Q)$ be a pair of a connection and a potential, we will sometimes use the notation of the pair $(X, q)$ to denote the matrix vector field $X$ and the matrix potential $q$ such that:

$$
d_{A}^{*} d_{A}+Q=\Delta+X+q
$$


in local coordinates, or globally if the corresponding bundle is trivial. The relationship between $(A, Q)$ and $(X, q)$ is given by:

$$
X=-2 g^{i j} A_{i} \frac{\partial}{\partial x^{j}} \quad \text { and } \quad q(u)=d^{*} A-(A, A u)+Q(u)
$$

The next lemma computes the adjoint of the DN map, where $Q$ is in $\Gamma($ End $E)$ :

LEMMA 2.5. The following identity holds for smooth $f$ and $g\left(Q^{*}\right.$ is the Hermitian conjugate):

$$
\left(\Lambda_{\nabla, Q} f, g\right)_{L^{2}\left(\partial M ;\left.E\right|_{\partial M}\right)}=\left(f, \Lambda_{\nabla, Q^{*}} g\right)_{L^{2}\left(\partial M ;\left.E\right|_{\partial M}\right)}
$$

Proof. We drop the full notation of $L^{2}(M ; E)$. By using $(2.1)$ we have:

$$
(Q u, v)_{M}+(\nabla u, \nabla v)_{M}=\left(\Lambda_{\nabla, Q} f, g\right)_{\partial M}
$$

where $\mathcal{L}_{\nabla, Q} u=0$ and $\left.u\right|_{\partial M}=f$ and any $v$ such that $\left.v\right|_{\partial M}=g$. If we swap the order of $f$ and $g$ and use the fact that the inner product is Hermitian, along with $v$ being the solution to $\mathcal{L}_{\nabla, Q^{*}} v=0$ and $\left.v\right|_{\partial M}=g$, we get:

$$
\left(Q^{*} v, u\right)_{M}+(\nabla v, \nabla u)_{M}=\left(\Lambda_{\nabla, Q^{*}} g, f\right)_{\partial M}
$$

which after conjugation finishes the proof.

Now we restrict our attention to the trivial vector bundle $E=M \times \mathbb{C}^{m}$ with the connection matrix $A$. We will use the notation $|A|^{2}=g^{i j} A_{i} A_{j}$ - please note this is not a norm, but rather comes from the complex bilinear extension of the metric inner product and that it is endomorphism valued. Also, $\left(A_{j}\right)_{k l}$ will denote the $k l^{\text {th }}$ entry of the matrix $A_{j}$ given by the expansion $A=A_{j} d x^{j}$.

THEOREM 2.6 (Main identity). The following identity holds for two pairs of smooth unitary connections and potentials $\left(A, Q_{A}\right)$ and $\left(B, Q_{B}\right)$, and $f$ and $g$ smooth sections of $\left.E\right|_{\partial M}$ :

$$
\begin{aligned}
\left(\left(\Lambda_{A, Q_{A}}-\Lambda_{B, Q_{B}}\right) f, g\right)_{\partial M}=\left(\left(Q_{A}-Q_{B}+|B|^{2}-|A|^{2}\right) u, v\right)_{M} & \\
& +\int_{M} g^{i j}\left((A-B)_{j}\right)_{k l}\left(u_{l} \frac{\partial \bar{v}_{k}}{\partial x^{i}}-\frac{\partial u_{l}}{\partial x^{i}} \bar{v}_{k}\right)
\end{aligned}
$$

where $u, v \in C^{\infty}(M ; E)$ solve $\mathcal{L}_{A, Q_{A}} u=0$ with $\left.u\right|_{\partial M}=f$ and $\mathcal{L}_{B, Q_{B}^{*}} v=0$ with $\left.v\right|_{\partial M}=g$. Equivalently, for $m=1$ one can write this as:

$$
\begin{aligned}
\left(\left(\Lambda_{A, q_{A}}-\Lambda_{B, Q_{B}}\right) f, g\right)_{\partial M}=\left(\left(Q_{A}-Q_{B}+|B|^{2}-|A|^{2}\right) u, v\right)_{M} & \\
& +\int_{M}\langle u d \bar{v}-\bar{v} d u, B-A\rangle_{g}
\end{aligned}
$$

Proof. As above, we have:

$$
\left(\Lambda_{A, Q_{A}} f, g\right)_{\partial M}=\left(Q_{A} u, v\right)_{M}+\left(d_{A} u, d_{A} v\right)_{M}
$$

and similarly, where $u$ and $v$ as in the statement:

$$
\begin{aligned}
\left(\Lambda_{B, Q_{B}} f, g\right)_{\partial M} & =\left(f, \Lambda_{A_{B}, Q_{B}^{*}} g\right)_{\partial M} \\
& =\left(Q_{B} u, v\right)_{M}+\left(d_{B} u, d_{B} v\right)_{M}
\end{aligned}
$$

So we get by subtracting:

$$
\left(\left(\Lambda_{A, Q_{A}}-\Lambda_{B, Q_{B}}\right) f, g\right)_{\partial M}=\left(\left(Q_{A}-Q_{B}\right) u, v\right)_{M}+\left(d_{A} u, d_{A} v\right)_{M}-\left(d_{B} u, d_{B} v\right)_{M}
$$


We have $(A u, A v)_{M}=-\left(|A|^{2} u, v\right)_{M}$ and $(B u, B v)_{M}=-\left(|B|^{2} u, v\right)_{M}$ and moreover:

$$
(d u,(A-B) v)_{M}+((A-B) u, d v)_{M}=\int_{M} g^{i j}\left((A-B)_{i}\right)_{k l}\left(u_{l} \frac{\partial \bar{v}_{k}}{\partial x^{j}}-\frac{\partial u_{l}}{\partial x^{j}} \bar{v}_{k}\right)
$$

by the skew-Hermitian property of $A$ and $B$, where $u_{l}$ and $v_{k}$ denote the components of the vectors $u$ and $v$. By putting the pieces together, this finishes the proof.

Let us now denote by $E^{\prime}=M \times \mathbb{C}^{m \times m}$ the endomorphism bundle of $E$, carying the natural trace Hermitian inner product $\langle X, Y\rangle=\operatorname{tr}\left(X Y^{*}\right)$. Then we can naturally let the $\mathcal{L}_{A, Q}$ operator act on matrix sections by matrix multiplication! furthermore, one easily shows the similarly extended DN maps for $A_{1}$ and $A_{2}$ on $E^{\prime}$ obtained in this way agree if and only if the usual DN maps for $A_{1}$ and $A_{2}$ agree on $E$ - one just notices that the first claim is the same as the second one applied to all of $n$ column vectors. Therefore, we have a version of the previous identity for matrices, where by capital letter we denote a matrix instead of a vector (we will need it in Section 6.2):

Theorem 2.7 (The identity for matrices). In the notation as in Theorem 2.6, for two smooth sections $F$ and $G$ of $\left.E^{\prime}\right|_{\partial M}$, we have:

$$
\begin{aligned}
\left(\left(\Lambda_{A, Q_{A}}-\Lambda_{B, Q_{B}}\right) F, G\right)_{\partial M}=\left(\left(Q_{A}-Q_{B}+|B|^{2}-\right.\right. & \left.\left.|A|^{2}\right) U, V\right)_{M} \\
& +\left(U\left(d V^{*}\right)-(d U) V^{*}, B-A\right)_{M}
\end{aligned}
$$

where $U, V \in C^{\infty}\left(M ; E^{\prime}\right)$ solve $\mathcal{L}_{A, Q_{A}} U=0$ with $\left.U\right|_{\partial M}=F$ and $\mathcal{L}_{B, Q_{B}^{*}} V=0$ with $\left.V\right|_{\partial M}=G$.

Proof. By re-running the proof of the previous theorem, we easily obtain the result; we use the convenient matrix identities such as $(A U, d V)_{M}=-\left(U\left(d V^{*}\right), A\right)_{M}$ and $(d U, A V)_{M}=$ $\left((d U) V^{*}, A\right)_{M} \cdot$

\subsection{Semiclassical pseudodifferential operators on manifolds}

In this section we briefly review the basics of semiclassical pseudodifferential operators and the associated symbol calculus. The classical theory will be used for the purposes of Chapter 3. where we prove $\Lambda_{A}$ is pseudodifferential operator on the boundary; in Chapter 5, some semiclassical theory (Bessel potentials) is used to prove suitable estimates.

We start by outlining the underlying function spaces, then progress to describing the classical theory and in the end we build up on that by inserting everywhere a small parameter $h$ to define the semiclassical theory.

2.3.1. Distribution spaces, kernels and the semiclassical case. See $6,51,69$ for more details. Let $X \subset \mathbb{R}^{n}$ be an open set. First recall the usual function spaces $C^{\infty}(X)$ and $C_{0}^{\infty}(X)$ and the locally convex space (LCS) topologies on them: the first one is a Fréchet space with the topology of uniform convergence of all derivatives on compact subsets and the second one is an inductive limit of LCS (not a Fréchet space itself and not metrisable). We also use the notation $\mathcal{E}(X)=C^{\infty}(X), \mathcal{D}(X)=C_{0}^{\infty}(X) ; \mathcal{E}_{K}(X)$ will denote the space of smooth function compactly supported in $K$.

The spaces of distributions $\mathcal{D}^{\prime}(X)$ and $\mathcal{E}^{\prime}(X)$ are defined as the duals of $\mathcal{D}(X)$ and $\mathcal{E}(X)$ respectively, and equipped with the weak*-topologies. We have $\mathcal{E}^{\prime}(X) \subset \mathcal{D}^{\prime}(X)$ as the subset of compactly supported distributions.

\footnotetext{
${ }^{5}$ Note $\mathcal{L}_{A, Q}$ is not the same as the connection Laplacian obtained from the standard induced connection $d_{A} U=$ $d U+[A, U]$ on the endomorphism bundle.
} 
Given an open $Y \subset \mathbb{R}^{m}$, an important class of operators $C_{0}^{\infty}(Y) \rightarrow \mathcal{D}^{\prime}(X)$ are given by an integral kernel $K$ such that formally we have:

$$
v \mapsto \int_{Y} K(\cdot, y) v(y) d y
$$

More precisely, if $K \in \mathcal{D}^{\prime}(X \times Y)$, we define the map $K: C_{0}^{\infty}(Y) \rightarrow \mathcal{D}^{\prime}(X)$ by $\langle K v, u\rangle:=$ $\langle K, u \otimes v\rangle$, where $\langle\cdot, \cdot\rangle$ is the distributional pairing. Moreover, by the Schwartz kernel theorem, there is a bijective correspondence between continuous maps $C_{0}^{\infty}(Y) \rightarrow \mathcal{D}^{\prime}(X)$ and distributions in $\mathcal{D}^{\prime}(X \times Y)$.

Given a manifold $M$ of dimension $n$ and a vector bundle $E$ of rank $m$, above notions clearly generalise, with a few subtleties. The space of smooth sections $\mathcal{E}(M, E)$ is topologised by taking the seminorms induced by pushing forward to $\mathbb{R}^{n}$ locally and considering seminorms for any compact $K \subset \mathbb{R}^{n}$; for the compactly supported smooth sections $\mathcal{D}(M, E)$, we notice we can write them as $\cup_{K} \mathcal{E}_{K}(M, E)$ for all $K \subset M$ compact and take the locally convex inductive limit topology, as before.

Now the space of generalised sections is defined by first introducing the "functional dual" $E^{V}:=E^{*} \otimes D_{M}$, where $D_{M}$ is the density bundle $\oint^{6}$ on $M$ and $E^{*}$ is the dual bundle; then we define $\mathcal{D}^{\prime}(M, E)$ as the dual of $\mathcal{D}\left(M, E^{V}\right)$ with the weak*-topology. When $E=M \times \mathbb{C}$, then we get just the space of generalised functions $\mathcal{D}^{\prime}(M)$. Notice that in this way we get $\mathcal{E}(M, E) \subset \mathcal{D}^{\prime}(M, E)$ by applying the generalised section and integrating the density:

$$
s \mapsto \int_{M}\langle\cdot, s\rangle
$$

where on the right hand side we have the natural pairing.

The Schwartz kernel theorem generalises to the manifold and vector bundle setting, by using the function spaces defined in the previous paragraph.

Finally, for the semiclassical case, we introduce the parameter $h \in\left(0, h_{0}\right]$ for some $h_{0}>0$ fixed. The semiclassical Fourier transform, which is just a rescaling of the usual one, is given by:

$$
\left(\mathcal{F}_{h} \phi\right)(\xi)=\hat{\phi}(\xi)=\int_{\mathbb{R}^{n}} e^{-\frac{i}{h} \xi x} \phi(x) d x
$$

for $\phi \in C_{0}^{\infty}\left(\mathbb{R}^{n}\right)$; for $h=1$ we recover the usual transform. Its inverse is given by:

$$
\left(\mathcal{F}_{h}^{-1} \phi\right)(x)=\frac{1}{(2 \pi h)^{n}} \int_{\mathbb{R}^{n}} e^{\frac{i}{h} \xi x} \phi(\xi) d \xi
$$

We will sometimes drop the subscript $h$ depending on context; moreover, $\phi$ will usually be an $h$-dependent function.

For a LCS $\mathcal{V}$, we will denote by $\mathcal{V}_{h}$ the space of functions $\phi:\left(0, h_{0}\right] \rightarrow \mathcal{V}$ which are polynomially bounded, i.e. for each $\phi \in \mathcal{V}_{h}$ and every neighbourhood $\mathcal{U}$ of 0 in $\mathcal{V}$, there exists an $N>0$ and $s>0$, such that $\left\{h^{N} \phi(h) \mid h \in\left(0, h_{0}\right]\right\} \subset s \mathcal{U}$. By doing this, we define the spaces $\mathcal{E}_{h}(X)=C_{h}^{\infty}(X)$ and $\mathcal{D}_{h}(X)=C_{0 h}^{\infty}(X)$.

Furthermore, to define $h$-dependant distributions, we change this definition slightly - for example, we define $\mathcal{D}_{h}^{\prime}(X)$ as the set of all maps $u:\left(0, h_{0}\right] \rightarrow \mathcal{D}^{\prime}(X)$, such that $\left\langle u_{h}, \phi_{h}\right\rangle$ :

\footnotetext{
${ }^{6}$ In order to be able to integrate on a non-orientable $M$, we define the density bundle $D_{M}$ as the complex line bundle with the transition functions equal to absolute value of coordinate change Jacobian. Then we integrate sections of $D_{M}$; note that $D_{M}$ is trivial, e.g. by taking a metric $g$ and the section locally given by $|\operatorname{det} g|^{\frac{1}{2}} d x-$ this quantity also defines a measure on $M$ and as a consequence the space $L^{2}(M)$ is defined. This enables us to identify $\mathcal{D}^{\prime}(M)$ with functionals on compactly supported functions.
} 
$\left(0, h_{0}\right] \rightarrow \mathbb{C}$ is in $\mathbb{C}_{h}$. Here by $u_{h}$ and $\phi_{h}$ we stress the $h$-dependence; the same principle applies to define $\mathcal{E}_{h}^{\prime}(X)$. Note that $X$ can be a manifold.

2.3.2. Pseudodifferential operators. See $[55,73,80]$ for more details. Let us first introduce the usual symbol classes in the pseudodifferential calculus. Let $U \subset \mathbb{R}^{n}$ be open and $N$ an integer - we say that $a \in C^{\infty}\left(U \times \mathbb{R}^{N}\right)$ is a symbol of order $m \in \mathbb{R}$, if for every multiindices $\alpha, \beta$ and any compact $K \subset U$, we have constant $C_{\alpha, \beta, K}>0$ such that

$$
\left|\partial_{\theta}^{\alpha} \partial_{x}^{\beta} a(x, \theta)\right| \leq C_{\alpha, \beta, K}\langle\theta\rangle^{m-|\alpha|}
$$

where $\langle\theta\rangle=\left(1+|\theta|^{2}\right)^{\frac{1}{2}}$, for all $x \in K$ and $\theta \in \mathbb{R}^{N}$. We denote the space of all symbols of order $m$ by $S^{m}\left(X \times \mathbb{R}^{N}\right)$ - it is a Fréchet space with seminorms given by the infimum over all such $C_{\alpha, \beta, K}$. We will use the notation $S^{-\infty}\left(U \times \mathbb{R}^{N}\right):=\cap_{m} S^{m}\left(U \times \mathbb{R}^{N}\right)$.

For $U=X \times X$, where $X \subset \mathbb{R}^{n}$ open, $N=n$ and with the phase function $\Phi(x, y, \xi)=$ $(x-y) \cdot \xi$, given a symbol $a \in S^{m}\left(U \times \mathbb{R}^{n}\right)$, we may define the associated pseudodifferential operator of order $m(\mathrm{PDO}) A: C_{0}^{\infty}(X) \rightarrow C^{\infty}(X)$ :

$$
A u(x)=\frac{1}{(2 \pi)^{n}} \iint e^{i(x-y) \xi} a(x, y, \xi) u(y) d y d \xi
$$

for a function $u \in C_{0}^{\infty}(X)$. Here the integral is considered as an oscillatory integral, i.e. by taking a suitable first order differential operator (there are a few choices) $L=\frac{1+\xi \cdot D_{y}}{1+|\xi|^{2}}$ on $U \times \mathbb{R}^{n}$ for which we have ${ }^{t} L e^{i \Phi}=e^{i \Phi}$, where ${ }^{t} L=\frac{1-\xi \cdot D_{y}}{1+|\xi|^{2}}$ is the formal adjoint of $L ; L$ reduces the order of a symbol by one. Formally integrating by parts enough times, we get a convergent integral with smooth dependance on $x$.

We denote the set of PDOs of order $m$ by $\Psi^{m}(X)$; the set of operators of order $-\infty$ is defined by $\Psi^{-\infty}(X):=\cap_{m} \Psi^{m}(X)$.

Given a PDO $A: C_{0}^{\infty}(X) \rightarrow C^{\infty}(X)$, we may apply the Schwartz kernel theorem to get a kernel $K_{A} \in \mathcal{D}^{\prime}(X \times X)$. Now we state a few basic properties of PDOs:

1. $K_{A} \in C^{\infty}(X \times X \backslash \Delta)$, where $\Delta=\{(x, x) \mid x \in X\}$ is the diagonal.

2. The adjoint ${ }^{t} A$, defined by swapping $x$ and $y$ in $a$, is a PDO; so $A$ extends to $A$ : $\mathcal{E}^{\prime}(X) \rightarrow \mathcal{D}^{\prime}(X)$ by duality.

3. For $u \in \mathcal{E}^{\prime}(X), \operatorname{singsupp}(A u) \subset \operatorname{singsupp}(u)$ (this is called pseudolocality).

To define the composition of PDOs, we need the notion of properly supported PDOs. If $A$ is a $\mathrm{PDO}$, we say it is properly supported if the projections $\Pi_{1}, \Pi_{2}: \operatorname{supp}\left(K_{A}\right) \rightarrow X$ are proper maps (inverse image of a compact set is compact). Such a PDO extends by duality to maps on $C_{0}^{\infty}(X), C^{\infty}(X)$ and $\mathcal{E}^{\prime}(X)$.

Furthermore, we will say a PDO $A$ is smoothing, if it extends to a map $A: \mathcal{E}^{\prime}(X) \rightarrow C^{\infty}(X)$. By a standard lemma, this is equivalent to saying that $K_{A} \in C^{\infty}(X \times X)$, which is also equivalent to $A \in \Psi^{-\infty}(X)$, by the property (2.14) below.

Now given a PDO $A$, we may write it as $A=A_{0}+A_{1}$ where $A_{0}$ properly supported and $A_{1}$ smoothing, by cutting off near the diagonal; then the composition of two properly supported PDOs is a properly supported PDO, which can be seen by choosing appropriate symbols. Furthermore, composition of any two PDOs is defined by taking any two properly supported representatives - one can show this operation is well-defined modulo smoothing.

If we are given a $\mathrm{PDO} A \in \Psi^{m}(X)$, then one can show that it defines continuous maps (similar to differential operators):

$$
A: H_{\text {comp }}^{s}(X) \rightarrow H_{l o c}^{s-m}(X)
$$


Here $H_{l o c}^{s}(X)$ denotes the Sobolev space of distributions in $X$ that are locally in $H^{s}$, equipped with the topology given by seminorms $u \mapsto\|\phi u\|_{s}$ for $\phi \in C_{0}^{\infty}(X)$, while $H_{\text {comp }}^{s}(X)=\mathcal{E}^{\prime}(X) \cap$ $H^{s}(X)$ as a set, equipped with the (locally convex) inductive limit topology induced from $H^{s}(K)$ (distributions in $H^{s}$ with support in a compact $K \subset X$ ).

Finally, given a diffeomorphism $\varphi: X \rightarrow X_{1}$, it can be proved that setting $A_{1} u=A(u \circ \varphi) \circ \varphi_{1}$ defines a pseudodifferential operator of the same order as $A$ on $X_{1}$, where $A$ a PDO on $X$ and $\varphi_{1}=\varphi^{-1}$. This enables us to define a PDO on a manifold:

Definition 2.8. An operator $A: C_{0}^{\infty}(M) \rightarrow C^{\infty}(M)$ is a PDO of order $m$ if for any chart $\varphi: X \subset M \rightarrow X_{1} \subset \mathbb{R}^{n}$, the operator $A_{1}$ induced on $X_{1}$ is a PDO of order $m$. We denote the space of such operators by $\Psi^{m}(M)$.

Moreover, since the classes of symbols are preserved under differemorphisms (changes of variables), we may talk about the space of symbols $S^{m}\left(T^{*} M\right)$ in the cotangent bundle; however, given a PDO $A \in \Psi^{m}(M)$, we only have a well-defined principal symbol $\sigma_{m}(A) \in S^{m}\left(T^{*} M\right) / S^{m-1}$ $\left(T^{*} M\right)$ - the full symbol is not well-defined in general. Similarly, the spaces of classical symbols $C S^{m}\left(T^{*} M\right)$, classical pseudodifferential operators $C \Psi^{m}(M)$ and their principal symbols (now proper functions on $T^{*} M$ ) are well-defined by analogous statements (see the definition of a classical symbol below).

2.3.3. Symbol calculus. Assume that we have a strictly decreasing sequence $m_{j}$ of integers for $j \geq 0$ and assume $a_{j} \in S^{m_{j}}(X, X)$. Then it is an important fact that there exists a symbol $a \in S^{m_{0}}(X, X)$ such that (sometimes referred to as the Borel's construction):

$$
a(x, y, \xi) \sim \sum_{j=0}^{\infty} a_{j}(x, y, \xi)
$$

in the sense that $a-\sum_{j=0}^{r-1} a_{j} \in S^{m_{r}}(X, X)$ for every $r \geq 1$; if we had $a^{\prime}$ with the same expansion then clearly $a-a^{\prime} \in S^{-\infty}(X, X)$.

Given a properly supported PDO $A$, we may define its standard (left) symbol $\sigma_{A}(x, \xi)$ by:

$$
\sigma_{A}(x, \xi)=e_{-\xi}(x) A e_{\xi}(x)
$$

where $e_{\xi}(x)=e^{i x \xi}$; let us denote the space of symbols of order $m$ that are independent of $y$ by $S^{m}(X)$. This clearly generalises the standard formula for a differential operator $A$. It can be proved that $\sigma_{A}(x, \xi)$ defines a symbol for $A$ in the appropriate class - more precisely, given a symbol $a(x, y, \xi)$, there is an asymptotic expansion formula for the standard symbol:

$$
\left.\sigma_{A}(x, \xi) \sim \sum_{\alpha} \frac{1}{\alpha !} \partial_{\xi}^{\alpha} D_{y}^{\alpha} a(x, y, \xi)\right|_{x=y}
$$

This can be proved by writing down the defining equation and Taylor expanding (carefully) in $(y-x)$ and $(\theta-\xi)$, where we integrate over $\theta \in \mathbb{R}^{n}$ and $y \in X$. Finally, this proves there is a bijection between the quotients $\Psi^{m}(X) / \Psi^{-\infty}(X)$ and $S^{m}(X) / S^{-\infty}(X)$.

Sometimes it is useful to consider a special class of symbols - we will call $a_{j} \in S^{j}\left(U \times \mathbb{R}^{n}\right)$ a classical symbol if it is positively homogeneous in $\theta$ of order $j$ (i.e. $a(x, y, r \theta)=r^{j} a(x, y, \theta)$ for $r>0)$. Denote the subspace of such symbols by $C S^{j}\left(U \times \mathbb{R}^{n}\right)$ and the corresponding operators by $C \Psi^{j}(X)$. By cutting off the singularity at $\theta=0$ (by a suitable bump function), we recover the asymptotic expansions in a meaningful way, and the composition of properly supported classical operators can be shown to be classical.

2.3.4. The semiclassical world. See $[\mathbf{2 4}, \mathbf{5 2}, 87$ for more details. Physically, pseudodifferential operators model the correspondence between the classical observables, which are 
just functions on the phase space (standard symbols), and the quantum observables, which are self-adjoint operators on $L^{2}\left(\mathbb{R}^{3}\right)$. For example, the momentum operator corresponds to the classical momentum via conjugating by Fourier transform: $h D_{x}=\mathcal{F}_{h}^{-1} \xi \mathcal{F}_{h}$; the energy function $E=\frac{\xi^{2}}{2 m}+V(x)$ corresponds to the Schrödinger operator $H=\frac{-h^{2}}{2 m} \Delta+V(x)$ (we can add the magnetic part, too).

The $h$ parameter (the Planck constant) comes into play when we want to consider the "classical limit" $h \rightarrow 0$, i.e. this limit corresponds to the limit of quantum mechanics to classical mechanics (Bohr correspondence principle). The mathematical branch that studies this limit is called the semiclassical analysis; in particular it studies the spectrum of the Schrödinger operator as $h \rightarrow 0$.

Sometimes, $h$ is not the Planck constant, but rather a different quantity - e.g. the inverse of the square root of energy in the high-energy spectral problems and can be many other things. Moreover, semiclassical analysis plays a role in the WKB (Wentzel, Kramers, and Brillouin) approximation, which originally constructs approximate (up to order $O\left(h^{\infty}\right)$ ) solutions to the Schrödinger equation, by setting the amplitude ansatz to be an asymptotic sum of the form $a=\sum h^{j} a_{j}$ and the phase function to be a multiple of $h^{-1}$. By gathering powers of $h$ in the Schrödinger equation, we may then inductively solve for $a_{i}$ and obtain the approximate solution. More generally, this method can be applied to differential equations with top order coefficient a multiple of $h$-this type of construction we also apply in Chapter 4 , where we construct the Gaussian Beams.

We will consider the following special class of semiclassical symbols, defined for open $X \subset \mathbb{R}^{n}$, $m, k \in \mathbb{R}$. Let us say that $a \in S^{m, k}(X \times X)$ is polyhomogeneous if $a \in S_{h}^{k}(X \times X)$ and:

$$
a(x, y, \xi ; h) \sim \sum_{j=-m}^{\infty} a_{-j}(x, y, \xi) h^{j}, \quad a_{-j} \in S^{k-m-j}(X \times X)
$$

in the usual sense that $a-\sum_{j=-m}^{N-1} a_{-j} h^{j}=O_{S^{k-m-N}(X \times X)}\left(h^{N}\right) \cdot 7$ Given such a symbol $a \in$ $S^{m, k}(X \times X)$, we may define an operator:

$$
A_{h} u(x)=(2 \pi h)^{-n} \iint e^{\frac{i}{h}(x-y) \xi} a(x, y, \xi ; h) u(y) d y d \xi
$$

that we call a semiclassical pseudodifferential operator of order $(m, k)$ and denote the set of such operators by $\Psi^{m, k}(X)$. We will use the abbreviations $S^{s}(X \times X):=S^{0, s}(X \times X)$ with the corresponding operator class $\Psi^{s}(X)$ and call them operators of order $s$. Note that as defined, $A_{h}$ is map:

$$
A_{h}: C_{0 h}^{\infty}(X) \rightarrow C_{h}^{\infty}(X)
$$

where the asymptotical properties of $A_{h} u_{h}(x)$ as $h \rightarrow 0$ are determined by the so called "stationary phase lemma", which computes the exact asymptotic behaviour of the expression 2.16 - if $a \in h^{-m} S^{k}(X \times X)$, then $A_{h} u_{h} \in h^{\frac{n}{2}+k} C_{h}^{\infty}(X)$. We emphasise at this point that the definition of $\mathcal{V}_{h}$ is important ( $\mathcal{V}$ is a LCS), in the sense that for each $\phi \in \mathcal{V}_{h}$, we really want for every seminorm $p_{\alpha}$ on $\mathcal{V}$ to have an appropriate $N_{\alpha}>0$ with $p_{\alpha}\left(h^{N_{\alpha}} \phi\right)$ bounded.

As before, we have an asymptotic summation lemma, i.e. given a sequence $a_{m_{j}} \in S^{m_{j}}(X \times X)$ with $m_{j}$ strictly decreasing to $-\infty$, there is $a \in S^{0, m_{0}}(X \times X)$ such that:

$$
a(x, y, \xi ; h) \sim \sum_{j=0}^{\infty} a_{m_{j}}(x, y, \xi) h^{j}
$$

\footnotetext{
$\overline{7_{\text {Meaning that }}}$ for every seminorm $p_{\alpha}$ defining the LCS topology on $S^{k-m-N}, p_{\alpha}\left(a-\sum_{j=-m}^{N-1} a_{-j} h^{j}\right)=O\left(h^{N}\right)$ as $h \rightarrow 0$.
} 
Such a semiclassical symbol $a$ is unique modulo smoothing symbols in $S^{-\infty,-\infty}(X \times X)=$ $\cap_{m, k} S^{m, k}(X \times X)$, which is the set of all $b \in C_{h}^{\infty}\left(X \times X \times \mathbb{R}^{n}\right)$ for which we have $b=O_{S^{M}}\left(h^{N}\right)$ for all $M, N$.

Furthermore, we still have the appropriate Schwartz kernel ( $h$ dependent) of a semiclassical $\mathrm{PDO}$, given by the oscillatory integral $K_{A} \in \mathcal{D}_{h}^{\prime}(X \times X)$ :

$$
K_{A}(x, y ; h)=(2 \pi h)^{-n} \int a(x, y, \xi ; h) e^{\frac{i}{h}(x-y) \xi} d \xi
$$

We will call the operator $A$ properly supported in this case, if the projection maps $\Pi_{1}, \Pi_{2}$ : $\operatorname{supp}\left(K_{A}\right) \rightarrow X \times X$ are proper, where $\operatorname{supp}\left(K_{A}\right)$ is defined as the unions of the appropriate sets for each fixed $h$; we may always split $A=A_{0}+A_{1}$ as a sum of a properly supported operator and a smoothing term. Then $A$ extends by duality to maps on the sets $\mathcal{E}_{h}^{\prime}(X), \mathcal{D}_{h}^{\prime}(X)$ and $C_{h}^{\infty}(X)$, and the compositions of such operators are well-defined.

Given a properly supported operator $A$, we define its left (standard) symbol by the formula $a_{L}(x, \xi ; h)=e_{-\xi}(x)\left(A e_{\xi}\right)(x)$, where now $e_{\xi}(x)=e^{\frac{i}{h} \xi x}$; we may always compute the standard symbol via the formula (c.f. 2.15) ):

$$
\left.\sigma_{A}(x, \xi ; h) \sim \sum_{\alpha} \frac{h^{|\alpha|}}{\alpha !} \partial_{\xi}^{\alpha} D_{y}^{\alpha} a(x, y, \xi ; h)\right|_{y=x}
$$

Building on this formula, one can easily obtain the composition calculus of two semiclassical PDOs. More precisely, given $a(x, \xi) \in S^{m, k}$ and $b(x, \xi) \in S^{m^{\prime}, k^{\prime}}$ properly supported, defining $A \in \Psi^{m, k}$ and $B \in \Psi^{m^{\prime}, k^{\prime}}$, we have the following expression for the standard symbol $c$ of $C:=A \circ B \in \Psi^{m+m^{\prime}, k+k^{\prime}}$ :

$$
c(x, \xi) \sim \sum_{\alpha} \frac{h^{|\alpha|}}{\alpha !} \partial_{\xi}^{\alpha} a(x, \xi) D_{x}^{\alpha} b(x, \xi)
$$

As in the ordinary PDO case, it can also be shown that difeomorphisms preserve the symbol classes. Moreover, we may extend the theory to manifolds - this means that for a manifold $M$, we have the notion of $\Psi^{m, k}(M)$, the space of pseudodifferential operators of order $(m, k)$ (c.f. Definition 2.8). Also, we may define $S^{m, k}\left(T^{*} M\right)$ to be the space of left (standard) symbols; then the principal symbol $\sigma_{m, k}(A)$ of an operator $A \in \Psi^{m, k}(M)$ is well-defined as an element of the quotient $S^{m, k}\left(T^{*} M\right) / S^{m-1, k-1}\left(T^{*} M\right)$. We denote the space of operator of order $(0, s)$ by $\Psi^{s}(M)$ and call them operators of order $s$.

We record the following fact for future purposes: given smooth cut-off functions $\phi, \psi \in$ $C_{0}^{\infty}(M)$ with disjoint supports and $A \in \Psi^{m, k}(M)$, then we have the following consequence of pseudolocality and the non-stationary phase lemma (c.f. item 3. in Subsection 2.3.2):

$$
\phi A \psi \in \Psi^{-\infty,-\infty}(M)
$$





\section{CHAPTER 3}

\section{Boundary determination for a connection and a matrix potential}

In this chapter, we prove that if we put the connection in a suitable gauge and "normalise" the metric appropriately, we may determine the full Taylor series of a connection, metric and matrix potential from the DN map on a vector bundle with $m>1$. The case of $m=1$ was already considered in [21] (Section 8) and this section generalises the result proved there. The approach is based on constructing a factorisation of the operator $d_{A}^{*} d_{A}+Q$ modulo smoothing, from which we deduce that $\Lambda_{g, A, Q}$ is a pseudodifferential operator of order one whose full symbol determines the mentioned Taylor series.

\subsection{PDOs on vector bundles}

Before going into proofs, let us briefly lay out some of the notation that goes into pseudodifferential operators on vector bundles over manifolds (see [55] and [54] for more details). Firstly, the local symbol calculus and the semiclassical symbol calculus that we developed in Subsection 2.3.3 and Subsection 2.3.4 carries over to matrix valued symbols (starting from (2.12)); in particular, the asymptotic summation properties clearly generalise to this case.

So given $X \subset \mathbb{R}^{n}$ open, $k, l \in \mathbb{N}$ and $m, k \in \mathbb{R}$, we have the left symbol classes (and more generally, $(x, y)$-dependant symbols $) S^{m, k}\left(X ; \mathbb{C}^{l k}\right)$ of $l$ by $k$ matrices, whose entries are symbols in $S^{m, k}(X)$ - this symbol class yields a map $A: C_{0 h}^{\infty}\left(X, \mathcal{C}^{k}\right) \rightarrow C_{h}^{\infty}\left(X, \mathbb{C}^{l}\right)$ via the formula (2.16), which we define to belong to the class $\Psi^{m, k}\left(X ; \mathbb{C}^{l k}\right)$. We will also say that $A$ is $\mathbb{C}^{l k}$-valued semiclassical PDO on $X$.

Then given a Riemannian manifold $M$ and vector bundles $E$ and $F$ over $M$, we say that a linear map $A: C_{0 h}^{\infty}(M ; E) \rightarrow C_{h}^{\infty}(M ; F)$ is a semiclassical PDO of order $(m, k)$ if for every chart and some trivialisations of $E$ and $F$ over this chart, the induced map in the local chart is in $\Psi^{m, k}$ (c.f. Definition 2.8). We write $A \in \Psi^{m, k}(M ; E, F)$ for the space of semiclassical PDOs of order $(m, k)$ and define the space of smoothing operators $\Psi^{-\infty,-\infty}(M ; E, F)=\cap_{m, k} \Psi^{m, k}(M ; E, F)$; we will abbreviate $\Psi^{m, k}(X ; E):=\Psi^{m, k}(X ; E, E)$. Such an operator extends by duality to a map $A: \mathcal{E}_{h}^{\prime}(X, E) \rightarrow \mathcal{D}_{h}^{\prime}(X, F)$ (the transpose ${ }^{t} A$ is defined by taking the transpose of the symbol $a$ and swapping $x$ and $y)$.

For the ordinary (not $h$-dependent) PDO theory over vector bundles, we may just formally "erase" the $h$-dependence - it is clear enough how this theory can be developed. In fact, for this chapter we will specialise in the ordinary PDO theory.

Care should be taken when considering the composition calculus, since commutation properties of matrices jumps into play. More precisely, we have the following composition formula (see the proof of Theorem 4.3 in $[\mathbf{8 0}]$ ), which computes the symbol $c$ modulo $S^{-\infty,-\infty}$ of the composition $C=A \circ B$ of two matrix valued pseudodifferential operators $A$ ( $k$ by $l)$ and $B(l$ by $r$ ) with symbols $a$ and $b$, respectively:

$$
c(x, \xi) \sim \sum_{\alpha} \frac{h^{|\alpha|}}{\alpha !} \partial_{\xi}^{\alpha} a(x, \xi) D_{x}^{\alpha} b(x, \xi)
$$


Finally, we remark that the globally defined principal symbol of a semiclassical PDO $A \in$ $\Psi^{m, k}(M ; E, F)$ is a well-defined element of the quotient

$$
\sigma_{m, k}(A) \in S^{m, k}\left(M ; \operatorname{Hom}\left(\pi^{*}(E), \pi^{*}(F)\right)\right) / S^{m-1, k-1}\left(M ; \operatorname{Hom}\left(\pi^{*}(E), \pi^{*}(F)\right)\right)
$$

where $\pi: T^{*} M \rightarrow M$ denotes the projection, $\pi^{*}$ is the pullback and Hom is the homomorphism bundle.

REMARK 3.1. One of the things that fails to hold for matrix pseudodifferential operators and holds for scalar ones, is that commutation decreases degree of the operator by one. However, the following formula still holds if $c$ denotes the symbol of $C=[A, B]$ (commutator bracket) and $a \in S^{m, k}\left(X ; \mathbb{C}^{l^{2}}\right), b \in S^{m^{\prime}, k^{\prime}}\left(X ; \mathbb{C}^{l^{2}}\right)$ are the symbols of $A, B$, respectively:

$$
c(x, \xi)=[a, b](x, \xi)+\frac{h}{i}\{a, b\}(x, \xi) \quad \text { modulo } S^{m+m^{\prime}-2, k+k^{\prime}-2}
$$

where $\{a, b\}(x, \xi)=\sum_{j=1}^{n}\left(\frac{\partial a}{\partial \xi_{j}} \frac{\partial b}{\partial x^{j}}-\frac{\partial b}{\partial \xi_{j}} \frac{\partial a}{\partial x^{j}}\right)$ denotes the matrix valued Poisson bracket.

\subsection{Boundary determination}

We are now ready for the main proofs - we assume that $(M, g)$ is a compact $n$-dimensional manifold with non-empty boundary $N=\partial M$ and $E=M \times \mathbb{C}^{m}$ a Hermitian vector bundle with a unitary connection $A$ and $Q$ an $m \times m$ matrix whose entries are smooth functions. We will be working in semigeodesic coordinates near $\partial M$ and we denote by $x^{n}$ the normal coordinate and by $x^{\prime}=\left(x^{1}, x^{2}, \ldots x^{n-1}\right)$ the local coordinates in $\partial M$. Furthermore, we have in these coordinates that $g=\sum_{\alpha, \beta} g_{\alpha \beta}(x) d x^{\alpha} d x^{\beta}+\left(d x^{n}\right)^{2}$; also, in what follows the summation convention will be used to sum over repeated indices and when using Greek indices $\alpha$ and $\beta$, the summation will always be assumed to go over $1, \ldots, n-1$. We use the notation $D_{x^{j}}=-i \partial_{x^{j}}=-i \frac{\partial}{\partial x^{j}}$ and $|g|=\operatorname{det}\left(g_{i j}\right)=\operatorname{det}\left(g_{\alpha \beta}\right)$. We start by proving an analogue of Lemma 8.6 in [21] and Proposition 1.1 in $[\mathbf{5 0}$.

Lemma 3.2. Let us assume A satisfies condition (3.12). There exists a $\mathbb{C}^{m \times m}$-valued pseudodifferential operator $B\left(x, D_{x^{\prime}}\right)$ of order one on $\partial M$, depending smoothly on $x^{n} \in[0, T]$ for some $T>0$, such that the following factorisation holds:

$$
d_{A}^{*} d_{A}+Q=\left(D_{x^{n}} \times I d+i E(x) \times I d-i B\left(x, D_{x^{\prime}}\right)\right)\left(D_{x^{n}} \times I d+i B\left(x, D_{x^{\prime}}\right)\right)
$$

modulo smoothing, where $E(x)=-\frac{1}{2} g^{\alpha \beta}(x) \partial_{x^{n}} g_{\alpha \beta}(x)$.

Proof. First of all, we have that:

$$
\left(d_{A}^{*} d_{A}+Q\right) u=\Delta_{g}(u)-2 g^{j k} A_{j} \frac{\partial u}{\partial x^{k}}+\left(d^{*} A\right) u-g^{j k} A_{j} A_{k} u+Q u
$$

where $A=A_{i} d x^{i}$. Furthermore, we have

$$
\Delta_{g}=D_{x^{n}}^{2}+i E D_{x^{n}}+Q_{1}+Q_{2}
$$

where

$$
Q_{1}\left(x, D_{x^{\prime}}\right)=-i\left(\frac{1}{2} g^{\alpha \beta}(x) \partial_{x^{\alpha}} \log |g|(x)+\partial_{x^{\alpha}} g^{\alpha \beta}(x)\right) D_{x^{\beta}} \quad \text { and } \quad Q_{2}\left(x, D_{x^{\prime}}\right)
$$

$$
=g^{\alpha \beta} D_{x^{\alpha}} D_{x^{\beta}}
$$

We denote the symbols of $Q_{1}$ and $Q_{2}$ by $q_{1}$ and $q_{2}$ respectively and define $G=\left(d^{*} A\right)-g^{\alpha \beta} A_{\alpha} A_{\beta}+$ $Q$. Thus by using $(3.3)$, we can rewrite 3.2 as

$$
B^{2}-E B+i\left[D_{x^{n}} \times I d, B\right]=Q_{1} \times I d+Q_{2} \times I d-2 g^{\alpha \beta} A_{\alpha} \partial_{x^{\beta}}+G
$$


modulo smoothing. Moreover, by taking symbols we obtain:

$$
\sum_{\alpha \geq 0} \frac{1}{\alpha !} \partial_{\xi^{\prime}}^{\alpha} b D_{x^{\prime}}^{\alpha} b-E b+\partial_{x^{n}} b-q_{1} \times I d-q_{2} \times I d+2 i g^{\alpha \beta} A_{\alpha} \xi_{\beta}-G=0
$$

modulo $S^{-\infty}$, where $b$ is the symbol of $B$ and we have used (3.1) and Remark 3.1. Let us put $b\left(x, \xi^{\prime}\right)=\sum_{j \leq 1} b_{j}\left(x, \xi^{\prime}\right)$, where $b_{j}$ is homogeneous of order $j$ in $\xi^{\prime}$. We may then determine $b_{j}$ inductively, starting from degree two in (3.4):

$$
\left(b_{1}\right)^{2}=q_{2}
$$

so we may set $b_{1}=-\sqrt{q_{2}} \times I d$ (this sign will be important later) and $q_{2}=g^{\alpha \beta} \xi_{\alpha} \xi_{\beta}$. Next, we have:

$$
\begin{aligned}
b_{0} & =\frac{1}{2 \sqrt{q_{2}}}\left(\partial_{x^{n}} b_{1}-E b_{1}-q_{1} \times I d+2 i g^{\alpha \beta} A_{\alpha} \xi_{\beta}+\nabla_{\xi^{\prime}} b_{1} \cdot \nabla_{x^{\prime}} b_{1}\right) \\
b_{-1} & =\frac{1}{2 \sqrt{q_{2}}}\left(\partial_{x^{n}} b_{0}-E b_{0}-G+\sum_{0 \leq j, k \leq 1, j+k=|K|} \frac{\partial_{\xi^{\prime}}^{K} b_{j} D_{x^{\prime}}^{K} b_{|K|-j}}{K !}\right) \\
b_{m-1} & =\frac{1}{2 \sqrt{q_{2}}}\left(\partial_{x^{n}} b_{m}-E b_{m}+\sum_{m \leq j, k \leq 1, j+k=|K|+m} \frac{\partial_{\xi^{\prime}}^{K} b_{j} D_{x^{\prime}}^{K} b_{k}}{K !}\right)
\end{aligned}
$$

where the last equation holds for all $m \leq-1$. Therefore we obtain $b \in S^{1}$ and hence $B \in \Psi^{1}$ as well, such that 3.2 holds.

We have established the existence of the factorisation 3.2 and now it is time to use it to prove facts about the DN map. The following claim is analogous to Proposition 1.2 in [50 the main difference is that now we are using matrix valued pseudodifferential operators, so we need to make sure that appropriate generalisations hold.

Proposition 3.3. The DN map $\Lambda_{g, A, Q}$ is a $\mathbb{C}^{m \times m}$-valued pseudodifferential operator of order one on $\partial M$ and satisfies $\Lambda_{g, A, Q} \equiv-\left.B\right|_{\partial M}$ modulo smoothing.

Proof. Assume without loss of generality that $A$ satisfies condition $(3.12)$ (see the paragraph after this Proposition). Let us take $f \in H^{\frac{1}{2}}\left(\partial M ; \mathbb{C}^{m}\right)$ and $u \in \mathcal{D}^{\prime}\left(M ; \mathbb{C}^{m}\right)$ that solves the Dirichlet problem $\mathcal{L}_{A, Q} u=0$ with $\left.u\right|_{\partial M}=f$. Then by Lemma 3.2 we obtain the following equivalent local system:

$$
\begin{aligned}
\left(D_{x^{n}} \times I d+i B\right) u & =v \quad \text { with }\left.\quad u\right|_{x^{n}=0}=f \\
\left(D_{x^{n}} \times I d+i E \times I d-i B\right) v & =h \in C^{\infty}\left([0, T] \times \mathbb{R}^{n-1} ; \mathbb{C}^{m}\right)
\end{aligned}
$$

for some $T>0$ and a local coordinate system $x^{\prime}=\left(x^{1}, \ldots, x^{n-1}\right)$ at $\partial M$. By 3.10 and Remark 1.2 from Treves $8 \mathbf{8 0}$, we may furthermore assume that $u \in C^{\infty}\left([0, T] ; \mathcal{D}^{\prime}\left(\mathbb{R}^{n-1} ; \mathbb{C}^{m}\right)\right)$.

Then writing $t=T-x^{n}$, we may view the equation (3.10) as backwards generalised heat equation:

$$
\partial_{t} v-(B-E \times I d) v=-i h
$$

and by standard elliptic interior regularity we obtain that $u$ is smooth and hence, so is $\left.v\right|_{x^{n}=T}$. Since the principal symbol of $B$ is negative, by Lemma A.11 it satisfies Condition A.9 (the basic hypothesis of a well-posed heat equation - see Section A.2 for more details) and so the solution operator for this equation is smoothing by Theorem 1.2 in Chapter 3 of $[\mathbf{8 0}]$. Thus $v \in C^{\infty}\left([0, T] \times \mathbb{R}^{n-1} ; \mathbb{C}^{m}\right)$. 
Let us set $R f:=\left.v\right|_{\partial M}$ - the above argument shows $R$ is a smoothing operator and also $\left.D_{x^{n}} u\right|_{\partial M}=-\left.i B u\right|_{\partial M}+R f$. Therefore $\left.\left.\partial_{x^{n}} u\right|_{\partial M} \equiv B u\right|_{\partial M}$ modulo smoothing, which proves the claim.

The final step in this procedure is to express the Taylor series of $g, A, q$ in terms of the symbols $\left\{b_{j} \mid j \leq 1\right\}$ that we obtained in Proposition 3.3. However, before proving such a result, we need to "normalise" the metric and the connection - here we refer to our Lemma 2.4 and to Lemma 2.1 (b) from 47 : there exists an automorphism $F$ of $E$ such that $\left.F\right|_{\partial M}=I d$ and a positive function $c$ on $M$, with $\left.c\right|_{\partial M}=1$ and $\left.\partial_{\nu} c\right|_{\partial M}=0(\nu$ is the outer normal) such that $\tilde{A}=F^{*}(A)$ and $\tilde{g}=c^{-1} g$ satisfy:

$$
\begin{aligned}
\tilde{\partial}_{x^{n}}^{j}\left(\tilde{g}_{\alpha \beta} \tilde{\partial}_{x^{n}} \tilde{g}^{\alpha \beta}\right)\left(x^{\prime}, 0\right) & =0 \text { for } j \geq 1 \\
\tilde{A}_{n}\left(x^{\prime}, \tilde{x}_{n}\right) & =0
\end{aligned}
$$

where by $\left(x^{\prime}, \tilde{x}^{n}\right)$ we have denoted the $\tilde{g}$-boundary normal coordinates and $\tilde{\partial}_{x^{n}}$ denotes $\partial_{\tilde{x}^{n}}$; (3.12) holds for all sufficiently small $\tilde{x}^{n}$, i.e. in a neighbourhood of the boundary. Also notice that the condition (3.11) is equivalent to $\left.\mathcal{L}_{\tilde{N}}^{j} \tilde{H}\right|_{\partial M}=0$ for $j \geq 1$, as stated in 47 ; here $\tilde{N}=\tilde{\partial}_{x^{n}}$, $\mathcal{L}$ is the Lie derivative and $\tilde{H}$ is the mean curvature of the hypersurfaces given by setting $\tilde{x}^{n}$ equal to constant. Then by the invariance property of the DN map, we have $\Lambda_{g, A, Q}=\Lambda_{\tilde{g}, \tilde{A}, \tilde{Q}}$ for $Q_{c}=c^{\frac{n-2}{4}} \Delta_{g}\left(c^{-\frac{n-2}{4}}\right) \times I d$ and $\tilde{Q}=c\left(F^{-1} Q F+Q_{c}\right)=c\left(F^{*}(Q)+Q_{c}\right)$. We will call a triple $\{g, A, Q\}$ that satisfies conditions (3.11) and (3.12) normalised. Moreover, we will use the notation $f_{1} \simeq f_{2}$ to denote that $f_{1}$ and $f_{2}$ have the same Taylor series (as in [21]).

Theorem 3.4. Assume $M$ satisfies $\operatorname{dim} M=n \geq 3$ and the triple $\{g, A, Q\}$ is normalised. Let $W \subset \partial M$ open, with a local coordinate system $\left\{x^{1}, \ldots, x^{n-1}\right\}$ and let $\left\{b_{j} \mid j \leq 1\right\}$ denote the full symbol of $B$ (see Lemma 3.2) in these coordinates. At any point $p \in W$, the full Taylor series of $g, A$ and $Q$ can be determined by the symbols $\left\{b_{j}\right\}$ by an explicit formula.

In particular, if $\Lambda_{g_{1}, A_{1}, Q_{1}}=\Lambda_{g_{2}, A_{2}, Q_{2}}$ and we assume that $\left\{g_{i}, A_{i}, Q_{i}\right\}$ are normalised for $i=1,2$, then $g_{1} \simeq g_{2}, A_{1} \simeq A_{2}$ and $Q_{1} \simeq Q_{2}$. Moreover, if $\Lambda_{g_{1}, A_{1}, Q_{1}}=\Lambda_{g_{2}, A_{2}, Q_{2}}$ and $g_{1} \simeq g_{2}$ on all of $\partial M$, then we also have $\tilde{A}_{1} \simeq \tilde{A}_{2}$ and $\tilde{Q}_{1} \simeq \tilde{Q}_{2}$, for $\tilde{A}_{i}=F_{i}^{*}\left(A_{i}\right)$ and $\tilde{Q}_{i}=F_{i}^{*}\left(Q_{i}\right)$ for $i=1,2$; here $F_{i}$ are automorphisms of $E$ satisfying $\left.F_{i}\right|_{\partial M}=I d$ and such that $\tilde{A}_{i}$ satisfy condition 3.12 for $i=1,2$.

Proof. Since we have:

$$
\partial_{x^{n}} g_{\alpha \beta}=-\left(g_{\alpha \rho} \partial_{x^{n}} g^{\rho \gamma}\right) g_{\gamma \beta}
$$

it suffices to determine the inverse matrix $g^{\alpha \beta}$ and its normal derivatives. By the formula 3.5, we have that $b_{1}^{2}=-g^{\alpha \beta} \xi_{\alpha} \xi_{\beta}$ determines $\left.g^{\alpha \beta}\right|_{\partial M}$.

If we write $\omega=\frac{\xi^{\prime}}{\left|\xi^{\prime}\right|_{g}}$ and use the notation:

$$
k^{\alpha \beta}=\partial_{x^{n}} g^{\alpha \beta}-\left(g_{\gamma \delta} \partial_{x^{n}} g^{\gamma \delta}\right) g^{\alpha \beta}
$$

then we may rewrite $(3.6)$ as follows:

$$
b_{0}=i g^{\alpha \beta} A_{\alpha} \omega_{\beta}-\frac{1}{4} k^{\alpha \beta} \omega_{\alpha} \omega_{\beta} \times I d+T_{0}\left(g^{\alpha \beta}\right)
$$

where $T_{0}$ depends only on $\left.g^{\alpha \beta}\right|_{\partial M}$, which is already explicitly determined.

Thus, by plugging in $\pm \omega$, we may recover $A_{\alpha}$ and $k^{\alpha \beta}$; it is not hard to see that:

$$
k^{\alpha \beta} g_{\alpha \beta}=(2-n) \partial_{x^{n}} g^{\alpha \beta} g_{\alpha \beta}
$$


and we may therefore write:

$$
\partial_{x^{n}} g^{\alpha \beta}=k^{\alpha \beta}+\frac{1}{2-n}\left(k^{\rho \gamma} g_{\rho \gamma}\right) g^{\alpha \beta}
$$

In the next step we will use the notation $l^{\alpha \beta}=\frac{1}{4} \partial_{x^{n}} k^{\alpha \beta}+Q g^{\alpha \beta}$. Then we may rewrite (3.7) as:

$$
b_{-1}=\frac{1}{2 \sqrt{q_{2}}}\left(i g^{\alpha \beta}\left(\partial_{x^{n}} A_{\alpha}\right) \omega_{\beta}-l^{\alpha \beta} \omega_{\alpha} \omega_{\beta}\right)+T_{1}\left(g^{\alpha \beta}, \partial_{x^{n}} g^{\alpha \beta}, A_{\alpha}\right)
$$

where $T_{1}$ is an expression that depends only on $g^{\alpha \beta}, \partial_{x^{n}} g^{\alpha \beta}$ and $A_{\alpha}$ which have already been explicitly determined. Therefore, we may recover $l^{\alpha \beta}$ and $\partial_{x^{n}} A_{\alpha}$. Now, inductively we may prove the formula:

$$
\begin{aligned}
b_{m-1}=\left(\frac{1}{2 \sqrt{q_{2}}}\right)^{m-1}\left(i g^{\alpha \beta} \partial_{x^{n}}^{|m-1|} A_{\alpha} \omega_{\beta}-\partial_{x^{n}}^{|m|} l^{\alpha \beta} \omega_{\alpha} \omega_{\beta}\right) & \\
& +T_{m-1}\left(g^{\alpha \beta}, \ldots, \partial_{x^{n}}^{|m-1|} g^{\alpha \beta}, A_{\alpha}, \ldots, \partial_{x^{n}}^{|m|} A_{\alpha}, Q, \ldots, \partial_{x^{n}}^{|m+1|} Q\right)
\end{aligned}
$$

for $m \leq-1$, where $T_{m-1}$ only depends on the quantities in the bracket. Therefore, by induction we may explicitly determine $\partial_{x^{n}}^{j} l^{\alpha \beta}$ and $\partial_{x^{n}}^{j} A_{\alpha}$ for all $j \geq 0$.

Finally, we claim that we may inductively recover $\partial_{x^{n}}^{j+2} g^{\alpha \beta}$ and $\partial_{x^{n}}^{j} Q$ for any $j \geq 0$; let us also denote $S_{j}=g_{\alpha \beta} \partial_{x^{n}}^{j} g^{\alpha \beta}$. For the base case $j=0$, notice that $\partial_{x^{n}}\left(g_{\alpha \beta} \partial_{x^{n}} g^{\alpha \beta}\right)=0$, which implies that $S_{2}=-\partial_{x^{n}} g_{\alpha \beta} \partial_{x^{n}} g^{\alpha \beta}$, i.e. we know $S_{2}$.

Therefore, since we know $l^{\alpha \beta}$, we may also explicitly determine $\frac{1}{4} \partial_{x^{n}}^{2} g^{\alpha \beta} \times I d+Q g^{\alpha \beta}=: P_{0}^{\alpha \beta}$. This implies:

$$
P_{0}^{\alpha \beta} g_{\alpha \beta}=(n-1) Q+\frac{1}{4} S_{2}
$$

from which we easily infer the knowledge of $Q$ and hence also of $\partial_{x^{n}}^{2} g^{\alpha \beta}$.

For the inductive step, we may do something very similar: we have that for $j \geq 1$, the quantity $P_{j}^{\alpha \beta}=\frac{1}{4} \partial_{x^{n}}^{j+2} g^{\alpha \beta}+\left(\partial_{x^{n}}^{j} Q\right) g^{\alpha \beta}$ is determined, since the condition $\partial_{x^{n}}^{j+1}\left(g_{\alpha \beta} \partial_{x^{n}} g^{\alpha \beta}\right)=0$ determines $S_{j+2}$ by previously reconstructed quantities. Then by the formula:

$$
P_{j}^{\alpha \beta} g_{\alpha \beta}=(n-1) \partial_{x^{n}}^{j} Q+\frac{1}{4} S_{j+2}
$$

we may determine $\partial_{x^{n}}^{j} Q$ and thus, $\partial_{x^{n}}^{j+2} g^{\alpha \beta}$ as well. This completes the proof of the induction and of the theorem, since two formal expansions of the same operator in terms of classical symbols that agree modulo $S^{-\infty}$, must also be congruent.

Let us emphasise that a key role in the above generalisations to the vector case is played by the fact that the operator $d_{A}^{*} d_{A}+Q$ has a principal symbol that is a scalar multiple of identity; the necessary algebra then unveils in much the same way as in the scalar case. A couple of remarks are in place.

REMARK 3.5 (Boundary determination for surfaces). There are a few reasons to exclude the case $\operatorname{dim} M=2$ in Theorem 3.4. To start with, after the proof of Proposition 1.3 in [50], the authors (considering the case $E=M \times \mathbb{C}, A=0$ and $Q=0$ ) remark that all the symbols of $B$ satisfy $b_{j}=0$ for $j \leq 0$ (easily checked for $b_{0}$ by direct computation and for the rest by induction); in other words, if we choose $b_{1}=-\xi_{1} \sqrt{g^{11}}$, the factorisation $(3.2$ becomes a factorisation into honest differential operators where $B=-\sqrt{g^{11}} D_{x^{1}}$, which is in compliance with the additional conformal symmetry of the Calderón problem for surfaces. Secondly, the equation (3.13) clearly fails to hold when $n=2$ - in that case $k^{11}=0$ clearly so there is no extra information from this expression. However, when we introduce a connection and a potential, 
one can show that (choose $b_{1}=-\xi_{1} \sqrt{g^{11}}$ again):

$$
\begin{aligned}
b_{0} & =i \sqrt{g^{11}} A_{1} \\
2 \xi_{1} b_{-1} & =\partial_{x^{2}} A_{1}-\left(\partial_{x^{1}} \sqrt{g^{11}}\right) A_{1}-\frac{Q}{\sqrt{g^{11}}}
\end{aligned}
$$

Thus, the DN map determines the values of $g_{11}$ and $A_{1}$ at the boundary (recall that $A_{2}=0$ in a neighbourhood of the boundary). Therefore, we may also determine $\partial_{x^{2}} A_{1}-\frac{Q}{\sqrt{g^{11}}}$ from the expression for $b_{-1}$ and so if $Q=0$, we determine the normal derivative of order one $\partial_{x^{2}} A_{1}$ - we will need this fact for a later application. If we go on to compute $b_{-2}$, we see that it suffices to determine $\left.\partial_{x^{2}} g_{11}\right|_{\partial M}$ to compute derivatives $\left.\partial_{x^{2}}^{j} A_{1}\right|_{\partial M}$ of all orders $j \geq 2$; however, again, we know we cannot possibly determine $\left.\partial_{x^{2}} g_{11}\right|_{\partial M}$ due to the additional conformal symmetry of the problem in two dimensions.

REMARK 3.6 (Local boundary determination). If we assume that $\Gamma \subset \partial M$ is open and $\left.\Lambda_{g_{1}, A_{1}, Q_{1}}(f)\right|_{\Gamma}=\left.\Lambda_{g_{2}, A_{2}, Q_{2}}(f)\right|_{\Gamma}$ for all $f \in C_{0}(\Gamma)$ and that the coresponding quantities are normalised, then by the local nature of the above argument in Theorem 3.4, we have that: $\left.\left.g_{1}\right|_{\Gamma} \simeq g_{2}\right|_{\Gamma},\left.\left.A_{2}\right|_{\Gamma} \simeq A_{2}\right|_{\Gamma}$ and $\left.\left.Q_{1}\right|_{\Gamma} \simeq Q_{2}\right|_{\Gamma}$.

We end this chapter with an observation that what we proved so far may be translated to the setting of an arbitrary vector bundle $E$ over $M$, rather than just the trivial one.

REMARK 3.7 (The case of $E$ topologically non-trivial). Firstly, observe that the factorisation (3.2) and so Lemma 3.2 generalises to this case - the construction that is performed there is independent of the fact that $A_{n}=0$, by standard arguments of construction of global PDOs. So we obtain a first order PDO $B$ acting on sections and the local calculations in Lemma 3.2 (equations (3.6), 3.7), (3.8) ) carry over in the trivialisation where $A_{n}=0$. Therefore, by the proof of Proposition 3.3 , we have $\Lambda_{g, A, Q} \equiv-\left.B\right|_{\partial M}$ modulo smoothing.

Our main result of the chapter, Theorem 3.4, remains valid in the following form. By Lemma 2.4 we may assume that $(A-B)\left(\frac{\partial}{\partial x^{n}}\right)=0$ in a neighbourhood of the boundary. For a coordinate chart $W \subset \partial M$ and some given trivialisation of $\left.E\right|_{W}$, we may extend this trivialisation to a neighbourhood $W \times[0, \epsilon)$ of $W$ in $M$. Again, by the proof of Lemma 2.4 we may change the trivialisation by a gauge transformation such that $A_{n}=B_{n}=0$ locally. Then the extraction of the Taylor series from the full symbol of $B$ works the same as before and we have the full jet of $(A-B) \in \Omega^{1}(M ;$ End $E)$ vanishing at the boundary.

Remarks 3.5 and 3.6 clearly generalise to this setting. 


\section{CHAPTER 4}

\section{Gaussian Beams}

In this chapter, we will construct the Gaussian Beam quasimodes (or generalised approximate eigenfunctions) that concentrate near geodesics, for the purposes of constructing the CGO solutions in the case where the transversal manifold is not necessarily simple. Moreover, we will use the method described in $[\mathbf{2 3}$, where it was used in the case of a scalar potential and no first order term - here we also consider the vector case and a first order term. More precisely, we consider CGO solutions of the form $e^{-s x_{1}}\left(v_{s}+r_{s}\right)$ for the general operator $\Delta+X+q$, where $s=\tau+i \lambda$, with $\tau$ and $\lambda$ real; we want to guarantee certain behaviour of the solutions in the limit as $\tau \rightarrow \infty$. In Section 4.1 we construct the Gaussian Beams and in Section 4.2 we use them to construct the CGOs. We start by motivating our definition:

- Since $v_{s}$ is the main part of the solutions we would like to have $e^{s x_{1}}(\Delta+X+Q) e^{-s x_{1}} v_{s}$ small in $L^{2}$ norm.

- The solutions should concentrate along geodesics in a certain way.

- Simple manifold case: this is covered in Proposition 4.3 below and motivates the general transversal manifold case.

Throughout the chapter, we are working in the setting of $M \Subset\left(\mathbb{R} \times M_{0}, e \oplus g\right)$ with $\operatorname{dim} M_{0}+$ $1=\operatorname{dim} M=n \geq 3$.

DeFinition 4.1 (Generalised quasimodes). Given a family of functions $v_{s}$ on $M$ depending on a parameter $s=\tau+i \lambda(\tau, \lambda \in \mathbb{R})$, we say that $v_{s}$ is a generalised approximate eigenfunction or generalised quasimode if $\left\|v_{s}\right\|_{L^{2}(M)}=O(1)$ as $\tau \rightarrow \infty$ and:

$$
\begin{aligned}
\left\|\left(\left(\Delta_{g}+X+q\right)+s\left(2 \frac{\partial}{\partial x_{1}}-X_{1}\right)-s^{2}\right) v_{s}\right\|_{L^{2}(M)} & =\left\|e^{s x_{1}}(\Delta+X+q) e^{-s x_{1}} v_{s}\right\|_{L^{2}(M)} \\
& =o(|\tau|)
\end{aligned}
$$

REMARK 4.2. The main difference between this and the definition of a quasimode found in 23 is that the definition of a quasimode is independent of the $x_{1}$ direction, i.e. $v_{s}$ there was a function defined on $M_{0}$ only and it was only asked that $\left\|\left(\Delta-s^{2}\right) v_{s}\right\|_{L^{2}\left(M_{0}\right)}=o(|\tau|)$. This produces certain problems for us in the sense that the twisted Laplacian $d_{A}^{*} d_{A}$ now splits in a non-trivial way in an $x_{1}$ component, $x^{\prime}$ component and a mixed component, unlike the ordinary Laplacian, $\Delta_{e \oplus g}=-|g|^{-1 / 2} \frac{\partial}{\partial x_{1}}\left(|g|^{1 / 2} \frac{\partial}{\partial x_{1}}\right)+\Delta_{g}$. As we will shortly see, this amounts to solving a certain $\bar{\partial}$-equation, which complicates things.

\subsection{Main constructions of Gaussian Beams}

We will focus on constructing generalised quasimodes. A complex vector field $X$ on $M$ is a skew-Hermitian vector field if $X^{*}=-X$ in the complexified tangent bundle $T_{\mathbb{C}} M$; moreover, we have the notion of a skew-Hermitian matrix of vector fields, which is a clear generalisation of the previously defined term. As a warm up for the general construction, we will first deal with the easy case of line bundles and $M_{0}$ simple, which comes out of our work in Chapter 6 - in this case we have an ansatz for the eikonal equation. 
Recall also that a unit speed geodesic $\gamma:[0, L] \rightarrow M$ is called non-tangential if $\gamma(0), \gamma(L) \in$ $\partial M$ and $\dot{\gamma}(0), \dot{\gamma}(L)$ are not parallel to $\partial M$, with $\gamma(t)$ in the interior of $M$ for $0<t<L$.

Proposition 4.3. Let $\left(M_{0}, g\right)$ be a simple manifold and $\gamma:[0, L] \rightarrow M_{0}$ a non-tangential geodesic and let $\lambda$ be a real parameter. Let $X$ and $Y$ be two smooth skew-Hermitian vector fields on $M$. Then there exists a family of generalised quasimodes satisfying the above conditions, i.e. if $s=\tau+i \lambda$, then there exists $v_{s}, \omega_{s} \in C^{\infty}\left(\mathbb{R} \times M_{0}\right)$ such that:

$$
\begin{aligned}
& \left\|\left(\left(\Delta_{g}+X+q\right)+s\left(2 \frac{\partial}{\partial x_{1}}-X_{1}\right)-s^{2}\right) v_{s}\right\|_{L^{2}(M)}=o(|\tau|) \quad \text { and } \quad\left\|v_{s}\right\|_{L^{2}(M)}=O(1) \\
& \left\|\left(\left(\Delta_{g}+Y+q\right)-s\left(2 \frac{\partial}{\partial x_{1}}-Y_{1}\right)-s^{2}\right) \omega_{s}\right\|_{L^{2}(M)}=o(|\tau|) \quad \text { and } \quad\left\|\omega_{s}\right\|_{L^{2}(M)}=O(1)
\end{aligned}
$$

as $\tau \rightarrow \infty$ and for each $\phi \in C\left(M_{0}\right)$ and $x_{1}^{\prime} \in \mathbb{R}$ we have:

$$
\lim _{\tau \rightarrow \infty} \int_{\left\{x_{1}^{\prime}\right\} \times M_{0}} v_{s} \bar{\omega}_{s} \phi d V_{g}=\int_{0}^{L} e^{2 \lambda t} e^{\Phi_{1}+\bar{\Phi}_{2}} \phi(\gamma(t)) d t
$$

where $\Phi_{1}$ and $\Phi_{2}$ are smooth on $\mathbb{R} \times[0, L]$ and satisfy the following equations 1 ,

$$
\left(\frac{\partial}{\partial x_{1}}+i \frac{\partial}{\partial r}\right)\left(\Phi_{1}\right)=\frac{1}{2}\left(X_{1}+i X_{r}\right) \quad \text { and } \quad\left(-\frac{\partial}{\partial x_{1}}+i \frac{\partial}{\partial r}\right)\left(\Phi_{2}\right)=\frac{1}{2}\left(-Y_{1}+i Y_{r}\right)
$$

Proof. As in Section 6.1.1, consider a simple manifold $D$ which contains $M_{0}$ and a point $p \in D$ such that $\mathbb{R} \times\{p\}$ is disjoint from $M$ and consider the global polar coordinate system at this point. Furthermore, we proceed by picking a different conjugating exponent - we let $\rho=x_{1}+i r$. By Lemma 6.1.

$$
e^{s \rho}(\Delta+X+q) e^{-s \rho} u_{s}=(\Delta+X+q) u_{s}-s(\Delta \rho+X(\rho)-2\langle d \rho, d \cdot\rangle) u_{s}-s^{2}|d \rho|^{2} u_{s}
$$

One wants to have a handle on the size of the right hand side, so one equates the linear and the quadratic terms in $s$ to zero; this is done in Chapter 6. The same construction gives us $u_{s}=|g|^{-1 / 4} \cdot a \cdot b_{\tau}(\theta)$, where $a$ and $b_{\tau} \in C^{\infty}\left(S^{n-2}\right)$ are chosen such that:

$$
\begin{gathered}
\left(\frac{\partial}{\partial x_{1}}+i \frac{\partial}{\partial r}\right)(a)=\frac{1}{2}\left(X_{1}+i X_{r}\right) a \\
\left\|b_{\tau}\right\|_{L^{2}\left(S^{n-2}\right)}^{2}=1, \quad\left\|b_{\tau}\right\|_{W^{2, \infty}\left(S^{n-2}\right)}^{2}=O\left(\tau^{\alpha}\right) \quad \text { and } \quad\left|b_{\tau}\right|^{2} d S \rightarrow \delta_{\theta_{0}}
\end{gathered}
$$

i.e. $b_{\tau}$ is a $C^{\infty}$ approximation to the delta function, with $\alpha<1$; here $d S$ is the volume element of $S^{n-2}$. We pick $a$ of the form $e^{\Phi_{1}}$, so that $\Phi_{1}$ satisfies the equation:

$$
\left(\frac{\partial}{\partial x_{1}}+i \frac{\partial}{\partial r}\right)\left(\Phi_{1}\right)=\frac{1}{2}\left(X_{1}+i X_{r}\right)
$$

Now, given $u_{s}$ as above, we set $v_{s}=e^{-i s r} u_{s}$ :

$$
\begin{aligned}
e^{s x_{1}}(\Delta+X+q) e^{-s x_{1}} v_{s} & =e^{-i s r} e^{s \rho}(\Delta+X+q) e^{-s \rho} u_{s} \\
& =e^{-i s r}(\Delta+X+q)\left(|g|^{-1 / 4} \cdot a \cdot b_{\tau}(\theta)\right)=: f
\end{aligned}
$$

By using the properties of $b_{\tau}$ and the boundedness of other factors, we see that $f$ is clearly equal to $O\left(\tau^{\alpha}\right)$ in $L^{2}(M)$ with $\alpha<1$. But this exactly means that $v_{s}$ is a generalised approximate eigenfunction. Analogously we construct the $\omega_{s}$ function with respect to $Y$, but with one difference in mind - we take $-x_{1}$ to be the Carleman weight (this will be important in the integral

\footnotetext{
${ }^{1}$ In these equations, we extend the domain of definition of $X$ and $Y$ from $M$ to $\mathbb{R} \times M_{0}$ smoothly to compactly supported vector fields and with a slight abuse of notation still denote them the same.
} 
identity). Moreover, we have:

$$
\int_{\left\{x_{1}^{\prime}\right\} \times M_{0}} v_{s} \bar{\omega}_{s} \phi d V_{g} \rightarrow \int_{0}^{L} e^{2 \lambda r} e^{\Phi_{1}+\bar{\Phi}_{2}} \phi(\gamma(r)) d r
$$

when $\tau \rightarrow \infty$, for each $x_{1}^{\prime}$, by using that the volume element on $M_{0}$ is $d V_{g_{0}}=|g|^{\frac{1}{2}} d x_{2} \wedge \ldots \wedge d x_{n}$ and the concentration properties of $b_{\tau}$.

Now we are ready to make the passage to the case of the transversal manifold being nonsimple, with the previous proposition giving us some intuition. Most of the proof we are about to see is analogous to the proof of Proposition 3.1 in $[\mathbf{2 3}]$. The main difference is that, when constructing the amplitude $a$ in $v_{s}=e^{i s \Theta} a$, we do not get an ordinary differential equation we get that $a$ satisfies a certain $\bar{\partial}$-equation. This complicates the construction of $a$ slightly and uses the properties of $\bar{\partial}$-equations we discuss in Subsection 6.1.3. Moreover, the derivation of the limit integral is also more involved. We will prove the following theorem for line bundles first and then generalise to all vector bundles in a series of results after.

Theorem 4.4 (Main construction of the Gaussian Beams). Let $\gamma:[0, L] \rightarrow M_{0}$ be a nontangential geodesic and let $\lambda$ be a real parameter, with $M_{0}$ any compact manifold with boundary. Let $X$ and $Y$ be two smooth skew-Hermitian vector fields on $M$, which we extend to compactly supported vector fields on $\mathbb{R} \times M_{0}$ (still denoted $X$ and $Y$ ). Then there exists a family of generalised quasimodes satisfying the above conditions, i.e. if $s=\tau+i \lambda$, then there exists $v_{s}, \omega_{s} \in C^{\infty}\left(J_{0} \times M_{0}\right)$, where $J_{0}=\left[-N_{0}, N_{0}\right]$ for some large positive integer $N_{0}$, such that:

$$
\begin{aligned}
& \left\|\left(\left(\Delta_{g}+X+q\right)+s\left(2 \frac{\partial}{\partial x_{1}}-X_{1}\right)-s^{2}\right) v_{s}\right\|_{L^{2}\left(J_{0} \times M_{0}\right)}=o(|\tau|) \quad \text { and } \quad\left\|v_{s}\right\|_{L^{2}\left(J_{0} \times M_{0}\right)}=O(1) \\
& \left\|\left(\left(\Delta_{g}+Y+q\right)-s\left(2 \frac{\partial}{\partial x_{1}}-Y_{1}\right)-s^{2}\right) \omega_{s}\right\|_{L^{2}\left(J_{0} \times M_{0}\right)}=o(|\tau|) \text { and }\left\|\omega_{s}\right\|_{L^{2}\left(J_{0} \times M_{0}\right)}=O(1)
\end{aligned}
$$

as $\tau \rightarrow \infty$ and for each $\phi \in C\left(M_{0}\right)$ and $x_{1}^{\prime} \in\left[-N_{0}, N_{0}\right]$ we have:

$$
\lim _{\tau \rightarrow \infty} \int_{\left\{x_{1}^{\prime}\right\} \times M_{0}} v_{s} \bar{\omega}_{s} \phi d V_{g}=\int_{0}^{L} e^{-2 \lambda t} e^{\Phi_{1}+\bar{\Phi}_{2}} \phi(\gamma(t)) d t
$$

where $\Phi_{1}$ and $\Phi_{2}$ are smooth on $\mathbb{R} \times[0, L]$ and satisfy the following equations:

$$
\left(\frac{\partial}{\partial x_{1}}-i \frac{\partial}{\partial r}\right)\left(\Phi_{1}\right)=\frac{1}{2}\left(X_{1}-i X_{r}\right) \quad \text { and } \quad\left(\frac{\partial}{\partial x_{1}}-i \frac{\partial}{\partial r}\right)\left(\bar{\Phi}_{2}\right)=\frac{1}{2}\left(-Y_{1}+i Y_{r}\right)
$$

Moreover, the following limit holds for $v_{s}$ and $\omega_{s}$ and any one form $\alpha$ on $M_{0}$ :

$$
\begin{aligned}
\lim _{\tau \rightarrow \infty} \frac{1}{\tau} \int_{\left\{x_{1}^{\prime}\right\} \times M_{0}}\left\langle\alpha, d v_{s}\right\rangle \bar{\omega}_{s} \phi d V_{g} & =\int_{0}^{L} i \alpha(\dot{\gamma}(t)) e^{\Phi_{1}+\bar{\Phi}_{2}} e^{-2 \lambda t} \phi(\gamma(t)) d t \\
\lim _{\tau \rightarrow \infty} \frac{1}{\tau} \int_{\left\{x_{1}^{\prime}\right\} \times M_{0}}\left\langle\alpha, d \bar{\omega}_{s}\right\rangle v_{s} \phi d V_{g} & =-\int_{0}^{L} i \alpha(\dot{\gamma}(t)) e^{\Phi_{1}+\bar{\Phi}_{2}} e^{-2 \lambda t} \phi(\gamma(t)) d t
\end{aligned}
$$

Proof. Firstly, let us isometrically embed our manifold $\left(M_{0}, g\right)$ into a larger closed manifold $(\widehat{M}, g)$ of the same dimension. This is possible since we can form the manifold $\widehat{M}=M_{0} \sqcup_{\partial M_{0}} M_{0}$, which is the disjoint union of two copies of $M_{0}$, glued along the boundary; $g, X$ and $Y$ are smoothly extended to $\mathbb{R} \times \widehat{M}$. We will extend the geodesic such that for $\epsilon>0$ we have $\gamma(t) \epsilon$ $\hat{M} \backslash M_{0}$ for $t \in(-2 \epsilon, 0) \cup(L, L+2 \epsilon)$; this is possible since $\gamma$ is non-tangential. Let $N_{0}$ be a large positive integer such that $\left(-N_{0}, N_{0}\right) \times M_{0}$ contains $M$ and the support of $X$ and $Y$; let us introduce the notation for the interval $J_{1}:=\left[-N_{0}-1, N_{0}+1\right]$.

Let us first introduce a set of local coordinates along the geodesic; a detailed account of this can be found in 23 . Since our manifold is compact and $\gamma$ has no loops, we can assume 


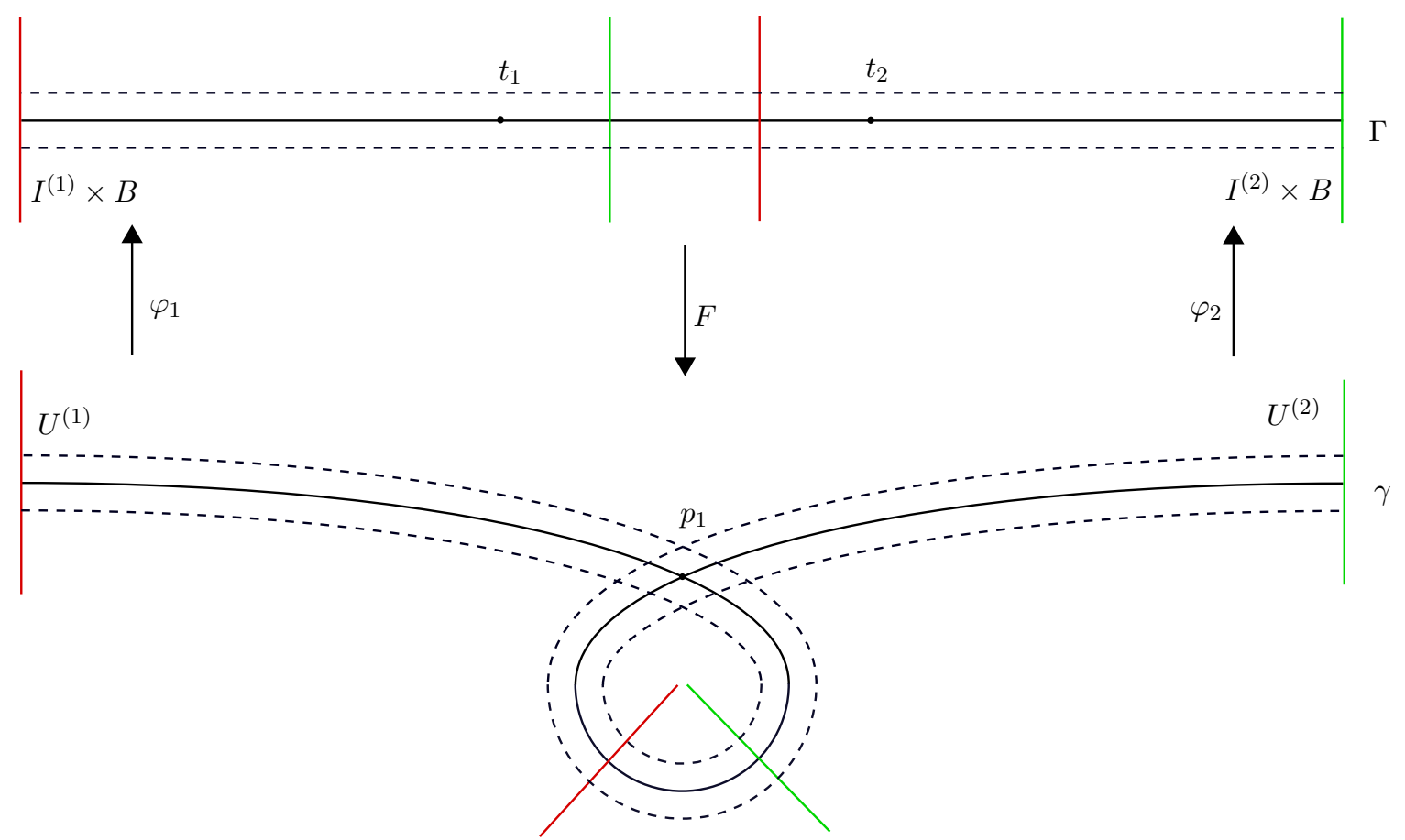

Figure 1. An illustration of the local diffeomorphism $F$ obtained from Fermi coordinates: the cover of the geodesic $\gamma$ is given by charts $\left(U^{(1)}, \varphi_{1}\right)$ and $\left(U^{(2)}, \varphi_{2}\right)$, with $\varphi_{i}\left(U^{(i)}\right)=I^{(i)} \times B$ for $i=1,2$. Red colour delimits the $U^{(1)}$ piece, the green one delimits $U^{(2)}$ and $\gamma\left(t_{1}\right)=\gamma\left(t_{2}\right)=p_{1}$.

$\gamma$ self-intersects only finitely many times, at $0<t_{1}<\ldots<t_{N^{\prime}}<L$ and that there is an open cover $\left\{\left(U^{(j)}, \varphi_{j}\right)\right\}_{j=0}^{N^{\prime}+1}$ of $\gamma([-\epsilon, L+\epsilon])$ such that $\varphi_{j}\left(U^{(j)}\right)=I^{(j)} \times B$, where $I^{(j)}$ s are open intervals and $B$ a small $n-2$-dimensional ball. Also, $\varphi_{j}(\gamma(t))=(t, 0)$ and $t_{j}$ s belong only to $I^{(j)}$ S and $\bar{I}^{(j)} \cap \bar{I}^{(k)}=\emptyset$ unless $|j-k| \leq 1 ; \varphi_{i}$ s agree on overlaps. These are called the Fermi coordinates and they have the following two properties along the geodesic: the metric is diagonal and $\partial_{i} g^{j k}=0$ (and so the Christoffel symbols vanish). Also, let us denote by $F$ the map from $U=[-2 \epsilon, L+2 \epsilon] \times B$ to $\widehat{M}$, which restricts to the inverse charts on $I^{(i)} \times B \mathrm{~s}$; this is well defined since the charts agree on overlaps. The map $F$ is locally a diffeomorphism, but is not globally because of self-intersections of the geodesic (see Figure 1).

Rather than constructing the quasimode locally, near a point $p_{0}=\gamma\left(t_{0}\right)$ on $\gamma([-\epsilon, L+\epsilon])$, observe that we may use the map $F$ as a local diffeomorphism and pull back all the data $(g$, $X$ and $Y$ ) to $\mathbb{R} \times U$ - let us still denote the pullbacks with the same letters. Let us also use the notation $D_{i}:=J_{i} \times U$ for $i=0$ and 1 . We will use the coordinate $y$ on $B$ and denote the geodesic in these local coordinates as $\Gamma=\{(t, 0)\}$ in $U$. Furthermore, we will construct the quasimode on $D$ and then provide a method to pushforward this quasimode to $J_{0} \times M_{0}$.

Let us seek for solutions of the form $v_{s}=e^{i s \Theta} a$, where $a$ and $\Theta$ will be complex functions supported in $|y|<\delta^{\prime} / 2$. Then we have:

$$
\begin{aligned}
& e^{s x_{1}}(\Delta+X+q) e^{-s x_{1}} v_{s}=e^{i s \Theta} e^{-s\left(-x_{1}+i \Theta\right)}(\Delta+X+q) e^{s\left(-x_{1}+i \Theta\right)} a \\
= & e^{i s \Theta}\left\{(\Delta+X+q) a+s\left(2 \frac{\partial a}{\partial x_{1}}-2 i\langle d \Theta, d a\rangle+\left(-X_{1}+i X(\Theta)\right) a+i \Delta(\Theta) a\right)-s^{2}\left(1-|d \Theta|^{2}\right) a\right\}
\end{aligned}
$$


by putting $\rho=-x_{1}+i \Theta$ and using Lemma 6.1. Firstly, let us solve $|d \Theta|^{2}=1$ up to order $|y|^{N}$ on $\Gamma$. We look for $\Theta$ in the form $\Theta=\sum_{j=0}^{N} \Theta_{j}$, where:

$$
\Theta_{j}=\sum_{|\alpha|=j} \frac{\Theta_{j, \alpha}(t)}{\alpha !} y^{\alpha}
$$

are the homogeneous components and we write $g^{j k}=\sum_{l=0}^{N} g_{l}^{j k}+r_{N+1}^{j k}$, where

$$
g_{l}^{j k}(t, y)=\sum_{|\beta|=l} \frac{g_{l, \beta}^{j k}(t)}{\beta !} y^{\beta} \quad \text { and } \quad r_{N+1}^{j k}=O\left(|y|^{N+1}\right)
$$

is the remainder in Taylor's theorem. By the properties of the coordinates, we have $g_{0}^{j k}=\delta^{j k}$ and $g_{1}^{j k}=0$. Let us accordingly choose $\Theta_{0}(t, y)=t$ and $\Theta_{1}(t, y)=0$. Most of the next step follows from the lines of $[\mathbf{2 3}$, but we give it here for completeness:

$$
\begin{gathered}
\langle d \Theta, d \Theta\rangle-1=g^{j k} \partial_{j} \Theta \partial_{k} \Theta-1=\left(1+g_{2}^{11}+\ldots\right)\left(1+\partial_{t} \Theta_{2}+\ldots\right)\left(1+\partial_{t} \Theta_{2}+\ldots\right) \\
+2\left(g_{2}^{1 \alpha}+\ldots\right)\left(1+\partial_{t} \Theta_{2}+\ldots\right)\left(\partial_{y_{\alpha}} \Theta_{2}+\ldots\right) \\
+\left(\delta^{\alpha \beta}+g_{2}^{\alpha \beta}+\ldots\right)\left(\partial_{y_{\alpha}} \Theta_{2}+\partial_{y_{\alpha}} \Theta_{3}+\ldots\right)\left(\partial_{y_{\beta}} \Theta_{2}+\partial_{y_{\beta}} \Theta_{3}+\ldots\right)-1 \\
=\left[2 \partial_{t} \Theta_{2}+\nabla_{y} \Theta_{2} \cdot \nabla_{y} \Theta_{2}+g_{2}^{11}\right]+\sum_{p=2}^{N}(\ldots)+O\left(|y|^{N+1}\right)
\end{gathered}
$$

We want to choose $\Theta_{i}$ such that the first bracket and the sum above vanish. We pick $\Theta_{2}(t, y)=$ $\frac{1}{2} H(t) y \cdot y$ where $H(t)$ is a smooth complex symmetric matrix. For the first bracket to vanish, we need to have:

$$
\dot{H}(t)+H(t)^{2}=F(t)
$$

where $F(t)$ is the symmetric matrix determined by $g_{2}^{11}(t, y)=-F(t) y \cdot y$. Choosing $H_{0}=H\left(t_{0}\right)$ for $t_{0}:=-2 \epsilon$ to be any complex symmetric matrix with $\operatorname{Im}(H)$ positive definite; following [23 this Riccati equation has a unique smooth complex symmetric solution $H(t)$ with $\operatorname{Im}(H(t))$ positive definite for all $t \in[-2 \epsilon, L+2 \epsilon]$. Now we find $\Theta_{3}, \ldots, \Theta_{N}$ by inductively solving the first order ODEs along $\Gamma$ with an initial condition at $t_{0}$, obtained by collecting the homogeneous terms in $y$ of higher order in the previous expansion. We obtain a smooth $\Theta$ such that $|d \Theta|^{2}=1$ up to order $|y|^{N}$.

Now we turn to the more interesting step, how to solve:

$$
s\left[2 \frac{\partial a}{\partial x_{1}}-2 i\langle d \Theta, d a\rangle+\left(-X_{1}+i X(\Theta)\right) a+i \Delta(\Theta) a\right]+(\Delta+X) a=0
$$

up to order $|y|^{N}$. We look for $a$ in the form

$$
a=\tau^{\frac{n-2}{4}}\left(a_{0}+s^{-1} a_{-1}+\cdots+s^{-N} a_{-N}\right) \chi\left(\frac{y}{\delta^{\prime}}\right)
$$

where $\chi$ is a bump function defined such that $\chi=1$ on $|y| \leq 1 / 4, \chi=0$ for $|y| \geq 1 / 2$. We now equate each degree of $s$ in the above expression to zero and obtain $N+1$ equations for each degree $1,0, \ldots,-(N-1)$ :

$$
\begin{gathered}
2 \frac{\partial a_{0}}{\partial x_{1}}-2 i\left\langle d \Theta, d a_{0}\right\rangle+\left(-X_{1}+i X(\Theta)+i \Delta(\Theta)\right) a_{0}=0 \\
2 \frac{\partial a_{j}}{\partial x_{1}}-2 i\left\langle d \Theta, d a_{j}\right\rangle+\left(-X_{1}+i X(\Theta)+i \Delta(\Theta)\right) a_{j}+(\Delta+X) a_{j+1}=0
\end{gathered}
$$

for each $j=-1, \ldots,-N$. Let us introduce $\eta=i \Delta \Theta-X_{1}+i X(\Theta)$ and write $\eta=\eta_{0}+\ldots+$ $\eta_{N}+O\left(|y|^{N+1}\right)$ for the Taylor expansion of $\eta$. We look for $a_{0}=a_{00}+a_{01}+\ldots+a_{0 N}$ where 
each $a_{0 i}$ is a homogeneous polynomial of degree $i$. Then the degree one equation becomes:

$$
2 \frac{\partial}{\partial x_{1}}\left(a_{00}+\ldots+a_{0 N}\right)-2 i g^{j k} \partial_{j} \Theta \partial_{k} a+\left(\eta_{0}+\eta_{1}+\ldots\right)\left(a_{00}+\ldots+a_{0 N}\right)=0
$$

to order $|y|^{N}$. After rewriting, this becomes:

$$
\begin{aligned}
&=2 \frac{\partial}{\partial x_{1}}\left(a_{00}+\ldots a_{0 N}\right)-2 i\left(1+g_{2}^{11}+\ldots\right)\left(1+\partial_{t} \Theta_{2}+\ldots\right)\left(\partial_{t} a_{00}+\partial_{t} a_{01}+\ldots\right) \\
&- 2 i\left(g_{2}^{1 \beta}+\ldots\right)\left(1+\partial_{t} \Theta_{2}+\ldots\right)\left(\partial_{y_{\beta}} a_{01}+\ldots\right) \\
&- 2 i\left(g_{2}^{\alpha 1}+\ldots\right)\left(\partial_{y_{\alpha}} \Theta_{2}+\ldots\right)\left(\partial_{t} a_{00}+\partial_{t} a_{01}+\ldots\right) \\
&- 2 i\left(\delta^{\alpha \beta}+g_{2}^{\alpha \beta}+\ldots\right)\left(\partial_{y_{\alpha}} \Theta_{2}+\ldots\right)\left(\partial_{y_{\beta}} a_{01}+\ldots\right) \\
&+\left(\eta_{0}+\eta_{1}+\ldots+\eta_{N}+O\left(|y|^{N+1}\right)\right)\left(a_{00}+a_{01}+\ldots+a_{0 N}\right) \\
&=\left[2 \frac{\partial a_{00}}{\partial x_{1}}-2 i \partial_{t} a_{00}+\eta_{0} a_{00}\right]+\left[2 \frac{\partial a_{01}}{\partial x_{1}}-2 i \partial_{t} a_{01}-2 i \partial_{y_{\alpha}} \Theta_{2} \partial_{y_{\alpha}} a_{01}+\eta_{1} a_{00}+\eta_{0} a_{01}\right]+\ldots
\end{aligned}
$$

where we have written down the first two terms in the $y$ expansion. For us, the equation for $a_{00}$ is particularly important (it will give us the value of $a_{0}$ along $\Gamma$ ). We have that $\eta_{0}=$ $\left(i \Delta \Theta-X_{1}+i X(\Theta)\right)(t, 0)$, where we know that $\Theta=t+1 / 2 H(t) y \cdot y+O\left(|y|^{3}\right)$. Therefore, we compute:

$$
\Delta \Theta(t, 0)=-|g|^{-\frac{1}{2}} \frac{\partial}{\partial x^{j}}\left(|g|^{\frac{1}{2}} g^{j k} \frac{\partial \Theta}{\partial x^{k}}\right)=-|g|^{-\frac{1}{2}} \frac{\partial|g|^{\frac{1}{2}}}{\partial t}-\delta^{j k} H_{j k}=-\operatorname{tr} H(t)
$$

So, our equation for $a_{00}$ becomes:

$$
\left(\frac{\partial}{\partial x_{1}}-i \frac{\partial}{\partial t}\right) a_{00}=\frac{1}{2}\left(X_{1}-i X_{t}+i \operatorname{tr} H(t)\right) a_{00}
$$

which we have seen in a more general, matrix case. Here, we want a solution of the form $a_{00}=e^{\Phi_{1}+f_{1}}$, so that we obtain, for $\partial=1 / 2\left(\partial / \partial x_{1}-i \partial / \partial t\right)$

$$
\partial \Phi_{1}=\frac{1}{4}\left(X_{1}-i X_{t}\right) \text { and } \quad \frac{\partial f_{1}}{\partial t}=-\frac{1}{2} \operatorname{tr} H(t)
$$

where $\Phi_{1}$ is a function in both $x_{1}$ and $t, f_{1}$ is a function of just $t$. Now for the rest of the $a_{0 i}$ for $i>0$, we obtain a similar vector valued equation of the form:

$$
\partial v+A v+f=0
$$

where $v$ and $f$ are vectors and $A$ is a matrix. The reason for this is that for $i>0$, we get more components in the Taylor expansion, so we get a coefficient for each (think of $a_{0 i} \mathrm{~s}$ as vectors). This is solvable by our previous work on fundamental solutions of such equations, so that we produce an invertible matrix $C$ such that

$$
\partial C=-A C
$$

in $\mathbb{R} \times(-2 \epsilon, L+2 \epsilon)$ (see Section 6.1.3). Then we try $v=C u$ for some vector function $u$ and we get the equation: $\partial u=-C^{-1} f$, which we know how to solve in the bounded domain $J_{0} \times\left[-\frac{3}{2} \epsilon, L+\frac{3}{2} \epsilon\right]$, by e.g. multiplying $f$ with a cut-off function, equal to one on $J_{0} \times\left[-\frac{3}{2} \epsilon, L+\frac{3}{2} \epsilon\right]$ and supported in $J_{1} \times(-2 \epsilon, L+2 \epsilon)$ in order to extend it to the whole $\left(x_{1}, t\right)$-plane and use the generalised Cauchy integral formula. Hence we determine $a_{0}$ and proceed to determine $a_{i}$ s for $i>0$ inductively. Notice also that $X$ is compactly supported, so we may indeed take the zero extension of it to the $\left(x_{1}, t\right)$-plane and solve the first equation in 4.3 .

At this point we make a remark about constructing the $\omega_{s}$ solution, which is the solution where we use $e^{s x_{1}}$ exponent in the CGO solution (and hence the $-s$ in the formulation of the 
theorem). The point is that everything just gets a minus sign at each spot where we use $x_{1}$. Checking through the details, we obtain a version of the equation 4.3) (we use the fact that $Y$ is skew-Hermitian):

$$
\partial \bar{\Phi}_{2}=\frac{1}{4}\left(-Y_{1}+i Y_{t}\right) \text { and } \quad \frac{\partial f_{2}}{\partial t}=-\frac{1}{2} \operatorname{tr} H(t)
$$

We are left with the terms of the form:

$$
\begin{aligned}
e^{s x_{1}}(\Delta+X) e^{-s x_{1}} e^{i s \Theta} a=e^{i s \Theta} \tau^{\frac{n-2}{4}}\left[s^{2} h_{2} a+\right. & s h_{1}+\cdots+s^{-(N-1)} h^{-(N-1)} \\
& \left.+s^{-N}(\Delta+X) a_{-N}\right] \chi\left(\frac{y}{\delta^{\prime}}\right)+e^{i s \Theta} \tau^{\frac{n-2}{4}} s b \tilde{\chi}\left(\frac{y}{\delta^{\prime}}\right)
\end{aligned}
$$

where we have $h_{j}$ s to be equal to zero to order $|y|^{N}$ on $\Gamma$; we also introduce $b$ and $\tilde{\chi}$ to describe the leftover terms which appear upon differentiating the function $\chi$ in a sum, but which therefore are zero near and far away of $\Gamma$. Concretely, we have $b=0$ for $|y| \leq \delta^{\prime} / 4$ and $\tilde{\chi}=0$ for $|y| \geq 1 / 2$ and the most important fact about this term is that it is linear in $s$.

In order to determine some bounds on $v_{s}$, let us introduce a positive constant $c$, for which it holds that $\operatorname{Im} H(t) y \cdot y \geq c|y|^{2}$. Then we have:

$$
\begin{aligned}
\left|e^{i s \Theta}\right| & =e^{-\lambda \operatorname{Re} \Theta} e^{-\tau \operatorname{Im} \Theta}=e^{-\lambda t} e^{-\lambda O\left(|y|^{2}\right)} e^{-\frac{\tau}{2} \operatorname{Im} H(t) y \cdot y} e^{-\tau O\left(|y|^{3}\right)} \\
\left|v_{s}\left(x_{1}, t, y\right)\right| & \lesssim \tau^{\frac{n-2}{4}} e^{-\frac{1}{4} c \tau|y|^{2}} \chi\left(\frac{y}{\delta^{\prime}}\right)
\end{aligned}
$$

after decreasing $\delta^{\prime}$ if necessary and using the $1 / 4$ factor in the exponential to dominate the remaining $O\left(|y|^{3}\right)$ factor, for $x_{1} \in J_{0}$. Thus we have:

$$
\begin{gathered}
\left\|v_{s}\right\|_{L^{2}\left(J_{0} \times U\right)} \lesssim\left\|\tau^{\frac{n-2}{4}} e^{-\frac{1}{4} c \tau|y|^{2}}\right\|_{L^{2}\left(J_{0} \times U\right)}=O(1) \\
\left\|e^{s x_{1}}(\Delta+X) e^{-s x_{1}} v_{s}\right\|_{L^{2}\left(J_{0} \times U\right)} \lesssim\left\|\tau^{\frac{n-2}{4}} e^{-\frac{1}{4} c \tau|y|^{2}\left(\tau^{2}|y|^{N+1}+\tau^{-N}+\tau b \tilde{\chi}\right)}\right\|_{L^{2}\left(J_{0} \times U\right)}=O\left(|\tau|^{\frac{3-N}{2}}\right)
\end{gathered}
$$

where the second line is equal to $O\left(|\tau|^{-K}\right)$ upon setting $N=2 K+3$, for any fixed $K$, a positive integer.

Let us now record a boundary estimate for future purposes. Namely, since the geodesic intersects the boundary $\partial M_{0}$ transversely at $t=0$ and $t=L$, we can introduce the implicit coordinates $\left\{(t(y), y):|y|<\epsilon^{\prime}\right\}$ for some smooth function $t(y)$ and small $\epsilon^{\prime}>0$. Then for $\delta^{\prime}$ small enough:

$$
\left\|v_{s}\left(x_{1}, \cdot\right)\right\|_{L^{2}\left(\partial M_{0} \cap U\right)}^{2}=\int_{|y|<\epsilon^{\prime}}\left|v_{s}\left(x_{1}, t(y), y\right)\right|^{2} d S(y) \lesssim \int_{\mathbb{R}^{n-2}} \tau^{\frac{n-2}{2}} e^{-\frac{1}{2} c \tau|y|^{2}} d y=O(1)
$$

for $x_{1}$ in $J_{0}$ and as $|\tau| \rightarrow \infty$.

Now we are done with the local construction and bounds on $J_{0} \times[-\epsilon, L+\epsilon] \times B$ and want to glue the solutions together with desired concentration properties. Let us denote by $v_{s}^{(j)}$ the pushforward by the coordinate map $I d \times \varphi_{j}^{-1}$ of the so obtained solution on $J_{0} \times U^{(j)}$ (where $I d: \mathbb{R} \rightarrow \mathbb{R}$ is the identity map). We thus obtain $v_{s}^{(0)}, v_{s}^{(1)}, \ldots, v_{s}^{(r)}$. To glue these, let $\chi_{j}(t)$ be a partition of unity subordinate to $I^{(j)}$; the we extend these to $U^{(j)}$ by saying $\tilde{\chi}_{j}\left(x_{1}, t, y\right)=\chi_{j}(t)$ and finally let:

$$
v_{s}:=\sum_{j=0}^{r} \tilde{\chi}_{j} v_{s}^{(j)}
$$


The previous remark allows us to have $v_{s}^{(j)}=v_{s}^{(j+1)}$ in the overlaps $J_{0} \times\left(U^{(j)} \cap U^{(j+1)}\right)$. Now, pick small neighbourhoods of the geodesic self-intersection points $p_{1}, \ldots, p_{R}$ and call them $V_{1}, \ldots, V_{R}$; for $\delta^{\prime}$ sufficiently small, we get that $F$ is injective on the complement of the inverse image by $F$ of the $V_{i}$ s (see Figure 1). Therefore, we can pick a finite cover by $W_{1}, \ldots, W_{S}$ of the remaining points on the geodesic such that $W_{i} \subset U^{\left(l_{i}\right)}$ for some $l_{i}$ and $\operatorname{supp}\left(v_{s}\right) \subset\left(\cup V_{i}\right) \cup\left(\cup W_{j}\right)$ and moreover, the restrictions to these satisfy:

$$
\left.v_{s}\right|_{V_{i}}=\sum_{\gamma\left(t_{l}\right)=p_{i}} v_{s}^{(l)} \text { and }\left.\quad v_{s}\right|_{W_{i}}=v_{s}^{\left(l_{i}\right)}
$$

It is now clear that the wanted $L^{2}$ bounds on $v_{s}$ follow from our previous local considerations on each of $v_{s}^{(i)}$. We are left with the concentration results to prove - by considering the partitions of unity subordinate to $V_{i}$ s and $W_{j}$ s, we can assume that $\phi$ has compact support in one of these sets. Let us first consider the easier case where $\operatorname{supp}(\phi) \subset W_{k}$ for some $k$. By a completely analogous construction, we may assume that we have $\omega_{s}=e^{i s \Theta} b$ on $J_{0} \times[-\epsilon, L+\epsilon] \times B$, constructed with respect to $Y$ - notice that $\Theta$ is solved for independently of the vector fields $X$ and $Y$ (recall that we only want $|d \Theta|^{2}=1$ up to order $\left.|y|^{N}\right)$.

In $W_{k}$, we have $v_{s}=e^{i s \Theta} a$ and $\omega_{s}=e^{i s \Theta} b$, where we dropped the indices to simplify notation. Then we have:

$$
\begin{gathered}
\int_{\left\{x_{1}^{\prime}\right\} \times M_{0}} v_{s} \bar{\omega}_{s} \phi d V_{g}=\int e^{i s \Theta} e^{-i \bar{s} \Theta} a \bar{b} \phi d V_{g} \\
=\int_{0}^{L} \int_{\mathbb{R}^{n-2}} e^{-2 \lambda \operatorname{Re} \Theta} e^{-2 \tau \operatorname{Im} \Theta} \tau^{\frac{n-2}{2}}\left(a_{0}+O\left(\tau^{-1}\right)\right)\left(\bar{b}_{0}+O\left(\tau^{-1}\right)\right) \chi\left(y / \delta^{\prime}\right)^{2} \phi|g|^{1 / 2} d y d t \\
=\int_{0}^{L} \int_{\mathbb{R}^{n-2}} e^{-\operatorname{Im} H(t) y \cdot y} e^{-2 \tau^{-1 / 2} O\left(|x|^{3}\right)} e^{-2 \tau^{-1} O\left(|x|^{2}\right)} e^{-2 \lambda t} \tau^{\frac{n-2}{2}} \\
\cdot\left(a_{0}\left(t, \tau^{-1 / 2} x\right)+O\left(\tau^{-1}\right)\right)\left(\bar{b}_{0}\left(t, \tau^{-1 / 2} x\right)+O\left(\tau^{-1}\right)\right) \chi\left(x /\left(\tau^{1 / 2} \delta^{\prime}\right)\right)^{2}|g|^{1 / 2}\left(t, \tau^{-1 / 2} x\right) \phi d y d t
\end{gathered}
$$

by performing the substitution $y=\tau^{-1 / 2} x$; we can see what the limit is - namely, by bounding

$$
e^{-c|x|^{2}} e^{2 A|x|^{3} /\left(\tau^{1 / 2}\right)} e^{2 B|x|^{2} / \tau} \leq e^{|x|^{2}\left(-c+2 A \delta^{\prime}+2 B / \tau\right)}
$$

where $c$ is as before the positive constant such that $\operatorname{Im} H(t) y \cdot y \geq c|y|^{2}$ and using the fact that we integrate over $|y| \leq \tau^{1 / 2} \delta^{\prime}$, by taking sufficiently small $\delta^{\prime}$ we get exponent negative and hence we get an integrable function; thus we may use the Dominated convergence theorem to get this tends to, as $\tau \rightarrow \infty$ :

$$
\begin{aligned}
\int_{0}^{L} e^{-2 \lambda t} e^{\Phi_{1}+f_{1}+\bar{\Phi}_{2}+\bar{f}_{2}} \phi(\gamma(t)) \int_{\mathbb{R}^{n-2}} e^{-\operatorname{Im} H(t) x \cdot x} d x d t & \\
& =\int_{\mathbb{R}^{n-2}} e^{-|y|^{2}} d y \int_{0}^{L} \frac{e^{-2 \lambda t} e^{\Phi_{1}+f_{1}+\bar{\Phi}_{2}+\bar{f}_{2}} \phi(\gamma(t))}{\sqrt{\operatorname{det} \operatorname{Im} H(t)}} d t
\end{aligned}
$$

by using the linear change of variable by the matrix $\operatorname{Im} H(t)$. However, from before we know that:

$$
\operatorname{det}(\operatorname{Im} H(t))=\operatorname{det}\left(\operatorname{Im} H\left(t_{0}\right)\right) e^{-2 \int_{t_{0}}^{t} \operatorname{tr} \operatorname{Re} H(s) d s} \quad \text { and } \quad \frac{\partial\left(f_{1}+\bar{f}_{2}\right)}{\partial t}=-\operatorname{tr} \operatorname{Re} H(t)
$$

Hence we obtain cancellation in the above integral and by picking the initial condition for $H\left(t_{0}\right)$ such that $\operatorname{det}\left(\operatorname{Im} H\left(t_{0}\right)\right)=\pi^{n-2}$, we finally get the desired limit:

$$
\int_{0}^{L} e^{-2 \lambda t} e^{\Phi_{1}+\bar{\Phi}_{2}} \phi(\gamma(t)) d t
$$


Moreover, in the case where we have $\operatorname{supp}(\phi) \subset V_{j}$ for some $j$, we have $v_{s}=\sum_{\gamma\left(t_{l}\right)=p_{j}} v_{s}^{(l)}$ and $\omega_{s}=\sum_{\gamma\left(t_{l}\right)=p_{j}} \omega_{s}^{(l)}$, which means that we have the following expression:

$$
v_{s} \bar{\omega}_{s}=\sum_{\gamma\left(t_{l}\right)=p_{j}} v_{s}^{(l)} \bar{\omega}_{s}^{(l)}+\sum_{l \neq l^{\prime}, \gamma\left(t_{l}\right)=\gamma\left(t_{l^{\prime}}\right)=p_{j}} v_{s}^{(l)} \bar{\omega}_{s}^{\left(l^{\prime}\right)}
$$

We want to show that the mixed terms vanish; i.e. want to show $\int_{V_{j} \cap M_{0}} v_{s}^{(l)} \bar{\omega}_{s}^{\left(l^{\prime}\right)} \phi d V_{g} \rightarrow 0$ as $\tau \rightarrow \infty$ for $l \neq l^{\prime}$, so that we are left with the expression from the statement - this would prove our claim.

Let us use the fact that $\frac{\partial \Theta}{\partial t}(t, 0)=1$; write $v_{s}^{(l)}=e^{i s \Theta^{(l)}} a^{(l)}$ and $\omega_{s}^{(l)}=e^{i s \Theta^{(l)}} b^{(l)}$. This gives us that for $\varphi=\operatorname{Re}\left(\Theta^{(l)}-\Theta^{\left(l^{\prime}\right)}\right)$ we have $d \varphi \neq 0$ at the point $p_{j}$, as the geodesic intersects itself transversally. Therefore, by further reducing $\delta^{\prime}$ if necessary, we may assume that $d \varphi$ is non-vanishing in $V_{j}$. From now on, we drop the subscript $s$ to relax the notation.

Let $p^{(l)}=e^{-s \operatorname{Im} \Theta^{(l)}} e^{-\lambda \operatorname{Re} \Theta^{(l)}} a^{(l)}$ and analogously $q^{(l)}=e^{-s \operatorname{Im} \Theta^{(l)}} e^{-\lambda \operatorname{Re} \Theta^{(l)}} b^{(l)}$. Then we can write $v^{(l)}=e^{i \tau \operatorname{Re}\left(\Theta^{(l)}\right)} p^{(l)}$ and similarly $\omega^{(l)}=e^{i \tau \operatorname{Re}\left(\Theta^{(l)}\right)} q^{(l)}$. Then one can easily check that:

$$
\int_{V_{j} \cap M_{0}} v^{(l)} \bar{\omega}^{\left(l^{\prime}\right)} \phi d V_{g}=\int_{V_{j} \cap M_{0}} e^{i \tau \varphi} p^{(l)} \bar{q}^{\left(l^{\prime}\right)} \phi d V_{g}
$$

Fix $\epsilon^{\prime \prime}>0$. In order to be able to do calculus with $\phi$, we split it into a smooth and a sufficiently small part: let $\phi=\phi_{1}+\phi_{2}$, where $\phi_{1} \in C_{c}^{\infty}\left(V_{j} \cap M_{0}\right)$ smooth and $\left\|\phi_{2}\right\|_{L^{\infty}\left(V_{j} \cap M_{0}\right)} \leq \epsilon^{\prime \prime}$. For the $\phi_{2}$ part, we have the bound $\left|\int_{V_{j} \cap M_{0}} e^{i \tau \varphi} p^{(l)} \bar{q}^{\left(l^{\prime}\right)} \phi_{2} d V_{g}\right| \lesssim\left\|p^{(l)}\right\|_{L^{2}}\left\|\bar{q}^{\left(l^{\prime}\right)}\right\|_{L^{2}}\left\|\phi_{2}\right\|_{L^{\infty}} \lesssim \epsilon^{\prime \prime}$, since $\left\|p^{(l)}\right\|_{L^{2}} \lesssim\left\|v^{(l)}\right\|_{L^{2}}=O(1)$ and similarly for $q^{\left(l^{\prime}\right)}$.

For the main, smooth part we perform integration by parts with the operator $L f=\left\langle|d \varphi|^{-2} d \varphi\right.$, $d f\rangle$, by noting that $\frac{1}{i \tau} L\left(e^{i \tau \varphi}\right)=e^{i \tau \varphi}$ :

$$
\begin{aligned}
\int_{V_{j} \cap M_{0}} e^{i \tau \varphi} p^{(l)} \bar{q}^{\left(l^{\prime}\right)} \phi_{1} d V_{g}=\int_{V_{j} \cap \partial M_{0}} \frac{\partial_{\nu} \varphi}{i \tau|d \varphi|^{2}} e^{i \tau \varphi} p^{(l)} \bar{q}^{\left(l^{\prime}\right)} \phi_{1} d S & \\
& +\frac{1}{i \tau} \int_{V_{j} \cap M_{0}} e^{i \tau \varphi} L^{t}\left(p^{(l)} \bar{q}^{\left(l^{\prime}\right)} \phi_{1}\right) d V_{g}
\end{aligned}
$$

where $L^{t}$ is the transpose of the operator $L$. Now we have the job to estimate the two integrals on the right hand side; the proof of this is identical to the proof in [23]. By using the fact that $\int \tau^{\frac{n-2}{2}} e^{-c \tau|y|^{2}}|y|^{2} d y=O\left(\tau^{-1}\right)$ and that in the local chart determined by $l,\left|d \operatorname{Im} \Theta^{(l)}\right| \lesssim|y|$, we have:

$$
\left\|\left|d \operatorname{Im} \Theta^{(l)}\right| v^{(l)}\right\|_{L^{2}}\left\|\bar{\omega}^{\left(l^{\prime}\right)}\right\|\left\|\phi_{1}\right\|_{L^{\infty}} \lesssim \tau^{-1 / 2}
$$

But this is exactly the form of summand that contributes the most to the second integral; it is the one that is obtained upon acting of $L^{t}$ on $e^{-s \operatorname{Im} \Theta^{(l)}}$, because after differentiation we get an extra $\tau$ term which happily cancels with $\frac{1}{i \tau}$; everything else is bounded.

The boundary integral is bounded by previous local bounds; hence the $\frac{1}{i \tau}$ factor takes care of it. Therefore, finally, by using the previous case on each of the factors $v_{s}^{(l)} \bar{\omega}_{s}^{(l)}$, we have that:

$$
\lim _{\tau \rightarrow \infty} \int_{\left\{x_{1}^{\prime}\right\} \times M_{0}} v_{s}^{(l)} \bar{\omega}^{(l)} \phi d V_{g}=\int_{I^{(l)}} e^{-2 \lambda t} e^{\Phi_{1}+\bar{\Phi}_{2}} \phi d t
$$

So by adding these for time intervals $I^{(l)}$ for $\gamma\left(t_{l}\right)=p_{j}$, we get the desired result.

We are left with the final piece of the proof, which is concerned about the concentration properties of the solutions when coupled with a 1-form. As before, by using a partition of unity, we may assume $\phi$ has compact support in some of the $W_{k}$ or $V_{i}$ (the part of $\phi$ which is zero near the geodesic, can be made to have disjoint support with $v_{s}$ ). 
Let us first consider the case $\operatorname{supp}(\phi) \subset W_{k}$. Here we have $\left.v_{s}\right|_{W_{k}}=v^{(l)}=e^{i s \Theta^{(l)}} a^{(l)}$ and $\left.\omega_{s}\right|_{W_{k}}=\omega^{(l)}=e^{i s \Theta^{(l)}} b^{(l)}$ for some $l$. We want to compute the following limit, where we use the $x=(t, y)$ coordinates (we drop some of the indices):

$$
\begin{aligned}
\frac{1}{\tau} \int_{\left\{x_{1}^{\prime}\right\} \times M_{0}} g^{i j} \alpha_{i} \frac{\partial v_{s}}{\partial x^{j}} \bar{\omega}_{s} \phi d V_{g}= & \frac{i s}{\tau} \int_{\left\{x_{1}^{\prime}\right\} \times M_{0}} g^{i j} \alpha_{i} \frac{\partial \Theta}{\partial x^{j}} v_{s} \bar{\omega}_{s} \phi d V_{g} \\
& +\frac{1}{\tau} \int_{\left\{x_{1}^{\prime}\right\} \times M_{0}} g^{i j} \alpha_{i} e^{i s \Theta} \frac{\partial a}{\partial x^{j}} \bar{\omega}_{s} \phi d V_{g} \rightarrow \int_{0}^{L} i \alpha_{t} e^{\Phi_{1}+\bar{\Phi}_{2}} e^{-2 \lambda t} \phi d t
\end{aligned}
$$

as $\tau \rightarrow \infty$, where $\alpha_{t}=\alpha(\dot{\gamma}(t))$; this is because the first integral can be computed by our previous considerations and using the fact that $\Theta=t+1 / 2 \operatorname{Im} H(t) y \cdot y+O\left(|y|^{3}\right)$ to compute the derivatives along the geodesic. Furthermore, the second term goes to zero by this simple estimate:

$$
\left\|\omega_{s}\right\|_{L^{2}} \int_{0}^{L} \int_{\mathbb{R}^{n-2}}|\alpha|^{2}\left|e^{i s \Theta}\right|^{2}|d a|^{2} d y d t \lesssim \int_{0}^{L} \int_{\mathbb{R}^{n-2}} \tau^{\frac{n-2}{2}} e^{-\frac{1}{2} c \tau|y|^{2}} d y d t=O(1)
$$

which finishes the proof in this case.

For the more complicated case $\operatorname{supp}(\phi) \subset V_{k}$, we have that $v_{s}=\sum_{\gamma\left(t_{l}\right)=p_{k}} v_{s}^{(l)}$ and $\omega_{s}=$ $\sum_{\gamma\left(t_{l}\right)=p_{k}} \omega_{s}^{(l)}$. In the coordinates $x=(t, y)$ corresponding to $I^{(l)}$, for each $l$ and $l^{\prime}$ with $\gamma\left(t_{l}\right)=$ $\gamma\left(t_{l^{\prime}}\right)=p_{k}$ :

$$
\begin{aligned}
\int_{\left\{x_{1}^{\prime}\right\} \times M_{0}} g^{i j} \alpha_{i} \frac{\partial v^{(l)}}{\partial x^{j}} \bar{\omega}^{\left(l^{\prime}\right)} \phi d V_{g}=\frac{i s}{\tau} \int_{\left\{x_{1}^{\prime}\right\} \times M_{0}} g^{i j} \alpha_{i} \frac{\partial \Theta}{\partial x^{j}} v^{(l)} \bar{\omega}^{\left(l^{\prime}\right)} \phi d V_{g} & \\
& +\frac{1}{\tau} \int_{\left\{x_{1}^{\prime}\right\} \times M_{0}} g^{i j} \alpha_{i} e^{i s \Theta} \frac{\partial a}{\partial x^{j}} \bar{\omega}^{\left(l^{\prime}\right)} \phi d V_{g}
\end{aligned}
$$

where we write $v^{(l)}=e^{i s \Theta} a$. Now by the previous steps, we easily see that, if $l \neq l^{\prime}$, the first term is zero in the limit and the second term goes to zero by the bound (4.11) above. However, if we have $l=l^{\prime}$, by the previous step we again have the right limit, which is $\int_{I^{(l)}} i \alpha_{t} e^{\Phi_{1}+\bar{\Phi}_{2}} e^{-2 \lambda t} d t$. Combining the results, we obtain:

$$
\lim _{\tau \rightarrow \infty} \int_{\left\{x_{1}^{\prime}\right\} \times M_{0}}\left\langle d v_{s}, \alpha\right\rangle \bar{\omega}_{s} \phi d V_{g}=\sum_{\gamma\left(t_{l}\right)=p_{k}} \int_{I^{(l)}} i \alpha_{t} e^{\Phi_{1}+\bar{\Phi}_{2}} e^{-2 \lambda t} \phi d t=\int_{0}^{L} i \alpha_{t} e^{\Phi_{1}+\bar{\Phi}_{2}} e^{-2 \lambda t} \phi d t
$$

which finally finishes the proof. Similarly to this last part of the proof we can determine the limit where the integrand is $\left\langle\alpha, d \bar{\omega}_{s}\right\rangle v_{s} \phi$ - we get the same limit with just a minus sign in front.

REMARK 4.5. The equation 4.3 defining $\Phi_{1}$ is invariant under summing with an antiholomorphic function. Therefore, in the previous theorem, we could have inserted an extra antiholomorphic part $h$ in the integrand of the limit. Moreover, we can see from the proof (see (4.7) and the lines nearby) that we could have changed the estimate $\left\|e^{s x_{1}}(\Delta+X+q) e^{-s x_{1}} v_{s}\right\|_{L^{2}(M)}=$ $o(\tau)$ as $|\tau| \rightarrow \infty$ with the stronger, $O\left(|\tau|^{-K}\right)$ estimate, for any $K>0$ - this will get used in the partial boundary data setting.

REMARK 4.6. Note that we also have $\left\|d v_{s}\right\|_{L^{2}\left(M ; T^{*} M\right)}=O(|\tau|)$ (or equivalently $\left\|v_{s}\right\|_{H_{s c l}^{1}(M)}=$ $O(1)$ for $h=\frac{1}{\tau}$ ). This simply follows from the local estimate (4.6) and the fact that $d v_{s}=$ $i s(d \Theta) e^{i s \Theta} a+e^{i s \Theta} d a$ (locally), so in the end we just get an extra factor of $\tau$ in the $L^{2}(M)$ norm.

REMARK 4.7. It is also of interest to mention that the above construction works for metrics on $\mathbb{R} \times M_{0}$ that are conformal to the product metric (this is also considered in $[\mathbf{2 3}]$ ). However, for simplicity we have omitted this conformal factor from the statement of this theorem, but more importantly we can prove Theorem 4.10 without this fact. It is not essential at this point (it will 
be important later, when when we use the integral identity) that $X$ and $Y$ are skew-Hermitian, but the equation (4.1) is simpler with this assumption.

We are also interested in a vector valued version of the previous theorem. The statement of this theorem is completely analogous for vectors (matrices), as well as the proof; however, we give a sketch of the proof at some points of difference $\left(E^{\prime}=\mathbb{R} \times M_{0} \times \mathbb{C}^{m \times m}\right.$ is the vector bundle of matrices with the fibrewise Hermitian inner product $\left.\langle A, B\rangle=\operatorname{tr}\left(A B^{*}\right)\right)$.

Theorem 4.8 (Construction of the vector valued Gaussian Beams). Let $\gamma:[0, L] \rightarrow M_{0}$ be a non-tangential geodesic and let $\lambda$ be a real parameter, with $M_{0}$ any compact manifold with boundary. Let $X$ and $Y$ be two skew-Hermitian matrices of vector fields on $M$ and $q$ a matrix potential; we extend $X, Y$ and $q$ to have compact support in $\mathbb{R} \times M_{0}$. Let $N_{0}$ be a large positive integer and denote $J_{0}=\left[-N_{0}, N_{0}\right]$. Then there exists a family of generalised quasimodes satisfying the above conditions, i.e. if $s=\tau+i \lambda$, then there exists $v_{s}, \omega_{s} \in C^{\infty}\left(J_{0} \times M_{0}, E^{\prime}\right)$ such that:

$\left\|\left(\left(\Delta_{g}+X+q\right)+s\left(2 \frac{\partial}{\partial x_{1}}-X_{1}\right)-s^{2}\right) v_{s}\right\|_{L^{2}\left(J_{0} \times M_{0} ; E^{\prime}\right)}=o(|\tau|) \quad$ and $\quad\left\|v_{s}\right\|_{L^{2}\left(J_{0} \times M_{0} ; E^{\prime}\right)}=O(1)$
$\left\|\left(\left(\Delta_{g}+Y+q\right)-s\left(2 \frac{\partial}{\partial x_{1}}-Y_{1}\right)-s^{2}\right) \omega_{s}\right\|_{L^{2}\left(J_{0} \times M_{0} ; E^{\prime}\right)}=o(|\tau|) \quad$ and $\quad\left\|\omega_{s}\right\|_{L^{2}\left(J_{0} \times M_{0} ; E^{\prime}\right)}=O(1)$ as $\tau \rightarrow \infty$ and for each $\phi \in C\left(M_{0}\right)$ and $x_{1}^{\prime} \in \mathbb{R}$ we have:

$$
\lim _{\tau \rightarrow \infty} \int_{\left\{x_{1}^{\prime}\right\} \times M_{0}} \operatorname{tr}\left(v_{s} \omega_{s}^{*}\right) \phi d V_{g}=\int_{0}^{L} e^{-2 \lambda t} \operatorname{tr}\left(C_{X} C_{Y}^{*}\right) \phi(\gamma(t)) d t
$$

where $C_{X}$ and $C_{Y}$ are smooth $m \times m$ matrices on $\mathbb{R} \times[0, L]$ which satisfy the following equations:

$$
\left(\frac{\partial}{\partial x_{1}}-i \frac{\partial}{\partial r}\right)\left(C_{X}\right)=\frac{1}{2}\left(X_{1}-i X_{r}\right) C_{X} \quad \text { and } \quad\left(\frac{\partial}{\partial x_{1}}-i \frac{\partial}{\partial r}\right)\left(C_{Y}^{*}\right)=\frac{1}{2} C_{Y}^{*}\left(-Y_{1}+i Y_{r}\right)
$$

Moreover, the following limits holds for $v_{s}$ and $\omega_{s}$ and any one form $\alpha$ on $\mathbb{R} \times M_{0}$ :

$$
\begin{aligned}
& \lim _{\tau \rightarrow \infty} \frac{1}{\tau} \int_{\left\{x_{1}^{\prime}\right\} \times M_{0}} \operatorname{tr}\left(\left\langle\alpha, d v_{s}\right\rangle \omega_{s}^{*}\right) \phi d V_{g}=\int_{0}^{L} i \alpha(\dot{\gamma}(t)) \operatorname{tr}\left(C_{X} C_{Y}^{*}\right) e^{-2 \lambda t} \phi(\gamma(t)) d t \\
& \lim _{\tau \rightarrow \infty} \frac{1}{\tau} \int_{\left\{x_{1}^{\prime}\right\} \times M_{0}} \operatorname{tr}\left(\left\langle\alpha, d \omega_{s}^{*}\right\rangle v_{s}\right) \phi d V_{g}=-\int_{0}^{L} i \alpha(\dot{\gamma}(t)) \operatorname{tr}\left(C_{X} C_{Y}^{*}\right) e^{-2 \lambda t} \phi(\gamma(t)) d t
\end{aligned}
$$

Proof. Same as the proof of Theorem 4.4, with a few remarks. Firstly, every appearance of $v_{s} \bar{\omega}_{s}$ is replaced by the inner product $\operatorname{tr}\left(v_{s} \omega_{s}^{*}\right)$ and we are looking for $v_{s}=e^{i s \Theta} a$, where this time $a$ is a matrix; so the action of $X$ and $Y$ is matrix multiplication from the left. However, formally, the computations stay the same until the appearance of $\Phi_{1,2}$; the $C_{X, Y}$ take their role, this time as matrices. Namely, when we arrive to the equation for $a_{00}$, which is 4.2 :

$$
\left(\frac{\partial}{\partial x_{1}}-i \frac{\partial}{\partial t}\right) a_{00}=\frac{1}{2}\left(X_{1}-i X_{t}+i \operatorname{tr} H(t)\right) a_{00}
$$

we ask for matrices $C_{X}$ and $C_{1}$ such that $a_{00}=C_{X} C_{1}$, where:

$$
\left(\frac{\partial}{\partial x_{1}}-i \frac{\partial}{\partial t}\right) C_{X}=\frac{1}{2}\left(X_{1}-i X_{t}\right) C_{X} \quad \text { and } \quad \frac{\partial C_{1}}{\partial t}=-\frac{1}{2} \operatorname{tr}(H(t)) C_{1}(t)
$$

so that $C_{1,2}$ play the role of $f_{1,2}$. One checks that such $a_{00}$ satisfies the conditions and for $C_{1}(t)$ we just take the diagonal matrix obtained by integration. This is later used to get the cancellation of $\sqrt{\operatorname{det} \operatorname{Im} H(t)}$ with $C_{1} C_{2}^{*}$, which jumps out of the trace as before in the integral 4.10 .

Later, when proving the mixed products vanish, the $p$ 's and $q$ 's introduced translate to matrices naturally and the estimates which follow stay the same. Finally, let us note that $C_{X}$ 
is invariant under multiplication on the right by an anti-holomorphic (conjugate holomorphic) matrix in the sense we could replace $C_{X}$ by $C_{X} H$ for such a matrix $H$.

REMARK 4.9 (Everything works for admissible vector bundles). We can now easily extend the construction from the case of trivial vector bundles to the case of possibly topologically non-trivial admissible ones (see Definition $\mathrm{D}$, equipped by a unitary connection. We restrict our attention just to operators $d_{A}^{*} d_{A}+Q$ induced by connections and potentials; to this end, assume the vector bundle $E=\pi^{*} E_{0}$ over $\mathbb{R} \times M_{0}$ is equipped with two unitary connections $A_{1}$ and $A_{2}$, where $E_{0}$ is a vector bundle over $M_{0}$.

Basically, what we need to do is to imitate the above vector proof with small alterations: to start with, let us recall the Fermi coordinates given by a map $F: J_{0} \times U \rightarrow \mathbb{R} \times M_{0}$, where $U=[-\epsilon, \epsilon+L] \times B_{\delta}$ and $B_{\delta}$ is a small ball in dimension $(n-2)-F$ is a local diffeomorphism, giving us the tubular neighbourhood of the geodesic (see Figure 1). Therefore, we can pull-back the bundle $E$ to the trivial bundle $F^{*} E=U \times \mathbb{C}^{m}$ with the standard metric; we pull back the connections and the metric, as well. Furthermore, in this case we cannot work on End $E$ as we previously did in Section 6.2. This means we have to restrict to vector solutions and in particular our solutions to the transport equation that go into the Gaussian beams will be vectors. Then we may run the proof again; the only thing we need to replace are the resulting concentration properties:

$$
\lim _{\tau \rightarrow \infty} \int_{\left\{x_{1}^{\prime}\right\} \times M_{0}}\left\langle v_{s}, \omega_{s}\right\rangle_{E} \phi d V_{g}=\int_{0}^{L} e^{-2 \lambda t}\left\langle C_{1} a_{1}, C_{2} a_{2}\right\rangle_{\mathbb{C}^{m}} \phi(\gamma(t)) d t
$$

where $C_{1}$ and $C_{2}$ are constructed on $J_{0} \times U$ for connections $A_{1}$ and $A_{2}$ as fundamental solutions to the $\bar{\partial}$-equation (4.12), respectively; the $a_{1}$ is anti-holomorphic so that $C_{1} a_{1}$ solves the vector $\partial$-equation and $a_{2}$ is analogously holomorphic, so that $C_{2} a_{2}$ solves the $\bar{\partial}$-equation. Then we may in particular set $a_{i}$ to be constant and vary these constants to deduce various properties.

For the other identity we have to be slightly more careful; namely $d v_{s}$ is not well defined as for the trivial bundle. However, we may define it as $d v_{s}$ in our construction in $U$ and then push it forward by the same method of partition of unity and the map $F$ to the neighborhood of the geodesic (as in (4.8) ) and hence to the whole manifold as a 1-form with values in $E$ (and with support in a neighbourhood of the geodesic). Then the identities become:

$$
\begin{aligned}
& \lim _{\tau \rightarrow \infty} \frac{1}{\tau} \int_{\left\{x_{1}^{\prime}\right\} \times M_{0}}\left\langle\left\langle\alpha, d v_{s}\right\rangle_{T^{*} M}, \omega_{s}\right\rangle_{E} \phi d V_{g}=\int_{0}^{L} i \alpha(\dot{\gamma}(t))\left\langle C_{1} a_{1}, C_{2} a_{2}\right\rangle_{\mathbb{C}^{m}} e^{-2 \lambda t} \phi(\gamma(t)) d t \\
& \lim _{\tau \rightarrow \infty} \frac{1}{\tau} \int_{\left\{x_{1}^{\prime}\right\} \times M_{0}}\left\langle\left\langle\alpha, d \omega_{s}\right\rangle_{T^{*} M}, v_{s}\right\rangle_{E} \phi d V_{g}=\int_{0}^{L} i \alpha(\dot{\gamma}(t))\left\langle C_{1} a_{1}, C_{2} a_{2}\right\rangle_{\mathbb{C}^{m}} e^{-2 \lambda t} \phi(\gamma(t)) d t
\end{aligned}
$$

\subsection{Application of Gaussian Beams}

We now give a concrete application of the construction of generalised quasimodes - the construction of the CGO solutions. By using the Carleman estimates from Chapter 5, we can just put the ingredients together in a simple way. For this section, assume we are working in the setting of the CTA manifolds, that is $\tilde{g}=e \oplus g_{0}$ with $g=c \tilde{g}$ for a positive function $c$, where as usual we have $(M, g) \Subset\left(\mathbb{R} \times M_{0}, g\right)$ of the same dimension $n$.

Proposition 4.10 (CGO construction). Let $E$ be an admissible Hermitian vector bundle, $A$ a unitary connection and $Q$ be a smooth section of the endomorphism bundle End $E$. Let $s=\tau+i \lambda$, where $\tau$ and $\lambda$ are real numbers. Then there exists $\tau_{0}$, such that for $|\tau| \geq \tau_{0}$ large enough, there exists a smooth solution $u=e^{-s x_{1}} c^{-\frac{n-2}{4}}\left(v_{s}+r_{s}\right)$ to the equation $\mathcal{L}_{g, A, Q} u=0$, 
with the following conditions fulfilled:

$$
\left\|r_{s}\right\|_{L^{2}(M ; E)}=o(1), \quad\left\|r_{s}\right\|_{H^{1}(M ; E)}=o(|\tau|) \quad \text { and } \quad\left\|v_{s}\right\|_{L^{2}(M ; E)}=O(1)
$$

as $|\tau| \rightarrow \infty$ and the concentration properties for $v_{s}$ as in Theorem 4.4.

Proof. Let us firstly notice the identity:

$$
c^{\frac{n+2}{4}} \mathcal{L}_{g, A, Q}(u)=\mathcal{L}_{\tilde{g}, A, c\left(Q+Q_{c}\right)}\left(e^{-s x_{1}}\left(v_{s}+r_{s}\right)\right)
$$

where $Q_{c}=c^{\frac{n-2}{4}} \Delta_{g}\left(c^{-\frac{n-2}{4}}\right)$. Therefore, if we let $v_{s}$ be the function constructed in the proof of Theorem 4.4, with all its concentration properties, we will have

$$
\left\|e^{s x_{1}} \mathcal{L}_{\tilde{g}, A, c\left(Q+Q_{c}\right)} e^{-s x_{1}} v_{s}\right\|_{L^{2}(M ; E)}=o(|\tau|)
$$

Hence, to have the required form of the solution, $r_{s}$ must satisfy

$$
e^{\tau x_{1}} \mathcal{L}_{g, A, Q} e^{-\tau x_{1}}\left(c^{-\frac{n-2}{4}} e^{-i \lambda x_{1}} r_{s}\right)=-c^{-\frac{n+2}{4}} e^{-i \lambda x_{1}} e^{s x_{1}} \mathcal{L}_{\tilde{g}, A, c\left(Q+Q_{c}\right)} e^{-s x_{1}} v_{s}
$$

But fortunately, now the right hand side is $o(|\tau|)$ by construction and $c$ is bounded, hence we may apply the existence theorem - Theorem 5.5 .

REMARK 4.11. Note that in Theorem 4.10 we can do better with the estimate on the $H^{1}(M ; E)$ norm of $r_{s}$, by invoking Remark 4.5 and the improved estimate on the asymptotics of $\left\|e^{s x_{1}} \mathcal{L}_{A, Q} e^{-s x_{1}} v_{s}\right\|_{L^{2}(M ; E)}=O\left(|\tau|^{-K}\right)$ for any $K \geq 0$. Moreover, this implies that with the improved estimate on $v_{s}$ we have the $L^{2}$ norm of the right hand side of 4.13 equal to $O\left(|\tau|^{-K}\right)$ and consequently, by Theorem 5.5 , we have:

$$
\left\|r_{s}\right\|_{L^{2}(M ; E)}=O\left(|\tau|^{-(K+1)}\right) \quad \text { and } \quad\left\|r_{s}\right\|_{H^{1}(M ; E)}=O\left(|\tau|^{-K}\right)
$$

or equivalently, $H_{s c l}^{1}(M ; E)=O\left(|\tau|^{-(K+1)}\right)$.

REMARK 4.12. Having been through the lengthy proof of existence of Gaussian Beams in case of the connection Laplacian, we now give an alternative idea on how to generalise the notion of quasimodes. Namely, it is natural to attempt to construct the analogous quantity to the approximate eigenfunction that satisfies $\left\|\left(\Delta-s^{2}\right) v_{s}\right\|_{L^{2}\left(M_{0}\right)}=o(|\tau|)$ by asking that $\left\|\left(-d_{A}^{*} d_{A}-s^{2}\right) v_{s}\right\|_{L^{2}\left(M_{0}\right)}=o(|\tau|)$. However, by generalising in this way, we lose the purpose of it: we cannot build the CGO solutions using such construction. Thus, even though the construction of such solutions should be possible and completely analogous to our main construction, we cannot find any application for it. 



\section{CHAPTER 5}

\section{Carleman estimates}

The purpose of this chapter is to prove suitable Carleman estimates for vector valued functions. The scalar case was covered in $[\mathbf{2 1}]$ and we generalise that approach, as expected since the principal part of $d_{A}^{*} d_{A}$ is diagonal.

Firstly, let us briefly explain what the limiting Carleman weights (LCW) are. These are certain functions on open Riemannian manifolds that guarantee the positivity of the conjugated Laplacian operator $P_{0, \varphi}=e^{\frac{\varphi}{h}} \Delta e^{-\frac{\varphi}{h}}$ and hence existence of solutions to equations as below. They were introduced in [40] for the Euclidean case and generalised to manifolds in [21]. They have a nice geometric characterisation: in $[\mathbf{2 1}$ it is proved that the existence of LCW is equivalent to existence of a unit parallel vector field on the manifold (a vector field $V$ is parallel if $\nabla V=0$, where $\nabla$ is the Levi-Civita connection). This vector field yields a Euclidean direction on the manifold - hence, for simplicity, we will often assume our manifold to be embedded in $\mathbb{R} \times M_{0}$, which admits the Carleman weight $\varphi(x)=x_{1}$.

Moreover, one way to motivate the definition of LCWs is that its reverse engineered so that the estimates below in Theorem 5.2 hold for both $\pm \varphi$ (the proof of the converse to this statement, i.e. that the inequality holds for both $\pm \varphi$ implies that $\varphi$ is an LCW is outlined in $[\mathbf{2 0}]$ ), so that the two solutions constructed in Proposition 4.10 with the corresponding phases equal to $\pm x_{1}$, cancel out in the integral identity from Theorem 2.6. We state the definition of an LCW here.

DeFinition 5.1. Let $(U, g)$ be an open Riemannian manifold. We say $(U, g)$ admits an $L C W$ if there exists a smooth $\varphi: U \rightarrow \mathbb{R}$, such that $d \varphi \neq 0$ and if we let $p_{\varphi}$ to be the semiclassical principal symbol of $P_{0, \varphi}$, then:

$$
\left\{\operatorname{Re} p_{\varphi}, \operatorname{Im} p_{\varphi}\right\}(x, \xi)=0 \quad \text { when } p_{\varphi}(x, \xi)=0
$$

where $\{\cdot, \cdot\}$ is the Poisson bracket on $T^{*} U$.

In the text below, we denote by $H_{s c l}^{1}(M ; E)$ the semiclassical Sobolev space associated to the sections of the Hermitian vector bundle $E$ of rank $m$ over $M$, equipped with a connection $\nabla$, with the norm:

$$
\|u\|_{H_{s c l}^{1}(M ; E)}=\left(\|u\|_{L^{2}(M ; E)}^{2}+h^{2}\|\nabla u\|_{L^{2}\left(M ; T^{*} M \otimes E\right)}^{2}\right)^{\frac{1}{2}}
$$

and by $L^{2}(M ; E)$ the inner product space associated with the Hermitian structure and the Riemannian density (see also Appendix A for alternative definitions). We start by proving a warm-up a priori Carleman estimate which relates the $H_{s c l}^{1}$ and $L^{2}$ norms of a solution to $P_{0, \varphi} u=f$, by essentially using only elementary methods; later we will see, in order to obtain a $H^{1}$ solution, we have to shift the indices and prove the inequality for every $H_{s c l}^{s}$, where $s \in \mathbb{R}$.

Let us introduce the setting in which the theorems will be proved. We will work on $M$, a compact manifold with boundary which is compactly contained in $U$, an open manifold admitting a Carleman weight $\varphi$; moreover, $U$ is again contained in a closed manifold $N$, which is useful since then we do not have to worry about boundary conditions on $N$ (we may let $N$ to be the double of $M)$. We assume there is an extension of the Hermitian vector bundle $E$ over $M$ to a 
bundle over $N$, denoted by the same letter; we equip $E$ with a connection $A$ and a section $Q$ of the endomorphism bundle.

TheOrem 5.2. Let $X$ be a smooth matrix of vector fields on $M$ and $q$ a smooth matrix function on $M$ (matrices are $m$ by $m$ ). Then there exists a constant $C$, such that the following inequality holds for all $u \in C_{c}^{\infty}\left(M^{\text {int }} ; \mathbb{C}^{m}\right)$ and all sufficiently small $h>0$ :

$$
\left\|e^{\varphi / h} u\right\|_{H_{s c l}^{1}\left(M ; \mathbb{C}^{m}\right)} \leq C h\left\|e^{\varphi / h}(\Delta+X+q) u\right\|_{L^{2}\left(M ; \mathbb{C}^{m}\right)}
$$

Moreover, the following inequality holds for all $u \in C_{c}^{\infty}\left(M^{\text {int }} ; E\right)$ :

$$
\left\|e^{\varphi / h} u\right\|_{H_{s c l}^{1}(M ; E)} \leq C^{\prime} h\left\|e^{\varphi / h}\left(d_{A}^{*} d_{A}+Q\right) u\right\|_{L^{2}(M ; E)}
$$

Proof. We prove the first inequality; the second one follows by a partition of unity argument in $N$ and applying the first inequality, since locally $d_{A}^{*} d_{A}+Q$ is of the form $\Delta+X+q$ (see $(2.8)$ ).

Firstly, notice we have invariance under conformal scaling, i.e. observe that we have the identity:

$$
c^{\frac{n+2}{4}}\left(\Delta_{g}+X+q\right) u=\left(\Delta_{c^{-1} g}+c X+q_{c}\right)\left(c^{\frac{n-2}{4}} u\right)
$$

where $q_{c}=c q-\frac{n-2}{4} X c+c^{\frac{n+2}{4}} \Delta\left(c^{\frac{n-2}{4}}\right)$, by using the conformal properties of the Laplacian. From this we can easily deduce the invariance of the inequality under conformal scaling, because we have:

$$
\begin{gathered}
h\left\|e^{\varphi / h}(\Delta+X+q) e^{-\varphi / h} u\right\|_{L^{2}}=h\left\|e^{\varphi / h} c^{-\frac{n+2}{4}}\left(\Delta_{c^{-1} g}+c X+q_{c}\right) e^{-\varphi / h}\left(c^{\frac{n-2}{4}} u\right)\right\|_{L^{2}} \\
\gtrsim\left\|c^{\frac{n-2}{4}} u\right\|_{H_{s c l, c^{-1} g}^{1}}=\left\|c^{\frac{n-2}{4}} u\right\|_{L^{2}}+h\left(\int_{M}\left\langle d\left(c^{\frac{n-2}{4}} u\right), d\left(c^{\frac{n-2}{4}} u\right)\right\rangle\right)^{1 / 2} \\
\geq \min c^{\frac{n-2}{4}}\|u\|_{L^{2}}+h\left\|d\left(c^{\frac{n-2}{4}}\right) u+c^{\frac{n-2}{4}} d u\right\|_{L^{2}, c^{-1} g} \\
\geq \min c^{\frac{n-2}{4}}\|u\|_{L^{2}}+h \min c^{\frac{n+2}{4}}\|d u\|_{L^{2}, g}-h \max \left|d\left(c^{\frac{n-2}{4}}\right)\right|_{g}\|u\|_{L^{2}}
\end{gathered}
$$

where by $H_{s c l, g^{\prime}}^{1}$ we denote the norm associated to some metric $g^{\prime}$. Now, for $h$ small enough, we must have $\min c^{\frac{n-2}{4}}>h \max c\left|d\left(c^{\frac{n-2}{4}}\right)\right|_{g}$, which shows the reduction step. We can now freely assume that $\nabla \varphi$ has unit norm, as conformal scalings preserve the property of being a LCW. In other words we may assume that the function $\varphi$ is a distance function, i.e. we have $|\nabla \varphi|=1$ and $D^{2} \varphi=0$, where $D$ is the Levi-Civita covariant derivative (see Lemma 2.5 in $[\mathbf{2 1}$ ) . 1

Furthermore, if we assume the inequality holds for $q=0$ :

$$
\begin{aligned}
C h\left\|e^{\varphi / h}(\Delta+X+q) e^{-\varphi / h} u\right\|_{L^{2}} & \geq C h\left\|e^{\varphi / h}(\Delta+X) e^{-\varphi / h} u\right\|_{L^{2}}-C h\|q u\|_{L^{2}} \\
& \geq\|u\|_{H_{s c l}^{1}(M)}-C h \max \|q\|\|u\|_{L^{2}}
\end{aligned}
$$

As soon as $h$ is small enough such that $C h \max \|q\|<1$, we may absorb the remaining factor, so we are reduced to proving the $q=0$ case. Here we have denoted by $\|q\|$ the operator norm of $q$ and the maximum is over all of $M$.

In this step we show the inequality under the additional assumption that $X=0$. Recall the following identity, with the specific expansion we will make use of later:

$$
P_{0, \varphi}=e^{\varphi / h} h^{2} \Delta_{g} e^{-\varphi / h}=\underbrace{h^{2} \Delta-|\nabla \varphi|^{2}}_{A}+\underbrace{2\langle\nabla \varphi, h \nabla\rangle-h \Delta \varphi}_{i B}
$$

\footnotetext{
$\overline{{ }^{1} \mathrm{In}} \overline{\mathbf{2 1}}$ it is also proved that a distance function is also an LCW if and only if $\varphi\left(\exp _{x} v\right)=\varphi(x)+\langle\nabla \varphi(x), v\rangle$; in particular, this means that we have a lot of LCWs in the Euclidean spaces, by letting $\varphi(x)=\rho \cdot x$ for a vector $\rho$.
} 
Hence, we can build the following estimates (we leave out the $L^{2}$ subscript for convenience):

$$
\left(P_{0, \varphi} v, v\right)=h^{2}(\Delta v, v)-\left(|\nabla \varphi|^{2} v, v\right)+2 h(\langle\nabla \varphi, \nabla v\rangle, v)-h(\Delta \varphi v, v)
$$

By using the fact that $\int\langle d f, d g\rangle=(\Delta f, g)$ for $f$ and $g$ compactly supported, we get:

$$
\|h \nabla v\|^{2}=\left(P_{0, \varphi} v, v\right)+\left(|\nabla \varphi|^{2} v, v\right)-2 h(\langle\nabla \varphi, \nabla v\rangle, v)+h(\Delta \varphi v, v)
$$

Therefore, we finally have, using Cauchy-Schwartz and AM-GM:

$$
\begin{aligned}
\|h \nabla v\|^{2} & \leq\left\|P_{0, \varphi} v\right\|\|v\|+\|v\|^{2}+2\|v\|\|h \nabla v\|+h|\sup \Delta \varphi|\|v\|^{2} \\
& \leq \frac{1}{2}\left\|P_{0, \varphi}\right\|^{2}+\frac{1}{2}\|v\|^{2}+\frac{1}{\epsilon}\|v\|^{2}+\epsilon\|h \nabla v\|^{2}
\end{aligned}
$$

So for some $C_{1}$ and sufficiently small $\epsilon$ :

$$
\|h \nabla v\|^{2} \leq\left\|P_{0, \varphi} v\right\|^{2}+C_{1}\|v\|^{2}
$$

Therefore, it suffices to prove $\|v\| \leq C_{2} h^{-1}\left\|P_{0, \varphi} v\right\|$ for some $C_{2}$.

Now, we claim that in the above expansion of $P_{0, \varphi}$, the parts $A$ and $B$ are formally selfadjoint. The proof is not too hard, but we give one for completeness. The bilinear map $\langle\cdot, \cdot\rangle$ we use is complex bilinear; also, formal self-adjointness means $(P \varphi, \psi)=(\varphi, P \psi)$ for all smooth compactly supported functions $\varphi$ and $\psi$. We have, for $m=1$ :

$$
\left(\left(h^{2} \Delta-|\nabla \varphi|^{2}\right) u, v\right)=\left(u,\left(h^{2} \Delta-|\nabla \varphi|^{2}\right) v\right)
$$

for all $u, v \in C_{c}^{\infty}\left(M^{i n t}\right)$ because $\varphi$ is real and $\Delta$ is self-adjoint. Moreover, we have:

$$
\begin{aligned}
(2\langle\nabla \varphi, h \nabla u\rangle, v)=2 h(\langle d \varphi, d u\rangle, v) & =2 h \int\langle d \varphi, \bar{v} d u\rangle \\
= & 2 h \int\langle d \varphi, d(u \bar{v})-u d \bar{v}\rangle=2 h \int \Delta \varphi u \bar{v}-2 h(u,\langle\nabla \varphi, \nabla v\rangle)
\end{aligned}
$$

and $(h \Delta \varphi u, v)=h(u, \Delta \varphi v)$. Therefore, by combining the two results:

$$
\begin{aligned}
(2\langle\nabla \varphi, h \nabla u\rangle-h \Delta \varphi u, v)=2 h(\Delta \varphi u, v)- & h(\Delta \varphi u, v)-2 h(u,\langle\nabla \varphi, \nabla v\rangle) \\
& =-(u,(-h \Delta \varphi v+2 h\langle\nabla \varphi, \nabla v\rangle))=-(u, i B v)
\end{aligned}
$$

which finally implies that $A$ and $B$ are formally self-adjoint in the scalar case. For the $m>1$ case we just observe that the action of the Laplacian $\Delta$ extends diagonally to vector valued functions and the inner product $\langle u, v\rangle=\sum u_{i} \bar{v}_{i}$ splits nicely with respect to this action, so we can simply sum over components.

We will now make use of the following identity:

$$
\left\|P_{0, \varphi} v\right\|^{2}=\left(P_{0, \varphi} v, P_{0, \varphi} v\right)=((A+i B) v,(A+i B) v)=\|A v\|^{2}+\|B v\|^{2}+(i[A, B] v, v)
$$

The idea is to use the positivity of the principal symbol to deduce the positivity of the last term in the expression above. We first need to use a convexification argument (see $[\mathbf{2 1})$, where we slightly perturb $\varphi$ by a convex function. Namely, we consider a function $f: \mathbb{R} \rightarrow \mathbb{R}$ and the composition $\tilde{f}=f \circ \varphi$. Then we have:

- $P_{0, \tilde{f}}=\tilde{A}+i \tilde{B}$, according to the above decomposition.

- $\nabla(f \circ \varphi)=f^{\prime} \circ \varphi \nabla \varphi$.

- $D^{2}(f \circ \varphi)=D\left(f^{\prime} \circ \varphi d \varphi\right)=d\left(f^{\prime} \circ \varphi\right) \otimes d \varphi+f^{\prime} \circ \varphi D(d \varphi)=f^{\prime \prime} \circ \varphi d \varphi \otimes d \varphi+f^{\prime} \circ \varphi D^{2} \varphi$, where we used the fact that $\varphi$ is a distance function. 
Now we quote Lemma 2.3 from [21, which computes the Poisson bracket of the principal symbols of $A$ and $B$, which are respectively denoted as $a$ and $b$ :

$$
\{a, b\}(x, \xi)=4 D^{2} \varphi\left(\xi^{\#}, \xi^{\#}\right)+4 D^{2} \varphi(\nabla \varphi, \nabla \varphi)
$$

where we have the expressions $a=|\xi|^{2}-|d \varphi|^{2}=\left|\xi^{\#}\right|^{2}-|\nabla \varphi|^{2}$ and $b=2\left\langle\nabla \varphi, \xi^{\#}\right\rangle$. By $\alpha^{\#}$ we denote the unique element of $T_{p} M$ such that $\alpha(v)=\left\langle\alpha^{\#}, v\right\rangle$ for all $v$. With this notation, $a+i b=p_{\varphi}$ is the principal symbol of $P_{0, \varphi}$ in the standard semiclassical quantisation. Using the result of this lemma, we have for $m=1$ :

$$
\begin{aligned}
\{\tilde{a}, \tilde{b}\}(x, \xi) & =4\left(f^{\prime \prime} \circ \varphi\right)\left\langle\nabla \varphi, \xi^{\#}\right\rangle^{2}+4\left(f^{\prime \prime} \circ \varphi\right)\left(f^{\prime} \circ \varphi\right)^{2}|\nabla \varphi|^{4} \\
& =4\left(f^{\prime \prime} \circ \varphi\right)\left(f^{\prime} \circ \varphi\right)^{2}+\underbrace{\left(f^{\prime \prime} \circ \varphi\right)\left(f^{\prime} \circ \varphi\right)^{-2}}_{\beta} \tilde{b}^{2}
\end{aligned}
$$

where $\tilde{b}=2\langle d(f \circ \varphi), \xi\rangle=2\left(f^{\prime} \circ \varphi\right)\left\langle\nabla \varphi, \xi^{\#}\right\rangle$. So, we must have

$$
i[\tilde{A}, \tilde{B}]=4 h\left(f^{\prime \prime} \circ \varphi\right)\left(f^{\prime} \circ \varphi\right)^{2}+h \tilde{B} \beta \tilde{B}+h^{2} R
$$

where $R$ is first order semiclassical differential operator. Now we pick $f$ such that:

- $f(s)=s+\frac{h}{2 \epsilon} s^{2}, f^{\prime}(s)=1+\frac{h}{\epsilon} s$ and $f^{\prime \prime}=\frac{h}{\epsilon}$.

- Take $1 \geq \epsilon_{0} \geq \frac{h}{\epsilon}>0$ small enough such that $f^{\prime}>1 / 2$ on $\varphi(M)$ and denote $\varphi_{\epsilon}=f \circ \varphi$. One can check that the coefficients of $R$ are uniformly bounded with respect to $h$ and $\epsilon$, and $\beta=\frac{h / \epsilon}{\left(1+\frac{h}{\epsilon} s\right)^{2}}$ is uniformly bounded.

Namely, one has:

$$
\begin{aligned}
(i[\tilde{A}, \tilde{B}] v, v) & =\left(4 \frac{h^{2}}{\epsilon}\left(f^{\prime} \circ \varphi\right)^{2} v+h \tilde{B}\left(\frac{h / \epsilon}{\left(f^{\prime} \circ \varphi\right)^{2}} \tilde{B} v\right), v\right)+h^{2}(R v, v) \\
& \geq \frac{h^{2}}{\epsilon}\|v\|^{2}-C_{0} h\|\tilde{B} v\|^{2}-C_{0} h^{2}\|v\|_{H_{s c l}^{1}}\|v\|_{L^{2}}
\end{aligned}
$$

because $\|R v\| \leq C_{0}\|v\|_{H_{s c l}^{1}}$. The previous inequality hold for $m>1$, as $[\tilde{A}, \tilde{B}]$ acts diagonally, so $(i[\tilde{A}, \tilde{B}] v, v)_{L^{2}\left(\mathbb{C}^{m}\right)}=\sum\left(i[\tilde{A}, \tilde{B}] v_{j}, v_{j}\right)_{L^{2}}$.

Using the inequality $\|h \nabla v\|^{2} \leq\left\|P_{0, \varphi_{\epsilon}}\right\|^{2}+C_{1}\|v\|^{2}$, we conclude:

$$
(i[\tilde{A}, \tilde{B}] v \mid v) \geq \frac{h^{2}}{\epsilon}\left(1-C_{4} \epsilon\right)\|v\|^{2}-C_{3} h\|\tilde{B} v\|^{2}-C_{3}\left\|P_{0, \varphi_{\epsilon}}\right\|^{2}
$$

by employing $\|v\|_{H_{s c l}^{1}}=\|v\|_{L^{2}}+\|h \nabla v\|_{L^{2}} \leq C_{1}^{\prime} \cdot\|v\|_{L^{2}}+\left\|P_{0, \varphi_{\epsilon}}\right\|_{L^{2}}$ and AM-GM. Hence, we finally get the inequality:

$$
\left(1+C_{3}\right)\left\|P_{0, \varphi_{\epsilon}} v\right\|^{2} \geq\|\tilde{A} v\|^{2}+\left(1-C_{3} h\right)\|\tilde{B} v\|^{2}+\frac{h^{2}}{\epsilon}\left(1-C_{4} \epsilon\right)\|v\|^{2}
$$

Let us now turn to the case $X \neq 0$ - we want to incorporate it into the inequality (5.4) and to estimate it in a suitable way. Note that we have $h^{2} X_{\varphi_{\epsilon}}=h e^{\varphi_{\epsilon} / h} X e^{-\varphi_{\epsilon} / h}=h^{2} X-h f^{\prime} \circ \varphi X \varphi$. Thus we have:

- $\left\|h^{2} X v\right\|_{L^{2}}=\left\|h^{2}\langle X, \nabla v\rangle\right\|_{L^{2}} \leq h|X|_{L^{\infty}}\|h \nabla v\|_{L^{2}} \leq C_{2}^{\prime} \cdot h\|v\|_{H_{s c l}^{1}}$

- $\left\|h\left(f^{\prime} \circ \varphi\right) X(\varphi) v\right\|_{L^{2}} \leq C_{3}^{\prime}\|v\|_{L^{2}}$

By combining the two inequalities above, we conclude, by using (5.3):

$$
\left\|h^{2} X_{\varphi_{\epsilon}}\right\| \lesssim h\|v\|_{H_{s c l}^{1}} \lesssim h\left(\|v\|_{L^{2}}+\left\|P_{0, \varphi} v\right\|_{L^{2}}\right)
$$


which in turn implies the following chain of inequalities:

$$
\begin{aligned}
2\left(1+C_{3}\right)\left\|P_{0, \varphi_{\epsilon}} v+h^{2} X_{\varphi_{\epsilon}} v\right\|^{2} & \geq \frac{4}{3}\left(1+C_{3}\right)\left\|P_{0, \varphi_{\epsilon}} v\right\|^{2}-C_{5} h\left(\|v\|^{2}+\left\|P_{0, \varphi_{\epsilon}}\right\|^{2}\right) \\
& \geq\|v\|^{2}\left(\frac{h^{2}}{\epsilon}\left(1-C_{6} \epsilon\right)\right)
\end{aligned}
$$

where $C_{6}=C_{4}+C_{5}$. So for $\epsilon$ small enough, there exists $C_{7}$ such that:

$$
C_{7}\left\|P_{0, \varphi_{\epsilon}} v+h^{2} X_{\varphi_{\epsilon}} v\right\|^{2} \geq \frac{h^{2}}{\epsilon}\|v\|^{2}
$$

Therefore we have for $u=e^{-\varphi_{\epsilon} / h} v$ :

$$
C_{\epsilon} h^{2}\left\|e^{\frac{\varphi^{2}}{2 \epsilon}} e^{\frac{\varphi}{\epsilon}}(\Delta+X) u\right\|^{2} \geq\left\|e^{\frac{\varphi^{2}}{2 \epsilon}} e^{\frac{\varphi}{h}} u\right\|^{2}
$$

which together with $1 \leq e^{\frac{\varphi^{2}}{2 \epsilon}} \leq C_{\epsilon}^{\prime}$ implies the result.

REMARK 5.3. (Carleman estimates with a boundary term). We record a corollary of the above inequality for functions not necessarily supported in the interior of our manifold; this extends the inequality (2.13) from 22 to the higher rank case. Let $v \in C^{\infty}\left(M ; \mathbb{C}^{m}\right) \cap H_{0}^{1}\left(M ; \mathbb{C}^{m}\right)$ - then we claim that the following inequality holds:

$$
\|v\|_{H_{s c l}^{1}}^{2} \lesssim h^{2}\left\|e^{\frac{\varphi}{h}}(\Delta+X+q) e^{-\frac{\varphi}{h}} v\right\|^{2}+h\left(\partial_{\nu} \varphi \partial_{\nu} v, \partial_{\nu} v\right)_{\partial M}
$$

This is an exercise in partial integration and using the condition that $\left.v\right|_{\partial M}=0$ to get rid of the extra factors. Namely, what we get in the above notation is:

$$
\begin{aligned}
\|(A+i B) v\|^{2} & =\|A v\|^{2}+\|B v\|^{2}+i(B v, A v)-i(A v, B v) \\
& =\|A v\|^{2}+\|B v\|^{2}+i([A, B] v, v)-\left.2 h^{3}\left(\partial_{\nu} \varphi \partial_{\nu} v, \partial_{\nu} v\right)\right|_{\partial M}
\end{aligned}
$$

by using $(A B v, v)-(B v, A v)=-\left.2 i h^{3}\left(\partial_{\nu} \varphi \partial_{\nu} v, \partial_{\nu} v\right)\right|_{\partial M}$ and $(B u, v)=(u, B v)$ since $v$ vanishes at the boundary. For the proof of the first equality we use the Green's identity and for the second, we use the formula (2.1). The proof then proceeds exactly the same way as before, by bounding the extra $X$ factor in the equation and using the positivity of $i([A, B] v, v)$.

Finally, let us recast the inequality $(5.6)$ in the following form, by letting $u=e^{-\frac{\varphi}{h}} v$ and noticing that on $\partial M$ we have $\partial_{\nu} u=e^{-\frac{\varphi}{h}} \partial_{\nu} v$, since $v \in H_{0}^{1}(M)$ :

$$
\begin{aligned}
\left\|e^{\frac{\varphi}{h}} u\right\|_{H_{s c l}^{1}\left(M ; \mathbb{C}^{m}\right)}+\sqrt{h} \| & \sqrt{-\partial_{\nu} \varphi} e^{\frac{\varphi}{h}} \partial_{\nu} u \|_{L^{2}\left(\partial M_{-} ; \mathbb{C}^{m}\right)} \\
& \lesssim h\left\|e^{\frac{\varphi}{h}}(\Delta+X+q) u\right\|_{L^{2}\left(M ; \mathbb{C}^{m}\right)}+\sqrt{h}\left\|\sqrt{\partial_{\nu} \varphi} e^{\frac{\varphi}{h}} \partial_{\nu} u\right\|_{L^{2}\left(\partial M_{+} ; \mathbb{C}^{m}\right)}
\end{aligned}
$$

where we use the notation $\partial M_{ \pm}=\left\{x \in \partial M \mid \pm \partial_{\nu} \varphi(x) \geq 0\right\}$. By generalising appropriately, we have a version of this inequality for an arbitrary vector bundle on $M$.

Now we turn to the proof of inequalities similar to the ones from Theorem 5.2, but with shifted indices of the Sobolev spaces, which is actually necessary to obtain the wanted solvability estimates. This is done using the semiclassical pesudodifferential calculus (see Section 2.3).

Before we start, let us briefly introduce the Sobolev spaces for a real parameter, in a coordinate invariant way. This is described in more detail in [5]. It is a known fact that the connection Laplacian on a compact Riemannian manifold (without boundary) is essentially self-adjoint on the dense subspace $C^{\infty}(N ; E) \subset L^{2}(N ; E)$ (more generally, this holds for any elliptic differential operator on $E$ ), meaning that the closure of $\mathcal{L}_{A}$ is equal to the adjoint $\mathcal{L}_{A}^{*}$.

Then by applying the spectral theorem for unbounded densely defined operators and since $\mathcal{L}_{A}$ is positive, we can define the semiclassical Bessel potentials $J_{A}^{s}=\left(1-h^{2} \Delta_{A}\right)^{\frac{s}{2}}$ for $s \in \mathbb{R}$ (here $\left.\Delta_{A}=-\mathcal{L}_{A}\right)$. The functional calculus from the spectral theorem also gives us that $J_{A}^{s} J_{A}^{t}=J_{A}^{s+t}$ 
and $J_{A}^{s}$ commutes with any function of the connection Laplacian $\mathcal{L}_{A}$. Moreover, it is well-known that a function of a semiclassical PDO is again a semiclassical PDO (see Chapter 8 in [17]); thus $J_{A}^{s}$ is a semiclassical PDO of order $s$. Finally, we define the semiclassical Sobolev spaces $H_{s c l}^{s}$ as the completion of the $C^{\infty}(N ; E)$ in the norm given by:

$$
\|u\|_{H_{s c l}^{s}(N ; E)}=\left\|J_{A}^{s} u\right\|_{L^{2}(N ; E)}
$$

One can easily check that the dual of $H_{s c l}^{s}(N ; E)$ may be isometrically identified with the $H_{s c l}^{-s}(N ; E)$. Similarly, we may define the usual semiclassical Sobolev space, by introducing the semiclassical Bessel potentials $J^{s}=\left(1-h^{2} \Delta\right)^{\frac{s}{2}}$ which define the spaces $H_{s c l}^{s}(N)$; we extend $J^{s}$ to act diagonally on $C^{\infty}\left(N ; \mathbb{C}^{m}\right)$.

Next, observe that we have the following commutator estimates for sections of $E$. Let $\psi$, $\chi \in C_{c}^{\infty}(N)$ with $\chi=1$ near $\operatorname{supp}(\psi)$ and consider any $s, \alpha, \beta \in \mathbb{R}$, and $K \in \mathbb{N}$ - then we can find $C_{K}>0$ such that:

$$
\left\|(1-\chi) J_{A}^{s}(\psi u)\right\|_{H_{s c l}^{\alpha}(N ; E)} \leq C_{K} h^{K}\|u\|_{H_{s c l}^{\beta}(N ; E)}
$$

This follows from the pseudolocality of the semiclassical PDOs (see (2.17) ) and the mapping properties of semiclassical PDOs on Sobolev spaces (see 2.14). Moreover, we record another commutator estimate:

$$
\left\|\left[D, J_{A}^{s}\right] u\right\|_{L^{2}(N ; E)} \leq C h\|u\|_{H_{s c l}^{s}(N ; E)}
$$

where $D$ is a first order, diagonal semiclassical differential operator in $E$ over $N$; this follows from the formula for the symbol of the commutator of two semiclassical PDOs (see (3.1)).

For what follows, assume that the $\mathrm{LCW} \varphi$ is a smooth function in a neighbourhood of $\bar{U}$ and extend this function smoothly to $N$. We are now ready to shift the indices of the Sobolev estimates from Theorem 5.2 .

THEOREM 5.4. Under the assumptions of Theorem 5.2 and given $s \in \mathbb{R}$, there exist constants $C_{s}$ and $h_{s}>0$ such that for all $0<h \leq h_{s}$ and $u \in C_{c}^{\infty}\left(M^{\text {int }} ; \mathbb{C}^{m}\right)$ :

$$
\left\|e^{\frac{\varphi}{h}} u\right\|_{H_{s c l}^{s+1}\left(N ; \mathbb{C}^{m}\right)} \leq C_{s} h\left\|e^{\frac{\varphi}{h}}(\Delta+X+q) u\right\|_{H_{s c l}^{s}\left(N ; \mathbb{C}^{m}\right)}
$$

Moreover, there are corresponding constants such that for every $u \in C_{c}^{\infty}\left(M^{\text {int }} ; E\right)$ :

$$
\left\|e^{\frac{\varphi}{h}} u\right\|_{H_{s c l}^{s+1}(N ; E)} \leq C_{s}^{\prime} h\left\|e^{\frac{\varphi}{h}} \mathcal{L}_{A, Q} u\right\|_{H_{s c l}^{s}(N ; E)}
$$

Proof. We closely follow the proof of Lemma 4.3 from $\left[\mathbf{2 1}\right.$. Let us introduce $P_{\varphi_{\epsilon}}=$ $e^{\frac{\varphi_{\epsilon}}{h}} h^{2}(\Delta+X+q) e^{-\frac{\varphi_{\epsilon}}{h}}$ and let $\chi \in C_{0}^{\infty}(U)$ such that $\chi=1$ near $M$; here $\varphi_{\epsilon}$ comes from the proof of Theorem 5.2. Then we have by (5.5) and (5.8):

$$
\begin{aligned}
h\|u\|_{H_{s c l}^{s+1}} & \leq h\left\|\chi J^{s} u\right\|_{H_{s c l}^{1}}+h\left\|(1-\chi) J^{s} u\right\|_{H_{s c l}^{1}} \\
& \lesssim \sqrt{\epsilon}\left\|P_{\varphi_{\epsilon}}\left(\chi J^{s} u\right)\right\|_{L^{2}}+h^{2}\|u\|_{H_{s c l}^{s+1}}
\end{aligned}
$$

which means that the second term may be absorbed to the left hand side for small $h$. Furthermore, for some $\chi^{\prime} \in C_{0}^{\infty}(U)$ with $\chi^{\prime}=0$ near $M$, by (5.5) again:

$$
\left\|\left[P_{\varphi_{\epsilon}}, \chi\right] J^{s} u\right\|_{L^{2}}=\left\|\left[P_{\varphi_{\epsilon}}, \chi\right] \chi^{\prime} J^{s} u\right\|_{L^{2}} \lesssim\left\|\chi^{\prime} J^{s} u\right\|_{H_{s c l}^{1}} \lesssim h^{2}\|u\|_{H_{s c l}^{s+1}}
$$

so after absorbing the remaining factors, we have:

$$
h\|u\|_{H_{s c l}^{s+1}} \lesssim \sqrt{\epsilon}\left\|J^{s} P_{\varphi_{\epsilon}} u\right\|_{L^{2}}+\sqrt{\epsilon}\left\|\left[P_{\varphi_{\epsilon}}, J^{s}\right] u\right\|_{L^{2}}
$$


The first term gives the right bound; for the second one, by expanding the operator and putting $X_{\varphi_{\epsilon}}=e^{\frac{\varphi_{\epsilon}}{h}} X e^{-\frac{\varphi_{\epsilon}}{h}}$, we have:

$$
P_{\varphi_{\epsilon}}=h^{2} \Delta-\left|d \varphi_{\epsilon}\right|^{2}+2\left\langle d \varphi_{\epsilon}, h d(\cdot)\right\rangle-h \Delta \varphi_{\epsilon}+h^{2} X_{\varphi_{\epsilon}}+h^{2} q=: h^{2} \Delta+P_{1}
$$

Since $\left[h^{2} \Delta, J^{s}\right]=0$ and since $J^{s}$ acts diagonally, by the composition formula we have $\left[J^{s}, P_{1}\right]=$ $h R_{1}$ where $R_{1}$ a semiclassical PDO of order $s$. Thus by taking $\epsilon$ to be small enough (and such that $\left.h \leq \epsilon \epsilon_{0}\right)$, we may absorb this remainder to the left hand side.

For an arbitrary vector bundle, note that all the steps above work the same with $J_{A}^{s}$ instead of $J^{s}$, until the estimate for $\left\|\left[P_{\varphi_{\epsilon}}, J_{A}^{s}\right] u\right\|_{L^{2}}$. In local coordinates, we have the expansion

$$
e^{\frac{\varphi_{\epsilon}}{h}} h^{2} \mathcal{L}_{A, Q} e^{-\frac{\varphi_{\epsilon}}{h}}=h^{2} \mathcal{L}_{A}-\underbrace{\left(\left|d \varphi_{\epsilon}\right|^{2}-2\left\langle d \varphi_{\epsilon}, h d(\cdot)\right\rangle+h \Delta \varphi_{\epsilon}\right)}_{D}+\underbrace{2 h\left\langle A, d \varphi_{\epsilon}\right\rangle}_{P_{2}}
$$

where $D$ is a diagonal first order semiclassical differential operator. Now observe that $\left[\mathcal{L}_{A}, J_{A}^{s}\right]=$ 0 and also that locally the symbol of $\left[D, J_{A}^{s}\right]$ is in $h S^{s}$, and so is the symbol of $\left[P_{2}, J_{A}^{s}\right]$. This implies that $\left[-D+P_{2}, J_{A}^{s}\right]$ is in $h \Psi^{s}(M)$, which makes us able to absorb the extra factor for small enough $\epsilon$ and finish the proof.

Essentially the only case that we will use in the previous theorem is the case $s=-1$; it appears that it is necessary in the following result, to establish the existence of an $H^{1}$ solution to our equation with a suitable norm estimate (otherwise, with Theorem 5.2 we would only get solutions in $L^{2}$ with bounds in $H^{-1}$ norm). It is left without a proof, since it is well-known and formally follows from the scalar case in Theorem 4.4 in [21].

THEOREM 5.5. Given a connection $A$ and an endomorphism $Q$ of $E$, there exists a positive constant $h_{0}$ such that for any $0<h \leq h_{0}$ and any section $f \in L^{2}(M ; E)$, there exists a solution $u \in H^{1}(M ; E)$ to the equation $e^{\frac{\varphi}{h}} \mathcal{L}_{g, A, Q} e^{-\frac{\varphi}{h}} u=f$ satisfying:

$$
\|u\|_{H_{s c l}^{1}(M ; E)} \leq C h\|f\|_{L^{2}(M ; E)}
$$





\section{CHAPTER 6}

\section{The non-abelian ray transform reduction and simple manifolds}

In this chapter, we construct the special CGO solutions of the form $u=e^{-\frac{\Psi}{h}}(a+r)$ (for suitable $\Psi, a$ and $r$ - see the introduction for more background on this) to the connection Laplacian equation $\mathcal{L}_{A}(u)=0$, in the particular case when the transversal manifold is simple. In this case, we have an easy ansatz to the transport and the eikonal equation, so we get away without using the construction of Gaussian Beams in Chapter 4. The purpose of this is to reduce Conjecture $\mathrm{A}$ in this case to a new non-abelian ray transform - see Question 6.11 below.

\subsection{The CGO construction for simple transversal manifolds}

Throughout the chapter, we will be working in the following setting: $M$ is an $n$-dimensional compact manifold with boundary, $E=M \times \mathbb{C}^{m}$ is the trivial vector bundle of rank $m$ with the standard fibrewise Hermitian inner product, $A$ a unitary connection on it and $Q$ an $m$ by $m$ matrix potential (section of $\operatorname{End}(E)$ ). Furthermore, our assumption will be that $M_{0}$ is simple and that $M$ is isometrically embedded inside the manifold of the same dimension $\mathbb{R} \times M_{0}$, with the product metric $g=e \oplus g_{0}$.

Recall that the manifold $M_{0}$ is simple if the exponential map $\exp _{p}: \exp _{p}^{-1}\left(M_{0}\right) \rightarrow M_{0}$ is a diffeomorphism for every point $p \in M_{0}$ and the boundary $\partial M$ is strictly convex. Simplicity of $M_{0}$ is a natural assumption and many questions about the X-ray transform are posed in this setting (see the introduction for more details).

We start with stating an identity which will be useful for identifying different parts of the CGO solution. The proof is left as an exercise.

Lemma 6.1. The following identity holds, for $s \in \mathbb{C}, \rho$ a smooth function on $M, u$ a section of $E, X$ a smooth $m \times m$ matrix with entries as vector fields and $q$ a smooth $m \times m$ matrix potential:

$$
e^{-s \rho}(\Delta+X+q) e^{s \rho} u=(\Delta+X+q) u+s((\Delta \rho) u+X(\rho) u-2\langle\nabla \rho, \nabla u\rangle)-s^{2}|d \rho|^{2} u
$$

Now plugging in the specific form of the solution as above $u=e^{-\frac{\Psi}{h}}(a+r)$ to the equation $h^{2} \mathcal{L}_{A, Q} u=0$ ( $a$ and $r$ are $\mathbb{C}^{m}$-valued, $\Psi$ a complex function) and using Lemma 6.1, we get three equations:

$$
\begin{aligned}
|d \Psi|^{2} & =0 \\
-2\langle d \Psi, d a\rangle+(\Delta \Psi) a+X(\Psi) a & =0 \\
e^{\frac{\Psi}{h}} \mathcal{L}_{A, Q} e^{-\frac{\Psi}{h}} r & =-\mathcal{L}_{A, Q} a
\end{aligned}
$$

where the first two of the them correspond to the dominating factors (the coefficients next to $h^{0}$ and $h^{1}$, respectively) when $h \rightarrow 0$ and the last one makes sure we get an exact solution and solves for the residue. The notation $\langle d \Psi, d a\rangle$ means that we consider the vector formed by taking the inner product of each component of $d a$ with $d \Psi$. Recall that $X=-2 g^{i j} A_{i} \frac{\partial}{\partial x^{j}}$ is derived in 2.8 from the pair $(A, Q)$. 
6.1.1. Eikonal equation. This is the equation 6.1 above. Recall that in this case the operation $|\cdot|$ is just a complex bilinear form obtained by extending the Riemannian real inner product. Thus, if we write $\Psi=\varphi+i \psi$, the equation can be rewritten as:

$$
|\nabla \psi|^{2}=|\nabla \varphi|^{2}, \quad\langle\nabla \psi, \nabla \varphi\rangle=0
$$

Here we let $\varphi$ to be the LCW given by $\varphi(x)=x_{1}$. With this special choice for $\varphi$, our equations become simple:

$$
|\nabla \psi|=1, \quad \frac{\partial \psi}{\partial x_{1}}=0
$$

because of the splitting of the metric in $\mathbb{R} \times M_{0}$. Here we will fix a polar coordinate system: we pick a point $\omega \in M_{0}$ such that $\left(x_{1}, \omega\right)$ is not in $M$ for any $x_{1}$. We can always do this if we enlarge $M_{0}$ slightly at the beginning, keeping the metric simple (this is always possible see [21]), to some manifold $D$ such that:

$$
(M, g) \Subset\left(\mathbb{R} \times M_{0}, g\right) \Subset(\mathbb{R} \times D, g)
$$

We then use the geodesic polar coordinate system to get a coordinate chart $\left(x_{1}, r, \theta\right)$ for $\theta \in S^{n-2}$, to cover $\mathbb{R} \times M_{0}$, in which the metric has a nice form.

One can then check that $\psi=r$ solves $(6.5)$ and in this case $\Psi=x_{1}+i r$ (note that the solution depends on $\omega$ ). Observe that we could have chosen $\varphi=-x_{1}$, in which case $\Psi=-x_{1}+i r$ works equally well. This will be useful when we plug the solutions into our identity in Theorem 2.6, so that the exponential parts cancel in the product.

6.1.2. Transport equation. This is the equation 6.2 . We now proceed to the calculation of the three terms in this equation, taking $\Psi=x_{1}+i r$ for the solution of the eikonal equation. We get the expressions:

$$
\begin{gathered}
\langle d \Psi, d a\rangle=\frac{\partial \Psi}{\partial x_{1}} \frac{\partial a}{\partial x_{1}}+\sum_{j, k \geq 2} g^{j k} \frac{\partial \Psi}{\partial x^{j}} \frac{\partial a}{\partial x^{k}}=\left(\frac{\partial}{\partial x_{1}}+i \frac{\partial}{\partial r}\right) a \\
\Delta \Psi=-|g|^{-1 / 2}\left(\sum_{j, k \geq 1} \frac{\partial}{\partial x^{j}}\left(|g|^{1 / 2} g^{j k} \frac{\partial \Psi}{\partial x^{k}}\right)\right)=-|g|^{-1 / 2}\left(\frac{\partial}{\partial x_{1}}+i \frac{\partial}{\partial r}\right)\left(|g|^{1 / 2}\right) \\
X(\Psi)=-2\left(\sum_{j, k \geq 1} g^{j k} A_{j} \frac{\partial}{\partial x^{k}}\left(x_{1}+i r\right)\right)=-2\left(A_{1}+i A_{r}\right)
\end{gathered}
$$

Here $A_{1}$ and $A_{r}$ are the $d x_{1}$ and $d r$ components of $A$, respectively and we are taking the $\left(x^{2}, \ldots, x^{n}\right)$ coordinates on $M_{0}$, where $x^{2}=r$. We set $z=x_{1}+i r$ and so we define the complex derivatives as $\frac{\partial}{\partial \bar{z}}=\frac{1}{2}\left(\frac{\partial}{\partial x_{1}}+i \frac{\partial}{\partial r}\right)$ and $\frac{\partial}{\partial z}=\frac{1}{2}\left(\frac{\partial}{\partial x_{1}}-i \frac{\partial}{\partial r}\right)$. Then the equation 6.2 takes the form:

$$
4 \frac{\partial a}{\partial \bar{z}}+2|g|^{-1 / 2} \frac{\partial}{\partial \bar{z}}\left(|g|^{1 / 2}\right) a+2\left(A_{1}+i A_{r}\right) a=0
$$

By introducing an integrating factor and using the substitution $b=a|g|^{1 / 4}$, we get the following nicer form:

$$
\frac{\partial b}{\partial \bar{z}}=-\frac{1}{2}\left(A_{1}+i A_{r}\right) b
$$

Analogously, using the other solution $\Psi=-x_{1}+i r$ of the eikonal equation, we get:

$$
\frac{\partial b}{\partial z}=\frac{1}{2}\left(-A_{1}+i A_{r}\right) b
$$

Since (6.8) can be obtained from 6.7) by conjugation, we will focus only on the latter. Actually we consider a slightly more general equation:

$$
\frac{\partial C}{\partial \bar{z}}=B C
$$


where $C\left(\theta, x_{1}, r\right)$ is a smooth $m$ by $m$ matrix function and we denoted $B=-\frac{1}{2}\left(A_{1}+i A_{r}\right)$. We impose one additional condition that $C$ should be invertible. Such a matrix $C$ will play an important role and we will need the solution on an open bounded subset of the plane, depending smoothly on $\theta$.

If one is interested in solving this equation on the whole domain of $\mathbb{C}$, a natural boundary condition would be to have $C$ approaching the identity at $\infty$; however this might be impossible - see 26 for the proof of existence of a $C$ which has polynomial growth.

For $m=1$, we may solve 6.9 by substituting the exponential function $C=e^{\Phi}$ and then using the Cauchy operator to solve $\bar{\partial} \Phi=B \rrbracket^{1}$ However, for $m>1$ the situation complicates, so we give one proof of existence in the next subsection and a brief overview of other approaches. Given a matrix $C$ solution of (6.9), one solution of the transport equation (6.7) is given by $a=C h$, where $h$ is holomorphic in each coordinate.

\subsubsection{Complex geometric approach to the construction of the solution to trans-} port equation. Using some standard theory of holomorphic vector bundles one can describe a solution to the transport equation $(6.9)$ in a geometric way. References are books by Kobayashi 42 (Propositon 3.7) and Foster $\mathbf{3 2}$ (Theorem 30.1).

THeOREM 6.2. Let $E$ be a $C^{\infty}$ complex vector bundle over a complex manifold $M$. Then if $D$ is a connection on $E$ such that $D^{\prime \prime} \circ D^{\prime \prime}=0$, then there exists a unique holomorphic vector bundle structure on $E$ such that $D^{\prime \prime}=d^{\prime \prime}$.

THEOREM 6.3. Let $X$ be an open Riemann surface and $E$ a holomorphic vector bundle over $X$, of rank $m$. Then $E$ is trivial, i.e. there exists a set of holomorphic sections $s_{i}, i=1, \ldots, m$ such that they span $E_{p}$ for each point $p$ in $X$.

In the former theorem, by $D^{\prime \prime}$ we mean the $(0,1)$ component of the connection derivative and by $d^{\prime \prime}=\bar{\partial}$ the $(0,1)$ component of the exterior derivative.

Theorem 6.4. Let $\Omega \subset \mathbb{C}$ be an open subset of the complex plane and let $E=\Omega \times \mathbb{C}^{m}$, equipped with a connection $D$. Then there exists a smoothly varying invertible matrix $F$ such that $\bar{\partial} F=-F A_{0,1}$, where $A_{0,1}$ is the $(0,1)$ part of the connection matrix of $D$. In particular, for any matrix $B$, there exists an invertible, smoothly varying matrix $C$ such that $\frac{\partial C}{\partial \bar{z}}=B C$.

Proof. The proof relies on the previous two theorems; namely, we automatically have $D^{\prime \prime} \circ D^{\prime \prime}=0$ by dimension. Thus, there exists a holomorphic structure on $E$ such that $D^{\prime \prime}=d^{\prime \prime}$. Although our vector bundle is smoothly trivial, we do not know if it is holomorphically trivial - this is given by Theorem 6.3. Thus, there exists a set of holomorphic trivialisations $s_{i}$, $i=1, \ldots, m$ such that they are linearly independent at each point of $\Omega$; in these new coordinates, we also have $D^{\prime \prime}=d^{\prime \prime}$. In other words, there exists a smoothly (not necessarily holomorphically) varying matrix $F: \Omega \rightarrow G L(m, \mathbb{C})$ such that, $s_{i}=F e_{i}$, where $e_{i}$ is our standard global frame of $E$. Then we have the change of basis law for connections:

$$
0=\bar{\partial} s_{i}=D^{\prime \prime} s_{i}=D^{\prime \prime}\left(F e_{i}\right)=\bar{\partial} F e_{i}+F D^{\prime \prime} e_{i}=\bar{\partial} F e_{i}+F A_{0,1} e_{i}
$$

for all $i=1, \ldots, m$. Thus we get, in matrix form:

$$
\bar{\partial} F=-F A_{0,1}
$$

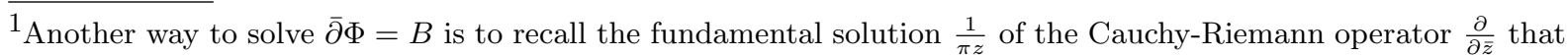
satisfies $\frac{\partial}{\partial \bar{z}} \frac{1}{\pi z}=\delta$, where $\delta$ is the Dirac delta; then the convolution $\Phi=\frac{1}{\pi z} * B$ is a solution of $\frac{\partial}{\partial \bar{z}} \Phi=B$ (here $B$ has compact support). This is just a restatement of the generalised Cauchy integral formula that is being referred to in the text, which gives: $\Phi(\omega)=\frac{1}{2 \pi i} \int_{\mathbb{C}} \frac{B(z)}{z-\omega} d z d \bar{z}$.
} 
By picking the $(0,1)$ part of the connection matrix to be $B d \bar{z}$, and letting $C=F^{-1}$, we get $\frac{\partial C}{\partial \bar{z}}=B C$.

REMARK 6.5. We digress slightly to note that there are examples of smoothly trivial holomorphic line bundles, but not holomorphically trivial. The long exact sequence associated to the short exact sequence $0 \rightarrow 2 \pi i \mathbb{Z} \rightarrow \mathcal{O} \rightarrow \mathcal{O}^{*} \rightarrow 0$ (here $\mathcal{O}$ and $\mathcal{O}^{*}$ are the sheaves of holomorphic and nowhere vanishing holomorphic functions, respectively) that the map $c_{1}: \operatorname{Pic}(M) \rightarrow \mathbb{Z}$ given by the first Chern class has a non-trivial kernel over a surface of positive genus $M(\operatorname{Pic}(M)$ is the holomorphic Picard group).

Theorem 6.4 provides us with a geometric interpretation of $(6.9)$ for a fixed $\theta$. In order to solve this equation smoothly in $\theta$, we need to go through the proof of trivialising a family of holomorphic vector bundles parametrically. We will not do this here, since there are already a few proofs of existence of such parametric solutions present in other sources.

Let us give a brief overview of proofs of existence of (invertible) solutions to the above equation we found in literature. As mentioned, Eskin [26] gives us $C$ depending smoothly on a parameter, with polynomial growth as $|z| \rightarrow \infty$. A more concise proof is given by the same author and Ralston in Theorem 4, $[\mathbf{2 9}]\left(Y=S^{n-2}\right.$ in our case) - it relies on solving the equation locally in $z$ using the Cauchy operator to transform it to an integral equation and then gluing these local solutions together using the Cartan's lemma. Finally, Nakamura and Uhlmann [57 also provide us with another method.

6.1.4. The inhomogeneous part. Here we deal with the third equation set out above, the equation (6.3). With the Carleman estimates established so far, we can easily construct the residue with the wanted estimates - we just use Theorem 5.5 to solve for the $h$-dependent residue $r_{h}$ (note the distinction between the radial variable $r$ and the function $r_{h}$ ), such that $\left\|r_{h}\right\|_{L^{2}(M ; E)}=O(h)$ and $\left\|r_{h}\right\|_{H^{1}(M ; E)}=O(1)$; equivalently $\left\|r_{h}\right\|_{H_{s c l}^{1}(M ; E)}=O(h)$.

\subsection{Consequences of the CGO construction and recovering the connection}

In this section, we use the previously obtained CGO solutions to deduce some new information from the equality of the DN maps. Reducing to an X-ray transform or asking for injectivity of some other transform is often the way to make the final step in solving inverse problems: see 14, 21, 23, 64 for examples of such results for the X-ray transform or $2 \mathbf{2 2}$ for an example of the Radon transform on planes; this is the viewpoint we will take.

We equip $E=M \times \mathbb{C}^{m}$ with two potentials $Q_{1,2}$ and unitary connections $A_{1,2}$; we assume that $\Lambda_{A_{1}, Q_{1}}=\Lambda_{A_{2}, Q_{2}}$. It is technically easier to consider the endomorphism bundle $E^{\prime}=M \times \mathbb{C}^{m \times m}$ and extend the action of $\mathcal{L}_{A_{1}, Q_{1}}$ and $\mathcal{L}_{A_{2}, Q_{2}}$ in the trivial way to sections of $E^{\prime}$ (by matrix multiplication). So we consider matrix solutions $U_{1}$ and $U_{2}$ to $\mathcal{L}_{A_{1}, Q_{1}} U_{1}=0$ and $\mathcal{L}_{A_{2}, Q_{2}^{*}} U_{2}=0$, constructed by our work in previous subsections, which are of the form:

$$
\begin{aligned}
& U_{1}=e^{-\frac{x_{1}+i r}{h}}\left(|g|^{-1 / 4} C_{1} H\left(x_{1}, r\right) b(\theta)+R_{1}\right) \\
& U_{2}=e^{-\frac{-x_{1}+i r}{h}}\left(|g|^{-1 / 4} C_{2}+R_{2}\right)
\end{aligned}
$$

where $H$ a holomorphic matrix, $b$ is a smooth function and we have the estimates $\left\|R_{1}\right\|_{H_{s c l}^{1}\left(M ; E^{\prime}\right)}=$ $O(h)$ and $\left\|R_{2}\right\|_{H_{s c l}^{1}\left(M ; E^{\prime}\right)}=O(h)$. The invertible matrices $C_{i}$ are given by solving the transport equations 6.7 and 6.8 in the matrix form:

$$
\frac{\partial C_{1}}{\partial \bar{z}}=-\frac{1}{2}\left(\left(A_{1}\right)_{1}+i\left(A_{1}\right)_{r}\right) C_{1} \quad \text { and } \quad \frac{\partial C_{2}}{\partial z}=\frac{1}{2}\left(-\left(A_{2}\right)_{1}+i\left(A_{2}\right)_{r}\right) C_{2}
$$


We wish to plug these in the identity obtained in Theorem 2.7. Note that we have:

$$
\begin{aligned}
& d U_{1}=e^{-\frac{x_{1}+i r}{h}}\left(-\frac{d x_{1}+i d r}{h}\left(|g|^{-\frac{1}{4}} C_{1} H b+R_{1}\right)+d\left(|g|^{-\frac{1}{4}} C_{1} H b\right)+d\left(R_{1}\right)\right) \\
& d U_{2}^{*}=e^{\frac{x_{1}+i r}{h}}\left(\frac{d x_{1}+i d r}{h}\left(|g|^{-\frac{1}{4}} C_{2}^{*}+R_{2}^{*}\right)+d\left(|g|^{-\frac{1}{4}} C_{2}^{*}\right)+d\left(R_{2}^{*}\right)\right)
\end{aligned}
$$

Therefore, in the limit $h \rightarrow 0$, for $\tilde{A}=A_{2}-A_{1}$ :

$$
\lim _{h \rightarrow 0} h\left(U_{1}\left(d U_{2}^{*}\right)-\left(d U_{1}\right) U_{2}^{*}, \tilde{A}\right)_{M}=-2 \int_{M} \operatorname{tr}\left(|g|^{-\frac{1}{2}} b(\theta) C_{1} H C_{2}^{*}\left(\tilde{A}_{1}+i \tilde{A}_{r}\right)\right) d V_{g}
$$

by using Cauchy-Schwartz and the bounds we have on the $\left\|R_{1}\right\|_{H_{s c l}^{1}}$ and $\left\|R_{2}\right\|_{H_{s c l}^{1}}$, along with the fact that everything else is uniformly bounded. Moreover, since the $A_{i}$ and $Q_{i}$ are bounded for $i=1,2$ and the exponential parts of $U_{1}$ and $U_{2}^{*}$ cancel, the first integral in the identity is equal to $O(1)$. Thus we get, by taking the limit $h \rightarrow 0$ :

$$
\int_{M}|g|^{-\frac{1}{2}} b(\theta) \operatorname{tr}\left(C_{1} H C_{2}^{*}\left(\tilde{A}_{1}+i \tilde{A}_{r}\right)\right) d V_{g}=0
$$

where $d V_{g}$ is the volume form. Since $d V_{g}=|g|^{1 / 2} d x_{1} d r d \theta$ and since we can vary $b$ so that it approximates the delta function $\delta_{\eta}$ for some fixed angle $\eta$, by rearranging the terms in the trace bracket we obtain:

$$
\int_{M_{0}^{\eta}} \operatorname{tr}\left(H C_{2}^{*}\left(\tilde{A}_{1}+i \tilde{A}_{r}\right) C_{1}\right) d z \wedge d \bar{z}=0
$$

where $z=x_{1}+i r$ and $M_{0}^{\eta}:=[-N, N] \times M_{0} \cap\{\theta=\eta\}$, for some large $N$ (we also have $M^{\eta}=M \cap\{\theta=\eta\}$ is a 2-dimensional smooth manifold for almost all $\eta$ by Sard's theorem; the previous integral can be made over such $M^{\eta}$, too), such that $[-N, N] \times D$ contains a neighbourhood of $M$.

Here, we extended the connections $A_{1}$ and $A_{2}$ to the outside of $M$ (whole of $\mathbb{R} \times M_{0}$ ), such that they are unitary, compactly supported and such that $A_{1}=A_{2}$ outside $M$. This is allowed by Theorem 3.4, which gives us that the full jets of $A_{1}$ and $A_{2}$ are the same in suitable gauges. Therefore, by applying the gauge from Theorem 3.4 at the start of our analysis, we may assume that $A_{1}$ and $A_{2}$ are extended as stated.

Now, by using the equations 6.12 , we get:

$$
\frac{\partial}{\partial \bar{z}}\left(C_{2}^{*} C_{1}\right)=\frac{1}{2} C_{2}^{*}\left(\tilde{A}_{1}+i \tilde{A}_{r}\right) C_{1}
$$

where we also used that $A_{i}$ s are skew-Hermitian. By substituting $C_{0} H$ in place of $H$ in the identity (6.14), where $C_{0}$ is a constant matrix and $H$ holomorphic, and by varying the entries of $C_{0}$ we obtain:

$$
\int_{M_{0}^{\eta}} H \frac{\partial}{\partial \bar{z}}\left(C_{2}^{*} C_{1}\right) d z \wedge d \bar{z}=0
$$

and therefore by Stokes' theorem, we get:

$$
\int_{\partial M_{0}^{\eta}} H C_{2}^{*} C_{1} d z=0
$$

Note that $H$ is an arbitrary holomorphic matrix, i.e. $\frac{\partial H}{\partial \bar{z}}=0$ and that the order in which we take matrix multiplication inside the integral is important.

We would now like to deduce a suitable transport equation on $\mathbb{R} \times S M_{0}$ and try to solve the problem from there.

Recall from Section 6.1.1 the enlarged simple manifold $D$, which contains $M_{0}$. As we go along $\partial D$ and follow the tangent vectors, we obtain families of geodesics on $M_{0}$. Let us denote by $C_{1}\left(p, \theta, x_{1}, r\right)$ and $C_{2}\left(p, \theta, x_{1}, r\right)$ the solutions to equations 6.12$)$, where $p$ denotes the point 


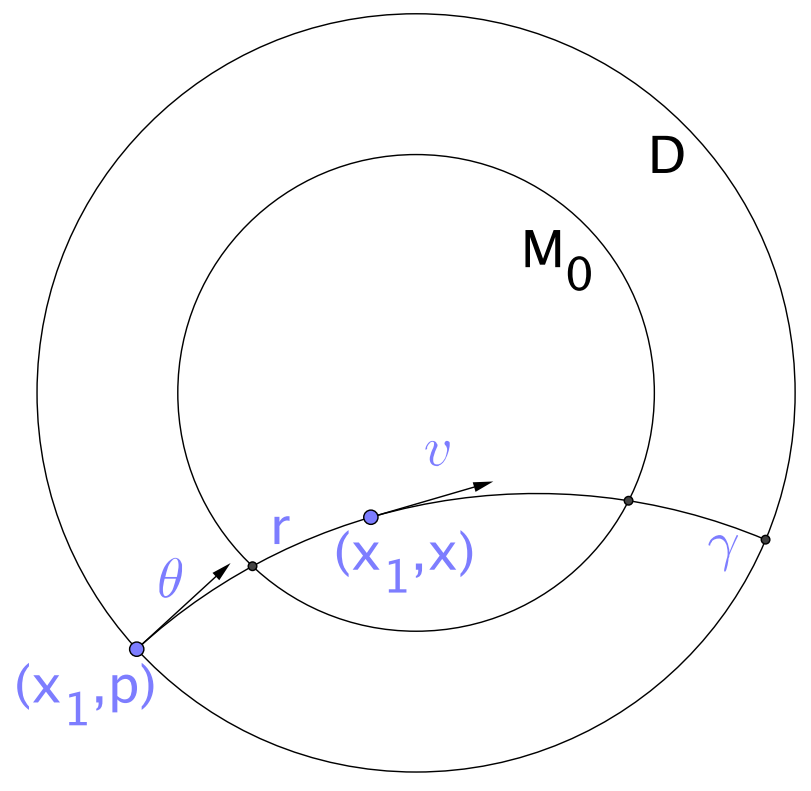

FiguRE 1. The construction of the matrix function $G\left(x_{1}, x, v\right)$ : we are following the geodesic $\gamma$ at $\left(x_{1}, x\right)$ in the direction $-v$ up to the point $\left(x_{1}, p\right)$ on $\partial D$. The angle $\theta$ denotes the polar coordinate of the point $x$ with centre at $p$; distance between $x$ and $p$ is $r$.

of the origin of the polar coordinate system. As explained previously in Subsection 6.1.3, we may construct solutions to 6.12 depending smoothly on a parameter, giving $C_{1}$ and $C_{2}$ smooth as we vary $\left(p, \theta, x_{1}, r\right)$.

Now given any $\left(x_{1}, x\right) \in \mathbb{R} \times M_{0}$ and $v \in S_{x} M_{0}$ a unit tangent vector, we may trace backwards the geodesic $\gamma$ starting at $\left(x_{1}, x\right)$ with speed $v$ (or go forwards in time with the geodesic with speed $-v)$, until we hit $\partial D$; call this point $\left(x_{1}, p\right)$ - see Figure1. Since $D$ is simple, we have the smooth dependence $p=p(x, v)$. Define

$$
G\left(x_{1}, x, v\right)=C_{1}\left(p, \theta, x_{1}, r\right) C_{2}^{*}\left(p, \theta, x_{1}, r\right)
$$

where $r$ is the length along $\gamma$ from $\left(x_{1}, p\right)$ to $\left(x_{1}, x\right), \theta$ is the coordinate of $\left(x_{1}, x\right)$ in the polar coordinate system (i.e. $\dot{\gamma}$ at the point $\left(x_{1}, p\right)$ ). Again since $D$ is simple we have the smooth dependence $\theta=\theta(x, v)$, which implies that $G$ is smooth. Therefore, we obtain a smooth matrix function $G$ (section of $E^{\prime}$ ) on $\mathbb{R} \times S M_{0}$, where $S M_{0}$ denotes the unit sphere bundle. By the previous analysis, we have an equation for $G$ :

$$
2 \frac{\partial G}{\partial \bar{z}}=-\left(\left(A_{1}\right)_{1}+i\left(A_{1}\right)_{r}\right) G+G\left(\left(A_{2}\right)_{1}+i\left(A_{2}\right)_{r}\right)
$$

on the planes which are generated by the $x_{1}$ direction and a geodesic, i.e. by setting $\theta$ to be constant for a given $p \in \partial D$. From the previous equation we easily deduce that we have globally:

$$
\left(\frac{\partial}{\partial x_{1}}+i X(x, v)\right) G=-A_{1}\left(\frac{\partial}{\partial x_{1}}+i v\right) G+G A_{2}\left(\frac{\partial}{\partial x_{1}}+i v\right)
$$

for all $x \in M_{0}, v$ unit tangent vectors in $S_{x} M_{0}$ and $x_{1} \in \mathbb{R} ; X(x, v)$ is the geodesic vector field on $S M_{0}$. Let us make a shorthand notation for the complex vector field $\mathbb{X}\left(x_{1}, x, v\right)=\frac{\partial}{\partial x_{1}}+i X(x, v)$.

First of all, let us see what information our integral equation (6.17) gives us. We will need the following standard result: 
Lemma 6.6. Let $\Omega \subset \mathbb{C}$ be a domain with smooth boundary and let $f$ be a smooth function on $\partial \Omega$. Then $f$ is a restriction of a holomorphic function $h$ on $\Omega$, i.e. $f=\left.h\right|_{\Omega}$ if and only if

$$
\int_{\partial \Omega} g(z) f(z) d z=0
$$

for all holomorphic functions $g$ on $\Omega$, which have a continuous extension to $\bar{\Omega}$.

The proof of this Lemma uses the Plemelj-Sokhotski-Privalov formula and it follows from the proof of Lemma 5.1 in $[\mathbf{2 2}]$. As an application of this result, we have:

Lemma 6.7. There exists a holomorphic, invertible matrix function $F$ on $M_{0}^{\eta}$, such that $\left.F^{-1}\right|_{\partial M_{0}^{\eta}}=\left.C_{2}^{*} C_{1}\right|_{\partial M_{0}^{\eta}}$.

Proof. By applying Lemma 6.6 to the equation (6.17), we deduce there exists a holomorphic matrix function $F^{\prime}$, such that $\left.F^{\prime}\right|_{\partial M_{0}^{\eta}}=\left.C_{2}^{*} C_{1}\right|_{\partial M_{0}^{\eta}}$. We need to prove $F^{\prime}$ is invertible on $M_{0}^{\eta}$.

Firstly, $M_{0}^{\eta}=[-N, N] \times[0, L]$ in local coordinates $(L$ is the length of the segment of the unit speed geodesic starting at a point $p \in \partial D$, which lies in $M_{0}$ ), which is simply-connected. Therefore, since $\operatorname{det}\left(C_{1}\right) \neq 0$ on $M_{0}^{\eta}$, it is a standard fact that $\operatorname{det}\left(C_{1}\right)$ admits a logarithm: we have a smooth function $\Phi_{1}$ on $M_{0}^{\eta}$ such that $\operatorname{det}\left(C_{1}\right)=e^{\Phi_{1}}$ and similarly we have $\Phi_{2}$ such that $\operatorname{det}\left(C_{2}\right)=e^{\Phi_{2}}$. From this, we infer that the variation of the $\operatorname{argument}$ of $\left.\operatorname{det}\left(F^{\prime}\right)\right|_{\partial M_{0}^{\eta}}=$ $\left.e^{\Phi_{1}+\bar{\Phi}_{2}}\right|_{\partial M_{0}^{\eta}}$ is zero, since $\Phi_{1}$ and $\Phi_{2}$ are honest functions. Therefore, by the argument principle applied to the holomorphic function $\operatorname{det}\left(F^{\prime}\right)$, we conclude $F^{\prime}$ is invertible on the whole of $M_{0}^{\eta}$. By setting $F=\left(F^{\prime}\right)^{-1}$, we are done $2^{2}$

More generally, we have such $F$ depending smoothly on the parameters in the influx boundary $(p, \theta) \in \partial_{+} S D$ so we obtain a smooth matrix function $F$ on $\left.[-N, N] \times S M_{0}\right]^{3}$ such that $\left.F\right|_{[-N, N] \times \partial S M_{0}}=\left.\left(C_{2}^{*} C_{1}\right)\right|_{[-N, N] \times \partial S M_{0}}$ and $\mathbb{X}(F)=0$. Then we can redefine the solution $C_{2}$ to equations 6.12) (parametrised by $(p, \theta) \in \partial_{+} S D$ ), by setting $C_{2}^{\prime}=C_{2} F^{*}$. The transport equations will be satisfied again, but more importantly, we must have 6.18 fulfilled with the new $G^{\prime}\left(x_{1}, x, v\right)=C_{1}\left(p, \theta, x_{1}, r\right)\left(C_{2}^{\prime}\right)^{*}\left(p, \theta, x_{1}, r\right)$ defined analogously as before and:

$$
\left.G^{\prime}\right|_{[-N, N] \times \partial S M_{0}}=\left.\left(C_{1} C_{2}^{*}\right)\right|_{[-N, N] \times \partial S M_{0}}=\left.\left(C_{1} F C_{2}^{*}\right)\right|_{[-N, N] \times \partial S M_{0}}=\left.I d\right|_{[-N, N] \times \partial S M_{0}}
$$

by the definition of $F$. Let us relabel the $G^{\prime}$ back to $G$.

Let us now consider a reduction of the problem to a convex region, i.e. a larger manifold with certain properties. We take $M^{\prime}$ to be a slightly smaller manifold than $[-N, N] \times M_{0}$ with corners smoothed out - for example, we may take a compact simple manifold with boundary $M_{0}^{\prime} \subset M_{0}^{\circ}$, such that the interior of $[-N, N] \times M_{0}^{\prime}$ contains $M$, and take $M^{\prime}$ to be a smoothed out version of this. Hence $M^{\prime}$ is homeomorphic to a ball, and the exterior of $M^{\prime}$ in $[-N, N] \times M_{0}$ is homeomorphic to an $n$-dimensional annulus. Now we can make the following reduction:

Proposition 6.8 (Reduction to the convex case). Let $U$ and $V$ be two sections of $C^{\infty}\left(M^{\prime}\right.$; $\mathbb{C}^{m \times m}$ ) which solve solve $\mathcal{L}_{A_{1}, Q_{1}} U=0$ and $\mathcal{L}_{A_{2}, Q_{2}^{*}} V=0$. Then we have

$$
\int_{M^{\prime}}\left\langle\left(Q_{1}-Q_{2}+\left|A_{2}\right|^{2}-\left|A_{1}\right|^{2}\right) U, V\right\rangle+\int_{M^{\prime}}\left\langle\left(U\left(d V^{*}\right)-(d U) V^{*}, A_{2}-A_{1}\right)\right\rangle=0
$$

In particular, if $Q_{1}=Q_{2}=0$, then we also have that $\Lambda_{A_{1}}=\Lambda_{A_{2}}$ in $M^{\prime}$.

\footnotetext{
${ }^{2}$ Moreover, one can show that $\frac{\partial \Phi_{1}}{\partial \bar{z}}=-\frac{1}{2} \operatorname{tr}\left(\left(A_{1}\right)_{1}+i\left(A_{1}\right)_{r}\right)$ and $\frac{\partial \bar{\Phi}_{2}}{\partial \bar{z}}=\frac{1}{2} \operatorname{tr}\left(\left(A_{2}\right)_{1}+i\left(A_{2}\right)_{r}\right)$, but we will not need this here.

${ }^{3}$ The Plemelj-Sokhotski-Privalov formula actually gives $F^{-1}(z)=\frac{1}{2 \pi i} \int_{\partial M_{0}^{\eta}} \frac{C_{2}^{*}(\zeta) C_{1}(\zeta)}{\zeta-z} d \zeta$.
} 
Proof. Recall that we extended $A_{1}$ and $A_{2}$ to the whole of $\mathbb{R} \times M_{0}$ such that $A_{1}=A_{2}$ outside $M$; similarly, we extend $Q_{1}$ and $Q_{2}$ to have compact support and such that $Q_{1}=Q_{2}$ outside $M$ (allowed by boundary determination).

Then the proof follows immediately after applying Theorem 2.7 to the restrictions $\left.U\right|_{M}$ and $\left.V\right|_{M}$, which solve the appropriate equations in $M$, and the fact that $A_{1}=A_{2}$ and $Q_{1}=Q_{2}$ outside $M$. The final conclusion follows since $U$ and $V$ were arbitrary.

Let us denote by $L$ the connected component of $[-N, N] \times \partial M_{0}$ in $\mathbb{R} \times M_{0} \backslash M^{\circ}$. Furthermore, in this setting, we have the following:

Lemma 6.9. We have $G\left(x_{1}, x, v\right)$ equal to the identity for $\left(x_{1}, x\right) \in L$ and $v \in S_{x} M_{0}$.

In particular, $G$ is equal to identity on the complement of $M^{\prime}$ in $\mathbb{R} \times M_{0}$.

Proof. Let us fix a point $p \in \partial D$ and the polar coordinate $\eta \in S^{n-2}$ with $(p, \eta) \in \partial_{+} S D$. We have that $\tilde{A}=0$ outside $M^{\eta}$ and it would suffice to show $G=I d$ on the connected component of $\partial M_{0}^{\eta}$ in $M_{0}^{\eta} \backslash M^{\eta}$, that we denote by $L^{\eta}$. In $M_{0}^{\eta} \backslash M^{\eta}$, the equation 6.12 becomes:

$$
2 \frac{\partial G}{\partial \bar{z}}=\left[G,\left(A_{1}\right)_{1}+i\left(A_{1}\right)_{r}\right]
$$

and thus we also have $2 \frac{\partial G^{\prime \prime}}{\partial \bar{z}}=\left[G^{\prime \prime},\left(A_{1}\right)_{1}+i\left(A_{1}\right)_{r}\right]$, where $G^{\prime \prime}=G-I d$, with $\left.G^{\prime \prime}\right|_{\partial M_{0}^{\eta}}=0$. Since $\frac{\partial}{\partial \bar{z}}$ is an elliptic operator and the previous equation is a linear one, we may apply the unique continuity theorem for linear elliptic first order systems (see 7 for a precise statement) and conclude that $G^{\prime \prime}\left(p, \eta, x_{1}, r\right)=0$ for $z \in L^{\eta}$, since $G^{\prime \prime}=0$ on a codimension one set, thus proving the claim.

More precisely, note that $\left.G^{\prime \prime}\right|_{\partial M_{0}^{\eta}}=0$ implies that $\left.d G^{\prime \prime}\right|_{\partial M_{0}^{\eta}}=0$ and so we may extend $G^{\prime \prime}$ by zero slightly outside $M_{0}^{\eta}$ to a $C^{\infty}$ function by elliptic regularity. Then by the mentioned $\mathrm{UCP}$ we get $G^{\prime \prime}=0$ on $N^{\eta}$.

In particular, we also have $G\left(x_{1}, x, v\right)=I d$ for $\left(x_{1}, x\right)$ in the connected component of $\partial M$ in $L$ (this is non-empty and open in $\partial M$ ) and $v \in S_{x} M_{0}$, by the previous lemma. Call this component $\Gamma$.

Often, the crux of the matter in the X-ray injectivity problems is to prove the independence of the gauge of the velocity variable; the only difference here from the usual problem is that we have a complex derivative $\mathbb{X}$, instead of the usual geodesic vector field $X$. Indeed, we have:

LEmma 6.10. If the solution of 6.18 is independent of the velocity variable, then $G$ is a gauge equivalence between $A_{1}$ and $A_{2}$ on $E$, with $\left.G\right|_{\Gamma}=I d$.

Proof. It is easy to show the following fact about the geodesic vector field: $X(x, v) f=$ $d f(v)$, when $f$ is independent of the velocity variable. Therefore, we can write down two equations out of (6.18), one for $v$ and the other for $-v$, respectively:

$$
\begin{aligned}
& \frac{\partial G}{\partial x_{1}}+i d G(v)=-A_{1}\left(\frac{\partial}{\partial x_{1}}\right) G-i A_{1}(v) G+G A_{2}\left(\frac{\partial}{\partial x_{1}}\right)+i G A_{2}(v) \\
& \frac{\partial G}{\partial x_{1}}-i d G(v)=-A_{1}\left(\frac{\partial}{\partial x_{1}}\right) G+i A_{1}(v) G+G A_{2}\left(\frac{\partial}{\partial x_{1}}\right)-i G A_{2}(v)
\end{aligned}
$$

by adding and subtracting the above equations, we easily get that $d G=-A_{1} G+G A_{2}$ or equivalently $G^{*}\left(A_{1}\right)=G^{-1} d G+G^{-1} A_{1} G=A_{2}$, which together with $\left.G\right|_{\Gamma}=I d$ finishes the proof.

Ideally we would like to reduce this to an ordinary X-ray injectivity problem on $M_{0}$ (technically, 6.18 would become an injectivity problem for $G-I d$, with the inhomogeneous term 
equal to $\left.\tilde{A}^{1}+i \tilde{A}^{r}\right)$ in some process of excluding the $x_{1}$ variable. This is indeed possible for the line bundle case (similar to what we will see in the next chapter) - it involves the procedure of taking the logarithm of $G$ and applying the Fourier transform. Moreover, let us emphasise that all the information we obtained from the DN map through CGO solutions, we managed to pack into a single boundary condition: $G\left(x_{1}, x, v\right)=I d$ for $\left(x_{1}, x\right) \in \Gamma$ and $v \in S_{x} M_{0}$. Finally, for complete clarity, let us state the main problem separately:

Question 6.11 (The non-abelian $X$-ray transform or Radon transform). Let $\left(M_{0}, g_{0}\right)$ be a compact simple manifold with boundary, with $\operatorname{dim} M_{0} \geq 2$ and let $M$ be an isometrically embedded, compact submanifold of $T=\left(\mathbb{R} \times M_{0}, e \oplus g_{0}\right)$ with non-empty boundary and $\operatorname{dim} M=$ $\operatorname{dim} T$. Let $E=\mathbb{R} \times M_{0} \times \mathbb{C}^{m}$ be a Hermitian vector bundle equipped with two unitary connections $A_{1}$ and $A_{2}$, which are compactly supported and satisfy $A_{1}=A_{2}$ on $\mathbb{R} \times M_{0} \backslash M$. Let $R^{\prime}=$ $\left\{\left(x_{1}, x, v\right) \in \mathbb{R} \times S M_{0}:\left(x_{1}, x\right) \notin M\right\}$. Assume we are given a smooth matrix function $G$ : $\mathbb{R} \times S M_{0} \rightarrow G L(m, \mathbb{C})$ such that, if $\mathbb{X}=\frac{\partial}{\partial x_{1}}+i X$, where $X$ is the geodesic vector field:

$$
\mathbb{X} G\left(x_{1}, x, v\right)=-A_{1}\left(x_{1}, x\right)\left(\frac{\partial}{\partial x_{1}}+i v\right) G\left(x_{1}, x, v\right)+G\left(x_{1}, x, v\right) A_{2}\left(x_{1}, x\right)\left(\frac{\partial}{\partial x_{1}}+i v\right)
$$

for all $\left(x_{1}, x, v\right)$, with the additional condition $\left.G\right|_{R^{\prime}}=I d$. Prove that $G$ is independent of the velocity variable $v$.

It turns out that, under additional assumptions, we have $G$ equal to the identity on the whole of $\partial M$ :

Proposition 6.12. If the answer to Question 6.11 is positive, and $G^{*}\left(Q_{1}\right)=Q_{2}$, then $\left.G\right|_{\partial M}=I d$.

Proof. By Lemma 6.9, we have that $G$ is equal to the identity on the outside of $M^{\prime}$; thus, by the hypothesis and Lemma 6.10 we have $G^{*}\left(A_{1}\right)=A_{2}$. Moreover, we have $\left.G\right|_{\Gamma}=I d$ and we want to prove that $\left.G\right|_{\partial M}=I d$.

Let $F \in C^{\infty}\left(\partial M ; \mathbb{C}^{m \times m}\right)$ and assume smooth $U$ and $V$ solve $\mathcal{L}_{A_{1}, Q_{1}} U=0$ and $\mathcal{L}_{A_{2}, Q_{2}} V=0$ with the boundary condition $\left.U\right|_{\partial M}=\left.V\right|_{\partial M}=F$. By the DN map equality and the assumption on the gauges of $A_{1}$ and $A_{2}$ (normal components equal to zero near $\partial M$ ), we have $\left.\partial_{\nu} U\right|_{\partial M}=$ $\left.\partial_{\nu} V\right|_{\partial M}$. The hypothesis on $G$ implies that $U^{\prime}:=G V$ satisfies $\mathcal{L}_{A_{1}, Q_{1}} U^{\prime}=0$ and $\left.U^{\prime}\right|_{\Gamma}=\left.F\right|_{\Gamma}$. Moreover, we have on $\Gamma$ :

$$
\partial_{\nu}\left(U^{\prime}\right)=\iota_{\nu}((d G) V+G(d V))=\iota_{\nu}\left(G A_{2} V-A_{1} G V+G(d V)\right)=\partial_{\nu}(V)=\partial_{\nu}(U)
$$

So by the UCP for elliptic systems (see Remark 7.8), we have $U \equiv U^{\prime}$ and so $\left.G\right|_{\partial M} \equiv I d$, as $F$ was arbitrary.

Remark 6.13. Notice that if $Q_{1}=Q_{2}=0$, Proposition 6.8 implies that $\Lambda_{A_{1}}=\Lambda_{A_{2}}$ on $M^{\prime}$, so the problem is reduced to proving uniqueness (up to gauges) on $M^{\prime}$. More precisely, a gauge $G$ between $A_{1}$ and $A_{2}$ on $M^{\prime}$, equal to the identity on $\partial M^{\prime}$, would by uniqueness of first order equations and $G^{*}\left(A_{1}\right)=A_{2}$ imply $G=I d$ on $\Gamma$, so we may apply Proposition 6.12 to get $G=I d$ on $\partial M$.

REMARK 6.14. There is a way of formulating Question 6.11 in a more compact way. Namely, one could define the unitary connection $\hat{A}(R)=A_{1} R-R A_{2}$ on the endomorphism bundle of $E$ to get the form of the equation to $\mathbb{X} G+\hat{A}\left(\frac{\partial}{\partial x_{1}}+i v\right) G=0$. Then we may formulate the problem in terms of just a single connection.

\footnotetext{
${ }^{4}$ In particular, note that the condition on potentials holds if $Q_{1}=Q_{2}=0$.
} 
REMARK 6.15. If $A_{1}$ and $A_{2}$ are independent of the $x_{1}$ variable (on $M$ ) in the setting of Question 6.11. then we would have $A_{1} \equiv A_{2}$ by the boundary condition and therefore $G \equiv I d$.

Therefore, the problem is reduced to a new kind of a non-abelian X-ray transform. We leave it as one of the future projects to either further reduce the problem to an attenuated X-ray transform on $M_{0}$ or apply some other method to prove independence of the velocity variable directly. However, one thing is expected: methods from complex analysis and geometry could be useful to prove Question 6.11. This is supported by the work of Eskin (see Section 5 in [26]), where he proves Conjecture $\mathrm{A}$ in the Euclidean metric case, by "moving around" the $x_{1}$ direction, which can be interpreted as having the equations 6.12 for essentially all planes going through points in $M$. In short, by generating a holomorphic family of such planes, Eskin obtains that $G$ is holomorphic with respect to this variable and hence constant by Liouville's theorem; such families are dense enough to guarantee $G$ is constant in the vertical directions and hence independent of $v$. Unlike the Euclidean metric case, in our situation we have a fixed $x_{1}$ direction, so we may also expect a different approach to be used. 


\section{CHAPTER 7}

\section{Proof of the Main Theorem I}

In this chapter, we prove prove our main application of the construction of Gaussian Beams in Chapter 5 - Theorem E. By taking the CGO solutions into the integral identity and reducing the problem to an X-ray transform, we are able to prove that $d A_{1}=d A_{2}$ if $\Lambda_{A_{1}}=\Lambda_{A_{2}}$. For the case of partial data, one should take extra care to deal with the leftover terms. The chapter is concluded with a discussion of how holonomy determines the gauge class of the connection and proving that $d A_{1}=d A_{2}$ is actually enough to gauge identify two connections, by using the unique continuation principle.

\subsection{The main recovery}

We assume that we are in the CTA setting with $(M, g) \Subset\left(\mathbb{R} \times M_{0}\right)$ and we write $\tilde{g}=e \oplus g_{0}$ for the product metric; we ask that $g=c \tilde{g}$ for some positive function $c$. We start with the full data case and then move on to partial data.

Theorem 7.1 (Main recovery for full data). Suppose $A_{1}$ and $A_{2}$ are two unitary connections on $E=M \times \mathbb{C}$ and that the DN maps $\Lambda_{A_{1}}=\Lambda_{A_{2}}$ are the same. If the geodesic ray transform on $M_{0}$ is injective on 1-forms and functions, then we must have $d A_{1}=d A_{2}$.

Proof. Let $\tilde{A}=A_{2}-A_{1}$. By Theorem 4.10 , we have the solutions

$$
u=e^{-(\tau+i \lambda) x_{1}} c^{-\frac{n-2}{4}}\left(v_{1}+r_{1}\right) \quad \text { and } \quad v=e^{(\tau+i \lambda) x_{1}} c^{-\frac{n-2}{4}}\left(v_{2}+r_{2}\right)
$$

to the equations $\mathcal{L}_{A_{1}} u=0$ and $\mathcal{L}_{A_{2}} v=0$, with the desired concentration and decay properties. It is worth noting that $v_{i}$ s are defined on the whole $J_{0} \times M_{0}$, where $J_{0}=[-N, N]$ for some large $N$ and $r_{i}$ s on $M$. By applying Theorem 2.6, we obtain the following equality ( $d V_{g}$ is the volume form):

$$
\int_{M}\left(\left|A_{2}\right|_{g}^{2}-\left|A_{1}\right|_{g}^{2}\right) u \bar{v} d V_{g}+\int_{M}\left\langle u d \bar{v}-\bar{v} d u, A_{2}-A_{1}\right\rangle_{g} d V_{g}=0
$$

Observe that in the first factor we have $q_{i}$ s and $A_{i}$ s bounded, which together with $L^{2}$ bounds on $v_{i}$ s and $r_{i}$ s from the construction theorem gives us that the first term is equal to $O(1)$. Now, we will divide by $\tau$ and take the $\tau \rightarrow \infty$ limit. First note that:

$$
\begin{aligned}
\bar{v} d u=e^{-2 i \lambda x_{1}} c^{-\frac{n-2}{2}}\left(\left(c^{\frac{n-2}{4}} d\left(c^{-\frac{n-2}{4}}\right)-(\tau+i \lambda)\right) d x_{1}\left(\bar{v}_{2}+\bar{r}_{2}\right)\left(v_{1}+r_{1}\right)\right. & \\
+ & \left.+\left(\bar{v}_{2}+\bar{r}_{2}\right)\left(d v_{1}+d r_{1}\right)\right)
\end{aligned}
$$

and a similar formula holds for $u d \bar{v}$. The factor containing the derivative of $c$ will be zero in the limit, when divided by $\tau$. Therefore, when plugging in these expressions in $(7.2)$, we can neglect the $r_{i}$ factors and hence obtain the limit:

$$
\begin{aligned}
\lim _{\tau \rightarrow \infty} \frac{1}{\tau} \int_{M}\langle\tilde{A}, \bar{v} d u\rangle_{g} d V_{g} & =\lim _{\tau \rightarrow \infty} \frac{1}{\tau} \int_{M} e^{-2 i \lambda x_{1}} c^{-\frac{n-2}{2}} \frac{1}{c}\left\langle\tilde{A},(-\tau+i \lambda) v_{1} \bar{v}_{2} d x_{1}\right. \\
& \left.+\bar{v}_{2} d v_{1}\right\rangle_{\tilde{g}} c^{\frac{n}{2}} d V_{\tilde{g}}=\int_{0}^{L} \int_{-\infty}^{\infty} e^{-2 i \lambda x_{1}}\left(-\tilde{A}_{1}+i \tilde{A}_{t}\right) e^{\Phi_{1}+\bar{\Phi}_{2}} e^{-2 \lambda t} d x_{1} d t
\end{aligned}
$$


where, in the second line we have gone from the integral over $M$ to an integral over $\mathbb{R} \times M_{0}$; this is allowed since, by the boundary determination result Theorem 3.4. we can assume that $\left.\tilde{A}\right|_{\partial M}=0$ to infinite order. Moreover, we may pick $N$ such that the interior of $J_{0} \times M_{0}$ contains the supports of extensions of $A_{1}$ and $A_{2}$.

Also, we used that the inner product on forms is given by the inverse of the metric $g$; hence the $\frac{1}{c}$ factor cancels with the other $c$ factors. The $\Phi_{i}$ functions satisfy the equations 4.1, where $X=-2 g^{i j}\left(A_{1}\right)_{i} \frac{\partial}{\partial x^{j}}$ and $Y=-2 g^{i j}\left(A_{2}\right)_{i} \frac{\partial}{\partial x^{j}}$ are the first order terms of the connection Laplacian:

$$
\frac{\partial \Phi_{1}}{\partial z}=\frac{1}{2}\left(-\left(A_{1}\right)_{1}+i\left(A_{1}\right)_{t}\right) \quad \text { and } \quad \frac{\partial \bar{\Phi}_{2}}{\partial z}=\frac{1}{2}\left(\left(A_{2}\right)_{1}-i\left(A_{2}\right)_{t}\right)
$$

where $z=x_{1}+i t$ is the complex variable and $\bar{z}=x_{1}-i t$ is its conjugate. By summing the two equations, we get:

$$
\frac{\partial\left(\Phi_{1}+\bar{\Phi}_{2}\right)}{\partial z}=\frac{1}{2}\left(\tilde{A}_{1}-i \tilde{A}_{t}\right)
$$

Now we obtain a similar expression for the $u d \bar{v}$ part, namely:

$$
\lim _{\tau \rightarrow \infty} \frac{1}{\tau} \int_{M}\langle\tilde{A}, u d \bar{v}\rangle=\int_{0}^{L} \int_{-\infty}^{\infty} e^{-2 i \lambda x_{1}}\left(\tilde{A}_{1}-i \tilde{A}_{t}\right) e^{\Phi_{1}+\bar{\Phi}_{2}} e^{-2 \lambda t} d x_{1} d t
$$

and finally obtain the limit for $(7.2)$ :

$$
0=\int_{0}^{L} \int_{-\infty}^{\infty} e^{-2 i \lambda x_{1}}\left(\tilde{A}_{1}-i \tilde{A}_{t}\right) e^{\Phi_{1}+\bar{\Phi}_{2}} e^{-2 \lambda t} d x_{1} d t
$$

By using Stokes' theorem and noting that $d z \wedge d \bar{z}=2 i d x_{1} \wedge d t$, together with (7.5), on a smooth subdomain $\Omega \subset \mathbb{R} \times[0, L]$ which contains the support of $\tilde{A}$ :

$$
0=\int_{\Omega} d\left(e^{-2 i \lambda x_{1}} e^{-2 \lambda t} e^{\Phi_{1}+\bar{\Phi}_{2}} d \bar{z}\right)=\int_{\partial \Omega} e^{-2 i \lambda x_{1}} e^{-2 \lambda t} e^{\Phi_{1}+\bar{\Phi}_{2}} d \bar{z}
$$

Now by exploiting the fact that we could put an arbitrary anti-holomorphic $h$ as a multiplier of $e^{\Phi_{1}}$, we obtain the integral identity:

$$
0=\int_{\partial \Omega} e^{-2 i \lambda x_{1}} e^{-2 \lambda t} h e^{\Phi_{1}+\bar{\Phi}_{2}} d \bar{z}
$$

for all such $h$. Let us take $\Omega$ simply-connected, e.g. $\Omega=J_{0} \times[0, L]$ (smoothed out at the corners). This means that upon conjugating, by Lemma 6.7, the restriction of the function $e^{\bar{\Phi}_{1}+\Phi_{2}}$ at the boundary is a restriction of a non-vanishing holomorphic function $F$, defined on $\Omega$, i.e. $\left.F\right|_{\partial \Omega}=\left.e^{\bar{\Phi}_{1}+\Phi_{2}}\right|_{\partial \Omega}$. Moreover, since $\Omega$ is simply-connected, we can find a logarithm, so that $F=e^{G}$, where $G$ is holomorphic and we may assume $\left.G\right|_{\partial \Omega}=\bar{\Phi}_{1}+\Phi_{2}$. After using Stokes' theorem again with $\bar{h}=G e^{-G}$, we obtain:

$$
0=\int_{\Omega} e^{2 \lambda i\left(x_{1}+i t\right)}\left(\tilde{A}_{1}+i \tilde{A}_{t}\right) d z \wedge d \bar{z}
$$

and so finally:

$$
0=\int_{0}^{L} \int_{-\infty}^{\infty} e^{2 \lambda i\left(x_{1}+i t\right)}\left(\tilde{A}_{1}+i \tilde{A}_{t}\right) d x_{1} d t
$$

Let us define:

$$
\begin{aligned}
& f\left(\lambda, x^{\prime}\right)=\int_{-\infty}^{\infty} e^{i \lambda x_{1}} \tilde{A}_{1}\left(x_{1}, x^{\prime}\right) d x_{1}=\mathcal{F}\left(\tilde{A}_{1}\right)\left(\lambda, x^{\prime}\right) \\
& \alpha\left(\lambda, x^{\prime}\right)=\sum_{j=2}^{n}\left(\int_{-\infty}^{\infty} e^{i \lambda x_{1}} \tilde{A}_{j}\left(x_{1}, x^{\prime}\right) d x_{1}\right) d x^{j}=\sum_{j=2}^{n} \mathcal{F}\left(\tilde{A}_{j}\right)\left(\lambda, x^{\prime}\right) d x^{j}
\end{aligned}
$$


where $\mathcal{F}$ denotes the Fourier transform; we will write $\mathcal{F}(\alpha)$ for the Fourier transform of a compactly supported 1-form $\alpha$ on $\mathbb{R} \times M_{0}$. With this notation, the identity above becomes (replace $2 \lambda$ with $\lambda$ without loss of generality and relabel $t$ by $r$ ):

$$
0=\int_{0}^{L} e^{-\lambda r}(f+i \alpha(\dot{\gamma}(r))) d r
$$

along any unit speed, non-tangential geodesic in $M_{0}$. We would like to use the fact that the geodesic transform is injective as much as we can, even though we obtained an attenuated transform. Thus we set $\lambda=0$ and use the injectivity of the ray transforms to get $\alpha\left(0, x^{\prime}\right)=-i d p_{0}$ and $f\left(0, x^{\prime}\right)=0$ for some smooth $p_{0}$ such that $\left.p_{0}\right|_{\partial M_{0}}=0$. Furthermore, we can take the $\frac{\partial}{\partial \lambda}$ derivative of the integral to get:

$$
\int_{0}^{L} e^{-\lambda r}\left(-r(f+i \alpha)+\frac{\partial}{\partial \lambda}(f+i \alpha)\right) d r=0
$$

Again we plug in $\lambda=0$ and use injectivity, together with the following calculation:

$$
\int_{0}^{L} r i \alpha d r=\int_{0}^{L} r \frac{\partial p_{0}}{\partial r} d r=-\int_{0}^{L} p_{0} d r
$$

where we used the fact that $p_{0}$ vanishes at the boundary. Now using that $f=0$, we obtain at $\left(0, x^{\prime}\right)$ for all $x^{\prime} \in M_{0}$ :

$$
p_{0}+\frac{\partial f}{\partial \lambda}=0 \quad \text { and } \quad \frac{\partial \alpha}{\partial \lambda}=-i d p_{1}
$$

for some smooth $p_{1}$ which vanishes at the boundary. It is now clear how we are going to proceed with this inductively, but let us go one step further for clarity. Taking another derivative with respect to $\lambda$, we have:

$$
\int_{0}^{L} e^{-\lambda r}\left(r^{2}(f+i \alpha)-2 r \frac{\partial(f+i \alpha)}{\partial \lambda}+\frac{\partial^{2}(f+i \alpha)}{\partial \lambda^{2}}\right)=0
$$

Now by partial integration and using the properties of $p_{0}, p_{1}$, we have:

$$
\int_{0}^{L} r^{2} i \alpha d r=-\int_{0}^{L} 2 r p_{0} d r \quad \text { and } \quad \int_{0}^{L} r i \frac{\partial \alpha}{\partial \lambda}=-\int_{0}^{L} p_{1} d r
$$

Therefore, by plugging in $\lambda=0$ and substituting:

$$
\int_{0}^{L}\left(\left(2 p_{1}+\frac{\partial^{2} f}{\partial \lambda^{2}}\right)+i \frac{\partial^{2} \alpha}{\partial \lambda^{2}}\right) d r=0
$$

Again, we get some smooth $p_{2}$ vanishing at the boundary such that $\frac{\partial^{2} \alpha}{\partial \lambda^{2}}=-i d p_{2}$ and $2 p_{1}+\frac{\partial^{2} f}{\partial \lambda^{2}}=$ 0 .

Now, let us assume inductively that $\frac{\partial^{j} \alpha}{\partial \lambda^{j}}=-i d p_{j}$ and $j p_{j-1}+\frac{\partial^{j} f}{\partial \lambda^{j}}=0$, for $j=0,1, \ldots, n-1$ and $p_{j}$ are smooth functions on $M_{0}$ vanishing at the boundary (with $p_{-1}=0$ predefined). We will prove the existence of $p_{n}$ by induction. Let us define:

$$
S=\frac{\partial^{n}}{\partial \lambda^{n}} \int_{0}^{L} e^{-\lambda r}(f+i \alpha) d r=\int_{0}^{L} e^{-\lambda r} \sum_{j=0}^{n}\left(\left(\begin{array}{c}
n \\
j
\end{array}\right)(-1)^{j} r^{j} \frac{\partial^{n-j}(f+i \alpha)}{\partial \lambda^{n-j}}\right) d r=0
$$

Now, using the following formulas for $\lambda=0$ :

$$
\int_{0}^{L} r^{i} \frac{\partial^{n-i} f}{\partial \lambda^{n-i}} d r=\int_{0}^{L} r^{i}\left(-(n-i) p_{n-i-1}\right) d r=-(n-i) \int_{0}^{L} r^{i} p_{n-i-1} d r
$$

valid for $i>0$ and:

$$
\int_{0}^{L} r^{k} \frac{\partial^{n-k}(i \alpha)}{\partial \lambda^{n-k}} d r=\int_{0}^{L} r^{k} \frac{\partial p_{n-k}}{\partial r} d r=-k \int_{0}^{L} r^{k-1} p_{n-k} d r
$$


for $k>0$, and inserting them in the expression for $S$, we get:

$$
\begin{aligned}
S= & \int_{0}^{L} \frac{\partial^{n}(f+i \alpha)}{\partial \lambda^{n}} d r+\sum_{j=1}^{n}\left(\begin{array}{c}
n \\
j
\end{array}\right)(-1)^{j} \int_{0}^{L}\left(\left(-(n-j) r^{j} p_{n-j-1}-j r^{j-1} p_{n-j}\right)\right) d r \\
& =\int_{0}^{L} r^{0}\left(\frac{\partial^{n}(f+i \alpha)}{\partial \lambda^{n}}+n p_{n-1}\right) d r+\int_{0}^{L} r^{1}\left(n(n-1) p_{n-2}-2 p_{n-2} \cdot \frac{n(n-1)}{2}\right) d r+ \\
& +\cdots+\int_{0}^{L} r^{j}\left(\left(\begin{array}{c}
n \\
j
\end{array}\right)(-1)^{j+1}(n-j) p_{n-j-1}-(j+1) p_{n-j-1}\left(\begin{array}{c}
n \\
j+1
\end{array}\right)(-1)^{j+1}\right) d r+\cdots \\
& =\int_{0}^{L}\left(\frac{\partial^{n}(f+i \alpha)}{\partial \lambda^{n}}+n p_{n-1}\right) d r=0
\end{aligned}
$$

where the last line is true by cancelling the expressions in the brackets for $r^{j}$, where $j>0$. Therefore, by the injectivity of the X-ray transform we have $\frac{\partial^{n} f}{\partial \lambda^{n}}+n p_{n-1}=0$ and $\frac{\partial^{n}(\alpha)}{\partial \lambda^{n}}=-i d p_{n}$, for some smooth $p_{n}$ vanishing at the boundary. This finishes the proof by induction.

From $(7.9)$ it follows that $\left.\frac{\partial^{k} f}{\partial \lambda^{k}}\right|_{\lambda=0} \leq C^{k}$ for some positive $C$ and all $k$, so we see that

$$
\beta\left(\lambda, x^{\prime}\right):=-\sum_{k=0}^{\infty} p_{k}\left(x^{\prime}\right) \frac{\lambda^{k}}{k !}
$$

converges and since the Fourier transform of a compactly supported function is analytic:

$$
f=\left.\sum_{k=0}^{\infty} \frac{\partial^{k} f}{\partial \lambda^{k}}\right|_{\lambda=0} \frac{\lambda^{k}}{k !}=-\sum_{k=0}^{\infty} k p_{k-1} \frac{\lambda^{k}}{k !}=\lambda \beta
$$

and similarly, by using the relation $\frac{\partial^{j} \alpha}{\partial \lambda^{j}}=-i d p_{j}$ (for all $j \geq 0$ ) we get that

$$
\alpha=\left.\sum_{k=0}^{\infty} \frac{\partial^{k} \alpha}{\partial \lambda^{k}}\right|_{\lambda=0} \frac{\lambda^{k}}{k !}=-i \sum_{k=0}^{\infty} d p_{k} \frac{\lambda^{k}}{k !}=i d^{\prime} \beta
$$

where $d^{\prime}$ denotes exterior differentiation in $M_{0}$. Coming back to the main proof, we see that:

$$
\sum_{2 \leq j<k} \mathcal{F}\left(\partial_{j} \tilde{A}_{k}-\partial_{k} \tilde{A}_{j}\right) d x^{j} \wedge d x^{k}=d^{\prime} \alpha=0
$$

Again, the Fourier transforms of the quantities on the left hand side are analytic and thus $\partial_{j} \tilde{A}_{k} \equiv \partial_{k} \tilde{A}_{j}$ for all $j, k \geq 2$. Furthermore, since we have

$$
\mathcal{F}\left(\partial_{j} \tilde{A}_{1}-\partial_{1} \tilde{A}_{j}\right)=\partial_{j} f+i \lambda \alpha_{j}=0
$$

for all $j \geq 2$ by 7.11 and 7.12 , in the same manner as before we have that $\partial_{j} \tilde{A}_{1} \equiv \partial_{1} \tilde{A}_{j}$; gluing this information together, we finally conclude that $d \tilde{A}=0$ or equivalently that $d A_{1}=d A_{2}$. This finishes the proof.

Now we depart to partial data, which is more technical. More precisely, we have to worry about the leftover terms in the partial integration and how we extend the connections outside $M$, since now boundary determination works only on a part of the boundary, so $A_{1}-A_{2}$ is only $L^{\infty}$ when extended by zero.

Theorem 7.2 (Partial boundary data case). In the same notation as in Theorem 7.1, we prove $d A_{1}=d A_{2}$ given $\left.\Lambda_{A_{1}}\right|_{\Gamma}=\left.\Lambda_{A_{2}}\right|_{\Gamma}$, where $\Gamma$ is a neighbourhood of the front side $\partial M_{-}$.

Proof. We are still able to prove $d A_{1}=d A_{2}$ as follows. We think of the point $x_{0}$ in Theorem 1.1 from $\mathbf{2 2}$ as the point at "infinity" so that the rays are straight lines along the $x_{1}$ axis. 
Let us use the notation

$$
F_{\epsilon}=\left\{x \in \partial M \mid\left\langle\frac{\partial}{\partial x_{1}}, \nu(x)\right\rangle=\nu_{1}(x)<\epsilon\right\}
$$

for any positive $\epsilon>0$; we also denote $B_{\epsilon}=\partial M \backslash F_{\epsilon}$. We pick $\epsilon$ small enough such that $F_{\epsilon} \subset \Gamma$. Consider the CGO solutions $u$ and $v$ to $\mathcal{L}_{A_{1}} u=\mathcal{L}_{A_{2}} v=0$ such that $\left.u\right|_{\partial M}=f$ and $\left.v\right|_{\partial M}=g$, of the form in (7.1). Then the assumption on the DN map gives us a smooth $w$, such that $\mathcal{L}_{A_{1}} w=0,\left.w\right|_{\partial M}=g$ and $\left.\partial_{\nu} w\right|_{\Gamma}=\left.\partial_{\nu} v\right|_{\Gamma}$. Theorem 2.6 gives us (we plug in $A_{1}$ for $B$ and $A_{2}$ for $A$, so some terms swap places):

$$
\int_{\partial M \backslash F_{\epsilon}}\left\langle\partial_{\nu}(v-w), f\right\rangle=\int_{M}\left(\left|A_{1}\right|^{2}-\left|A_{2}\right|^{2}\right) v \bar{u}+\int_{M}\left\langle v d \bar{u}-\bar{u} d v, A_{1}-A_{2}\right\rangle
$$

Observe (recall) we have the following relations: $F(-\infty)=F=\partial M_{-}, B_{\epsilon} \subset \partial M_{+}$and also $\left\langle\frac{\partial}{\partial x_{1}}, \nu\right\rangle=\nu_{1}(x) \geq \epsilon$ on $B_{\epsilon}$.

We claim that the term on the left hand side of $(7.13)$ is equal to $O\left(|\tau|^{\frac{1}{2}}\right)$ as $|\tau| \rightarrow \infty-$ it is bounded by (using Cauchy-Schwarz)

$$
\frac{1}{\sqrt{\epsilon}}\left\|\sqrt{\partial_{\nu} x_{1}} e^{-\tau x_{1}} \partial_{\nu}(v-w)\right\|_{L^{2}\left(B_{\epsilon}\right)} \times\left\|c^{-\frac{n-2}{4}}\left(v_{1}+r_{1}\right)\right\|_{L^{2}\left(B_{\epsilon}\right)}
$$

which is in turn bounded (up to constant) by the following expression, by applying the Carleman estimate with the boundary part (5.7), since $\left.(v-w)\right|_{\partial M}=0$ :

$$
\left.\begin{array}{rl}
\frac{1}{\sqrt{\epsilon}}\left(\sqrt{h}\left\|e^{-\tau x_{1}} \mathcal{L}_{A_{1}}(v-w)\right\|_{L^{2}(M)}+\left\|\sqrt{-\partial_{\nu} x_{1}} e^{-\tau x_{1}} \partial_{\nu}(v-w)\right\|_{L^{2}\left(\partial M_{-}\right)}\right) & \\
\times\left(\left\|v_{1}\right\|_{L^{2}\left(B_{\epsilon}\right)}+\left\|r_{1}\right\| L^{2}(\partial M)\right.
\end{array}\right)
$$

The second summand in the first line of (7.14) is zero by the assumption; the first one is bounded by considering the following formula:

$$
\begin{aligned}
\mathcal{L}_{A_{1}}(v-w)=\mathcal{L}_{A_{1}} v=\left(\mathcal{L}_{A_{1}}\right. & \left.-\mathcal{L}_{A_{2}}\right) v \\
& =-2\left(A_{1}-A_{2}, d v\right)+d^{*}\left(A_{1}-A_{2}\right) v-\left(\left|A_{1}\right|^{2}-\left|A_{2}\right|^{2}\right) v=O(|\tau|)
\end{aligned}
$$

as $\left\|e^{-\tau x_{1}} d v\right\|=O(|\tau|)$ - by Remark 4.6 we have $\left\|d v_{2}\right\|_{L^{2}(M)}=O(|\tau|)$ and by the construction in Theorem 4.10 we have $\left\|r_{s}\right\|_{H^{1}(M)}=o(|\tau|)$. Therefore, the first line is equal to $O\left(|\tau|^{\frac{1}{2}}\right)$. We are left to prove the second line of 7.14 is equal to $O(1)$.

Firstly, observe that by the trace inequality, Proposition A.4. we have $\left\|r_{s}\right\|_{L^{2}(\partial M)} \lesssim\left\|r_{s}\right\|_{H^{1}(M)}$; note that in the previous paragraph we had $\left\|r_{s}\right\|_{H^{1}(M)}=o(|\tau|)$ - however, we can do better than that. By recalling Remark 4.11 (with $K=0$ ), we may assume that the $H^{1}$ norm of $r_{s}$ is bounded uniformly as $\tau \rightarrow \infty$ and hence, so is $\left\|r_{s}\right\|_{L^{2}(\partial M)}$.

Secondly, we want to prove that $\left\|v_{1}\right\|_{L^{2}\left(B_{\epsilon}\right)}=O(1)$ as $\tau \rightarrow \infty$ - this will be a bit more subtle, since we will crucially use the fact that we are taking the $L^{2}$ norm over $B_{\epsilon}$ (and not over $\left.\partial M_{+}\right)$. Without loss of generality, we assume that $\partial M_{\epsilon}=\partial M \cap \pi^{-1}(\epsilon)$ is a manifold, where $\pi: \partial M \rightarrow \mathbb{R}$ is the projection (follows from Sard's theorem). Thus $B_{\epsilon}$ is compact manifold with boundary, of dimension $(n-1)$.

Notice that the second projection $\pi_{2}: \partial M \rightarrow M_{0}$ is a local diffeomorphism on $B_{\eta}$ for any $\eta>0$. So if we pick an arbitrary point $p \in B_{\epsilon}$ and an open neighbourhood $U$ of $p$ such that $\left.\pi_{2}\right|_{U}$ a diffeomorphism, we see that $\pi_{2 *}\left(d V_{\partial M}\right)=J_{\pi_{2}} d V_{g_{0}}$ by the change of variables formula, where $J_{\pi_{2}}=\left|\operatorname{det} d \pi_{2}^{-1}\right|$ is the Jacobian. So by the properties of the integral we see that

$$
\int_{U \cap B_{\epsilon}}\left|v_{1}\right|^{2} d V_{\partial M}=\int_{\pi_{2}\left(U \cap B_{\epsilon}\right)}\left|v_{1} \circ \pi_{2}^{-1}\right|^{2} J_{\pi_{2}} d V_{g_{0}}
$$


Note that $\pi_{2}^{-1}(x)=\left(x_{1}(x), x\right)$ on $\pi_{2}(U)$, where $x_{1}(x)$ is a smooth function, which means that by taking small enough $U$ we have $J_{\pi_{2}}$ bounded locally. Therefore, by the estimate (4.6) in the construction of Gaussian Beams and the lines nearby, we locally have

$$
\int_{\pi_{2}\left(U \cap B_{\epsilon}\right)}\left|v_{1} \circ \pi_{2}^{-1}\right|^{2} J_{\pi_{2}} d V_{g_{0}}=O(1)
$$

as $\tau \rightarrow \infty$. Now since $B_{\epsilon}$ compact, we immediately obtain that $\left\|v_{1}\right\|_{L^{2}\left(B_{\epsilon}\right)}=O(1)$ as $\tau \rightarrow \infty$, which proves the claim.

Finally, if we quotient out by $\tau$ and take the limit $\tau \rightarrow \infty$ as before, we now have the left hand side going to zero by the estimate, which takes us back to the second step of the proof of Theorem 7.1 - what follows addresses the issue that $\tilde{A}$ does not have a smooth zero extension.

Firstly, consider smooth extensions $A_{1}^{\epsilon}$ and $A_{2}^{\epsilon}$ of $A_{1}$ and $A_{2}$ respectively, with supports in $M^{\epsilon}$, which we define as the manifold obtained by taking the union of $M$ and its exterior $\epsilon$-collar in $\mathbb{R} \times M_{0}$, for some small $\epsilon>0$. Let us also write $N^{\epsilon}=M^{\epsilon} \backslash M$ and $\tilde{A}^{\epsilon}=A_{2}^{\epsilon}-A_{1}^{\epsilon}$. We also denote the corresponding CGO solutions

$$
u^{\epsilon}=e^{-(\tau+i \lambda) x_{1}} c^{\frac{n-2}{4}}\left(v_{1}^{\epsilon}+r_{1}^{\epsilon}\right) \quad \text { and } \quad v^{\epsilon}=e^{(\tau+i \lambda) x_{1}} c^{\frac{n-2}{4}}\left(v_{2}^{\epsilon}+r_{2}^{\epsilon}\right)
$$

to $\mathcal{L}_{A_{1}^{\epsilon}} u^{\epsilon}=0$ and $\mathcal{L}_{A_{2}^{\epsilon}} v^{\epsilon}=0$ in $M^{\epsilon}$. Corresponding to these solutions, we have $\Phi_{1}^{\epsilon}$ and $\Phi_{2}^{\epsilon}$ that satisfy the following equations:

$$
\frac{\partial \Phi_{1}^{\epsilon}}{\partial z}=\frac{1}{2}\left(-\left(A_{1}^{\epsilon}\right)_{1}+i\left(A_{1}^{\epsilon}\right)_{t}\right)=: Z_{1}^{\epsilon} \quad \text { and } \quad \frac{\partial \overline{\Phi_{2}^{\epsilon}}}{\partial z}=\frac{1}{2}\left(\left(A_{2}^{\epsilon}\right)_{1}-i\left(A_{2}^{\epsilon}\right)_{t}\right)=: Z_{2}^{\epsilon}
$$

on $\mathbb{R} \times[0, L]$. More precisely, we have the following expressions given by the Cauchy operator:

$$
\Phi_{1}^{\epsilon}(\omega)=\frac{1}{2 \pi i} \int_{\mathbb{C}} \frac{Z_{1}^{\epsilon}(z)}{\bar{z}-\bar{\omega}} d z \wedge d \bar{z} \quad \text { and } \quad \Phi_{2}^{\epsilon}(\omega)=\frac{1}{2 \pi i} \int_{\mathbb{C}} \frac{\overline{Z_{2}^{\epsilon}}(z)}{z-\omega} d z \wedge d \bar{z}
$$

Moreover, we can still solve the equation (7.4), where we extend $A_{1}$ and $A_{2}$ by zero outside $M$ in the distributional sense (we denote them by the same letter) and obtain $\Phi_{1}, \Phi_{2} \in H_{l o c}^{1}(\mathbb{R} \times[0, L])$, satisfying the equations:

$$
\frac{\partial \Phi_{1}}{\partial z}=\frac{1}{2}\left(-\left(A_{1}\right)_{1}+i\left(A_{1}\right)_{t}\right)=: Z_{1} \quad \text { and } \quad \frac{\partial \overline{\Phi_{2}}}{\partial z}=\frac{1}{2}\left(\left(A_{2}\right)_{1}-i\left(A_{2}\right)_{t}\right)=: Z_{2}
$$

Furthermore, $\Phi_{1}$ and $\Phi_{2}$ have continuous representatives, which follows from the Dominated convergence theorem applied to the Cauchy integral formula in the polar coordinate system at $\omega \in \mathbb{R} \times[0, L]$, as follows (the analogous argument applies to $\Phi_{1}$ ):

$$
\Phi_{2}(\omega)=\frac{1}{2 \pi i} \int_{\mathbb{C}} \frac{\overline{Z_{2}}(z)}{z-\omega} d z \wedge d \bar{z}=\frac{1}{\pi} \int_{0}^{\infty} \int_{0}^{2 \pi} \overline{Z_{2}}\left(\omega+r e^{i \theta}\right) e^{-i \theta} d \theta d r
$$

So if $\omega_{k} \rightarrow \omega$, by the DCT we get that $\Phi_{2}\left(\omega_{k}\right) \rightarrow \Phi_{2}(\omega)$ and thus $\Phi_{2}$ is continuous.

Our next aim is to compute the limit in (7.3) as $\tau \rightarrow \infty$ and $\epsilon \rightarrow 0$ for the solutions $u^{\epsilon}$ and $v^{\epsilon}$ instead of $u$ and $v$, respectively and $\tilde{A}^{\epsilon}$ instead of $\tilde{A}$. This integral splits into an integral over $\mathbb{R} \times M_{0}$, the limit of which we know and a remainder integral over $N^{\epsilon}$ of the following type, that we would like to prove is small in the limit as $\epsilon \rightarrow 0$ :

$$
\lim _{\tau \rightarrow \infty} \frac{1}{\tau} \int_{N^{\epsilon}} e^{-2 i \lambda x_{1}}\left\langle\tilde{A}^{\epsilon},(-\tau+i \lambda) v_{1}^{\epsilon} \overline{v_{2}^{\epsilon}} d x_{1}+\overline{v_{2}^{\epsilon}} d v_{1}^{\epsilon}\right\rangle d V_{\tilde{g}}
$$

Firstly, observe that if $S \subset M_{0}$ is a compact submanifold with boundary and same dimension and $\gamma$ intersects the boundary of $S$ transversely, then

$$
\lim _{\tau \rightarrow \infty} \int_{\left\{x_{1}^{\prime}\right\} \times S} v_{1}^{\prime}{\overline{v^{\prime}}}_{2} \phi d V_{g_{0}}=\int_{\gamma^{-1}(S)} e^{\Psi_{1}+\bar{\Psi}_{2}} e^{-2 \lambda t} d t
$$


for $x_{1}^{\prime} \in J_{0}$, where $v_{1}^{\prime}$ and $v_{2}^{\prime}$ are some general Gaussian beams coming from our construction in Chapter 4, $\Psi_{1}$ and $\Psi_{2}$ are complex phases that satisfy the usual transport equations. Moreover, we have a similar formula involving the integrals of $\left\langle\alpha, d{\overline{v^{\prime}}}_{2}\right\rangle v_{1}^{\prime}$ and $\left\langle\alpha, d v_{1}^{\prime}\right\rangle{\overline{v^{\prime}}}_{2}$ for a one form $\alpha$ in the limit $\tau \rightarrow \infty$.

Secondly, recall that for almost all $x_{1} \in \mathbb{R}$ we have $\partial M \pitchfork\left\{x_{1}\right\} \times M_{0}$, by applying Sard's theorem to the projection $\pi$; denote the set of such $x_{1} \in J_{0}$ by $T$. This means that $\pi^{-1}\left(x_{1}\right) \cap \partial M$ is a manifold of dimension $n-2$ for almost all $x_{1}$ and moreover that $N_{x_{1}}^{\epsilon}:=\pi^{-1}\left(x_{1}\right) \cap N^{\epsilon}$ is a manifold of dimension $n-1$ with boundary for almost all $x_{1}$ (and similarly we set $M_{x_{1}}:=$ $\left.\pi^{-1}\left(x_{1}\right) \cap M\right)$.

Thirdly, we claim that for almost all geodesics $\gamma$ in $M_{0}$ and for almost all $x_{1} \in \mathbb{R}$, we have $\gamma \pitchfork \partial N_{x_{1}}^{\epsilon}$, where by $\gamma$ we mean the image of $\gamma$ and we identify subsets of $\{p\} \times M_{0}$ for some $p \in \mathbb{R}$ with subsets in $M_{0}$ as appropriate ( $\epsilon>0$ is fixed). To prove this, note first that the geodesics in $M_{0}$ are parametrised, by the influx boundary manifold $\Gamma:=\partial_{+} S M_{0}$ which has dimension $(2 n-4)$. Furthermore, notice that the set of "bad" geodesics, i.e. the ones that are tangent at some point to $\partial N_{x_{1}}^{\epsilon}$, is of dimension $(2 n-5)$ (we choose a point and a unit tangent direction). Let us now define (for $x_{1} \in T$ ):

$$
\Gamma_{x_{1}}=\left\{\text { geodesics } \gamma \in \Gamma \text { such that } \gamma \pitchfork \partial N_{x_{1}}^{\epsilon}\right\}
$$

and by the above dimension counting we have $\Gamma_{x_{1}}$ is of full measure in $\Gamma$. Let us consider the set

$$
A=\left\{\left(x_{1}, \gamma\right) \mid x_{1} \in T \text { and } \gamma \in \Gamma_{x_{1}}\right\} \subset J_{0} \times \Gamma
$$

Since $\Gamma_{x_{1}}$ is of full measure in $\Gamma$ and $T$ is of full measure in $J_{0}$, we have $A$ is of full measure in $J_{0} \times \Gamma$, by Fubini's theorem. Furthermore, again by Fubini's theorem applied to the indicator function $\chi_{A}$ of $A$, we conclude that for almost all $\gamma \in \Gamma$, the set $\left\{x_{1} \mid x_{1} \in J_{0}\right.$ and $\left.\gamma \in \Gamma_{x_{1}}\right\}$ is of full measure in $J_{0}$; let us denote the set of such $\gamma$ by $\Gamma^{\prime}$. This proves the claim, i.e. $\Gamma^{\prime}$ is of full measure in $\Gamma$.

Moreover, notice that if we take a countable set of $\epsilon$, say $\epsilon_{k} \rightarrow 0$ for $k \in \mathbb{N}$, then the set of geodesics that tranversely intersect $\partial N_{x_{1}}^{\epsilon_{i}}$ for a.a. $x_{1} \in J_{0}$ and all $i$ is of full measure, by taking a countable intersection.

We will also need the following claim: if $\gamma \in \Gamma^{\prime}$, then we have $\Phi_{i}^{\epsilon} \rightarrow \Phi_{i}$ uniformly in $\mathbb{R} \times[0, L]$ for $i=1,2$ as $\epsilon \rightarrow 0$. This follows from (7.16) and (7.18) in the polar coordinate form (the analogous argument works for $\Phi_{1}$ and $\Phi_{1}^{\epsilon}$ ):

$$
\left(\Phi_{2}^{\epsilon}-\Phi_{2}\right)(\omega)=\frac{1}{\pi} \int_{0}^{\infty} \int_{0}^{2 \pi}\left(\overline{Z_{2}^{\epsilon}}-\overline{Z_{2}}\right)\left(\omega+r e^{i \theta}\right) e^{-i \theta} d \theta d r
$$

Notice that the support of $Z_{2}^{\epsilon}-Z_{2}$ lies in the set $S_{\epsilon}:=\tilde{\gamma}^{-1}\left(N^{\epsilon}\right)$, where $\tilde{\gamma}: \mathbb{R} \times[0, L]$ maps $\left(x_{1}, t\right) \mapsto\left(x_{1}, \gamma(t)\right)$. So we may write

$$
\operatorname{supp}\left(Z_{2}^{\epsilon}-Z_{2}\right) \subset S_{\epsilon}=\bigcup_{x_{1} \in J_{0}}\left\{x_{1}\right\} \times \gamma^{-1}\left(N_{x_{1}}^{\epsilon}\right)
$$

Therefore, if we define $M=\left(\operatorname{supp}_{z, \epsilon}\left(\left|Z_{2}^{\epsilon}\right|\right)+\operatorname{supp}_{z}\left(\left|Z_{2}\right|\right)\right)$, we have the bound

$$
\left|\left(\Phi_{2}^{\epsilon}-\Phi_{2}\right)(\omega)\right| \leq \frac{M}{\pi} \int_{0}^{\infty} \int_{0}^{2 \pi} \chi_{S_{\epsilon}} d \theta d r \leq 2 r_{0} M+\frac{\operatorname{area}\left(S_{\epsilon}\right)}{r_{0}}
$$


for any $r_{0}>0$, where area $\left(S_{\epsilon}\right)$ is the 2-dimensional Lebesgue measure. But by (7.20), Fubini and the DCT, we have:

$$
\operatorname{area}\left(S_{\epsilon}\right)=\int_{x_{1}} \int_{\gamma^{-1}\left(N_{x_{1}}^{\epsilon}\right)} d t d x_{1} \rightarrow 0
$$

as $\epsilon \rightarrow 0$, since $\gamma \in \Gamma^{\prime}$. Therefore, by taking $r_{0}$ small enough and then taking $\epsilon$ small enough, (7.21) gives a small uniform bound, which proves the claim.

Back to the main proof, for $\gamma \in \Gamma^{\prime}$ we have

$$
\lim _{\tau \rightarrow \infty} \int_{N^{\epsilon}} \tilde{A}_{1}^{\epsilon} v_{1}^{\epsilon} \overline{v_{2}^{\epsilon}} d V_{\tilde{g}}=\lim _{\tau \rightarrow \infty} \int_{x_{1}} \int_{N_{x_{1}}^{\epsilon}} \tilde{A}_{1}^{\epsilon} v_{1}^{\epsilon} \overline{v_{2}^{\epsilon}} d V_{g_{0}} d x_{1}=\int_{x_{1}} \int_{\gamma^{-1}\left(N_{x_{1}}^{\epsilon}\right)} e^{\Phi_{1}^{\epsilon}+\bar{\Phi}_{2}^{\epsilon}} e^{-2 \lambda t} \tilde{A}_{1}^{\epsilon} d t d x_{1}
$$

by Fubini, the first observation above and the Dominated convergence theorem. We may apply the DCT as $\left\|v_{i}^{\epsilon}\right\|_{L^{2}\left(\left\{x_{1}\right\} \times M_{0}\right)}=O(1)$ as $\tau \rightarrow \infty$ uniformly in $x_{1} \in J_{0}$, for $i=1,2$. Furthermore, if we take $\epsilon=\epsilon_{k}$ with $\epsilon_{k} \rightarrow 0$ (e.g. $\epsilon_{k}=\frac{1}{k}$ for large enough $k$ ), we see that by the DCT (we drop the $k$ to lighten the notation):

$$
\lim _{\epsilon \rightarrow 0} \int_{x_{1}} \int_{\gamma^{-1}\left(N_{x_{1}}^{\epsilon}\right)} e^{\Phi_{1}^{\epsilon}+\overline{\Phi_{2}^{\epsilon}}} e^{-2 \lambda t} \tilde{A}_{1}^{\epsilon} d t d x_{1}=0
$$

since the length of $\gamma^{-1}\left(N_{x_{1}}^{\epsilon}\right)=o(1)$ as $\epsilon \rightarrow 0$, for a.a. $x_{1} \in J_{0}$ (as $\gamma \in \Gamma^{\prime}$ ) and the integrand is uniformly bounded. Analogously we obtain, by using Fubini, first observation and the DCT

$$
\begin{aligned}
\lim _{\tau \rightarrow \infty} \frac{1}{\tau} \int_{N^{\epsilon}} e^{-2 i \lambda x_{1}} \overline{v_{2}^{\epsilon}}\left\langle\tilde{A}^{\epsilon}, d v_{1}^{\epsilon}\right\rangle_{\tilde{g}} d V_{\tilde{g}}=\lim _{\tau \rightarrow \infty} \frac{1}{\tau} \int_{x_{1}} \int_{N_{x_{1}}^{\epsilon}} e^{-2 i \lambda x_{1}} \overline{v_{2}^{\epsilon}}\left\langle\tilde{A}^{\epsilon}, d v_{1}^{\epsilon}\right\rangle_{\tilde{g}} d V_{\tilde{g}} \\
=i \int_{x_{1}} \int_{\gamma^{-1}\left(N_{x_{1}}^{\epsilon}\right)} e^{-2 i \lambda x_{1}} \tilde{A}_{t}^{\epsilon} e^{\Phi_{1}^{\epsilon}+\bar{\Phi}_{2}^{\epsilon}} e^{-2 \lambda t} d t d x_{1}
\end{aligned}
$$

Note again that we may use the DCT as $\left\|d v_{i}^{\epsilon}\right\|_{L^{2}\left(\left\{x_{1}\right\} \times M_{0}\right)}=O(|\tau|)$ as $\tau \rightarrow \infty$ uniformly in $x_{1} \in J_{0}$, for $i=1,2$. If we now take $\epsilon_{k} \rightarrow 0$, for the same reasons as before, we get

$$
\lim _{\epsilon \rightarrow 0} i \int_{x_{1}} \int_{\gamma^{-1}\left(N_{x_{1}}^{\epsilon}\right)} e^{-2 i \lambda x_{1}} \tilde{A}_{t}^{\epsilon} e^{\Phi_{1}^{\epsilon}+\bar{\Phi}_{2}^{\epsilon}} e^{-2 \lambda t} d t d x_{1}=0
$$

Going back to the identity (7.13), taking $\tau \rightarrow \infty$ and combining with the two previous limits, we get:

$$
\int_{-\infty}^{\infty} \int_{0}^{L} e^{\Phi_{1}^{\epsilon}+\bar{\Phi}_{2}^{\epsilon}} e^{-2 \lambda t} e^{-2 i \lambda x_{1}}\left(\tilde{A}_{1}^{\epsilon}-i \tilde{A}_{t}^{\epsilon}\right) d t d x_{1}=o_{\epsilon}(1)
$$

where $o_{\epsilon}(1)$ means $o(1)$ as $\epsilon \rightarrow 0$. As before, by using Stokes' theorem and integrating by parts over a simply connected $\Omega \subset \mathbb{R} \times[0, L]$ that contains the supports of $Z_{i}^{\epsilon}$ for $i=1,2$, together with inserting an anti-holomorphic function $h$ (the estimates above go through with $h e^{\Phi_{1}^{\epsilon}}$ instead of $e^{\Phi_{1}^{\epsilon}}$, as $h$ is independent of $\epsilon$ ), we obtain

$$
\int_{\partial \Omega} e^{-2 i \lambda\left(x_{1}-i t\right)} h e^{\Phi_{1}^{\epsilon}+\overline{\Phi_{2}^{\epsilon}}} d \bar{z}=o_{\epsilon}(1)
$$

and so by taking the limit $\epsilon \rightarrow 0$

$$
\int_{\partial \Omega} e^{-2 i \lambda\left(x_{1}-i t\right)} h e^{\Phi_{1}+\overline{\Phi_{2}}} d \bar{z}=0
$$

Now we repeat the argument of taking the logarithm from the proof of Theorem 7.1 (c.f. 7.7) to get that

$$
\int_{\partial \Omega} e^{-2 i \lambda\left(x_{1}-i t\right)}\left(\Phi_{1}+\overline{\Phi_{2}}\right) d \bar{z}=0
$$


So by going back to the $\epsilon$ limit and integrating by parts, we get (c.f. 77.8 )

$$
\int_{\Omega} e^{2 i \lambda\left(x_{1}+i t\right)}\left(\tilde{A}_{1}^{\epsilon}+i \tilde{A}_{t}^{\epsilon}\right) d z d \bar{z}=o_{\epsilon}(1)
$$

Finally, by the Dominated convergence theorem we obtain

$$
\int_{0}^{L} e^{-\lambda r}(f+i \alpha(\dot{\gamma})) d r=0
$$

with rescaling $\lambda$ and where $f$ and $\alpha$ are defined by $(7.9)$ and $(7.10)$ as before, for geodesics $\gamma$ in $\Gamma^{\prime}$ (which is of full measure).

We claim that $f$ and $\alpha$ are in fact smooth. To show this, recall that the projection $\pi_{2}: \partial M \backslash$ $\Gamma \rightarrow M_{0}$ is a local diffeomorphism by definition of $\Gamma$ - therefore $\pi_{2}^{-1}\left(x^{\prime}\right)$ is a finite set of points for each $x^{\prime}$ that we denote by $b_{1}\left(x^{\prime}\right)<\ldots<b_{k}\left(x^{\prime}\right)$ locally. Furthermore we set $a_{1}\left(x^{\prime}\right)=-N$, and $a_{i}\left(x^{\prime}\right)=b_{i-1}\left(x^{\prime}\right)+\epsilon$ for $i \geq 2$ where $\epsilon^{\prime}>0$ small enough so that $\left(b_{i}\left(x^{\prime}\right), a_{i+1}\left(x^{\prime}\right)\right] \times\left\{x^{\prime}\right\} \subset M^{c}$ for $k-1 \geq i \geq 1$, where $M^{c}$ is the complement of $M$. Therefore

$$
f\left(\lambda, x^{\prime}\right)=\sum_{i=1}^{k} \int_{a_{i}\left(x^{\prime}\right)}^{b_{i}\left(x^{\prime}\right)} e^{i \lambda x_{1}} \tilde{A}_{1}\left(x_{1}, x^{\prime}\right) d x_{1}
$$

shows $f$ is smooth and similarly, so is $\alpha$. Same as before (formally), we get $\alpha=i d^{\prime} \beta$ and $f=\lambda \beta$ for some smooth $\beta$. By a computation and using $d^{\prime} \alpha=0$, we get

$$
\mathcal{F}\left(\partial_{j} \tilde{A}_{k}-\partial_{k} \tilde{A}_{j}\right)\left(\lambda, x^{\prime}\right)=\sum_{l=1}^{k} e^{i \lambda b_{l}\left(x^{\prime}\right)}\left(\frac{\partial b_{l}}{\partial x^{k}}\left(x^{\prime}\right) \tilde{A}_{j}\left(b_{l}\left(x^{\prime}\right), x^{\prime}\right)-\frac{\partial b_{l}}{\partial x^{j}}\left(x^{\prime}\right) \tilde{A}_{k}\left(b_{l}\left(x^{\prime}\right), x^{\prime}\right)\right)
$$

for $j, k \geq 2$ and all $x^{\prime}$ in a small open set and all $\lambda$. Note that the right hand side for fixed $x^{\prime}$ is in $L^{2}(\mathbb{R})$ if and only if the coefficients are zero; this implies that $\partial_{j} \tilde{A}_{k}=\partial_{k} \tilde{A}_{j}$ for a.a. $x_{1}$ and so $d^{\prime} \tilde{A}=0$ in $M$ by continuity.

Finally, by another computation and using $d^{\prime} f+i \lambda \alpha=0$, we have

$$
\mathcal{F}\left(\partial_{j} \tilde{A}_{1}-\partial_{1} \tilde{A}_{j}\right)\left(\lambda, x^{\prime}\right)=-\sum_{l=1}^{k} e^{i \lambda b_{l}\left(x^{\prime}\right)}\left(\frac{\partial b_{l}}{\partial x^{j}}\left(x^{\prime}\right) \tilde{A}_{1}\left(b_{l}\left(x^{\prime}\right), x^{\prime}\right)+\tilde{A}_{j}\left(b_{l}\left(x^{\prime}\right), x^{\prime}\right)\right)
$$

and we similarly conclude $\partial_{j} \tilde{A}_{1}=\partial_{1} \tilde{A}_{j}$ in $M$. Therefore, we globally have $d \tilde{A}=0$.

REMARK 7.3. In the case of a topologically non-trivial line bundle $E$, we can follow the lines of the proofs of Theorems 4.4, 7.1 and 7.2 to get that $d\left(A_{1}-A_{2}\right)=0$ (note that End $\mathrm{E}=E \otimes E^{*}$ in this case is a trivial bundle, since we have the identity section, so $A_{1}-A_{2}$ is a proper 1-form on $M$ ). Namely, what one can do is to take the partition of unity used in the construction of the CGOs subordinate to $V_{i}$ s and $W_{j}$ s (see the equations (4.9) and the paragraph below it); now in each of these charts we may trivialise the bundle and by essentially re-running the last part of Theorem 4.4 and Remark 4.9 dealing with the concentration properties, we get the limit of each individual term in the partition of unity; summing over again, we obtain the desired limit - the equation (7.6). Then the rest of the proof of Theorem 7.1 applies and we have a similar situation with Theorem 7.2 .

REMARK 7.4. We have proved that Cauchy data uniquely determines $d A$, however ideally we would like to determine the connection up to gauge equivalence, which is finer than just determining $d A$. On simply-connected manifolds, we would have $A_{2}-A_{1}=d p=e^{-p} d\left(e^{p}\right)$ for some $p$ that we may arrange to vanish on one component of the boundary - assuming the potentials are equal (or zero), the argument in Proposition 6.12 would imply that $e^{p} \equiv 1$ on the whole of $\partial M$. If additionally $\partial M$ is connected, we may recover a scalar potential, too (once we 
gauge transform one connection to the other, this would follow from the proof of Theorem 1.2 from [23]). However, we can make the case without the potentials even on non simply-connected manifolds; the proof is contained in the next section and the idea is to consider $A_{2}-A_{1}$ as a flat connection and to use a unique continuation principle.

\subsection{The holonomy argument}

Given a manifold $M$ and a Hermitian vector bundle $E$ on it, equipped with a unitary connection $\nabla$, we can define the parallel transport along piecewise smooth curves in $M$, which is an isometry on the fibers. In particular, when this curve is a loop at a point $p$, we end up with an isometry of the fibre $E_{p}$, i.e. $P_{\gamma}: E_{p} \rightarrow E_{p}$ which preserves the Hermitian inner product. When $E=M \times \mathbb{C}^{m}$ with the standard structure, $P_{\gamma}$ is a unitary matrix. The holonomy group at $p$ is defined as:

$$
H_{p}(\nabla)=\left\{P_{\gamma}: E_{p} \rightarrow E_{p} \mid \gamma \text { a closed loop at } p\right\}
$$

This naturally defines a group and moreover satisfies $P_{\gamma \cdot \gamma^{\prime}}=P_{\gamma} \cdot P_{\gamma^{\prime}}$ under path concatenation. We can also define the restricted holonomy group as the group $H_{p}^{0}(\nabla)$ consisting of parallel transports along contractible loops - which yields a surjective homomorphism $\rho_{p}^{\nabla}: \pi_{1}(M, p) \rightarrow$ $H_{p}(\nabla) / H_{p}^{0}(\nabla)$ called the holonomy representation. On a fixed connected manifold, these groups for varying points are all isomorphic upon conjugation by an appropriate element.

There is a close connection between the holonomy and the curvature. Namely, one can say that "the curvature is an infinitesimal of the deviation of the holonomy"; more concretely, if we are given a parallelogram in a coordinate chart determined by two coordinate axes, say $x_{1}$ and $x_{2}$, then $F_{12} u=-\frac{\partial^{2}}{\partial s \partial t} T_{s, t} u$, where $F_{12}$ is the corresponding component of the curvature tensor and $T_{s, t}$ is the parallel transport along parallelogram at vertices $(0,0),(s, 0),(s, t),(0, t)$. For our purposes, we will need the fact that homotopic paths have the same holonomy if the curvature is zero.

Lemma 7.5. If the curvature $F_{\nabla}$ of $\nabla$ is zero, then $H_{p}^{0}(M)=0$ for all $p \in M$.

Proof. Let $\sigma: I \times I \rightarrow M$ be a smooth homotopy between a loop $\gamma$ and the constant loop at $p \in M$, fixing the endpoints. We will make use of the identity:

$$
\nabla_{\frac{\partial \sigma}{\partial x}} \nabla_{\frac{\partial \sigma}{\partial y}} V-\nabla_{\frac{\partial \sigma}{\partial y}} \nabla_{\frac{\partial \sigma}{\partial x}} V=F_{\nabla}\left(\frac{\partial \sigma}{\partial x}, \frac{\partial \sigma}{\partial y}\right) V
$$

where $V$ is any section. Let us put $V_{x, t}=T_{x, t} v$ for some $v \in E_{p}$, where $T_{x, t}$ is parallel transport along $\sigma(x, \cdot)$; also $\sigma(0, t)=p$ and $\sigma(1, t)=\gamma(t)$. Then we must have $0=\nabla_{\frac{\partial \sigma}{\partial x}} \nabla_{\frac{\partial \sigma}{\partial t}} V_{x, t}=$ $\nabla_{\frac{\partial \sigma}{\partial t}} \nabla_{\frac{\partial \sigma}{\partial x}} V_{x, t}$, which implies that $\nabla_{\frac{\partial \sigma}{\partial x}} V_{x, t}$ is parallel along $\sigma(x, \cdot)$ for all $x$. But $V_{x, 0} \stackrel{\frac{\partial \sigma}{\partial t}}{=} v$ and $\sigma(x, 0)=p$ for all $x$ and so we have $\nabla_{\frac{\partial \sigma}{\partial x}} V_{x, 0}=0$ for all $x$. By uniqueness of solution, we

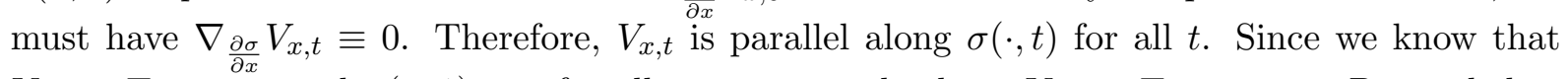
$V_{0,1}=T_{0,1} v \stackrel{\partial x}{=} v$ and $\sigma(x, 1)=p$ for all $x$, we must also have $V_{1,1}=T_{1,1} v=v=P_{\gamma} v$ and thus parallel transport along $\gamma$ is trivial.

This means that for zero curvature, the holonomy representation is simply a map from $\pi_{1}$ to the holonomy group. As a warm up, let us point out some details about the construction of the parallel transport matrix. Namely, assume $E=M \times \mathbb{C}^{m}$ with a unitary connection $A$ has trivial holonomy and fix a point $p \in M$. Consider the matrix obtained by parallel transporting along curves emanating from $p$ and define $F\left(p^{\prime}\right)=P_{\gamma\left(p, p^{\prime}\right)}$ where $\gamma\left(p, p^{\prime}\right)$ is a path between $p$ and $p^{\prime}$. Since the holonomy is trivial, we have $F$ well defined. Therefore, we have $d F+A F=0$ for all $(x, v) \in T M$ and also $F F^{*}=I d$, since $A$ is unitary. Hence $F^{-1} A F+F^{-1} d F=0$ 
and so $A$ is equivalent to the trivial connection and moreover the covariant derivative satisfies $F^{-1}(d+A) F=d$. Moreover, if we fix $p \in \partial M$ and assume that $\iota_{\Gamma}^{*} A=0$ for a connected open set $\Gamma \subset \partial M$, we will have $\left.F\right|_{\Gamma}=I d$, so $A$ and the trivial connection on $E$ will be gauge equivalent.

It is useful to note that when the vector bundle $E$ is trivial, we may apply Lemma 2.4 to transfer the covariant normal derivative to the usual one, because most results on unique continuation for elliptic systems work with usual normal derivative at the boundary, as in [39 or 25 (c.f. Remark 7.8 below). In the next result, we will use the boundary determination result Theorem 3.4 .

THEOREM 7.6. Let $E$ be a Hermitian vector bundle, equipped with two flat, unitary connections $A$ and $B$, and $\Gamma$ an open, non-empty subset of the boundary $\partial M$. Then the restricted $D N$ maps agree, i.e. $\left.\Lambda_{A}\right|_{\Gamma}(f)=\left.\Lambda_{B}\right|_{\Gamma}(f)$ for all $f \in C_{0}^{\infty}\left(\Gamma ;\left.E\right|_{\Gamma}\right)$ if and only if $\iota_{\Gamma}^{*}(A-B)=0$, the holonomy representations satisfy $\rho^{A}=\rho^{B}$ and the parallel transport matrices along any path with endpoints in $\Gamma$, with respect to $A$ and $B$ are equa!.

Proof. Let us firstly assume $\Lambda_{A}=\Lambda_{B}$ on $C_{0}^{\infty}\left(\Gamma ;\left.E\right|_{\Gamma}\right)$. We know this implies by boundary determination that $\iota_{\Gamma}^{*}(B-A)=0$. Consider $p_{1} \in \Gamma$ and a loop $\gamma$ starting at $p_{1}$. By standard differential topology, we can always homotopically perturb the curve such that we end up with two pieces of it: $\gamma_{1}:[0,1] \rightarrow M$ starting at $p_{1}$ and ending at $p_{2} \neq p_{1} \in \Gamma$ such that $\gamma(0,1) \subset$ int $M$; and $\gamma_{2}:[1,2] \rightarrow M$ starting at $p_{2}$ and ending at $p_{1}$ and Image $\left(\gamma_{2}\right) \subset \Gamma$. We moreover ask that $\gamma_{1}$ and $\gamma_{2}$ are embedded curves 2 . In order to show that the holonomies are equal, it suffices to show the parallel transports along $\gamma_{1}$ are equal, as $\iota_{\Gamma}^{*}(B-A)=0$.

We consider a tubular neighbourhood of $\gamma_{1}$; every such is of form $\mathcal{O}=\left\{p \in M^{\circ} \mid \operatorname{dist}\left(p, \gamma_{1}\right)<\right.$ $\epsilon\} \cong(0,1) \times B_{\epsilon}(0)$, where $B_{\epsilon}(0)$ is an $(n-1)$-dimensional ball (every vector bundle over a contractible space is trivial). Therefore, we know $\mathcal{O}$ is simply connected and therefore has trivial holonomy $H_{p_{1}}\left(\mathcal{O},\left.B\right|_{\mathcal{O}}\right)=\{0\}$; here we also used that $B$ is flat and similarly for $A$. We consider $\epsilon>0$ such that $\operatorname{dist}\left(p_{1}, p_{2}\right)>2 \epsilon$, so that we have a cylindrical neighbourhood with disjoint ends. Denote $U_{1}=\left\{p \in \Gamma: \operatorname{dist}\left(p, p_{1}\right)<\epsilon\right\}$.

Now since both connections are flat and $\mathcal{O}$ is simply-connected, we get a unitary isomorphism $F$ between them: $F$ is obtained by taking parallel transport matrices from $p$ of both connections and composing them in a suitable way. We also have $\left.F\right|_{U_{1}}=I d$, as $\iota_{\Gamma}^{*}(B-A)=0$. Now we apply the hypothesis on the DN maps - let $u_{1}$ and $u_{2}$ solve $\mathcal{L}_{A} u_{1}=\mathcal{L}_{B} u_{2}=0$ with same boundary data $\left.u_{1}\right|_{\Gamma}=\left.u_{2}\right|_{\Gamma}=f$, so that $\left.\nabla_{\nu}^{A}\left(u_{1}\right)\right|_{\Gamma}=\left.\nabla_{\nu}^{B}\left(u_{2}\right)\right|_{\Gamma} ;$ here $\mathcal{L}_{A}=\nabla_{A}^{*} \nabla_{A}$ and $\mathcal{L}_{B}=\nabla_{B}^{*} \nabla_{B}$. Define $u:=F u_{1}$ in $\mathcal{O}$; we want to prove that $u=u_{2}$ on $\mathcal{O}$.

Since $\left.F\right|_{U_{1}}=I d$, we have $\left.u\right|_{U_{1}}=\left.u_{1}\right|_{U_{1}}=\left.u_{2}\right|_{U_{1}}=\left.f\right|_{U_{1}}$. Also, using the introduction to this section and the definition of $F$, we have $F^{*} \nabla_{B} F=\nabla_{A}$ in $\mathcal{O}$ ( $F$ is unitary). Therefore, we must have:

$$
\mathcal{L}_{A}=F^{*} \nabla_{B}^{*} F F^{*} \nabla_{B} F=F^{*} \mathcal{L}_{B} F
$$

which implies that $\mathcal{L}_{B} u=0$ in $\mathcal{O}$. Moreover, we have $\nabla_{\nu}^{B} u=\nabla_{\nu}^{B} u_{2}$ on $U_{1}$ :

$$
\begin{aligned}
\nabla_{\nu}^{B} u & =\left(d F u_{1}+F d u_{1}\right)(\nu)+B(\nu) u=(F A(\nu)-B(\nu) F) u_{1}+B(\nu) u+F \partial_{\nu} u_{1} \\
& =\partial_{\nu} u_{1}+A(\nu) u_{1}=\nabla_{\nu}^{A} u_{1}=\nabla_{\nu}^{B} u_{2}
\end{aligned}
$$

\footnotetext{
${ }^{1}$ More precisely, given any $x, x^{\prime} \in \Gamma$ and any path $\gamma$ between them, the parallel transport matrices $F, G: E_{x} \rightarrow E_{x^{\prime}}$ with respect to $A$ and $B$ (respectively) along $\gamma$ are equal, i.e. $F=G$. This is to address the case when $\Gamma$ is potentially disconnected.

${ }^{2}$ We can always do this for curves in dimension $n \geq 3$ by using a version of the weak Whitney theorem to approximate; then we apply a result which says when we are close to a curve uniformly, we are homotopic to it for the case $n=2$ see Remark 7.9
} 
Consequently, we have:

$$
\mathcal{L}_{B}\left(u-u_{2}\right)=0,\left.\quad\left(u-u_{2}\right)\right|_{U_{1}}=0 \quad \text { and }\left.\quad \nabla_{\nu}^{B}\right|_{U_{1}}\left(u-u_{2}\right)=0
$$

so by a result concerning the unique continuation properties of elliptic systems of equations (see Remark 7.8 below), we must have $u \equiv u_{2}$ in $\mathcal{O}$; hence we must also have equality at $p_{2}$ by letting $p \in \mathcal{O}$ converge to $p_{2}$, i.e. $F\left(p_{2}\right) f\left(p_{2}\right)=f\left(p_{2}\right)$. Here $f$ is smooth and free to choose and therefore, we must have $F\left(p_{2}\right)=I d$. This concludes the proof that the holonomies are equal.

The same proof as above shows that given any $p_{1}, p_{2} \in \Gamma$ and a path $\gamma$ between them, the parallel transport matrices along $\gamma$ of $A$ and $B$ agree, i.e. in the above notation we have $F\left(p_{2}\right)=I d$.

Conversely, to show that $A$ and $B$ have the same restricted DN maps under the given assumptions, just follow the paragraph before the theorem ( $B=0$ case); however, note that since we do not know that the holonomy is trivial, parallel transport from a point might not be well-defined, so we have to do something else. The idea is to provide a global horizontal section of the endomorphism bundle that is identity at the boundary and relate this with holonomy.

Induce the standard unitary connection on the End $E$ bundle by $\nabla^{\text {End }} u=\nabla_{B} u-u \nabla_{A}$; one can easily check this new connection to be flat, as $A$ and $B$ are. Note that in a local trivialisation this is just $\hat{A}(R)=B R-R A$, where $\hat{A}$ is the new connection matrix and $R$ is a matrix. We would like to construct a unitary automorphism $U$ of $E$, such that $\left.U\right|_{\Gamma}=I d$ and $U^{*} \nabla_{A} U=\nabla_{B}$. We do this as follows.

Fix $p_{1} \in \Gamma$ as before and take a loop at $p_{1}$, homotope it as before and assume we are working in the tubular neighborhood $\mathcal{O}$. Then $A$ and $B$ are equivalent to a trivial connection over $\mathcal{O}$; take the parallel transport matrices $F$ and $G$ such that $d F+A F=d G+B G=0$ in $\mathcal{O}$. Then one checks for $H=G F^{-1}$ :

$$
d H=d G F^{-1}+G d F^{-1}=-B G F^{-1}+G F^{-1} A=H A-B H
$$

One also sees that $\left.H\right|_{U_{1}}=I d$ as $\iota_{\Gamma}^{*}(A-B)=0$; also, as $\rho_{A}=\rho_{B}$ and the parallel transport along paths in $\Gamma$ is the same for $A$ and $B$, we have that $\left.H\right|_{U_{2}}=I d$, too. Now as $H\left(\gamma_{1}(t)\right)$ is parallel transport with respect to $\hat{A}$, we get the parallel transport of $I d$ along $\gamma_{1}$ at $p_{2}$ is $I d$; therefore parallel transport of $I d$ along $\gamma$ is trivial. So we may define $U(x)$ to be parallel transport with respect to $\hat{A}$ of $I d$ from $p_{1}$ to $x$, for every $x \in M$; the fact we get identity when we parallel transport between any two components at the boundary then gives $\left.U\right|_{\Gamma}=I d$, which concludes the proof.

Note that the proof above does not generalise if we add arbitrary potentials, since the local gauge isomorphism between two connections has no a priori reason to intertwine the potentials (see $(7.22)$ ). However, it generalises in the case $m=1$ and $Q_{A}=Q_{B}$, since the group action is abelian in that case.

REMARK 7.7. Moreover, in the case of line bundles, it is true that for any two connections $A_{1}$ and $A_{2}$ for which we know $d\left(A_{1}-A_{2}\right)=0:\left.\Lambda_{A_{1}}\right|_{\Gamma}=\left.\Lambda_{A_{2}}\right|_{\Gamma}$ if and only if $\iota_{\Gamma}^{*}\left(A_{1}-A_{2}\right)=0$, the holonomy representation of $A_{1}-A_{2}$ (on $M \times \mathbb{C}$ ) is trivial and the parallel transport maps with respect to $A_{1}-A_{2}$ between boundary components in $\Gamma$ are equal to the identity. This can be easily seen from the above proof.

REMARK 7.8. The unique continuation result we are using follows from Theorem 2.3 in [44, which considers the case of the wave equation (covers our setting if we let $u$ independent of $t$ ) with the covariant normal derivative at the boundary and so solves our problem. However, it is 
not ideal since it gives more than we need. More adequate are techniques in Corollary 3.4 and Theorem 3.2 in 25 (although they do not use the covariant derivative), since for an elliptic operator, any smooth surface is pseudoconvex. See also Appendix B in $[\mathbf{3 9}]$.

REMARK 7.9. In the case of surfaces, we need to be careful when approximating curves by embeddings - we do not have enough space to get rid of possible self-intersections. However, there is a way around this by considering just the class of simple curves, by which we can represent generators of $\pi_{1}$ (see $\mathbf{3 6}$, Section 6, for details). Furthermore, in [36 the Conjecture $\mathrm{A}$ for Riemann surfaces and line bundles is proved, but with the extra bit of a potential added to the connection Laplacian (so the claim is more general in that case). There, the authors prove the identification of a potential before identification of a connection (see the comment after the proof of Theorem 7.6. In our recovery Theorem 7.1, we first prove the identification of a connection.

Now we are in a good shape to prove the main theorem: all ingredients are ready. Theorems 7.1 and 7.2 almost finish the proof, however Theorem 7.6 provides us with the necessary gauge.

Proof of Theorem E. Recall that we have $d\left(A_{1}-A_{2}\right)=0$ from Theorem 7.1 for full data and from Theorem 7.2 for partial data. By Remark 7.7, we immediately get our gauge in both cases. This finishes the proof. 



\section{CHAPTER 8}

\section{Proof of the Main Theorem II}

In this chapter we consider the main conjecture in the special case of Yang-Mills connections. Moreover, we prove our two main results in this setting: Theorem $\mathrm{G}$ for line bundles in the smooth category and Theorem $[\mathrm{H}$ for rank $m>1$ bundles in the analytic category. In the proofs, we introduce a new technique that we call "drilling", based on the unique continuation principles for elliptic systems - heuristically, the idea is to gauge transform a pair of connections using suitable gauges to a pair of connections that are singular over a countable union of hypersurfaces and apply the degenerate UCP possibly infinitely many times to "drill through" the hypersurfaces. The analytic assumption in the $m>1$ case is technical.

\subsection{Recovering a Yang-Mills connection}

We fix a Yang-Mills connection $A$ on the Hermitian vector bundle $E=M \times \mathbb{C}^{m}$ (with the standard metric) over a compact Riemannian manifold $(M, g)$ with boundary. Let us extend the connection $A$ to a "new connection" on the endomorphism bundle End $E=M \times \mathbb{C}^{m \times m}$ by simply asking that $d_{\tilde{A}} F=d F+A F$ globally, where $\tilde{A}$ is the matrix of one forms with values in $\operatorname{End}\left(\mathbb{C}^{m \times m}\right)$ induced by $A$ by multiplication on the left; it is easy to check this is a unitary connection. Note that $d_{\tilde{A}}$ does not satisfy the usual Leibnitz rule such does the usual connection $D_{A} F=d F+[A, F]$ on the endomorphism bundle. Recall that the DN maps associated to the vector bundle $E$ and operators $\mathcal{L}_{A}$ and $\mathcal{L}_{B}$ are equal if and only if they agree for the induced operators $\mathcal{L}_{\tilde{A}}$ and $\mathcal{L}_{\tilde{B}}$ on the endomorphism bundle. Here and throughout the chapter, we will use the same notation $d_{A}=d+A$ for both covariant derivatives $d_{A}$ and $d_{\tilde{A}}$, which will hopefully be clear from context. We will also use $d_{A}$ to denote the extension of the covariant derivative to vector valued forms. The complex bilinear form on matrix valued 1 -forms $(\alpha, \beta)=g^{i j} \alpha_{i} \beta_{j}$ is obtained by extending the usual inner product on forms. We start by writing down a simple, but key lemma that will yield the right gauge in our situation:

Lemma 8.1. If $U \subset \mathbb{R}^{n}$ open and $F: U \rightarrow \mathbb{C}^{m \times m}$ is an invertible matrix function and we put $A^{\prime}=F^{*}(A)$ for $A$ a matrix of one forms on $U$, then $F$ satisfies $d_{A}^{*} d_{A} F=0$ if and only if $d^{*} A^{\prime}=Q_{0}\left(x, A^{\prime}\right)$, where $Q_{0}$ is smooth of order zero and quadratic in $A^{\prime}$, given by $Q_{0}\left(x, A^{\prime}\right)=\left(A^{\prime}, A^{\prime}\right)$. If in addition $A$ is Yang-Mills, then $A^{\prime}$ satisfies an elliptic non-linear equation with diagonal principal part.

Proof. By using that $d_{A^{\prime}}=F^{-1} d_{A} F$ and similarly $d_{A^{\prime}}^{*}=F^{-1} d_{A}^{*} F$ (note this might not be the true formal adjoint - see preliminaries), note that $d_{A}^{*} d_{A} F=0$ is equivalent to the following:

$$
F F^{-1} d_{A}^{*} F F^{-1} d_{A} F=0 \Longleftrightarrow F d_{A^{\prime}}^{*} d_{A^{\prime}}(I d)=0 \Longleftrightarrow d^{*} A^{\prime}=\left(A^{\prime}, A^{\prime}\right) \Longleftrightarrow d_{A^{\prime}}^{*} A^{\prime}=0
$$

by expanding the $d_{A^{\prime}}^{*} d_{A^{\prime}}$ operator by (2.6). If $A$ is Yang-Mills, then by adding $\left(D_{A^{\prime}}\right)^{*} F_{A^{\prime}}=0$ to $d d^{*} A^{\prime}=d\left(Q_{0}\left(x, A^{\prime}\right)\right)$ we get an elliptic system with principal part equal to $d d^{*}+d^{*} d$.

By standard elliptic theory and the fact that $\operatorname{ker}\left(d_{A}^{*} d_{A}\right)=\{0\}$, we know that we may solve $d_{A}^{*} d_{A} F=0$ in $H^{1}\left(M ; \mathbb{C}^{m \times m}\right)$ uniquely for any boundary condition in $H^{\frac{1}{2}}\left(\partial M ; \mathbb{C}^{m \times m}\right)$. 
Therefore, at least near the boundary, we know that $A^{\prime}$ exists if $\left.F\right|_{\partial M}$ is smooth non-singular and that it satisfies the equation $d^{*} A^{\prime}=Q_{0}\left(x, A^{\prime}\right)$. Thus we may obtain the following result:

Theorem 8.2. Consider two Yang-Mills connections $A$ and $B$ on $E=M \times \mathbb{C}^{m}$ with the same DN map on the whole of $\partial M$. Then there exists a neighbourhood $U$ of the boundary and a bundle isomorphism $H$ for the restricted bundle $\left.E\right|_{U}$ with $\left.H\right|_{\partial M}=I d$ such that $H^{*} B=A$ on $U$. Moreover, if $A$ and $B$ are unitary (with respect to the standard structure), then we have $H$ to be a unitary automorphism.

Proof. By the construction above, we obtain smooth gauge equivalences $F$ and $G$, which satisfy $d_{A}^{*} d_{A} F=0$ and $d_{B}^{*} d_{B} G=0$ respectively, with boundary conditions $\left.F\right|_{\partial M}=\left.G\right|_{\partial M}=I d$. This is non-singular near the boundary and the connections $A^{\prime}=F^{*}(A)$ and $B^{\prime}=G^{*}(B)$ satisfy the equations

$$
d^{*} A^{\prime}=Q_{0}\left(x, A^{\prime}\right) \quad \text { and } \quad d^{*} B^{\prime}=Q_{0}\left(x, B^{\prime}\right)
$$

Now we can also expand the equations $\left(D_{A^{\prime}}\right)^{*} F_{A^{\prime}}=0=\left(D_{B^{\prime}}\right)^{*} F_{B^{\prime}}$ (note that $A^{\prime}$ and $B^{\prime}$ are now Yang-Mills with respect to the fibrewise inner product pulled back by $F$ and $G$ respectively, rather than the standard inner product):

$$
\left(d^{*} d+P\right) A^{\prime}=0 \quad \text { and } \quad\left(d^{*} d+P\right) B^{\prime}=0
$$

where $P$ is a first order, non-linear operator arising from the equality

$$
\left(d^{*} d+P\right) A^{\prime}=(-1)^{n+1} \star D_{A^{\prime}} \star F_{A^{\prime}}
$$

where $\star$ is the Hodge star extended to bundle valued forms. Therefore by simply applying the operator $d$ to (8.1) and adding to the Yang-Mills equations, we obtain an elliptic system of equations, with diagonal principal part

$$
\Delta A^{\prime}=\left(d d^{*}+d^{*} d\right) A^{\prime}=Q_{1}\left(x, A^{\prime}, \nabla A^{\prime}\right)
$$

where $Q_{1}$ is a smooth term of first order, polynomial in $A^{\prime}$ and $\nabla A^{\prime}$. In order to use uniqueness of solutions to such equations, we need some boundary conditions - this is where we use the DN map hypothesis. Without loss of generality, assume that the normal components of connections $A$ and $B$ near the boundary vanish (see Lemma 2.4).

Thus from equality of the DN maps, we have $\left.\frac{\partial(F-G)}{\partial \nu}\right|_{\partial M}=0$. By subtracting the initial equations for $F$ and $G$, we get:

$$
\Delta(F-G)-2(A, d F)+2(B, d G)+\left(d^{*} A\right) F-\left(d^{*} B\right) G-(A, A F)+(B, B G)=0
$$

and the point is that we have $\Delta(F-G)$ equal to lower order terms, where we are fixing the semi-geodesic boundary coordinates $(x, t)$ with $t$ denoting the direction of the normal - this is because we already know that $(A-B)=O\left(t^{\infty}\right)$, if $n \geq 3$, by the boundary determination result Theorem 3.4 , and $(F-G)=O(t)$. Thus when expanding the Laplacian, we are left with only $\frac{\partial^{2}}{\partial t^{2}}$ factor, which then allows us to conclude inductively $(F-G)=O\left(t^{\infty}\right)$ by repeated differentiation.

If $n=2$, notice that by Remark 3.5 we have $(A-B)=O(t)$; by 8.3 we have $(F-G)=O\left(t^{2}\right)$ and thus we have also that $\left(A^{\prime}-B^{\prime}\right)=O(t)$. Therefore by the elliptic equation 8.2$)$, the analogous counterpart of it for $B^{\prime}$ and repeated differentiation we obtain $\left(A^{\prime}-B^{\prime}\right)=O\left(t^{\infty}\right)$.

Therefore, we are left with two connections $A^{\prime}$ and $B^{\prime}$ which satisfy an elliptic equation and have the same full Taylor series at the boundary - by the unique continuation property for 
elliptic systems with diagonal principal part (see Remark 7.8 ), we may conclude $A^{\prime} \equiv B^{\prime}$ in $U$ and hence if we put $H=G F^{-1}$ we have $H^{*} B=A$ on $U$.

Finally, if $A$ and $B$ are unitary, we have that (locally, in a unitary trivialisation) $H^{*}(A)=B$ implies by definition that $d H=H B-A H$ and $d\left(H^{*}\right)=-B H^{*}+H^{*} A$, by the unitary property of connection matrices - combining the two, we have:

$$
d\left(H H^{*}\right)=\left[H H^{*}, A\right]
$$

where $[\cdot, \cdot]$ is the commutator. This first order system has a unique solution, which is given by $H H^{*}=I d$, as $\left.H\right|_{\partial M}=I d$ and thus $H$ is unitary whenever $H^{*}(A)=B$.

The next step is to go inside the manifold from the boundary. Namely, the main problem lies in the fact that $F$ can be singular on a large set, stopping our argument of unique continuation. However, at least in the scalar case, we may get over this, by essentially knowing facts about zero sets of solutions to elliptic systems of equations. We need to recall the following definition:

Definition 8.3. A subset of a smooth manifold is called countably $k$-rectifiable if it is contained in a countable union of smooth $k$-dimensional submanifolds.

The result we will need is essentially proved in [7], Theorem 2, for the scalar case; the vector case we will need follows in a straightforward manner from its method of proof. We outline it here for completeness.

Lemma 8.4. Let $\left(M_{0}, g_{0}\right)$ be a smooth n-dimensional, connected Riemannian manifold. Let $L: C^{\infty}\left(M_{0}, \mathbb{R}^{l}\right) \rightarrow C^{\infty}\left(M_{0}, \mathbb{R}^{l}\right)$ be a differential operator on vector functions for $l$ a positive integer, such that:

$$
L u(x)=\Delta u(x)+R(x, u(x), d u(x))
$$

where $\Delta$ is the metric Laplacian, $R$ is a smooth function with values in $\mathbb{R}^{l}$. Moreover, we assume that $R$ respects the zero section, i.e. $R(x, 0,0)=0$.

Now assume $u \not \equiv 0$ is a solution to Lu=0. Let us denote $\mathcal{N}(u)=u^{-1}(0)$ the zero set and by $\mathcal{N}_{\text {crit }}(u)=\mathcal{N}(u) \cap\{x \mid d u(x)=0\}$ the critical zero set. Then we claim that $\mathcal{N}(u)$ is countably $(n-1)$-rectifiable and moreover, $\mathcal{N}_{\text {crit }}(u)$ is countably $(n-2)$-rectifiable.

Proof. Consider the vector bundle $E_{0}=\bigoplus_{j}\left(\Lambda^{j} T^{*} M_{0} \otimes \mathbb{R}^{l}\right)$ of vector valued differential forms. It is a well known fact that the operator $d+\delta$ is a Dirac operator on the bundle of differential forms with respect to the Riemannian inner product (it respects the Clifford relations), where $\delta$ is the codifferential. Moreover, we have that $(d+\delta)^{2}=d \delta+\delta d=\Delta$ on differential forms. Let us consider the operator:

$$
V\left(\sum \omega_{i}\right)=R\left(x, \omega_{0}, \omega_{1}\right)-\omega_{1}
$$

where $\omega_{i}$ is the component of $\omega$ in $\Lambda^{i} T^{*} M_{0} \otimes \mathbb{R}^{l}$. Clearly $V$ is smooth and respects the zero section.

Thus, if $L u=0$, then $\omega=u+d u \in C^{\infty}\left(M ; E_{0}\right)$ solves $(d+\delta+V)(\omega)=0$. The first order operator $D=d+\delta+V$ is a Dirac operator acting on sections of $E_{0}$, so the Corollary 1 of 7 applies (the strong unique continuation property holds for a Dirac operator, i.e. we cannot have a non-zero solution vanishing to an infinite order at a point). Thus we get the result for the $\mathcal{N}_{\text {crit }}(u)=\mathcal{N}(\omega)$.

Finally, since $D$ has the SUCP, we know that $\mathcal{N}(u)$ consists of points where $u$ vanishes to finite order and hence the Lemma 3 from $[7]$ applies. 
We are now ready to prove the main theorem:

Proof of Theorem G. Firstly, gauge transform both $A$ and $B$ such that the normal component of the connection near the boundary is zero (apply Lemma 2.4). Consider the gauge constructed in Theorem 8.2 , i.e. $d_{A}^{*} d_{A} f=0$ and $d_{B}^{*} d_{B} g=0$ with the following boundary conditions: $\left.f\right|_{\partial M}=\left.g\right|_{\partial M},\left.f\right|_{V}=\left.g\right|_{V}=1$ and $f, g$ have compact support at the boundary contained in $\Gamma$. Here $V \subset \bar{V} \subset \Gamma$ is some non-empty, connected, open subset of $\Gamma]^{1}$ Let us define $h=\frac{f}{g}$ on the complement of the closed set $\mathcal{N}(g)=g^{-1}(0)$. We furthermore split the zero set into the critical set $\mathcal{N}_{\text {crit }}(g)=\mathcal{N}(g) \cap\{x \in M \mid d g(x)=0\}$ and its complement in $\mathcal{N}(g)$, $S=\mathcal{N}(g) \cap\{x \in M \mid d g(x) \neq 0\}$.

Now we consider the connections $A^{\prime}=f^{*}(A)$ and $B^{\prime}=g^{*}(B)$ near the set $V$, where we know $f$ and $g$ are non-zero, so these connections are well-defined. Following the recipe from before, by boundary determination and unique continuation we know that in a neighbourhood of $V$ in $M$, we have $A^{\prime} \equiv B^{\prime}$ and thus on this set we also have $B=h^{*}(A)$ or equivalently

$$
d h=(B-A) h
$$

Notice that $B=h^{*}(A)$ holds in the connected component $R$ of $V$ in the set $M \backslash \mathcal{N}(g) \cap M \backslash \mathcal{N}(f)$. Notice also that $d\left(|h|^{2}\right)=0$ on this component by using (8.4), since $A$ and $B$ are unitary, so $|h|$ is constant and hence bounded on this set. This implies that the zero sets of $f$ and $g$ agree as we approach the boundary of $R$. The problem now is how to go further inside the interior of the manifold and go past the zero sets of $f$ and $g$. We will do this by a procedure we call "drilling holes".

Let us describe this procedure. Firstly, we have that the zero set of $g$ lying in the interior of $M$ is contained in a countable union of codimension 1 submanifolds by Lemma 8.4; denote these manifolds by $M_{1}, M_{2}, \ldots$ Consider the following situation: we are given a point $p$ such that we have $g(p)=0$ and $d g(p) \neq 0$ and moreover, we have $g^{-1}(0)$ locally a hypersurface of codimension one (in this case the rank of $d g$ is equal to one). By going to a tubular neighbourhood of $g^{-1}(0)$ near $p$, we may assume we are in the setting where $g=0$ in a neighbourhood of zero in the hyperplane $\mathbb{R}^{n-1}$ and the metric satisfies $g_{i n}=\delta_{i n}$ for $i=1,2, \ldots, n$ in this coordinate system. Moreover, assume that we know $d h=h(B-A)$ or equivalently, that $f^{*}(A)=g^{*}(B)$, in the region where $\left\{x_{n}>0\right\}$. Our goal is to extend this equality to the lower part of the space.

Let us just remark that, in general, the zero set of $g$ can be of codimension one or two, depending on the rank of $d g$; however, if $d g \neq 0$ we anyway know that at least one of $d(\operatorname{Im} g) \neq 0$ and $d(\operatorname{Re} g) \neq 0$ holds, so the zero set is locally contained in $(\operatorname{Im} g)^{-1}(0)$ and $(\operatorname{Re} g)^{-1}(0)$, at least one of which is a codimension one submanifold. It can of course happen that the zero of $g$ contains an $(n-1)$-dimensional submanifold, see Figure 1 below for such an example (more precisely, $u$ in this example gives the real part of such a solution, with the imaginary part equal to zero).

By Taylor's theorem we have that $f=x_{n} f_{1}$ and $g=x_{n} g_{1}$ locally near 0 . Furthermore, $g_{1} \neq 0$ in a neighbourhood of 0 by the assumption and hence $f_{1} \neq 0$ as $|h|$ is a non-zero constant in the upper space. We want to consider $A^{\prime}=f^{*}(A)$ as before, however $f$ can be zero now and thus $A^{\prime}$ not well-defined (singular), so we will consider something very similar, i.e. $A^{\prime \prime}=x_{n} A^{\prime}$

\footnotetext{
${ }^{1}$ We will actually see later that it is enough to have any $f$ and $g$ non-zero and equal at the boundary.
} 
and $B^{\prime \prime}=x_{n} B^{\prime}$

$$
\begin{aligned}
& A^{\prime \prime}=d x_{n}+x_{n} \frac{d f_{1}}{f_{1}}+x_{n} A \\
& B^{\prime \prime}=d x_{n}+x_{n} \frac{d g_{1}}{g_{1}}+x_{n} B
\end{aligned}
$$

Now both of these are well-defined and the degeneracies have cancelled with $x_{n}$. Let us rewrite the gauge equations for $A^{\prime \prime}$ (note that $A^{\prime}$ is Yang-Mills with respect to pullback inner product by $f^{*}$ and not the standard inner product structure) as follows:

$$
\begin{aligned}
x_{n}^{2} d^{*} d\left(A^{\prime \prime}\right)+x_{n}\left(\iota \nabla x_{n} d A^{\prime \prime}-d^{*}\left(d x_{n} \wedge A^{\prime \prime}\right)\right)+\left(-2 A^{\prime \prime}+2 A_{n}^{\prime \prime} d x_{n}\right) & =0 \\
x_{n} d^{*}\left(A^{\prime \prime}\right)+A_{n}^{\prime \prime}-\left|A^{\prime \prime}\right|^{2} & =0
\end{aligned}
$$

where $A_{n}^{\prime \prime}$ is the $d x_{n}$ component of the 1 -form $A^{\prime \prime}$. After applying $d$ to the second equation and multiplying with $x_{n}$, we get the form:

$$
x_{n}^{2} d d^{*}\left(A^{\prime \prime}\right)+x_{n}\left(d^{*} A^{\prime \prime} \wedge d x_{n}+d\left(A_{n}^{\prime \prime}\right)-d\left(\left|A^{\prime \prime}\right|^{2}\right)\right)=0
$$

Now after adding the equation (8.7) to the equation (8.9) we get a degenerate elliptic second order non-linear equation, which has a diagonal principal part $x_{n}^{2} \Delta$ and every first order term multiplied with $x_{n}$. Notice also $A^{\prime \prime}=B^{\prime \prime}$ for $x_{n}>0$, so $A^{\prime \prime}-B^{\prime \prime}=O\left(x_{n}^{\infty}\right)$ on the hyperplane $x_{n}=0$.

By Corollary (11) in [53, we deduce that there exists a unique continuation principle for such equations and hence we obtain $A^{\prime \prime} \equiv B^{\prime \prime}$ in the lower space, by continuing from the hyperplane. More precisely, we may rewrite these non-linear equations for $A^{\prime \prime}$ and $B^{\prime \prime}$ in the form

$$
x_{n}^{2} \Delta A^{\prime \prime}=w\left(x, A^{\prime \prime}, \nabla A^{\prime \prime}\right) \quad \text { and } \quad x_{n}^{2} \Delta B^{\prime \prime}=w\left(x, B^{\prime \prime}, \nabla B^{\prime \prime}\right)
$$

where $w$ is a smooth function in its entries. Therefore, after subtracting these two and writing $C^{\prime \prime}=B^{\prime \prime}-A^{\prime \prime}$, we may obtain

$$
\begin{aligned}
x_{n}^{2} \Delta C^{\prime \prime}=w\left(x, B^{\prime \prime}, \nabla B^{\prime \prime}\right) & -w\left(x, A^{\prime \prime}, \nabla A^{\prime \prime}\right) \\
= & h_{1}\left(x, A^{\prime \prime}, B^{\prime \prime}, \nabla A^{\prime \prime}, \nabla B^{\prime \prime}\right) C^{\prime \prime}+h_{2}\left(x, A^{\prime \prime}, B^{\prime \prime}, \nabla A^{\prime \prime}, \nabla B^{\prime \prime}\right) \nabla C^{\prime \prime}
\end{aligned}
$$

by Taylor expanding the $w$ with respect to $C^{\prime \prime}$; here $h_{1}$ and $h_{2}$ are smooth in their entries and act linearly on $C^{\prime \prime}$ and $\nabla C^{\prime \prime}$, respectively. Thus, after fixing $h_{1}$ and $h_{2}$ as known functions, we may think of (8.10) as a linear system of equations (of real dimension $2 n$ ) in $C^{\prime \prime}$ and thus results from 53 apply.

Moreover, we have that $h=\frac{f_{1}}{g_{1}}$ carries smoothly over the hyperplane and therefore we have $d h=(B-A) h$ by subtracting equations 8.5 and (8.6), on the other side of the hyperplane. Furthermore, using the relation $d\left(|h|^{2}\right)=0$ obtained from the gauge equation, we immediately get that $|h|$ is constant and thus, non-zero so we may write $B=h^{*}(A)$.

Finally, by using Lemma 8.4 we deduce that $B=h^{*}(A)$ on the whole connected component (call it $\left.R^{\prime}\right)$ in $M \backslash \mathcal{N}(g)$ of the points in the lower space in the previously considered chart and therefore, that $h$ is non-zero on $R^{\prime}$ and that the boundary of $R^{\prime}$ are the points where (could be empty) $g=0$. This ends the procedure.

Observe that we may perform this procedure at the boundary for a dense set of points $p \in Q=\Gamma \cap \mathcal{N}(g)$ to extend $h$ such that $h^{*}(A)=B$ near these points with $h=1$ on the boundary. In more detail, the set $\{p \in Q \mid d g(p)=0$ or $d f(p)=0\}$ is small, in the sense that its complement is dense, by Lemma 8.4. On this set, near a point $p$, we may use semi-geodesic coordinates and write $f=x_{n} f_{1}$ and $g=x_{n} g_{1}$ as before; then $h=\frac{f_{1}}{g_{1}}$ extends $h$ smoothly and 
$h=1$ on boundary, since the DN maps agree. The boundary determination result applied to quantities $A^{\prime \prime}$ and $B^{\prime \prime}$ defined in (8.5) and (8.6) and the degenerate unique continuation result of Mazzeo now applies to equations (8.7) and (8.9), to uniquely extend from $\partial M$, as before.

We may now drill the holes and extend $h$ together with the relation $h^{*}(A)=B$, starting from the component of $V$, where we may use boundary determination. The idea is that drilling the holes connects path components over the possibly disconnecting set $\mathcal{N}(g)$. Let us now give an argument that what we are left with (after drilling the holes) is path connected.

Let us denote the complement of the zero set $T=M \backslash \mathcal{N}(g)$; obviously $M \backslash\left(\cup M_{i}\right) \subset T$ and $T$ open. Let $x_{0} \in M^{\circ}$ be a point in the open neighbourhood of $V$ where $B=h^{*}(A)$ and $y$ be any point in $T$. Consider any path $\gamma:[0,1] \rightarrow M$ with $\gamma(0)=x_{0}$ and $\gamma(1)=y$. We will construct a path $\gamma^{\prime}$ from $x_{0}$ to $y$, lying in $T$, by slightly perturbing the path $\gamma$, such that $\gamma$ and $\gamma^{\prime}$ are arbitrarily close. Let $d$ be the usual complete metric in the space $C^{\infty}([0,1], M)$ of smooth paths with fixed endpoints $x_{0}$ and $y$ (see Remark A.6 in the appendix).

By standard differential topology (see [38]), there exists an arbitrarily close path $\gamma_{1}$ to $\gamma$ (with the same endpoints), such that $\gamma_{1}$ intersects $M_{1}$ transversally in a finite number of points $P_{1}, \ldots, P_{k}$. There are two possibilities for these points, starting e.g. with $P=P_{1}$ :

(1) There exists a sequence of points $p_{i} \in M_{1}$, for $i=1,2, \ldots$, converging to $P$, such that $g\left(p_{i}\right) \neq 0$ for all $i$.

(2) We have $g=0$ in a neighbourhood of $P$ in $M_{1}$ and a sequence of points $q_{i} \in M_{1}$ converging to $P$, such that $d g\left(q_{i}\right) \neq 0$.

In the first case, we may slightly perturb $\gamma_{1}$, such that it goes through one of the points $p_{i}$ and is sufficiently close in the metric $d$. These are complementary conditions, so if the first item does not hold, then the second one does: in that case, we may still perturb $\gamma_{1}$ to go through one of the points $q_{i}$, by the above argument of drilling holes. Notice that each of the points $p_{i}$ or $q_{i}$ has a neighbourhood in $M_{1}$ through which we can perturb the curve and therefore, there exists an $\epsilon>0$, such that if we move our curve by a distance less than $\epsilon$ in the $d$-metric, the resulting curve will go through this neighbourhood.

Now inductively, we may perform the same procedure for all $j=1,2, \ldots, k$ and, each time, taking the perturbations small enough such that it does not interfere with the previously done work - what we obtain is $\gamma_{1}^{\prime}$, which is sufficiently close to $\gamma_{1}$ and which does not hit $M_{1}$, minus the deleted holes. Thus we obtain a Cauchy sequence of curves $\gamma_{1}^{\prime}, \gamma_{2}^{\prime}, \ldots$ such that $\gamma_{i}^{\prime}$ does not hit $M_{1}, M_{2}, \ldots, M_{i}$, minus the deleted holes. Since the space of curves is complete, we obtain a limiting curve $\gamma_{i}^{\prime} \rightarrow \gamma^{\prime}$, which lies completely in $T$ together with the drilled holes and furthermore satisfies $d\left(\gamma, \gamma^{\prime}\right)<\delta$ for some pre-fixed $\delta>0$. In particular, this implies that the lengths of the curves are close, i.e. $\left|l(\gamma)-l\left(\gamma^{\prime}\right)\right|<\delta^{\prime}$ for some $\delta^{\prime}>0$ (here $l$ denotes the length of the curve in the underlying Riemannian manifold). Let us denote the union of all of the drilled holes, i.e. neighbourhoods of some of the points $q_{i}$ in the item (2) above, by $T_{\gamma}$.

Moreover, we may repeat the above argument for all paths $\gamma$, now between any two points in $T$ - denote the set of new drilled holes by $S_{\gamma}$. Then we redefine $T$ as:

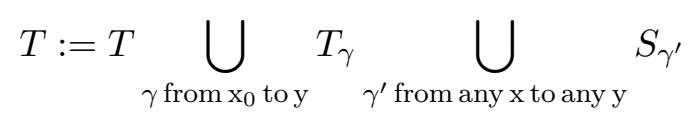

where the first union runs over all of the curves $\gamma$ starting at $x_{0}$ and ending at $y \in M^{\circ} \backslash \mathcal{N}(g)$; the second one is over all paths $\gamma^{\prime}$ between points in $M^{\circ} \backslash \mathcal{N}(g)$. It is easy to see that $T \subset M^{\circ}$ is open and connected and furthermore, it satisfies the property that for any curve $\gamma$ between any two points $x, y \in T$, there exists a sequence of curves $\gamma_{n}$ between $x$ and $y$, lying wholly in 
$T$, such that $d\left(\gamma_{n}, \gamma\right) \rightarrow 0$ as $n \rightarrow \infty$. Also, we have $B=h^{*}(A)$ on $T$ by the argument of drilling holes.

Let us denote by $d_{1}$ the inherited metric of $T$ as a subspace of $M$ and by $d_{2}$ the metric in the Riemannian manifold $\left(T,\left.g\right|_{T}\right)$. Therefore, as a result of the above construction, we may claim the following about these metrics ${ }^{2}$

$$
d_{2}(x, y)=\inf \{l(\gamma) \mid \gamma \text { a piecewise smooth path from } x \text { to } y \text { lying in } T\}=d_{1}(x, y)
$$

Notice also that we have, by the Fundamental Theorem of Calculus, if $\gamma$ is a path from $x$ to $y$ lying in $T$ :

$$
\begin{aligned}
|h(x)-h(y)|=\left|\int_{0}^{1} d h_{\gamma(t)}(\dot{\gamma}(t)) d t\right| \leq & \int_{0}^{1}\left|\left\langle\nabla h_{\gamma(t)}, \dot{\gamma}(t)\right\rangle\right| d t \\
& \leq \int_{0}^{1}\left|\nabla h_{\gamma(t)}\right|_{g} \cdot|\dot{\gamma}(t)|_{g} d t \leq C \int_{0}^{1}|\dot{\gamma}(t)|_{g} d t=C \cdot l(\gamma)
\end{aligned}
$$

by Cauchy-Schwarz, where $\nabla h$ is the gradient of $h$ and $C$ is a uniform bound on $d h$ (which follows from the global relation $d h=(B-A) h$ in $T$ and uniform bounds on $h, A$ and $B)$. If we take the infimum over all such curves $\gamma$, we obtain the inequality $|h(x)-h(y)| \leq C d_{2}(x, y)=C d_{1}(x, y)$ and therefore obtain that $h$ is Lipschitz and so uniformly continuous over $T$.

Therefore, $h$ can be extended continuously ${ }^{3}$ to the whole of $M$ and by inductively differentiating the relation $d h=(B-A) h$, we moreover have that all partial derivatives of $h$ can be continuously extended. That these continuous extensions of derivatives are actual derivatives of the extension of $h$ is proved in Lemma A.7 in the Appendix; see also Remark A.8 in the Appendix for the extension to the boundary. This proves $h^{*}(A)=B$ on the whole of $M$ with $h$ smooth and that $\left.h\right|_{\Gamma}=1 ; h$ also unitary. This finishes the proof.

REMARK 8.5 (Topological remarks). One can see that the complement of the disconnecting set $\mathcal{N}(g)$ can indeed have non-trivial topology; this justifies the use of our argument of drilling holes. For simplicity, we will consider real harmonic functions $g$ with $\Delta g=0$ in the open unit disk. Firstly, one may observe that there are two types of points in $\mathcal{N}(g)$ : the critical and the non-critical ones. The non-critical ones are simple: they are locally contained in an analytic curve, whereas the critical ones are isolated (since they are exactly the set of points where $f^{\prime}=0$, where $f$ holomorphic and $u=\operatorname{Re}(f))$ and are locally zero sets of harmonic polynomials, i.e. zero sets of $\operatorname{Re}\left((z-P)^{m}\right)$, where $m \geq 2$ an integer. Thus at these critical points, $\mathcal{N}(g)$ is a union of $m$ analytic curves meeting at $P$ at equal angles. Also, there are no loops in $\mathcal{N}(g)$, due to the uniqueness of the Dirichlet problem and analytic continuation. Therefore, if $g$ has an analytic extension to the closed disk, there are finitely many components in the complement of $\mathcal{N}(g)$, but if no such extension exists and $g$ is zero at infinitely many points at the boundary, then we may expect infinitely many components. This is because for each such vanishing, non-critical point of $g$ at the boundary we have an "end" coming inside the disk, which returns to the boundary at some other point, by the analysis above. See Figure 1 for a concrete example and $\mathbf{1 6}$, 8 for further analysis.

REMARK 8.6. If given $d_{A}^{*} d_{A} F=0$ we were able to write down an elliptic equation for $\operatorname{det}(F)$, then all (or almost all) of the above proof would carry over to the case of vector bundles

\footnotetext{
${ }^{2}$ We just proved that the inherited subspace metric on $T \subset M$ and the path metric as a submanifold of a Riemannian manifold are Lipschitz equivalent with Lipschitz constant equal to 1.

${ }^{3}$ Here we are using the elementary fact that a uniformly continuous function can be uniquely continuously extended to the closure of its domain.
} 


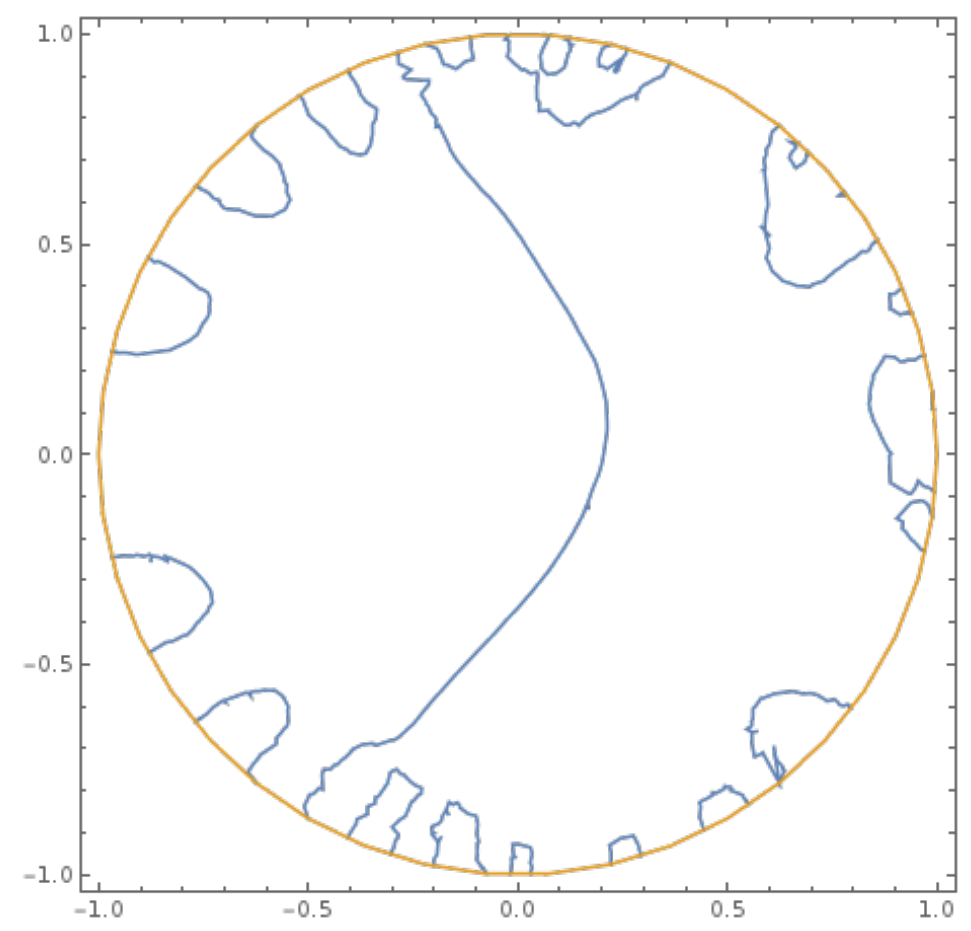

FiguRE 1. In blue - the zero set of the harmonic function with boundary value equal to $f(\theta)=\theta \cdot \sin \frac{100}{\theta}$ on the unit disc, where $\theta \in(-\pi, \pi)$ is the angular coordinate. In orange - the unit circle. The accumulation point is $(1,0)$.

of higher rank, as in Theorem 8.2 . Also, notice that the only two implications we were using in Theorem $\mathrm{G}$ from the equality of the DN maps for $A$ and $B$, were:

(1) By boundary determination, the connections $A$ and $B$ have the same full jets at the boundary in suitable gauges.

(2) There exist two non-zero solutions $f$ and $g$ to $d_{A}^{*} d_{A} f=d_{B}^{*} d_{B} g=0$, such that $\left.f\right|_{\partial M}=$ $\left.g\right|_{\partial M}$ and $\left.\partial_{\nu} f\right|_{\Gamma}=\left.\partial_{\nu} g\right|_{\Gamma}$ for a non-empty open $\Gamma \subset \partial M$.

REMARK 8.7 (Alternative boundary extension). A different approach to extension of the gauge to the boundary, by using the partial differential equations that it satisfies (that is $H^{-1} d H+H^{-1} A H=B$ ), can be found in Proposition 4.7 from [44. There, the authors take $A$ and $B$ to a gauge with no normal component (as in Lemma 2.4), so that the new gauge $H^{\prime}$ is independent of the normal variable from the equation it satisfies and can clearly be extended smoothly. Note that the same proof works in the non-unitary case.

By a careful analysis of the proof of Theorem G, we have the following result:

Proposition 8.8. Conclusions of Theorem $G$ hold in the non-unitary case.

Proof. We use the same notation as in the original proof. The first issue is that we do not know that $d\left(|h|^{2}\right)=0$ any more, so a priori $f$ and $g$ need not have the same zero set. We address this as follows.

By gauge transforming $A$ and $B$ locally near a zero set hypersurface $g^{-1}(0)$ of $g$ (or $f^{-1}(0)$ for $f$ ), we may assume that the $d x_{n}$ components of $A$ and $B$ vanish and $B=h^{*}(A)$ in $\left\{x_{n}>0\right\}$. Then by Remark 8.7, we see that $h$ is independent of $x_{n}$ and so extends in a non-singular way beyond $\left\{x_{n}=0\right\}$ - thus $\frac{f}{g}$ also extends in a smooth and non-zero way. From that point, we may apply the earlier argument in the same way. 
By drilling along paths as before, we are left with $h: T \rightarrow \mathbb{C}$ such that $h^{*}(A)=B(h$ is nowhere zero) and $\left.h\right|_{\Gamma}=1$, where $T$ is dense, connected and open and moreover, $T$ satisfies the property that given a curve $\gamma$ in $M$ with endpoints in $T$, there exist arbitrarily close curves to $\gamma$ with the same endpoints, lying wholly in $T$. Notice that $d h=(B-A) h$ on $T$ implies $d A=d B$ by density, which by Remark 7.7 immediately proves the claim. Alternatively, we will extend $h$ to a gauge on $M^{\circ}$ by proving uniform bounds on $h$ on compact subsets of $M^{\circ}$.

Take a point $p \in M^{\circ} \backslash T$. Note that we have in $T$

$$
d h=(B-A) h
$$

So if we take a small ball $U$ around $p$, we have a logarithm $f$ in $U$, by solving $d f=B-A$ (such an $f$ exists as $d A=d B$ ). Then by uniqueness we have $h=c \cdot e^{f}$ for a constant $c$, as $U \cap T$ is connected. So $h$ extends smoothly on $U$ and by density, we have $h^{*}(A)=B$ on $U$. So $h$ extends to $M^{\circ}$, such that $h^{*}(A)=B$. We are left to observe that Remark 8.7 extends $h$ smoothly to $\partial M$.

\subsection{Recovering a Yang-Mills connection for $m>1$}

The main obstacle to solving the $m>1$ case is the possibility that the zero set of $\operatorname{det} F$ for $F$ satisfying $d_{A}^{*} d_{A} F=0$ could potentially be large; it suffices to prove that the determinant does not vanish to an infinite order (if non-trivial) at any point, since by some general theory the zero set is then contained in an $(n-1)$-rectifiable set $\mathbf{7}$. In other words, we want to prove $\operatorname{det} F$ satisfies the strong unique continuation property. In addition to this, we would like to point out that it is no longer true that the critical zero set of $\operatorname{det} F$ is $(n-2)$-rectifiable, as in the case $m=1$; a class of counterexamples is given by $F=\left(\begin{array}{ll}f & 0 \\ 0 & f\end{array}\right)$, where we have that $\mathcal{N}_{\text {crit }}(\operatorname{det} F)=\mathcal{N}_{\text {crit }}\left(f^{2}\right)$ contains the set where $f$ vanishes (since $d\left(f^{2}\right)=2 f d f$ ). Therefore if $f$ vanishes on an $(n-1)$-dimensional set, then the critical set is also $(n-1)$-dimensional. One such example is given by $M=\mathbb{R}^{2}$ and $f(x, y)=x$ which vanishes along the $y$-axis and solves $\Delta_{\mathbb{R}^{2}}(x)=0$.

Here a function $f$ on a manifold $M$ vanishes to infinite order at a point $x_{0} \in M$ if in any local

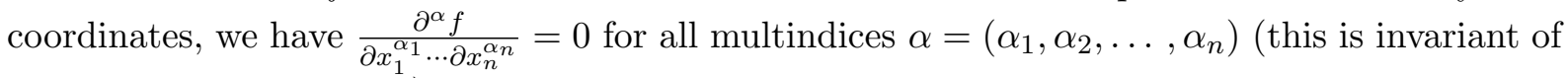
the choice of coordinates).

However, if we were able to prove that $\operatorname{det} F$ has the strong unique continuation property, then we may try and run through the argument in Theorem 7.7 again. We will therefore consider the analytic case first - analytic functions satisfy the SUCP by definition and in addition, the zero set is given by a countable union of analytic submanifolds of codimension one. This can easily be seen by considering the order of vanishing at a point and by observing that locally, every point in the zero set is contained in $\left(\partial^{\alpha} g\right)^{-1}(0)$, where $g$ is the analytic function and $\alpha$ is a multi-index such that $\nabla\left(\partial^{\alpha} g\right) \neq 0$.

Note that if $A$ and $g$ are analytic, one has $F$ satisfying $d_{A}^{*} d_{A} F=0$, which is an elliptic system with analytic coefficients and thus by a classical result of Morrey [56 its entries are analytic. Therefore, the determinant is analytic also and thus cannot vanish to the infinite order at a single point, if it is non-trivial. Unless otherwise stated, for the rest of the section $(M, g)$ is a compact analytic (in the interior) Riemannian manifold of dimension $n$ with boundary. We first prove a result about the zero set of the determinant of a matrix solution where $A$ is Yang-Mills and not necessarily analytic: 
Lemma 8.9. Let $E=M \times \mathbb{C}^{m}$ a Hermitian vector bundle and $A$ a unitary Yang-Mills connection on $E$. Then any solution $F: M \rightarrow \mathbb{C}^{m \times m}$ to $d_{A}^{*} d_{A} F=0$ with $\operatorname{det} F$ non-zero has $\mathcal{N}(\operatorname{det} F)$ to be $(n-1)$-rectifiable. Moreover, $\operatorname{det} F$ satisfies the strong unique continuation property.

Proof. This is a local result, so assume we have a point $p \in M^{\circ}$ in the interior and take a small coordinate ball $B^{n}(\epsilon)$ around $p$, such that $\|A\|_{L^{n / 2}\left(B^{n}(\epsilon)\right)}$ is small enough; by a dilation we may also assume $B^{n}$ is the unit ball and we also have $\|A\|_{L^{n / 2}\left(B^{n}\right)}$ stays the same as for the smaller ball, by a computation. By a result of Uhlenbeck $\mathbf{8 3}$, we have a gauge automorphism $X: B^{n} \rightarrow U(m)$ that takes $A$ to $A^{\prime}=X^{*}(A)$ with $d^{*}\left(A^{\prime}\right)=0$. In this particular gauge, the Yang-Mills equations become elliptic and therefore, $A^{\prime}$ is analytic.

Similarly, since $d_{A}^{*} d_{A} F=0$, we have $d_{A^{\prime}}^{*} d_{A^{\prime}} F^{\prime}=0$, where $F^{\prime}=X^{-1} F$ - thus $F^{\prime}$ is also analytic. Moreover, $\operatorname{det} F^{\prime} \operatorname{det} X=\operatorname{det} F$ and so $\mathcal{N}(\operatorname{det} F)=\mathcal{N}\left(\operatorname{det} F^{\prime}\right)$ on $B^{n}$, as $X$ is always invertible; since $\operatorname{det} F^{\prime}$ is analytic, we obtain the first part of the result.

Finally, from the relation $\operatorname{det} F^{\prime} \operatorname{det} X=\operatorname{det} F$ and the fact that $\operatorname{det} X$ is non-zero on $B^{n}$, we immediately get that $\operatorname{det} F$ vanishes up to order $k$ if and only if $\operatorname{det} F^{\prime}$ vanishes up to order $k$ - thus $\operatorname{det} F$ satisfies the SUCP, as $\operatorname{det} F^{\prime}$ does.

This means that on $M^{\circ}$ we have $\mathcal{N}(\operatorname{det} G) \subset \cup_{i} M_{i}$ for $M_{i}$ a countable family of analytic submanifolds of codimension one, where $G$ solves $d_{B}^{*} d_{B} G=0$ and represents the gauge we used in the previous section. Notice that $G^{*}(B)$ then satisfies an elliptic system (as before), but with analytic coefficients - therefore $G^{*}(B)$ is also analytic, but only on the set where $G$ is invertible.

To overcome this, we use the method of proof of the $m=1$ case, Theorem $\mathrm{G}$, and the main difference is that now we will be able to use analyticity to uniquely continue the solution when drilling hyperplanes, whereas before we relied on the unique continuation property of elliptic equations.

Proof of Theorem [H. Assume we have the gauges $F$ and $G$ that solve $d_{A}^{*} d_{A} F=0$ and $d_{B}^{*} d_{B} G=0$ with $\left.F\right|_{\partial M}=\left.G\right|_{\partial M}, \operatorname{supp}\left(\left.F\right|_{\partial M}\right)=\operatorname{supp}\left(\left.G\right|_{\partial M}\right) \subset \Gamma$ and equal to identity on an open, non-empty subset $V$ of $\Gamma$. Then $F^{*}(A)=G^{*}(B)$ in the neighbourhood $U$ of $V$ in the manifold, as in Theorem 8.2 by unique continuation; equivalently, we have $H^{*}(A)=B$ where $H=F G^{-1}$. We also have that $H$ is unitary.

We may suppose that $\mathcal{N}(\operatorname{det} G) \subset \cup_{i} M_{i}$ for $M_{i}$ analytic submanifolds of codimension one, by Lemma 8.9. Let us now prepare the terrain for drilling the holes - consider a point $p$ in $M_{i}$ for some $i$ and assume $\operatorname{det} G=0$ near $p$ in $M_{i}$, such that the following property holds:

$$
\frac{\partial^{j}(\operatorname{det} G)}{\partial x_{n}^{j}}=0 \quad \text { for } \quad j=0,1, \ldots, k-1
$$

in a neighbourhood of $p$ in $M_{i}$, for some $k$; we also ask that $\frac{\partial^{k}(\operatorname{det} G)}{\partial x_{n}^{k}}(p) \neq 0$. Here we are using the analytic chart given by coordinates on $M_{i}$ near $p$ and the $x_{n}$ coordinate given by following the normal geodesics (which is also analytic). We make the standing assumption that $F$ and $G$ are invertible for $x_{n}>0$ in this coordinate system and that $F^{*}(A)=G^{*}(B)$ in the same set.

This means that near $p$, by Taylor's theorem we have $\operatorname{det} G=x_{n}^{k} g_{1}$ for some $g_{1}$ that satisfies $g_{1}(p) \neq 0$ - therefore locally at $p, \mathcal{N}(\operatorname{det} G)$ is contained in $M_{i}$. Since $H$ is unitary for $x_{n}>0$, we have $H=F G^{-1}=\frac{F \operatorname{adj} G}{x_{n}^{k} g_{1}}$ is bounded on this set and therefore $F \operatorname{adj} G=x_{n}^{k} H_{1}$ for some smooth $H_{1}$ near $p$ - we get $H=\frac{H_{1}}{g_{1}}$ locally, which means that $H$ extends smoothly to the other side of $M_{i}$ in the proximity of $p$. Moreover, as $H$ unitary we have $|\operatorname{det} H|=1$ at $p$ and so $H$ is invertible near $p$. 
To use the real-analyticity, we must transform $A$ and $B$ such that they are locally analytic we do this by constructing the Coulomb gauge automorphisms (unitary) $X$ and $Y$ locally near $p$ such that $A^{\prime}=X^{*}(A)$ and $B^{\prime}=Y^{*}(B)$ and moreover, that $d^{*}\left(A^{\prime}\right)=d^{*}\left(B^{\prime}\right)=0$ (by the proof of Lemma 8.9). Then $A^{\prime}$ and $B^{\prime}$ are analytic as in the previous lemma and moreover, we have $F^{\prime}=X^{-1} F$ and $G^{\prime}=Y^{-1} G$ satisfying $d_{A^{\prime}}^{*} d_{A^{\prime}} F^{\prime}=0$ and $d_{B^{\prime}}^{*} d_{B^{\prime}} G^{\prime}=0$ - therefore $F^{\prime}$ and $G^{\prime}$ are analytic, as well.

Thus we may write $H^{\prime}=X^{-1} F G^{-1} Y$ and by rewriting $H^{*}(A)=B$ (by assumption) we get $H^{\prime *}\left(A^{\prime}\right)=B^{\prime}$ for $x_{n}>0$ in a neighbourhood of $p$. Let us now observe that $H^{\prime}$ also smoothly (analytically) extends over the hyperplane $M_{i}$ - this is because, by Taylor expanding $\operatorname{det}\left(Y^{-1} G\right)=\frac{\operatorname{det} G}{\operatorname{det} Y}$, we get

$$
H^{\prime}=X^{-1} F \cdot \frac{\operatorname{adj}\left(Y^{-1} G\right)}{g^{\prime} x_{n}^{k}}
$$

where $g^{\prime}=\frac{g_{1}}{\operatorname{det} Y}$ is analytic, so $g^{\prime} \neq 0$ near $p$. However, we know $H^{\prime}$ is bounded near $p$, since $H$, $X$ and $Y$ are. Thus $X^{-1} F \cdot \operatorname{adj}\left(Y^{-1} G\right)=F^{\prime} \cdot \operatorname{adj} G^{\prime}=x_{n}^{k} H^{\prime \prime}$ for some analytic $H^{\prime \prime}$, by looking at the expansion of $F^{\prime}$ adj $\left(G^{\prime}\right)$ - in conclusion, $H^{\prime}=\frac{H^{\prime \prime}}{g^{\prime}}$ analytically extends near $p$ and $H^{\prime}$ is also invertible at $p$ as $H, X$ and $Y$ are.

Finally, it is easy now to see that $\left(H^{\prime}\right)^{*}\left(A^{\prime}\right) \equiv B^{\prime}$, since both sides are analytic near $p$ and $\left(H^{\prime}\right)^{*}\left(A^{\prime}\right)=B^{\prime}$ for $x_{n}>0$; equivalently $H^{*}(A) \equiv B$ near $p$. This ends the drilling argument and we may repeat the part of the argument of Theorem $\mathrm{G}$ which perturbs the curve by an arbitrarily small amount so that it goes through the holes.

Let us briefly describe the analogous procedure from Theorem G. Take a base point $x_{0} \in$ $U \cap M^{\circ}$ and consider a path $\gamma$ lying in the interior, from $x_{0}$ to some point $y \in M^{\circ}$. We perturb $\gamma$ such that it intersects $M_{1}$ transversally at $P_{1}, \cdots P_{k}$ ( $k$ can be zero). At $P_{1}$, consider the tubular neighbourhood (analytic) given by following geodesics perpendicular to $M_{1}$. If there exists a sequence of points $p_{j} \in M_{1}$ that converges to $P_{1}$ and $\operatorname{det} G \neq 0$ at every $p_{j}$, we may perturb $\gamma$ slightly and get it to pass through one of the points $p_{j}$. Otherwise, inductively, since $\operatorname{det} G$ satisfies the SUCP by Lemma 8.9 there exists a positive integer $k$ such that $\frac{\partial^{i}(\operatorname{det} G)}{\partial x_{n}^{i}}=0$ for $0 \leq i \leq k-1$ in a neighbourhood of $P_{1}$ and there exists a sequence of points $p_{j} \in M_{1}$ that converge to $P_{1}$ and $\frac{\partial^{k}(\operatorname{det} G)}{\partial x_{n}^{k}} \neq 0$ at each $p_{j}$. This leaves us in the setting (8.11) from the previous paragraph, suitable for drilling the holes - inductively, we perturb $\gamma$ such that it intersects the $M_{i}$ in the drilled holes.

Thus we obtain a smooth (analytic in the interior) extension of $H=F G^{-1}$ to the whole of $M$, such that $H^{*}(A)=B$ and $\left.H\right|_{V}=I d$.

To get the wanted gauge with $\left.H\right|_{\Gamma}=I d$, we will need a slightly different argument, because we do not know if $\operatorname{det} F$ and $\operatorname{det} G$ vanish to infinite order at the boundary, as we did not assume analyticity up to the boundary. We will construct a sequence of matrix functions $H_{i}$ such that $H_{i}^{*}(A)=B$ and use a compactness argument to take the limit. Consider nested open sets $V_{i}$, with $\emptyset \neq V_{1} \varsubsetneqq \bar{V}_{1} \varsubsetneqq V_{2} \varsubsetneqq \bar{V}_{2} \varsubsetneqq \ldots \varsubsetneqq \Gamma$ with the property $\cup_{i} V_{i}=\Gamma$. Construct appropriate $F_{i}$ and $G_{i}$ supported in $\Gamma$, such that $\left.F_{i}\right|_{V_{i}}=\left.G_{i}\right|_{V_{i}}=I d$, solving $d_{A}^{*} d_{A} F_{i}=0$ and $d_{B}^{*} d_{B} G_{i}=0$ and setting $H_{i}=F_{i} G_{i}^{-1}$ - by the argument above $H_{i}^{*}(A)=B$ and $\left.H_{i}\right|_{V_{i}}=I d$. Now the important property that the gauges satisfy is that they are unitary, hence bounded and they satisfy $d H_{i}=H_{i} B-A H_{i}$ so that $d H_{i}$ are uniformly bounded. By inductively differentiating this relation, we get that all derivatives of $H_{i}$ are uniformly bounded on $M$. By the Arzelà-Ascoli theorem (or the Heine-Borel property of $C^{\infty}(M)$ ) we get a convergent subsequence with a limit $H \in C^{\infty}(M ; U(m)),\left.H\right|_{\Gamma}=I d$ and $H^{*}(A)=B$. This finishes the proof. 
REMARK 8.10. If we were able to prove that the $\operatorname{determinant} \operatorname{det} F$ satisfies the strong unique continuation property (where $d_{A}^{*} d_{A} F=0$ ) and that the unique continuation property from a hyperplane holds for degenerate elliptic systems, with degeneracies of the form $x_{n}^{2 k} \Delta_{g} \times$ $I d+x_{n}^{k} F_{1}+F_{0}$, where $F_{0}$ and $F_{1}$ are zero and first order matrix operators, respectively and for all $k$ positive integers - then we would be able to solve the $m>1$ case in the smooth category, by following the proofs of Theorems $\mathrm{G}$ and $\mathrm{H}$.

REMARK 8.11. As mentioned, while $A^{\prime}=F^{*}(A)$ above satisfies an elliptic equation with analytic coefficients and hence is analytic (and so is $F$ ), a problem appears when we approach the singular set of $F$. Then we do not know any more that $A^{\prime}$ is analytic and cannot apply analyticity directly to conclude $F^{*}(A) \equiv G^{*}(B)$. However, as we have seen in the above proof we may use the Coulomb gauge locally to get around this issue. 


\section{APPENDIX A}

\section{Some less obvious elementary facts}

Here we prove some of the less obvious elementary facts that we use in the thesis.

\section{A.1. The DN map}

We prove that $\Lambda_{A, Q}: H^{\frac{1}{2}}\left(\partial M ;\left.E\right|_{\partial M}\right) \rightarrow H^{-\frac{1}{2}}\left(\partial M ;\left.E\right|_{\partial M}\right)$ is a bounded linear map and that $H^{\frac{1}{2}}\left(\partial M ;\left.E\right|_{\partial M}\right)$, defined in the standard way, is isomorphic to the quotient $H^{1}(M ; E) / H_{0}^{1}(M ; E)$, so that Definition 2.3 makes sense. For brevity, we drop the manifold and bundle symbols from the Sobolev spaces notation.

Proposition A.1. Definition 2.1 makes sense, i.e. $\Lambda_{A, Q}$ is well-defined and bounded.

Proof. We need to check that the definition is independent of the choice of $e_{h}$ : let $\varphi \in$ $H_{0}^{1}(M ; E)$. We need to show that

$$
\int_{M}\left[\left\langle d_{A} u_{f}, d_{A} \varphi\right\rangle+\left\langle Q u_{f}, \varphi\right\rangle\right] d V=0
$$

but that follows directly from the fact that $u_{f}$ is a weak solution of 2.2. Moreover, $u_{f}$ is clearly well-defined for $f \in H^{\frac{1}{2}}$ and the map is linear in both $f$ and $h$.

Next we check the range lies in $H^{-\frac{1}{2}}$.

$$
\left|\left\langle\left\langle\Lambda_{A, Q} f, h\right\rangle\right\rangle\right| \leq\left\|u_{f}\right\|_{H^{1}}\left\|e_{h}\right\|_{H^{1}}+\|Q\|_{L^{\infty}}\left\|u_{f}\right\|_{L^{2}}\left\|e_{h}\right\|_{L^{2}} \leq C\left\|u_{f}\right\|_{H^{1}}\left\|e_{h}\right\|_{H^{1}}
$$

for a constant $C$. Since $e_{h}$ was arbitrary representative of the class of $h$, we conclude

$$
\left|\left\langle\left\langle\Lambda_{A, Q} f, h\right\rangle\right\rangle\right| \leq C\left\|u_{f}\right\|_{H^{1}}\|h\|_{H^{\frac{1}{2}}}
$$

so $\Lambda_{A, Q} f \in H^{-\frac{1}{2}}$. Furthermore, using the previous inequality, we have that:

$$
\left\|\Lambda_{A, Q} f\right\|_{H^{-\frac{1}{2}}} \leq C\left\|u_{f}\right\|_{H^{1}}
$$

Since $\mathcal{L}_{A, Q}: H_{0}^{1} \rightarrow H^{-1}$ is an isomorphism by assumption, we have constants $C_{1}$ and $C_{2}$ so that $C_{1}\|v\|_{H^{1}} \leq\left\|\mathcal{L}_{A, Q} v\right\|_{H^{-1}} \leq C_{2}\|v\|_{H^{1}}$ for all $v \in H_{0}^{1}$ and thus, for all $e_{f} \in H^{1}$ that represent the class of $f$ :

$$
\left\|u_{f}\right\|_{H^{1}} \leq\left\|u_{f}-e_{f}\right\|_{H^{1}}+\left\|e_{f}\right\|_{H^{1}} \leq \frac{1}{C_{1}}\left\|\mathcal{L}_{A, Q} e_{f}\right\|_{H^{-1}}+\left\|e_{f}\right\|_{H^{1}} \leq C^{\prime}\left\|e_{f}\right\|_{H^{1}}
$$

for some constant $C^{\prime}$, since $\mathcal{L}_{A, Q}: H^{1} \rightarrow H^{-1}$ is bounded. Since $e_{f}$ was arbitrary, we conclude that

which finishes the proof.

$$
\left\|u_{f}\right\|_{H^{1}} \leq C^{\prime}\|f\|_{H^{\frac{1}{2}}}
$$

Now let us recall the definitions of Sobolev spaces on manifolds for a real parameter. Firstly, the Sobolev spaces $H^{s}\left(\mathbb{R}^{n}\right)$ can be defined by completing the space of smooth compactly supported functions in the norm:

$$
\|u\|_{H^{s}\left(\mathbb{R}^{n}\right)}^{2}=\int_{\mathbb{R}^{n}}\left(1+|\xi|^{2}\right)^{s}|\hat{u}(\xi)|^{2} d \xi
$$


where $\hat{u}(\xi)=\int_{\mathbb{R}^{n}} e^{-i x \cdot \xi} u(x) d x$ is the Fourier transform of $u$. Observe that we may also define the norm of vector valued functions, by just taking the sum of the norms of the components; we denote the corresponding Hilbert space by $H^{s}\left(\mathbb{R}^{n} ; \mathbb{C}^{m}\right)$. Furthermore, for a smooth domain $\Omega \subset \mathbb{R}^{n}$ and $s \geq 0$, we may define the Sobolev spaces by taking completions:

$$
\begin{aligned}
& H_{0}^{s}(\Omega):=\overline{C_{0}^{\infty}(\Omega)}\|\cdot\|_{H^{s}(\Omega)} \quad \text { with norm }\|\cdot\|_{H^{s}(\Omega)} \\
& H^{s}(\Omega):=\left.H^{s}\left(\mathbb{R}^{n}\right)\right|_{\Omega} \quad \text { with norm } \quad\|u\|_{H^{s}(\Omega)}:=\inf \left\{\|\tilde{u}\|_{H^{s}\left(\mathbb{R}^{n}\right)}|\tilde{u}|_{\Omega}=u\right\}
\end{aligned}
$$

It can be shown that the second space is the closure of functions in $C^{\infty}(\bar{\Omega})$ with compact support in $\bar{\Omega}$ (by just using that $C_{0}^{\infty}\left(\mathbb{R}^{n}\right)$ is dense in $H^{s}\left(\mathbb{R}^{n}\right)$ ).

Now fix a smooth compact manifold $X$ without boundary and a vector bundle $E$ of rank $m$ over it and consider a family of charts $\left\{\left(U_{i}, \varphi_{i}\right)\right\}$ covering $M$, associated with the family of trivialisations of the bundle $\left\{\psi_{i}\right\}$ and with a partition of unity $\left\{\rho_{i}\right\}$ subordinate to $U_{i}$. By transferring the norms to the charts, we may define Sobolev spaces on $E$ over $X$ :

Definition A.2 (Sobolev spaces on manifolds). For a smooth section $f$ of $E$ over $X$, we may define the Sobolev norm as follows:

$$
\|f\|_{H^{s}(X ; E)}^{2}:=\sum_{i}\left\|\psi_{i}\left(\rho_{i} f\right) \circ \varphi_{i}^{-1}\right\|_{H^{s}\left(\varphi_{i}\left(U_{i}\right) ; \mathbb{C}^{m}\right)}^{2}
$$

The Sobolev space $H^{s}(X ; E)$ for $s \in \mathbb{R}$ is defined as the following completion:

$$
H^{s}(X ; E):=\overline{C^{\infty}(X ; E)} \|^{\|\cdot\|_{H^{s}(X ; E)}} \quad \text { with norm } \quad\|\cdot\|_{H^{s}(X ; E)}
$$

By using the invariance of Sobolev spaces under diffeomorphisms and changes of local frames, and the continuity of the multiplication map by a smooth compactly supported function, we may get that the just defined spaces are invariantly defined and that moreover, the norms defined by a different choice of charts and a partition of unity are isomorphic. Moreover, the intrinsic definition of $H^{1}$ from Chapter 2 agrees with the above one.

Let now $M$ be a compact manifold with boundary, with a vector bundle $E$ over $M$ and let $X$ be its double, i.e. $X=M \cup_{\partial M} M$ obtained by gluing the two collars near the boundary. Moreover, the structures vector bundles, metric, connections over $M$ are naturally extended to the double $X$; we will still write $E$ for the extension of the vector bundle to $X$. We may now define (see 8,54 for more details):

Definition A.3 (Sobolev spaces on manifolds with boundary). The Sobolev spaces associated to $X$ and $E$ are defined as, for $s \geq 0$, where the restriction is taken in the $L^{2}$ spaces:

$$
\begin{aligned}
& H^{s}(M ; E):=\left.H^{s}(X ; E)\right|_{M} \quad \text { with norm } \quad\|u\|_{H^{s}(M ; E)}:=\inf \left\{\|\tilde{u}\|_{H^{s}(X)}|\tilde{u}|_{M}=u\right\} \\
& H_{0}^{s}(M ; E):=\bar{C}_{0}^{\infty}(M ; E)^{\|\cdot\|_{H^{s}(X ; E)}} \quad \text { with norm } \quad\|\cdot\|_{H^{s}(X ; E)}
\end{aligned}
$$

It can be checked that we obtain the same spaces with equivalent norms, if we take any other extension of $M$ to a manifold without boundary. Moreover, there exists a continuous right inverse to the extension map - for every $k \in \mathbb{N}$, there exists an operator $E_{k}: H^{k}(M ; E) \rightarrow H^{k}(X ; E)$. Locally, if we consider the upper half-plane $\mathbb{H}^{n} \subset \mathbb{R}^{n}$ with the coordinates $(y, t)$, we may define $E_{k}^{\prime}$ for $u \in H^{k}\left(\mathbb{H}^{n}\right)$ to be:

$$
E_{k}^{\prime}(u)(y, t)=\sum_{j=1}^{k+1} a_{j} u(y,-j t) \quad \text { for } \quad t<0
$$


and $E_{k}^{\prime}(u)(y, t)=u(y, t)$ for $t>0$, where the $a_{j}$ solve the following linear system of equations (a Vandermonde's determinant is non-zero):

$$
\sum_{j=1}^{k+1}(-j)^{l} a_{j}=1 \quad \text { for } \quad l=0, \ldots, k
$$

Observe that if we take $k>s \geq 0$, then $E_{k}^{\prime}: H^{s}\left(\mathbb{H}^{n}\right) \rightarrow H^{s}\left(\mathbb{R}^{n}\right)$ is continuous and that our discussion generalises to the $\mathbb{C}^{m}$-valued functions case. Then if we cut off near the boundary and take a suitable partition of unity subordinate to a cover close to the boundary, we obtain a continuous extension map $E_{s}: H^{s}(M ; E) \rightarrow H^{s}(X ; E)$ by transferring the problem to local coordinates.

We are now ready to prove the following:

Proposition A.4 (Characterisation of $H^{\frac{1}{2}}$ ). There exists a unique, bounded trace map $T: H^{1}(M ; E) \rightarrow H^{\frac{1}{2}}\left(\partial M ;\left.E\right|_{\partial M}\right)^{1}$ such that $T u=\left.u\right|_{\partial M}$ for smooth $u$. Moreover, $T$ is surjective with kernel equal to $H_{0}^{1}(M){ }^{2}$

Proof. It suffices to prove that there is a trace map $T_{1}$, such that $T_{1}: H^{1}(X ; E) \rightarrow$ $H^{\frac{1}{2}}\left(\partial M ;\left.E\right|_{\partial M}\right)$ is continuous and takes a smooth function to its restriction; then $T:=T_{1} \circ E_{s}$ does the job.

Take a finite set of charts $\left\{\left(U_{i}, \varphi_{i}\right)\right\}$ for $i=1, \ldots, N_{0}$ with trivialisation $\left\{\psi_{i}\right\}$ for $E$ over $M$ that covers $\partial M$, such that $\varphi_{i}\left(U_{i}\right)=\mathbb{R}^{n}$ and denote the parts of the boundary by $\Gamma_{i}=U_{i} \cap \partial M$. Take a partition of unity $\rho_{i}$ subordinate to $\Gamma_{i}$ and extend it to a partition of unity $\rho_{j}$ for $j \geq 0$, where we added an open set $U_{0}$ to cover the whole of $X$. Then for $u \in C^{\infty}(X ; E)$ :

$$
\begin{aligned}
\|u\|_{H^{1}(X ; E)} & \gtrsim \sum_{i=1}^{N_{0}}\left\|\psi_{i}^{-1} \cdot\left(\rho_{i} u\right) \circ \varphi_{i}^{-1}\right\|_{H^{1}\left(\mathbb{R}^{n} ; \mathbb{C}^{m}\right)} \\
\|u\|_{H^{\frac{1}{2}}\left(\partial M ;\left.E\right|_{\partial M}\right)} & \lesssim \sum_{i=1}^{N_{0}}\left\|\psi_{i}^{-1} \cdot\left(\rho_{i} u\right) \circ \varphi^{-1}\right\|_{H^{\frac{1}{2}\left(\mathbb{R}^{n-1} ; \mathbb{C}^{m}\right)}}
\end{aligned}
$$

Therefore, to prove $T_{1}$ well-defined and continuous, it suffices to prove that $\left\|\left.v\right|_{\mathbb{R}^{n-1}}\right\|_{H^{\frac{1}{2}}\left(\mathbb{R}^{n-1}\right)} \lesssim$ $\|v\|_{H^{1}\left(\mathbb{R}^{n}\right)}$ for $v \in C_{0}^{\infty}\left(\mathbb{R}^{n}\right)$; we do this as follows.

Let us estimate the $H^{\frac{1}{2}}$ norm of $\left.v\right|_{\mathcal{R}^{n-1}}$ - if we write the first $n-1$ coordinates in $\mathbb{R}^{n-1}$ as $x^{\prime}$, by the Fourier inversion formula we have:

$$
v\left(x^{\prime}, 0\right)=T_{1}(v)\left(x^{\prime}\right)=\frac{1}{2 \pi} \int_{\mathbb{R}^{n}} e^{i x^{\prime} \cdot \xi^{\prime}} \hat{v}(\xi) d \xi=\frac{1}{2 \pi} \int_{\mathbb{R}^{n-1}}\left(\int_{\mathbb{R}} \hat{v}\left(\xi^{\prime}, \xi_{n}\right) d \xi_{n}\right) e^{i x^{\prime} \cdot \xi^{\prime}} d \xi^{\prime}
$$

Let $s=1$. For a function $f$ in $L^{2}\left(\mathbb{R}^{n-1}\right)$, define $\tilde{f}$ to be its Fourier transform. By the above and the inverse Fourier transform, we have:

$$
\widetilde{T v}\left(\xi^{\prime}\right)=\int_{\mathbb{R}} \hat{v}\left(\xi^{\prime}, \xi_{n}\right) d \xi_{n}=\int_{\mathbb{R}}\left(1+|\xi|^{2}\right)^{-\frac{s}{2}}\left(1+|\xi|^{2}\right)^{\frac{s}{2}} \hat{v}\left(\xi^{\prime}, \xi_{n}\right) d \xi_{n}
$$

and so by Cauchy-Schwarz:

$$
\left|\widetilde{T v}\left(\xi^{\prime}\right)\right|^{2} \leq \int_{\mathbb{R}}\left(1+|\xi|^{2}\right)^{-s} d \xi_{n} \int_{\mathbb{R}}\left(1+|\xi|^{2}\right)^{s}|\hat{v}|^{2} d \xi_{n}
$$

Use the substitution $\xi_{n}=t\left(1+|\xi|^{2}\right)$ to get:

$$
M_{s}\left(\xi^{\prime}\right)=\int_{\mathbb{R}}\left(1+|\xi|^{2}\right)^{-s} d \xi_{n}=\int_{\mathbb{R}} \frac{d \xi_{n}}{\left(1+\left|\xi_{n}\right|^{2}+\left|\xi^{\prime}\right|^{2}\right)^{s}}=\frac{1}{\left(1+\left|\xi^{\prime}\right|^{2}\right)^{s-\frac{1}{2}}} \int_{\mathbb{R}} \frac{d t}{\left(1+t^{2}\right)^{s}}
$$

\footnotetext{
${ }^{1}$ More generally, there is such a map from $H^{s}$ to $H^{s-\frac{1}{2}}$ for $s>\frac{1}{2}$ with all the other properties.

${ }^{2}$ More precisely, there is a continuous right inverse $\mathcal{E}: H^{\frac{1}{2}}\left(\partial M ;\left.E\right|_{\partial M}\right) \rightarrow H^{1}(M)$, which is the extension operator.
} 
and the last integral converges since $s>\frac{1}{2}$. By integrating A.1, we get:

$$
\left\|\left.v\right|_{\mathbb{R}^{n-1}}\right\|_{H^{s-\frac{1}{2}\left(\mathbb{R}^{n-1}\right)}} \leq C_{s}\|v\|_{H^{s}\left(\mathbb{R}^{n}\right)}
$$

for some constant $C_{s}$, which is what we wanted to prove. By density of $C^{\infty}$ sections we get the wanted inequality, i.e. $\left\|T_{1} u\right\|_{H^{s-\frac{1}{2}\left(\partial M ;\left.E\right|_{\partial M}\right)}} \leq C\|u\|_{H^{s}(M, E)}$ for a constant $C$ not depending on $u$ and a unique bounded extension.

For surjectivity of $T$ we omit the proof, as it is slightly more involved and refer the reader to Lemma 7.41 in [1], where it is proved using interpolation theory.

We follow the proof of Theorem 2 on page 273 of 30 to prove that $\operatorname{ker} T=H_{0}^{1}(M ; E)$, by taking the usual partition of unity and going to charts we reduce the problem to spaces on $\mathbb{H}^{n}=\left\{x_{n}>0\right\}$ and suppose $u \in H^{1}\left(\mathbb{H}^{n}\right)$ with compact support and $T u=0$. Approximate $u$ in $H^{1}$ by a sequence $u_{i}$ of smooth functions with compact support in $\overline{\mathbb{H}^{n}}$. Then

$$
\left|u_{m}\left(x^{\prime}, x_{n}\right)\right| \leq\left|u_{m}\left(x^{\prime}, 0\right)\right|+\int_{0}^{x_{n}}\left|\frac{\partial u_{m}}{\partial x_{n}}\right| d t
$$

for $x_{n} \geq 0$ and so by squaring, integrating, using Cauchy-Schwarz and taking $m \rightarrow \infty$ we get:

$$
\int_{\mathbb{R}^{n-1}}\left|u\left(x^{\prime}, x_{n}\right)\right|^{2} d x^{\prime} \leq C x_{n} \int_{0}^{x_{n}} \int_{\mathbb{R}^{n-1}}|D u|^{2} d x^{\prime} d t
$$

Now by introducing a function $\zeta \in C_{0}^{\infty}(\mathbb{R})$ with $\left.\zeta\right|_{[0,1]}=1$ and $\zeta_{m}(t)=\zeta(m t)$, we define $\omega_{m}:=u(x)\left(1-\zeta_{m}\right)$; by estimating and using A.2 we may prove that $D \omega_{i} \rightarrow D u$ in $L^{2}\left(\mathbb{H}^{n}\right)$ and so $\omega_{i} \rightarrow u$ in $H^{1}\left(\mathbb{H}^{n}\right)$. Therefore $u \in H_{0}^{1}\left(\mathbb{H}^{n}\right)$, as $\omega_{i} \in C_{0}^{\infty}\left(\mathbb{H}^{n}\right)$.

REMARK A.5. Note that now the Dirichlet problem (2.2) can be invariantly defined using $f$ in the intrinsic boundary Sobolev space $H^{\frac{1}{2}}\left(\partial M ;\left.E\right|_{\partial M}\right)$ - however, by Proposition A.4 these two set-ups are equivalent.

\section{A.2. The space of smooth curves and an extension lemma}

We need the metric space of smooth curves in the proof of our main theorem - here are some properties:

REMARK A.6. We are using the standard metric on the space $C^{\infty}([0,1] ; \mathbb{R})$ induced by the seminorms $\|f\|_{k}=\sup _{t \in[0,1]}\left|\frac{d^{k} f}{d t^{k}}\right|$. Then a choice of the metric on this space is:

$$
d(f, g)=\sum_{k=0}^{\infty} 2^{-k} \frac{\|f-g\|_{k}}{1+\|f-g\|_{k}}
$$

and it is a standard fact that this space is a Fréchet space with the same topology as the weak topology given by the seminorms. Furthermore, this also induces a Fréchet metric to the space $C^{\infty}\left([0,1] ; \mathbb{R}^{m}\right)=\oplus_{i=1}^{m} C^{\infty}([0,1] ; \mathbb{R})$ for all $m \in \mathbb{N}$. Moreover, we may consider the space $C^{\infty}([0,1] ; M)$ for any compact Riemannian manifold $(M, g)$ by isometrically embedding $M$ into a Euclidean space $\mathbb{R}^{N}$ for some $N$, as a closed subspace of $C^{\infty}\left([0,1], \mathbb{R}^{N}\right)$.

Now we prove the following lemma for the continuity of $h$ in the interior and on the boundary of the manifold.

Lemma A.7. Let $\Omega \subset \mathbb{R}^{n}$ be a domain and $E \subset \Omega$ a closed subset. Assume also that for any two points $x, y \in \Omega \backslash E$ and any smooth path $\gamma$ in $\Omega$ between $x$ and $y$, there exist smooth paths $\gamma_{i}$ from $x$ to $y$, lying in $\Omega \backslash E$, for $i=1,2, \ldots$, that converge to $\gamma$ in the metric space $C^{\infty}\left([0,1] ; \mathbb{R}^{n}\right)$. Let $f: \Omega \backslash E \rightarrow \mathbb{C}$ be a smooth function, such that $\partial^{\alpha} f$ extend continuously to $\Omega$ for all multi-indices $\alpha$. Then there exists a unique smooth extension $\tilde{f}: \Omega \rightarrow \mathbb{C}$ with $\left.\tilde{f}\right|_{\Omega \backslash E}=f$. 
Proof. This is a local claim, so we will consider an extension near a point $x \in E$. We will prove that the continuous extension $\tilde{f}$ of $f$ to $\Omega$ is differentiable with the derivative given by the continuous extension $h$ of $d f$ to $\Omega$. By inductively repeating the argument for all $\partial^{\alpha} f$ for multi-indices $\alpha$, it clearly suffices to prove this.

Consider the point $y=x+\delta e_{1}$, where $\delta>0$ is small enough so that the straight line path $\gamma$ between $x$ and $y$ lies in $\Omega$. Since $\Omega \backslash E$ is dense in $\Omega$, we may choose points $x^{\prime}, y^{\prime} \in \Omega \backslash E$ that are close to $x, y$, respectively. Consider the path $\gamma^{\prime}$ obtained by smoothing out the straight line path from $x^{\prime}$ to $x, \gamma$ and the straight line path from $y$ to $y^{\prime}$. By the hypothesis, there exists a sequence of paths $\gamma_{n}$ with endpoints at $x^{\prime}$ and $y^{\prime}$, lying entirely in $\Omega \backslash E$ that converge to $\gamma^{\prime}$ in the path metric.

We will consider the integrals along the curves $\gamma_{n}$ : after possibly reparametrising, we may assume that $\gamma_{n}$ are parametrised by arc-length - we can always do this for $n$ sufficiently large, as $\gamma$ has a nowhere zero derivative. Therefore, we may integrate $h\left(\dot{\gamma}_{n}\right)$ to get that, by the Fundamental Theorem of Calculus

$$
f\left(y^{\prime}\right)-f\left(x^{\prime}\right)=\int_{\gamma_{n}} d\left(f \circ \gamma_{n}(t)\right)=\int_{\gamma_{n}} h\left(\dot{\gamma}_{n}\right)
$$

Here, we think of $h$ as given by the vector of partial derivatives of $f$. By uniform convergence of the curves, we immediately get that

$$
f\left(y^{\prime}\right)-f\left(x^{\prime}\right)=\int_{\gamma_{n}} h\left(\dot{\gamma}_{n}\right) \rightarrow \int_{\gamma^{\prime}} h\left(\dot{\gamma}^{\prime}\right)
$$

and therefore, if we take $x^{\prime} \rightarrow x$ and $y^{\prime} \rightarrow y$ (we can do this as $\Omega \backslash E$ is dense in $\Omega$ ), we get:

$$
\frac{\tilde{f}\left(x+\delta e_{1}\right)-\tilde{f}(x)}{\delta}=\frac{1}{\delta} \int_{0}^{\delta} h_{x+t e_{1}}\left(e_{1}\right) d t \rightarrow h_{x}\left(e_{1}\right)
$$

as $\delta \rightarrow 0$. Therefore, the partial derivative in the $e_{1}$ direction exists and similarly, all other partials exist and are equal to the components of $h$. This finishes the proof.

REMARK A.8. If we are given a smooth function $f$ in the interior of a domain $\Omega \subset \mathbb{R}^{n}$ with smooth boundary, such that all derivatives $\partial^{\alpha} f$ extend continuously to the boundary, it is well known that there exists a smooth extension $\tilde{f}$ to $\mathbb{R}^{n}$, such that $\left.\tilde{f}\right|_{\Omega}=f$. This remark, together with the above lemma, are used in the proof of the smooth extension of $h$ over the singular set in Theorem G.

Finally, we would like to recall the well-posedness conditions under which the solution operator to a generalised heat equation is smoothing. One set of such conditions is given by (1.5)-(1.7) on page 134 in Treves $[\mathbf{8 0}]$ - we state them here for completeness. Let $X$ be a manifold of dimension $n$ and $t$ a variable in the real line $\mathbb{R}$; we will consider vector functions with values in the finite dimensional space $H=\mathbb{C}^{m}$. Let $A(t)$ be a pseudodifferential operator of order $k$ with values in $L(H)=\mathbb{C}^{m \times m}$ depending smoothly on $t \in[0, T)$; this means that in a local chart $\Omega \subset X$ we have the symbol of $A(t)$ modulo $S^{-\infty}$ being a smooth function $a_{\Omega}(x, t, \xi):[0, T) \rightarrow S^{k}(\Omega ; L(H))$. We consider the following equation in $X \times[0, T)$, where $U$ valued in $H$ :

$$
\frac{d U}{d t}-A(t) \circ U \equiv 0 \quad \text { modulo } S^{-\infty}
$$

The set of conditions for this equation to be well-posed is the following:

Condition A.9 (Well-posedness of the heat equation). For every local chart $\Omega \subset X$, there is a symbol $a(x, t, \xi)$ depending smoothly on $t \in[0, T)$ and defining a pseudodifferential operator $A_{\Omega}(t)$ congruent to $A(t)$ modulo regularising operators in $\Omega$, such that for every compact $K \subset$ 
$\Omega \times[0, T)$ there is a compact subset $K^{\prime}$ of the open half-plane $\mathbb{C}_{-}=\{z \in \mathbb{C} \mid \operatorname{Re}(z)<0\}$ such that

$$
z \times I d-\frac{a(x, t, \xi)}{\left(1+|\xi|^{2}\right)^{\frac{m}{2}}}: H \rightarrow H
$$

is a bijection for all $(x, t) \in K, \xi \in \mathbb{R}^{n}$ and $z \in \mathbb{C} \backslash K^{\prime}$.

One remark is in place after this condition:

REMARK A.10. In fact, the symbol of the Laplace operator in the ordinary heat equation does not immediately satisfy Condition A.9 for a well-posed (generalised) heat equation - if one plugs $-|\xi|^{2}(m=1)$ into A.3 , we have that the zero set spreads such that we have $\operatorname{Re}(z) \in(-1,0)$ and $\operatorname{Im}(z)=0$, which is certainly not contained in a compact subset of $\mathbb{C}_{-}=\{\operatorname{Re}(z)<0\}$; the trick is to add a factor of $e^{-|\xi|^{2}}$ which does not change the class of the symbol modulo $S^{-\infty}$, as we will see in the proof of the Lemma below.

Using the idea in the above remark, we prove that the operator we use in Proposition 3.3 satisfies Condition A.9.

Lemma A.11. The $\mathbb{C}^{m \times m}$-valued pseudodifferential operator $A=B-E \times I d$ (defined in Lemma 3.2) satisfies Condition A.9.

Proof. Denote by $a_{1}=-\sqrt{q_{2}}=-\sqrt{\sum_{\alpha, \beta} g^{\alpha \beta} \xi_{\alpha} \xi_{\beta}}$ the principal symbol of $A$ ( $E$ has degree zero). If $K \subset[0, T] \times \mathbb{R}^{n-1}$ compact, then there exist positive $C_{1}, C_{2}$ and $c$ such that

$$
\begin{aligned}
c|\xi| \leq\left|a_{1}(x, t, \xi)\right| & \leq C_{1}\left(1+|\xi|^{2}\right)^{\frac{1}{2}} \\
\left|a_{0}(x, t, \xi)\right| & \leq C_{2}
\end{aligned}
$$

for all $(x, t) \in K$ and $\xi \in \mathbb{R}^{n-1}$, by definition of symbols and the fact that $g^{\alpha \beta}$ is positive definite. Thus we can rewrite:

$$
z \times I d-\frac{-\sqrt{q_{2}} \times I d+a_{0}}{\left(1+|\xi|^{2}\right)^{\frac{1}{2}}}=\left(z+\frac{\sqrt{q_{2}}}{\left(1+|\xi|^{2}\right)^{\frac{1}{2}}}\right) \times I d-\frac{a_{0}}{\left(1+|\xi|^{2}\right)^{\frac{1}{2}}}
$$

and if this expression is singular, we ought to have

$$
\frac{\left|a_{0}\right|^{2}}{1+|\xi|^{2}} \geq m^{2}\left|z+\frac{\sqrt{q_{2}}}{\left(1+|\xi|^{2}\right)^{\frac{1}{2}}}\right|^{2}=m^{2}|s|^{2}+m^{2}\left(r+\frac{\sqrt{q_{2}}}{\left(1+|\xi|^{2}\right)^{\frac{1}{2}}}\right)^{2}
$$

where $z=r+i s$. If we had $|\xi|$ large enough and $r \geq-\epsilon$ for some small $\epsilon>0$, the left hand side of A.5 would be small and the right hand side of it would be bigger than $s^{2}+\left(r+\frac{c}{2}\right)^{2}$ (up to a constant). Therefore for $|\xi| \geq K$ for some $K$, A.4 will be non-singular for $r \geq-\epsilon$.

Notice that in the condition we have the freedom of adding a smoothing factor - this will take care of the singular behaviour for $|\xi|$ in a compact set. We will add a factor of $C e^{-|\xi|^{2}} \times I d \in S^{-\infty}$ for some $C>0$ to remedy this. First of all, notice that the above argument remains the same with the same $|\xi|$, if we consider the symbol $\sqrt{q_{2}} \times I d+a_{0}+C e^{-|\xi|^{2}} \times I d$.

Furthermore, we have the left hand side of (A.5) bounded for all $\xi$ uniformly, whereas the right hand side is bigger (up to a constant) than $\left(C e^{-|\xi|^{2}}-\epsilon\right)^{2}$ for $r \geq-\epsilon$, large enough $C$ and $|\xi| \leq K$. Clearly this inequality fails to hold for large $C$ and this finishes the proof. 


\section{Bibliography}

[1] R. A. Adams, J. J. F. Fournier, Sobolev spaces, Second edition, Pure and Applied Mathematics (Amsterdam) 140, Elsevier/Academic Press, Amsterdam (2003).

[2] P. Albin, C. Guillarmou, L. Tzou, G. Uhlmann, Inverse boundary problems for systems in two dimensions, Ann. Henri Poincaré 14 (2013), 1551-1571.

[3] J. Anderson, L. Tzou, Stability for a magnetic Schrödinger operator on a Riemann surface with boundary, Inverse Problems (2017, submitted), 1-30.

[4] M. F. Atiyah, R. Bott, The Yang-Mills equations over Riemann surfaces, Philos. Trans. Roy. Soc. London Ser. A 308 (1983), no. 1505, 523-615.

[5] T. Aubin, Some nonlinear problems in Riemannian geometry, Springer Monographs in Mathematics, Springer-Verlag, Berlin, 1998.

[6] E. P. van den Ban, M. Crainic, Lecture notes on Analysis on Manifolds: online source (2009).

[7] C. Bär, Zero sets of solutions to semilinear elliptic systems of first order, Invent. Math. 138 (1999), 183-202.

[8] B. Booss-Bavnbek, K. P. Wojciechowski, Elliptic boundary problems for Dirac operators. Mathematics: Theory \& Applications, Birkhäuser Boston, Inc., Boston, MA, 1993. xviii+307 pp.

[9] R. M. Brown, M. Salo, Identifiability at the boundary for first-order terms, Appl. Anal. 85 (2006), $735-749$.

[10] G. Uhlmann, Developments in inverse problems since Calderóns foundational paper, Harmonic analysis and partial differential equations (Chicago, IL, 1996), 295-345, Chicago Lectures in Math., Univ. Chicago Press, Chicago, IL (1999).

[11] M. Cekić, The Calderón problem for connections, preprint (2016), arXiv: 1610.02985.

[12] M. Cekić, A contribution to the Calderón problem for Yang-Mills connections, preprint (2017), arXiv: 1704.01362 .

[13] F. J. Chung, A partial data result for the magnetic Schrödinger inverse problem, Anal. PDE 7 (2014), 117-157.

[14] F. J. Chung, M. Salo, L. Tzou, Partial data inverse problems for the Hodge Laplacian, Anal. PDE (to appear), arXiv:1310.4616.

[15] K. Datchev, H. Hezari, Inverse problems in spectral geometry, Inverse problems and applications: inside out. II, 455-485, Math. Sci. Res. Inst. Publ. 60, Cambridge Univ. Press, Cambridge, 2013.

[16] L. De Carli, S. M. Hudson, Geometric remarks on the level curves of harmonic functions (English summary), Bull. Lond. Math. Soc. 42 (2010), no. 1, 83-95.

[17] M. Dimassi, J. Sjstrand, Spectral asymptotics in the semi-classical limit (English summary), London Mathematical Society Lecture Note Series 268, Cambridge University Press, Cambridge (1999), xii+227 pp.

[18] S. K. Donaldson, P. B. Kronheimer, The geometry of four-manifolds, Oxford Mathematical Monographs, The Clarendon Press, Oxford University Press, New York, 1990.

[19] S. K. Donaldson, Mathematical uses of gauge theory, in: The Encyclopedia of Mathematical Physics, Elsevier (2006).

[20] D. Dos Santos Ferreira, Microlocal analysis and inverse problems, lecture notes (2011), available online

[21] D. Dos Santos Ferreira, C. Kenig, M. Salo, G. Uhlmann, Limiting Carleman weights and anisotropic inverse problems, Invent. Math. 178 (2009), 119-171. 
[22] D. Dos Santos Ferreira, C. Kenig, J. Sjöstrand, G. Uhlmann, Determining a magnetic Schrödinger operator from partial Cauchy data, Comm. Math. Phys. 271 (2007), 467-488.

[23] D. Dos Santos Ferreira, Y. Kurylev, M. Lassas, M. Salo, The Calderón problem in transversally anisotropic geometries, J. Eur. Math. Soc. (to appear), arXiv:1305.1273.

[24] S. Dyatlov, M. Zworski, Mathematical theory of scattering resonances (Appendix E), in preparation (2017): online source.

[25] M. Eller, V. Isakov, G. Nakamura, D. Tataru, Uniqueness and stability in the Cauchy problem for Maxwell and elasticity systems, in: Nonlinear partial differential equations and their applications, Collège de France Seminar, Vol. XIV, in: Stud. Math. Appl. 31, North-Holland, Amsterdam, 2002, pp. 329-349.

[26] G. Eskin, Global uniqueness in the inverse scattering problem for the Schrdinger operator with external Yang-Mills potentials, Comm. Math. Phys. 222 (2001), 503-531.

[27] G. Eskin, J. Ralston, Inverse scattering problem for the Schrödinger equation with magnetic potential at a fixed energy, Comm. Math. Phys. 173 (1995), 199-224.

[28] G. Eskin, J. Ralston, Inverse scattering problems for Schrödinger operators with magnetic and electric potentials, Inverse problems in wave propagation (Guy Chavent et al., ed.), IMA Volumes in Mathematics and Applications, no. 90 (1997), pp. 147-166.

[29] G. Eskin, J. Ralston, On the inverse boundary value problem for linear isotropic elasticity and Cauchy-Riemann systems, in Inverse problems and spectral theory, Contemp. Math. 348, Amer. Math. Soc., Providence, RI, 2004, pp. 53-69.

[30] L. Evans, Partial differential equations, Graduate Studies in Mathematics 19, American Mathematical Society, Providence, RI (1998).

[31] F. G. Friedlander, Introduction to the theory of distributions, Second edition, With additional material by M. Joshi, Cambridge University Press, Cambridge, 1998. x+175 pp.

[32] O. Forster, Lectures on Riemann surfaces, Graduate Texts in Mathematics 81, Springer-Verlag, New York, 1991.

[33] C. Guillarmou, Lens rigidity for manifolds with hyperbolic trapped set, J. Amer. Math. Soc. (to appear), arXiv:1412.1760.

[34] C. Guillarmou, G. Paternain, M. Salo, G. Uhlmann, The X-ray transform for connections in negative curvature, Comm. Math. Phys. 343 (2016), 83-127.

[35] C. Guillarmou, A. Sá Barreto, Inverse problems for Einstein manifolds, Inverse Probl. Imaging 3 (2009), 1-15.

[36] C. Guillarmou, L. Tzou, Identification of a connection from Cauchy data on a Riemann surface with boundary, Geom. Funct. Anal. 21 (2011), 393-418.

[37] B. Haberman, Unique Determination of a Magnetic Schrdinger Operator with Unbounded Magnetic Potential from Boundary Data, International Mathematics Research Notices (2016), 1-49.

[38] M. W. Hirsch, Differential topology, Graduate Texts in Mathematics 33, Springer-Verlag, New York, 1994.

[39] C. E. Kenig, M. Salo, G. Uhlmann, Inverse problems for the anisotropic Maxwell equations, Duke Math. J. 157 (2011), 369-419.

[40] C. E. Kenig, J. Sjöstrand, G. Uhlmann, The Calderón problem with partial data, Ann. of Math. (2) 165 (2007), 567-591.

[41] K. Knudsen, M. Salo, Determining nonsmooth first order terms from partial boundary measurements, Inverse Probl. Imaging 1 (2007), 349-369.

[42] S. Kobayashi, Differential geometry of complex vector bundles, Publications of the Mathematical Society of Japan 15, Princeton University Press, Princeton, NJ; Iwanami Shoten, Tokyo, 1987.

[43] S. Kobayashi, K. Nomizu, Foundations of differential geometry, Vol I. Interscience Publishers, a division of John Wiley \& Sons, New York-London (1963) xi+329 pp.

[44] Y. Kurylev, L. Oksanen, G. P. Paternatin, Inverse problems for the connection Laplacian, arxiv preprint (2015). 
[45] K. Krupchyk, G. Uhlmann, Uniqueness in an inverse boundary problem for a magnetic Schrödinger operator with a bounded magnetic potential, Comm. Math. Phys. 327 (2014), no. 3, 993-1009.

[46] K. Krupchyk, G. Uhlmann, Inverse problems for magnetic Schrödinger operators in transversally anisotropic geometries, preprint (2017), arXiv: 1702.07974.

[47] M. Lassas, T. Liimatainen, M. Salo, The Calderón problem for the conformal Laplacian, arxiv preprint (2016).

[48] M. Lassas, M. Taylor, G. Uhlmann, The Dirichlet-to-Neumann map for complete Riemannian manifolds with boundary, Comm. Anal. Geom. 11 (2003), no. 2, 207-221.

[49] M. Lassas, G. Uhlmann, On determining a Riemannian manifold from the Dirichlet-to-Neumann map, Ann. Sci. cole Norm. Sup. (4) 34 (2001), 771-787.

[50] J. M. Lee, G. Uhlmann, Determining anisotropic real-analytic conductivities by boundary measurements, Comm. Pure Appl. Math. 42 (1989), no. 8, 1097-1112.

[51] T. Liimatainen, M. Salo, Nowhere conformally homogeneous manifolds and limiting Carleman weights, Inverse Probl. Imaging 6 (2012), no. 3, 523-530.

[52] A. Martinez, An introduction to semiclassical and microlocal analysis, Universitext, SpringerVerlag, New York (2002), viii+190 pp.

[53] R. Mazzeo, Unique continuation at infinity and embedded eigenvalues for asymptotically hyperbolic manifolds, Amer. J. Math. 113 (1991), 25-45.

[54] R. Melrose, Lecture notes on Microlocal Analysis: online source (2003).

[55] H. B. Jr. Lawson, M-L. Michelsohn, Spin geometry, Princeton Mathematical Series, 38. Princeton University Press, Princeton, NJ (1989). xii+427 pp.

[56] C. B. Morrey Jr., On the analyticity of the solutions of analytic non-linear elliptic systems of partial differential equations. I. Analyticity in the interior, Amer. J. Math. 80 (1958), 198-218.

[57] G. Nakamura, G. Uhlmann, Complex geometrical optics solutions and pseudoanalytic matrices, Ill-posed and inverse problems, 305-338, VSP, Zeist, 2002.

[58] G. Nakamura, G. Uhlmann, Global uniqueness for an inverse boundary value problem arising in elasticity, Invent. Math. 118 (1994), 457-474.

[59] G. Nakamura, G. Uhlmann, Erratum: Global uniqueness for an inverse boundary value problem arising in elasticity, Invent. Math. 152 (2003), 205207.

[60] G. Nakamura, Z. Sun, Z, G. Uhlmann, Global identifiability for an inverse problem for the Schrödinger equation in a magnetic field, Math. Ann. 303 (1995), no. 3, 377-388.

[61] G. Nakamura, G. Uhlmann, A layer stripping algorithm in elastic impedance tomography, IMA Vol. Math. Appl. 90, Springer, New York (1997), 375-384.

[62] R.G. Novikov, G.M. Khenkin, The $\bar{\partial}$-equation in the multidimensional inverse scattering problem, Russ. Math. Surv. 42 (1987), 109-180.

[63] A. Panchenko, An inverse problem for the magnetic Schrödinger equation and quasi-exponential solutions of nonsmooth partial differential equations, Inverse Problems 18 (2002), 1421-1434.

[64] G. P. Paternain, Inverse problems for connections, Inverse problems and applications: inside out II, 369-409, Math. Sci. Res. Inst. Publ. 60, Cambridge Univ. Press, Cambridge, 2013.

[65] G. P. Paternain, M. Salo, G. Uhlmann, Tensor tomography: progress and challenges, Chinese Annals of Math. Ser. B 35 (2014), 399-428.

[66] G. P. Paternain, M. Salo, G. Uhlmann, H. Zhou, The geodesic X-ray transform with matrix weights, preprint (2016), arXiv:1605.07894.

[67] L. Pestov, G. Uhlmann, Two dimensional compact simple Riemannian manifolds are boundary distance rigid, Ann. of Math. (2) 161 (2005), 1093-1110.

[68] J. Radon, Über die Bestimmung von Funktionen durch ihre Integralwerte langs gewisser Mannigfaltigkeiten, Ber. Verh. Sach. Akad. 69 (1917), 262-277.

[69] W. Rudin, Functional analysis, Second edition, International Series in Pure and Applied Mathematics, McGraw-Hill, Inc., New York, 1991. xviii+424 pp. 
[70] M. Salo, The Calderón problem on Riemannian manifolds, Inverse problems and applications: inside out. II, 167-247, Math. Sci. Res. Inst. Publ. 60, Cambridge Univ. Press, Cambridge, 2013.

[71] M. Salo, Inverse boundary value problems for the magnetic Schrödinger equation, J. Phys. Conf. Series 73 (2007), 012020.

[72] M. Salo, Inverse problems for nonsmooth first order perturbations of the Laplacian, Ann. Acad. Sci. Fenn. Math. Diss. 139 (2004), 67 pp.

[73] M. A. Shubin, Pseudodifferential operators and spectral theory (Translated from the 1978 Russian original by Stig I. Andersson), Second edition, Springer-Verlag, Berlin, 2001. xii+288 pp.

[74] P. Stefanov, G. Uhlmann, A. Vasy, Inverting the local geodesic X-ray transform on tensors, J. Anal. Math. (to appear), arXiv:1410.5145.

[75] P. Stefanov, G. Uhlmann, A. Vasy, Local and global boundary rigidity and the geodesic X-ray transform in the normal gauge, preprint (2017), arXiv:1702.03638.

[76] R. S. Strichartz, Analysis of the Laplacian on the complete Riemannian manifold, J. Funct. Anal. 52 (1983), 48-79.

[77] Z. Sun, An inverse boundary value problem for Schrödinger operators with vector potentials, Trans. Amer. Math. Soc. 338 (1993), no. 2, 953-969.

[78] J. Sylvester, G. Uhlmann, A global uniqueness theorem for an inverse boundary value problem, Ann. of Math. (2) 125 (1987), 153-169.

[79] C. Tolmasky, Exponentially growing solutions for nonsmooth first-order perturbations of the Laplacian, SIAM J. Math. Anal. 29 (1998), 116-133.

[80] F. Treves, Introduction to Pseudodifferential Operators and Fourier Integral Operators, Plenum Press, New York, 1980.

[81] L. Tzou, Stability estimates for coefficients of magnetic Schrdinger equation from full and partial boundary measurements, Comm. Partial Differential Equations 33 (2008), 1911-1952.

[82] L. Tzou, The reflection principle and Calderón problems with partial data, Mathematische Annalen (2017), 3-46.

[83] K. K. Uhlenbeck, Connections with Lp bounds on curvature, Comm. Math. Phys. 83 (1982), 31-42.

[84] G. Uhlmann, Electrical impedance tomography and Calderón's problem, Inverse Problems 25 (2009), 39 pp.

[85] G. Uhlmann, A. Vasy, The inverse problem for the local geodesic ray transform (with an appendix by H. Zhou), Invent. Math. 205 (2016), 83-120.

[86] R. A. Walker, Problems in Harmonic Function Theory, Honors Theses (1998), Paper 492.

[87] M. Zworski, Semiclassical analysis, Graduate Studies in Mathematics, 138, American Mathematical Society, Providence, RI (2012). xii+431 